\title{
Synthesis of Multi-walled Carbon Nanotubes by Chemical Vapour Deposition and Post-synthesis Treatment by Dielectrophoresis
}

By

Xianming Liu

\author{
A thesis \\ submitted to the Victoria University of Wellington \\ in fulfillment of the \\ requirement for the degree of \\ Doctor of Philosophy in Chemistry
}

Victoria University of Wellington

2006 


\begin{abstract}
Carbon nanotubes (CNTs) are a group of pure carbon solid materials that possess onedimensional structures with diameters down to less than one nanometre. They have interesting physical properties such as very high tensile strength, metallic and semiconducting conductivity, and great potential for applications.

This work investigates the synthesis, alignment and purification of multi-walled carbon nanotubes, which were characterized by Electron Microscopy, measurement of electrical properties and Raman scattering.

Synthesis of multi-walled carbon nanotubes (MWNTs) was carried out by Chemical Vapour Deposition, using three different precursors: $\mathrm{Fe}\left(\mathrm{NO}_{3}\right)_{3}$, ferrocene and iron phthalocyanine. Vertically aligned "forests" of large numbers of MWNTs were achieved using ferrocene and iron phthalocyanine as precursors. Products from iron phthalocyanine yield more graphitic CNT nanostructures, as determined by Scanning Electron Microscopy, Transmission Electron Microscopy, Thermogravity Analysis, Raman microscopy and Energy Dispersive X-ray spectroscopy. Patterned growth of vertical MWNTs arrays with a resolution of 2 microns was also obtained, using a predeposited substrate. A High Resolution Transmission Electron Microscope was employed to investigate the inner structures of individual MWNTs, giving well-resolved images of concentric nanostructures with inter-lattice spacings of $0.34 \mathrm{~nm}$.
\end{abstract}


Techniques for purification and manipulation of CNTs are required before CNTs' excellent properties can be exploited. In this thesis, dielectrophoresis (DEP) under highfrequency $\mathrm{AC}$ voltages using an array of micron-scale electrodes was adopted as the technique to align MWNTs between these electrodes. This technique is effective for MWNTs since their long cylindrical structure and the high mobility of their charge carriers allow them to be electrically polarised. As a result, MWNTs experience large $\mathrm{DEP}$ forces in an $\mathrm{AC}$ electric field. In our experiments, inter-digitated electrodes were used to apply the electric field to CNT suspensions in various solvents. Alignment of CNTs along their axis was achieved within the gaps between adjacent electrodes at a frequency larger than $1.1 \mathrm{MHz}$. The $\mathrm{AC}$ admittance changes between the electrodes were monitored and were observed to be a clear reflection of the accumulation process of MWNTs. Also it was identified that distilled water was a better solvent than ethanol and isopropanol for DEP purpose on MWNTs.

In addition, a prototype device was built to selectively purify MWNTs from as-grown samples by combining DEP and re-circulating fluid flow. It was found that this device is able to decrease the impurity content of MWNTs, which is collected on the electrode array, from the suspension of as-grown samples. 


\section{Acknowledgement}

I would like to express my sincere gratitude to all the people who have been helping me with this thesis. First of all, I would like to thank Prof. John Spencer and Prof. Alan Kaiser, who supervised my research and thesis, from the bottom of my heart. I benefit so much from their profound academic knowledge, philosophy and their care about my study, life and career. The last several years I spent with them on my study and this thesis is such a valuable experience to me.

Then I would like to thank

Dr. Mike Arnold and Dr. Ashton Partridge for instructing me on my research work in Industrial Research Limited,

Dr. Chris Bumby for proofreading my draft and the discussions on the introduction, DEP models and my experimental results in this thesis,

Dr. Richard Tilley and Mr. David Flynn for their helps on HRSEM and HRTEM, and Prof. Joe Trodahl and Dr. Pablo Etchegoin for their helps and instructions on Raman spectroscopy,

and my fellow students, Kirsten Edgar, Ray Maclachlan and Ray Vagana for their discussions and friendship.

And finally I would like to thank my family for supporting me during my $\mathrm{PhD}$, particularly my wife, Xiaonan for her deep love and encouraging me to complete my $\mathrm{PhD}$ study in Wellington. 


\section{Contents}

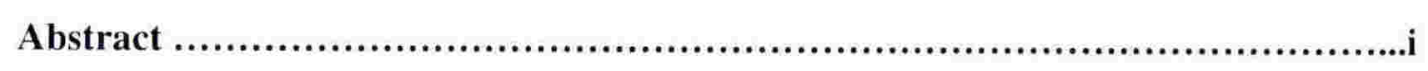

Acknowledgements................................................................

List of Figures.................................................................ii

List of Tables...................................................................

1 Introduction to Carbon Nanotubes..........................................

1.1 Carbon-based solid materials .........................................

1.1.1 Carbon and hybridisation...............................................

1.1.2 Electronic structure of graphite........................................

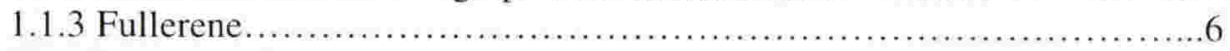

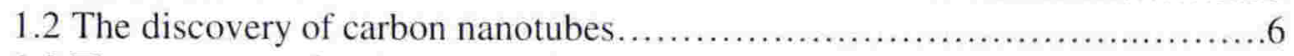

1.3 The structure of carbon nanotubes..................................... 7

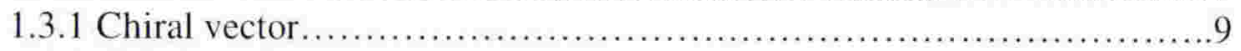

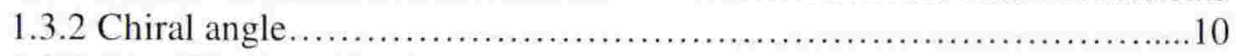

1.3.3 Classification of carbon nanotube...................................... 11

1.4 Electronic properties of carbon nanotubes................................ 11

1.4.1 Metallic carbon nanotubes as quantum wires........................ 14

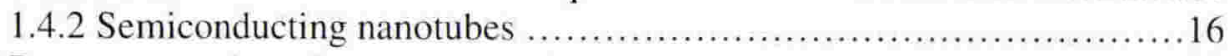

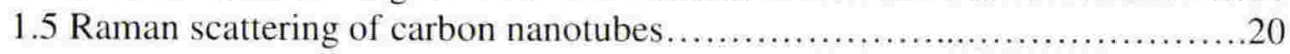

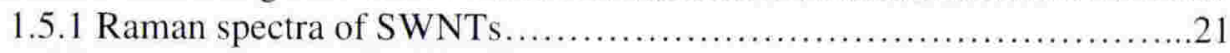

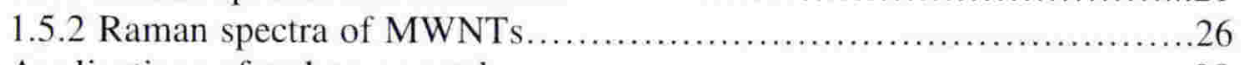

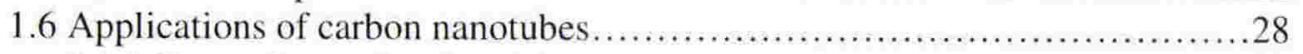

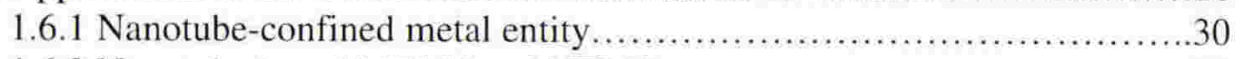

1.6.2 Nanotube-based MEMS and NEMS .................................30

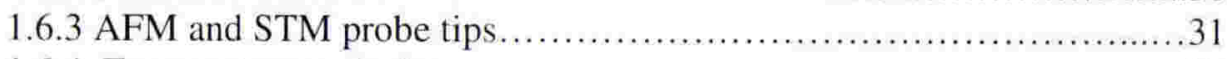

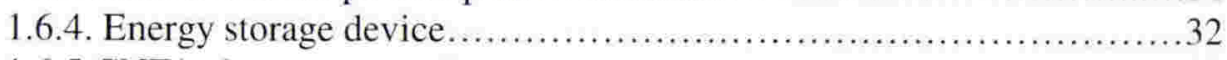

1.6.5 CNT/polymer composites.....................................32 


\section{PART I SYNTHESIS OF CARBON NANOTUBES BY CHEMICAL VAPOUR DEPOSITION}

2 Synthesis of Carbon Nanotubes.............................................43

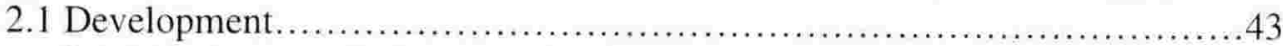

2.1.1 Carbon arc discharge method .....................................43

2.1.2 Laser ablation method .......................................... 45

2.1.3 Chemical vapour deposition........................................45

2.2 Chemical Vapour Deposition.............................................46

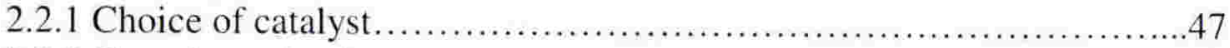

2.2.2 Growth mechanism............................................. 48

2.2.3 Role of hydrogen in the reaction..................................

2.3 Alignment of carbon nanotubes.......................................53

2.4 Micro-patterning of carbon nanotubes....................................55

3 Synthesis and Characterization of Multi-walled Carbon Nanotubes by

Chemical Vapour Deposition...............................................61

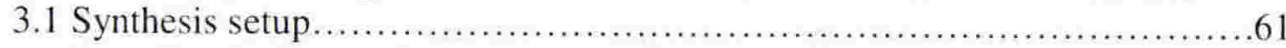

3.1.1 Synthesis of carbon nanotubes from $\mathrm{Fe}\left(\mathrm{NO}_{3}\right)_{3}$-derived nanoparticles...62

3.1.2 Synthesis of aligned MWCNTs by CVD .............................6. 63

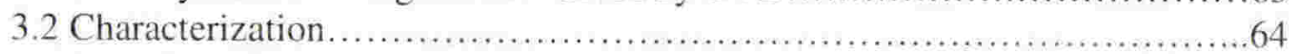

3.2.1 Scanning Electron Microscopy (SEM) ............................64

3.2.2 Transmission Electron Microscopy (TEM) ............................65

3.2.3 High Resolution Transmission Electron Microscopy ......................66

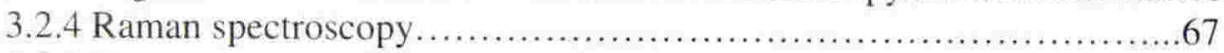

3.2.5 Thermogravimetric loss analysis (TGA) ...........................67

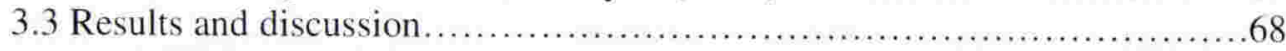

3.3.1 SEM and TEM investigations on carbon nanotubes from $\mathrm{Fe}\left(\mathrm{NO}_{3}\right)_{3^{-}}$

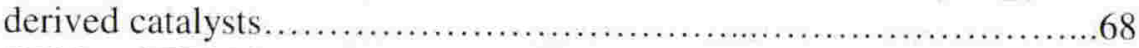

3.3.2 SEM and TEM investigations on aligned carbon nanotubes.............70

3.3.3 EDX characterization of carbon nanotubes produced from ferrocene-derived and FePc-derived catalysts........................ 80

3.3.4 Investigation on nanostructures inside individual MWNTs by High Resolution TEM.............................................. 83

3.3.5 TGA investigation of carbon nanotubes produced from ferrocenederived and FePc-derived catalysts.................................. 86

3.3.6 Raman spectroscopy on carbon nanotubes........................88

3.3.7 Growth mechanism of aligned carbon nanotubes..................... 93 


\section{POST-SYNTHESIS TREATMENT ON CARBON NANOTUBES BY} DIELECTROPHORESIS

4 Post-synthesis Treatment of Carbon Nanotubes.............................99

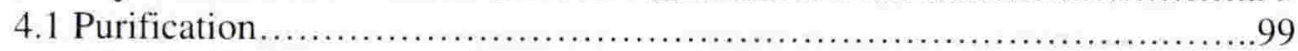

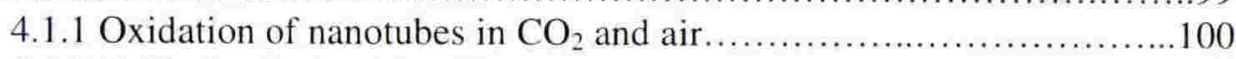

4.1 .2 Oxidation by liquid oxidants......................................... 100

4.1 .3 Ultrasonically assisted filtration.................................. 101

4.1.4 Reflux and extraction by organic solvents.............................. 101

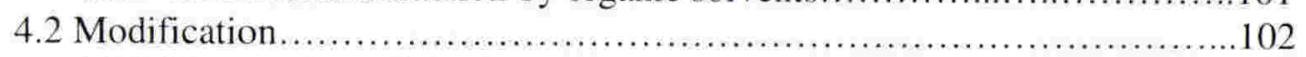

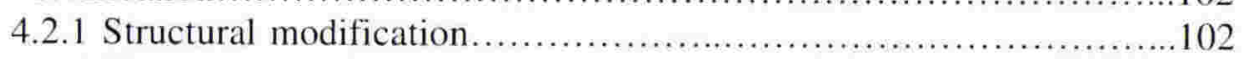

4.2.2 Chemical modification................................................ 103

4.3 Separating semiconducting and metallic nanotubes............................ 106

4.3.1 Selective physisorption............................................. 106

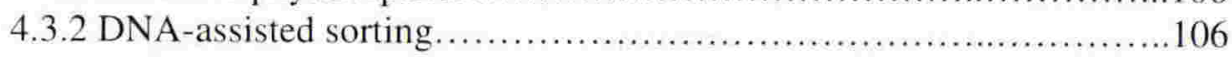

4.4 Post-synthesis alignments and positioning................................ 107

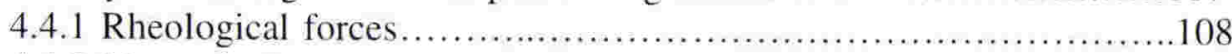

4.4.2 Magnetic force ................................................. 108

4.4 .3 Self assembly ............................................... 109

$5 \quad$ Dielectrophoresis............................................................ 114

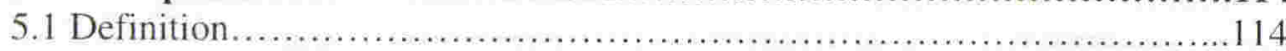

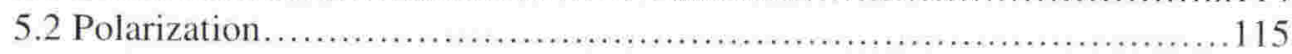

5.3 Dielectrophoretic force...................................................... 116

5.4 Dielectrophoresis and carbon nanotubes.................................. 118

5.4.1 Theoretical background ..................................... 118

5.4.2 Applications of dielectrophoresis for post-synthesis treatment of nanotubes................................................... 120

$6 \quad$ Alignment of Carbon Nanotubes by Dielectrophoresis......................127

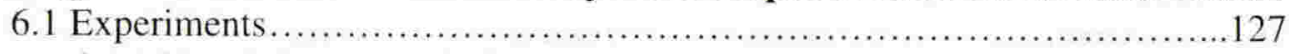

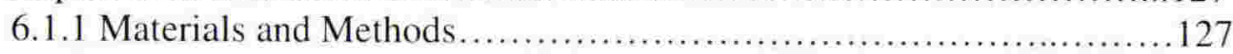

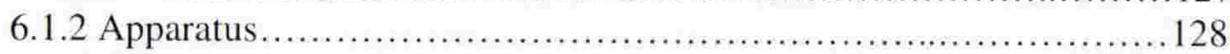

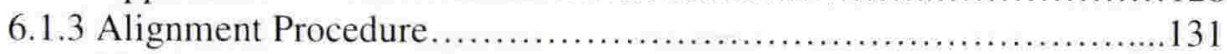

6.1.4 Simultaneous Dielectric Spectroscopy.................................131

6.1.5 Response of accumulated CNTs to on/off switching of the electric

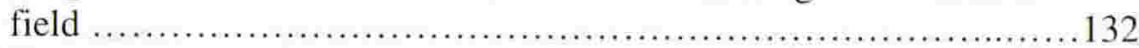

6.1.6 Frequency dependence investigation............................... 133

6.1.7 A 4-electrode structure........................................... 133

6.2 Results and Discussion............................................ 134

6.2.1 Optical microscopy images and dielectric spectrometry...............135

6.2.2 Further optical microscopy observations and SEM investigation.......138

6.2.3 Comparison of the dielectrophoretic behaviour between two types of CNTs. 


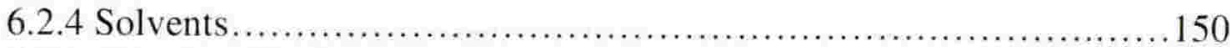

6.2.5 Aligning Single-walled Carbon Nanotubes with a 4-electrode Structure

7 Selective Purification of Multi-walled Carbon Nanotubes by Dielectrophoresis within a Large Array ...................................154

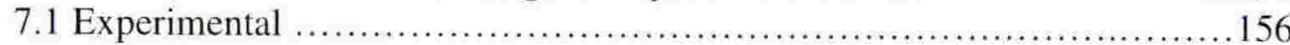

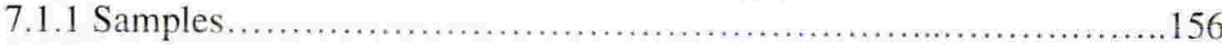

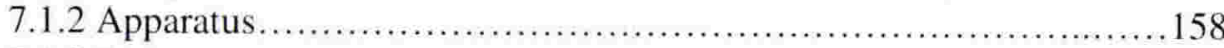

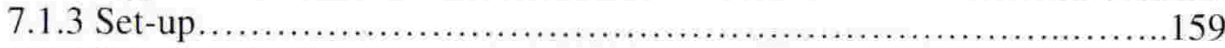

7.1.4 Characterisation................................................... 160

7.2 Results and Discussion................................................. 160

7.2.1 Optical microscopic and SEM images................................. 161

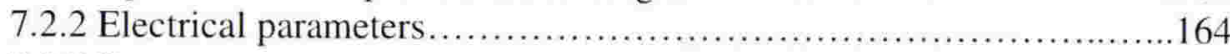

7.2.3 Raman spectra................................................ 165

7.2.4 Uniformity of collections...................................... 167

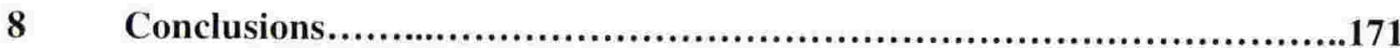

8.1 Synthesis of multi-walled carbon nanotubes by chemical vapour deposition........................................................ 171

8.2 Application of dielectrophoresis for aligning and selecting of carbon nanotubes............................................................ 173

8.3 A prototype device for separating MWNTs and impurities..................... 175

8.4 Electro-spinning of Poly Ethylene Oxide (PEO) fibres......................176

Appendix Electro-spinning of PEO Fibres...................................177 


\section{List of Figures}

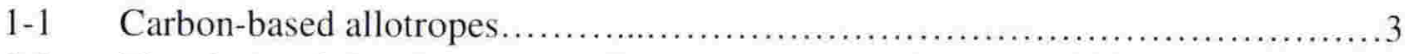

1-2 The electronic band structure of a graphite sheet calculated within a $\pi$-band tightbinding model. The valence $\pi$ and conduction bands $\pi^{*}$ show degeneracy at the $\mathrm{K}$ points in the Brillouin zone at the Fermi level.................................5

1-3 Schematic cross-sections of (a) a single-walled CNT with ten carbon atoms around the circumference and (b) a 4-layered multi-walled CNT. Unrolled lattice of a carbon nanotube .......................................................... 8

1-4 Unrolled lattice of a carbon nanotube ........................................ 9

1-5 Classification of single-walled carbon nanotubes ............................11

1-6 Electronic band structure versus wave vector of singwall nanotubes with chiral indices of $(5,5),(9,0)$ and $(10,0)$ derived from the band structure of graphene...13

1-7 (a) Ballistic transport through a multi-walled nanotube. (b) A plot of conductance versus depth of the nanotube section in the mercury at the room temperature ....16

1-8 (a) An AFM image of an individual carbon nanotube on top of three Pt electrodes. (b) Schematic side view of the nanotube-based field effect transistor. (c) I - V characteristic of the nanotube-based FET at different

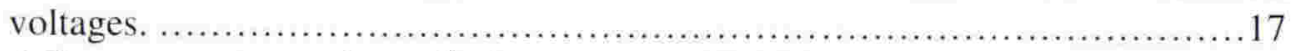

1-9 A Raman spectrum of arc discharge-grown SWNTs ..........................22

1-10 A Kataura plot demonstrating [56] electronic transition energies of the metallic (solid symbols) $\mathrm{E}_{11}{ }^{\mathrm{M}}$ and semiconducting (open symbols) $\mathrm{E}_{22}{ }^{\mathrm{S}} \mathrm{SWNTs}$ probed with different laser energies................................................23

2-1 Scheme of a carbon arc discharge apparatus used by lijima....................44

2-2 Scheme of a laser-ablation apparatus........................................ 45

2-3 Schematic diagram of the base growth model.................................... 49

2-4 Depict of tip mechanism, the solid arrows and dashed arrows in the diagram indicate carbon species from supply and diffusion of carbon species during nanotube growth, respectively ............................................ 50

2-5 Unwanted carbon fibres were obtained in the early attempts of synthesis carbon nanotubes from ferrocene precursor at $950{ }^{\circ} \mathrm{C}$ without hydrogen................52

3-1 A diagram of a dual-furnace CVD system for synthesizing aligned MWCNTs 
3-2 SEM image of multi-walled carbon nanotubes using $\mathrm{Fe}^{3+}$-derived catalysts......69

3-3 Carbon nanotubes produced from $\mathrm{Fe}^{3+}$-derived catalyst.......................69

3-4 Low resolution SEM images of aligned carbon nanotubes using ferrocene as precursors.

3-5 SEM images of nanotubes collected from the bottom surface of the quartz plate.

3-6 SEM images of aligned carbon nanotubes on a pre-patterned $\mathrm{Si}_{3} \mathrm{~N}_{4}$ substrate.....73

3-7 TEM images of carbon nanotubes produced from ferrocene-derived catalysts...75

3-8 LRSEM images of aligned carbon nanotubes using FePC as precursors ..........77

3-9 TEM images on a bundle of nanotubes with roots attached together from FePC-

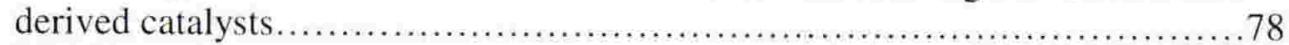

3-10 Middle sections of nanotubes from FePC-derived catalysts........................ 79

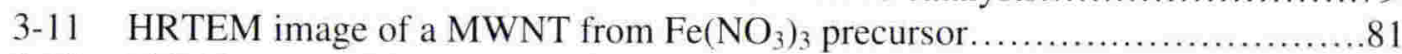

3-12 TEM images of (a) a concentric MWNT under high resolution. The white arrow indicates the impurities encapsulated inside the MWNT; (b) a MWNT section under high resolution. The approximate $25 \mathrm{~nm}$ thick wall consists of 73 shells; (c) a MWNT tip under low magnification; (d) part of the outer wall of the MWNT tip

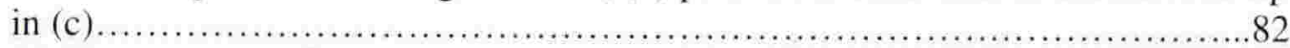

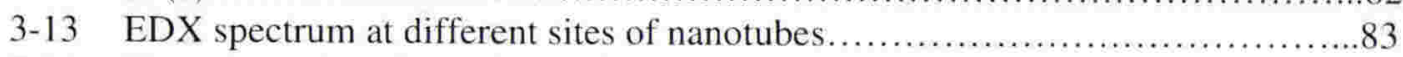

3-14 Thermogravimetric analysis of CNTs samples from ferrocene and FePC ...........86

3-15 Raman spectrum of SWNT samples....................................... 89

3-16 Raman spectroscopy on an aligned film of MWNTs from FePC .................91

3-17 Raman spectra of MWNTs from ferrocene-derived and FePC-derived catalysts................................................................... 93

3-18 "Elbow-like bend" between two pieces of straight nanotubes $(8,0)$ and $(7,1) \ldots . .94$

5-1 Behaviour of neutral and charged bodies in a non-uniform electric field with dashed lines indicating its direction

5-2 Polarisation of a cylindrical conductor in high field limit.

5-3 The plots of DEP force (a) and DEP torque (b) on a CNT versus the angle of CNT to the electric field.

6-1 Diagram of typical structures of interdigitated electrodes (IDE) ................130

6-2 Bridge arrangement for real-time impedance monitoring of nanotube collections........................................................... 132

6-3 Figure A-1 Scheme of a 4-pad structure which was used to align single-walled nanotubes........................................................... 134

6-4 (a) Optical microscope image of nanotubes from ferrocene-derived catalysts collected between electrodes, (b) Real-time conductance and capacitance changes during the collection seen in (a)

6-5 (a) Optical microscope image of nanotubes from FePC-derived catalysts collected between electrodes on a IDE, (b) Real-time conductance and capacitance changes in 24 minutes during the collection as seen in (a), (c) Real-time measurement of conductance and capacitance in an extended period of 72 minutes. 
6-6 Optical microscopy of nanotubes from FePC precursors collected on IDEs with different layouts, IDE 2 (a) and IDE 3 (b) ....

6-7 Electric field dependent behaviour of the capacitance/conductance of CNTs aligned on an IDE subjected to on/off switching of the field (30V peak across 150 microns, IDE 2)

6-8 Optical micrographs of CNTs at different stages of alignment between electrodes on IDE 2, in which the gaps between electrodes are 150 microns..............141

6-9 SEM images of nanotubes aligned between and on electrodes of IDE 1.........144

6-10 Effect of alignment of CNTs by dielectrophoresis under different frequencies.. 146

6-11 SWNT-assembly and orientation in response to an applied de electric field in THF. The left to the right sequence corresponds to an on-off-on cycle of the field....148

6-12 Frequency scan of capacitance and conductance of collection of nanotubes from FePC catalyst on IDE 1

6-13 HRSEM images of nanotubes aligned between two adjacent electrodes on a 4-pin sturcutre. (a) overview between two electrodes, (b) magnified view of nanotubes on one of the electrodes.

7-1 Apparatus of the prototype device wich aims to selectively purify as-grown CVDmade MWCNTs.

7-2 HRSEM images on MWNTs from other sources, for dielectrophoresis purpose

7-3 Photos of the large array before and after collection of CNTs

7-4 SEM images of samples (a) before being treated, (b) remained in the suspension after being treated and (c) collected on the array.....

7-5 Plot of current and voltage across the array during a continuous run............. 165

7-6 A comparison between the typical Raman spectra of as-grown samples and samples collected on the array. (b) The relative intensity of the disorder band (D band) at $1335 \mathrm{~cm}^{-1}$ over the graphitic band (G band) at $1585 \mathrm{~cm}^{-1}$ versus time of collection....

7-7 Typical Raman spectra of MWCNTs sampled from the suspension at different instants of the separation process ............................................ 167

7-8 Raman Characterization of MWCNT samples collected from different spots of

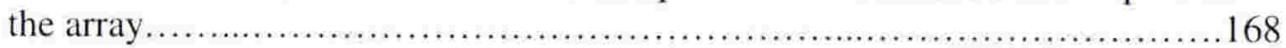

A-1 A typical set-up of an electro-spinning process.............................. 177

A-2 Depict of an electro-spinning apparatus, with a rotating drum which introduces alignment upon PEO fibres.............................................. 178

A-3 Electro-spun PEO fibres collected on the drum. (a) When the drum did not rotate; (b) when the drum rotates at $700 \mathrm{rpm}$.

A-4 An SEM image of fibres of PEO /carbon black at ratios of (a) $4: 1$; (b) $2: 1$; (c) $1: 2$ 


\section{List of tables}

1.1 Isomers made of carbon bonds between the layers................................

1.2 Vibrational modes observed for Raman scattering in SWNTs ..................21

1.3 Carbon nanotubes' applications and the properties on which they are based.....29

3.1 Data of EDX on different geometrical parts of CNTs.......................84

3.2 First and second order bands of SWNTs in Raman spectra of Figure 3-14......90

6.1 Orientations and crossover frequencies going from low to high frequencies for a homogeneous ellipsoid in an AC electric field. $\varepsilon_{1}, \varepsilon_{2}, \sigma_{1}$ and $\sigma_{2}$ represent permittivities and conductivities of the object and the media, respectively. + DEP and -DEP mean positive and negative DEP, respectively. In the table, orientation $\mathrm{a}$ is along the longest axis of the object while orientations $\mathrm{b}$ and $\mathrm{c}$ are parallel to the second longest and the shortest axis of the ellipsoid......................147

6.2 Comparison of the conductance and capacitance increases observed when CNTs $(15 \mu \mathrm{g} / \mathrm{ml})$ suspended in distilled water, absolute ethanol and absolute isopropanol were subjected to alignment by electric field. ... 


\section{Chapter 1 Introduction to Carbon Nanotubes}

Carbon materials, such as diamond, graphite, carbon fibre and activated carbon are being widely used because of their excellent comprehensive properties. Carbon nanotubes are a novel member in the carbon materials family with unique structures, intriguing comprehensive properties and promising application prospects. They have attracted tremendous research interest since they were discovered in 1991.

In structure, carbon nanotubes are pure carbon cylinders with diameters ranging from less than one nanometer to hundreds of nanometers. Carbon nanotubes are classified as single-walled and multi-walled nanotubes, depending on the number of their shells. A single-walled carbon nanotube can be viewed as a single sheet of graphite, or a graphene sheet, which is rolled into a tube, whereas a multi-walled tube contains more than one layers of graphene.

Single-walled nanotubes have some interesting quantum effects in their conducting behaviour and they can be classified as metallic tubes and semiconducting tubes, based on their conducting properties. Ballistic transport phenomenon has been observed in metallic single-walled and multi-walled nanotubes and it is attributed to their unique onedimensional structures. 
Chapter 1 Introduction to Carbon Nanotubes

This chapter reviews the classification, structure and physical properties of carbon nanotubes. The properties of nanotubes depend strongly on their helicities and geometrical structures. The superb electric and mechanical properties they demonstrated result from their unique structures and boost the extensive applications of this group of novel materials.

\subsection{Carbon-based solid materials}

Carbon is the sixth element in the periodic table and the first element of Group IV. It is the basis of organic chemistry. Carbon-based solid materials, or materials which were purely made of carbon, have been vastly utilized worldwide because of their excellent optical, electrical and mechanical properties. Besides those realized applications, carbonbased solid materials, which include diamond, graphite, carbon fibre, activated carbon, fullerene and carbon nanotubes, possess further promising application prospects and attract profound research interests.

\subsubsection{Carbon and hybridisation}

Inside a carbon atom, there are 6 electrons, $1 s^{2} 2 s^{2} 2 p^{2}$. Hybridisation of the $2 s$ and three $2 p$ atomic orbitals usually occurs in three different ways: $s p, s p^{2}$ and $\mathrm{sp}^{3}$. In the case of most carbon-based solids, there are $s p^{2}$ and $s p^{3}$ hybridisations. In $s p^{3}$ hybridisation, the four valence electrons of carbon occupy the four $s p^{3}$ bond orbits. In $s p^{2}$ hybridisation, three of the four outermost electrons participate in covalent $\sigma$ bonds, leaving the fourth electron in a $2 p_{z}$ orbital that is able to form an extended $\pi$-bonding network. $s p^{3}$ hybridisation results in tetrahedral atomic structures such as in diamond (as shown in Figure 1-1 (a)) while $s p^{2}$ forms a planar structure, which can be found in a variety of graphitic structures. Among 
the $s p^{2}$ carbon isomers, there are graphite (see Figure 1-1 (d)), carbon filament or fibre, fullerenes [91] (see Figure 1-1 (b)) and carbon nanotubes (see Figure 1-1 (c)).

(a)

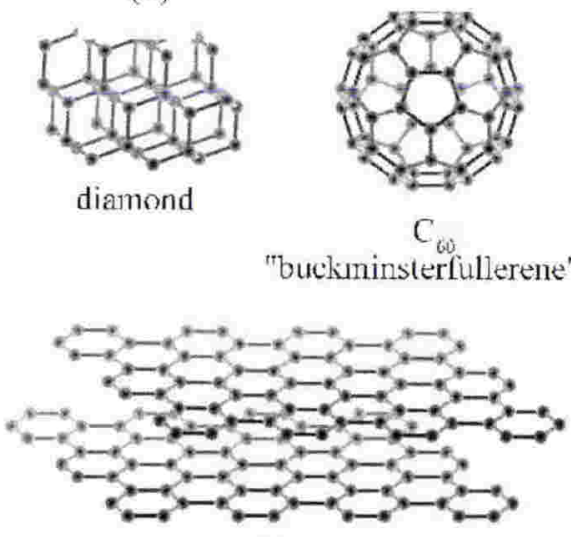

(d) graphite (b)

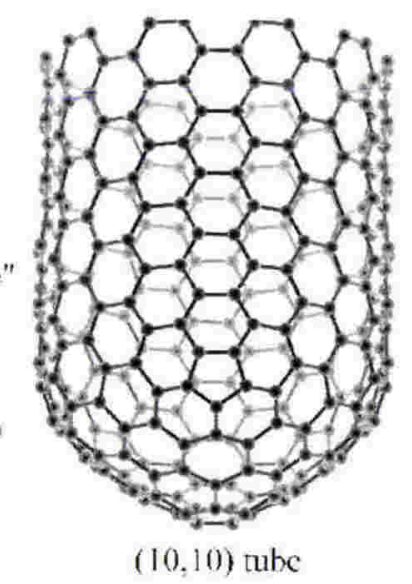

$(10,10)$ tube

(c)

Figure 1-1: Carbon-based allotropes: (a) diamond; (b) $\mathrm{C}_{60}$; (c) $(10,10)$ single-walled carbon nanotubes; and (d) graphite.

Table 1.1 Carbon isomers form structures ranging from 0-Dimensions (such as fullerenes) to 3-Dimensions

\begin{tabular}{|c|c|c|c|c|}
\hline Dimension & 0-D & 1-D & 2-D & 3-D \\
\hline Isomer & $\mathrm{C}_{60}$ & Carbon & Graphite & Diamond \\
& fullerene & nanotube & fibre & \\
\hline Hybridisation & $\left(\mathrm{sp}^{2}, \mathrm{sp}^{3}\right)$ & $\mathrm{sp}^{2}\left(\mathrm{sp}^{3}\right)$ & $\mathrm{sp}^{2}$ & $\mathrm{sp}^{3}$ \\
\hline Density & 1.72 & $1.2-2.0$ & 2.26 & 3.52 \\
{$\left[\mathrm{~g} / \mathrm{cm}^{3}\right]$} & $1.40(\mathrm{C}=\mathrm{C})$ & $1.44(\mathrm{C}=\mathrm{C})$ & $1.42(\mathrm{C}=\mathrm{C})$ & $1.54(\mathrm{C}-\mathrm{C})$ \\
\hline Bond length & & & $1.44(\mathrm{C}=\mathrm{C})$ & \\
\hline B $]$ & $1.46(\mathrm{C}-\mathrm{C})$ & & Semimetal & Insulating \\
\hline Band gap & Semiconductor & Metal or & $\mathrm{E}_{\mathrm{g}}=5.47 \mathrm{ev}$ \\
\hline
\end{tabular}


As listed in Table 1.1, carbon isomers form structures ranging from 0-Dimensions (such as fullerenes) to 3-Dimensions (such as diamond) and show great variety in their appearance and properties. This variety is attributed to the different hybridisations of their atomic orbitals and different bond structures. Of these isomers, graphite and fullerene will be reviewed briefly here because of their close resemblance to carbon nanotubes.

\subsubsection{Electronic structure of graphite}

Graphite consists of layers of hexagonal lattice sheets, or graphene sheets, as depicted in Figure 1-1 (d). Inside graphite, molecular orbitals are $s p^{2}$-hybridised. The $s p^{2}$ bonding links adjacent carbon atoms with a bond distance of $0.142 \mathrm{~nm}$ within each planar layer. However, there is only a relatively weak van der Waals force binding between layers where the interlayer distance is $0.335 \mathrm{~nm}$. The anisotropy in the structure and the weak interlayer molecular force make graphite soft and slippery.

The $s p^{2}$-hybridised orbitals form $\sigma$ valence bands and $\sigma^{*}$ conduction bands. And the $2 p_{z}$ orbitals, which do not participate in the hybridisation, are oriented in the z-direction, perpendicular to the sheet, and form a $\pi$ covalent bond, which is responsible for most of the solid-state properties of graphite. The energy dispersion diagram in Figure 1-2 displays $\pi$ bonding band and $\pi^{*}$ antibonding band.

\subsubsection{1 $\sigma$ bands of graphite}

$2 s, 2 p_{x}$ and $2 p_{y}$ orbitals hybridise into an $s p^{2}$ covalent bonding orbital for each carbon atom. Tight binding calculations on the band energy of two adjacent covalent carbon atoms [91] gave the following results: there are six $\sigma$ bands in the orbital, three of the bands are bonding $\sigma$ bands with energy levels below the Fermi energy and the other three 
are antibonding $\sigma^{*}$ bands with energy levels above the Fermi energy. The six electrons in $2 s, 2 p_{x}$ and $2 p_{y}$ orbitals occupy the three $\sigma$ bands with the lower energy than the $\sigma^{*}$ bands.

\subsubsection{2 $\pi$ band of graphite}

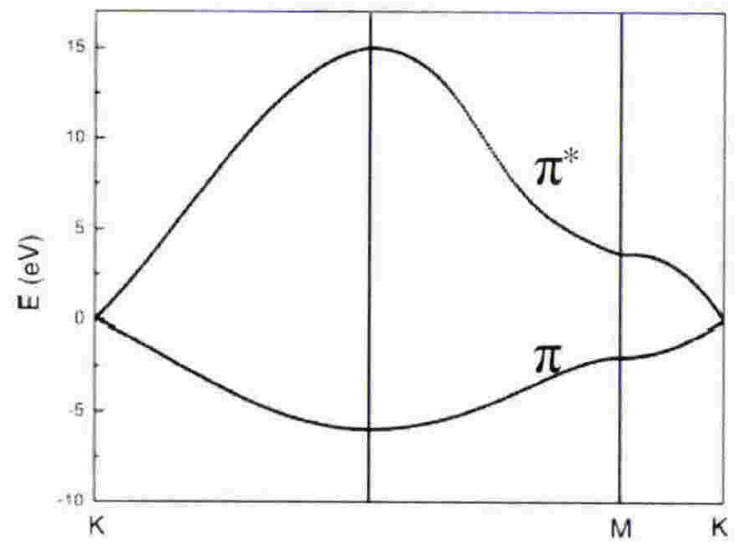

Figure 1-2: The energy dispersion band structure of a graphite sheet calculated within a $\pi$-band tight-binding model. The valence $\pi$ and conduction $\pi^{*}$ bands show degeneracy at the $\boldsymbol{K}$ points in the Brillouin zone at the Fermi level.

The $\pi$ band of graphite is of more significance than the $\sigma$ bands for the electronic behaviour of graphite. Figure 1-2 depicts only the $\pi$ valence band and the $\pi^{*}$ conduction band in the energy dispersion structure of graphene. The lower band in the dispersion describes the $\pi$ valence band and the upper band describes the $\pi^{*}$ conduction band. These two bands are degenerate at the Fermi level, at the $\boldsymbol{K}$ points, the corners of the first Brillouin zone [76, 112]. For two adjacent bonding carbon atoms in graphite, there are two $s p_{z}$ electrons, which occupy the lower $\pi$ band to make the total energy lower than $\varepsilon_{2 p}$, which is the energy level of 2 p orbital without hybridisation.

As shown in Figure 1-2, at the $\boldsymbol{K}$ point, the band gap of the graphite is zero, therefore, graphite is a semi-metal, or a zero-band gap semiconductor within its first Brillouin zone. 
Chapter 1 Introduction to Carbon Nanotubes

And in the case of carbon nanotubes, the zero-gap at the $\boldsymbol{K}$ points determines the electronic properties of carbon nanotubes.

\subsubsection{Fullerene}

Fullerenes [29] are a family of polyhedral, closed structure form of pure carbon. The name "fullerene", or "buckminsterfullerene" was given by H. Kroto and R. Smalley [61], after the reason that the molecular structure resembles the geodesic domes designed by the architect R. Buckminster Fuller. According to Euler's theorem, there must be at least 12 pentagons inside a closed cage molecule. For example, there are 20 hexagons and 12 pentagons in a $\mathrm{C}_{60}$ molecule (as seen in Figure 1.1 (b), and every pentagon is surrounded by five hexagons. In addition to the $s p^{2}$ bonding, there exist some $s p^{3}$ bondings, which are caused by the curvature structure of $\mathrm{C}_{60}$, the most common form of fullerene. There are many other forms of fullerenes, among them, $\mathrm{C}_{70}$, the structure of which can be accomplished by bisecting $\mathrm{C}_{60}$ inserting five hexagons around the equator and welding these hexagons with the two open half domes of $\mathrm{C}_{60}$. Even larger structures, such as $\mathrm{C}_{80}$ and $\mathrm{C}_{120}$, can be built under the similar constructions.

A capped cylindrical carbon nanotube can be regarded as inserting a cylinder of hexagons into a bisected $\mathrm{C}_{60}$. As shown in Table 1.1, the average carbon-carbon bond distance of fullerene is very close to that of graphite.

\subsection{The discovery of carbon nanotubes}

The discovery of $\mathrm{C}_{60}$ by $\mathrm{H}$. Kroto and R. E. Smalley [61] and the overwhelming worldwide interests in nanotechnology stimulated the pioneer works on the theoretically 
Chapter 1 Introduction to Carbon Nanotubes

proposed structure of carbon nanotubes. In 1991, Dresselhaus [30] proposed a carbon nanostructure, which holds elongated carbon tubular structure with closed fullerene domes at both ends. It did not take much time before the experimental discovery of carbon nanotube.

The groundbreaking experimental discovery of carbon nanotubes was made in 1991 by Iijima [49] when he reported the multi-walled carbon nanotube structure as "helical microtubules of graphitic carbon", supported by his experimental evidences of transmission electron microscopy and electron diffraction pattern observations. The nanotubes the discoverer identified, were 4 to $30 \mathrm{~nm}$ in diameter and up to $1 \mu \mathrm{m}$ in length. They were grown on a carbon electrode by use of arc-discharge evaporation of carbon in an argon atmosphere.

\subsection{The structure of carbon nanotubes}

Structurally, carbon nanotubes can be regarded either as the "rolled-up" graphite cylinders or as elongated fullerene cages. Carbon nanotubes are divided into two groups by the number of their shells: single-walled carbon nanotubes (SWNTs, see Figure 1-3 (a)) and multi-walled carbon nanotubes (MWNTs, see Figure 1-3 (b)).

A single-walled carbon nanotube can be regarded as being formed by a rolled-up graphene sheet with a diameter ranging from less than $1 \mathrm{~nm}$ to $10 \mathrm{~nm}$ while a multiwalled carbon nanotube, whose diameter can be as great as hundreds of $\mathrm{nm}$, is formed by two or more concentric shells. Inside multi-walled carbon nanotubes, only relative weak interactions exist between adjacent graphene layers. The interlayer distance is approximately $3.4 \AA$ [49], close to the graphite value. 

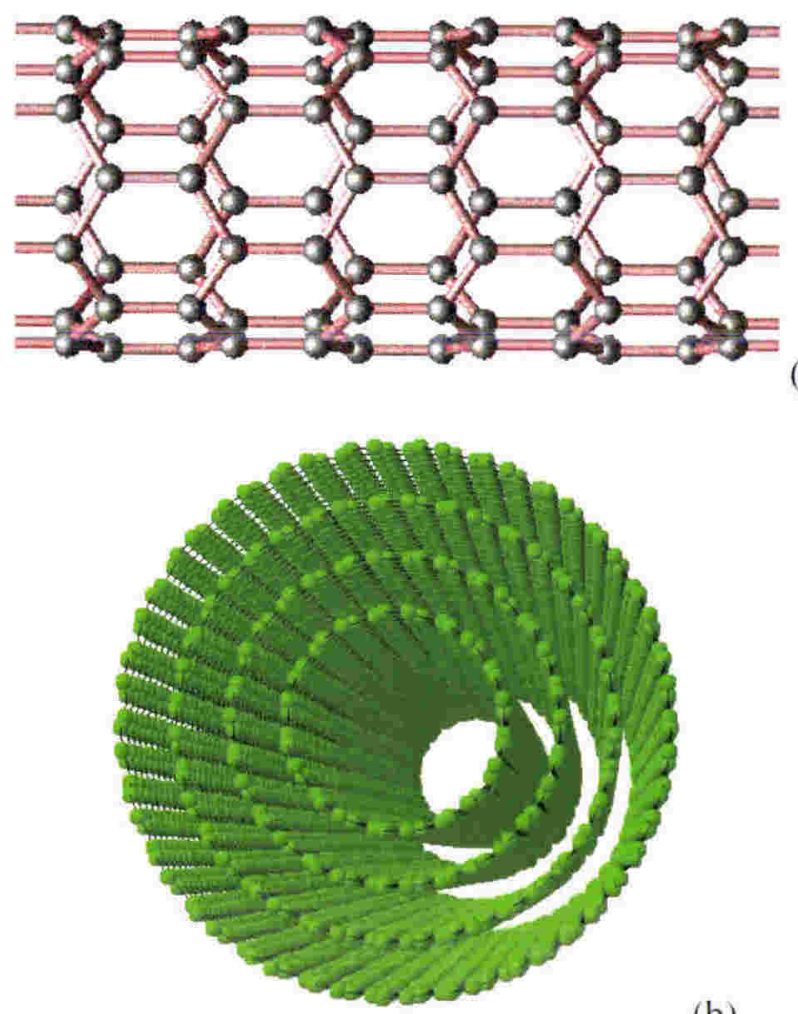

(a)

(b)

Figure 1-3: Schematic cross-sections of (a) a single-walled CNT with ten carbon atoms (shown as black dots) around the circumference and (b) a 4-layered multi-walled CNT.

This section gives a brief introduction to the structure of SWNTs. Here the description of the structure of SWNTs begins with the discussion on their chiralities. To fold a 2-D graphene sheet into a 1-D nanotube, there are a number of different orientations to be directed and as a result, SWNTs have different chiralities. The electronic structures and properties of single-walled nanotubes are determined by their chirality factors. In order to give a clear description of the chiral structure of SWNTs, some parameters must be introduced before further discussion. 


\subsubsection{Chiral vector}

As shown in Figure 1-4, a graphene sheet is wrapped into a nanotube following a way by which all of the points along the dash line AC coincide with all the points along the line

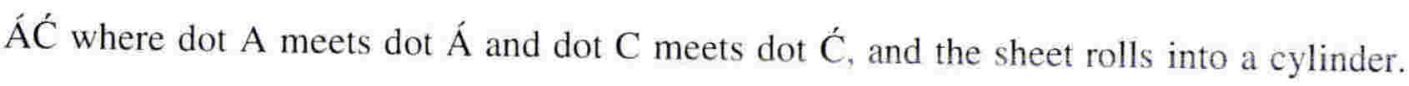

Chiral vectors are used to specify these structures.

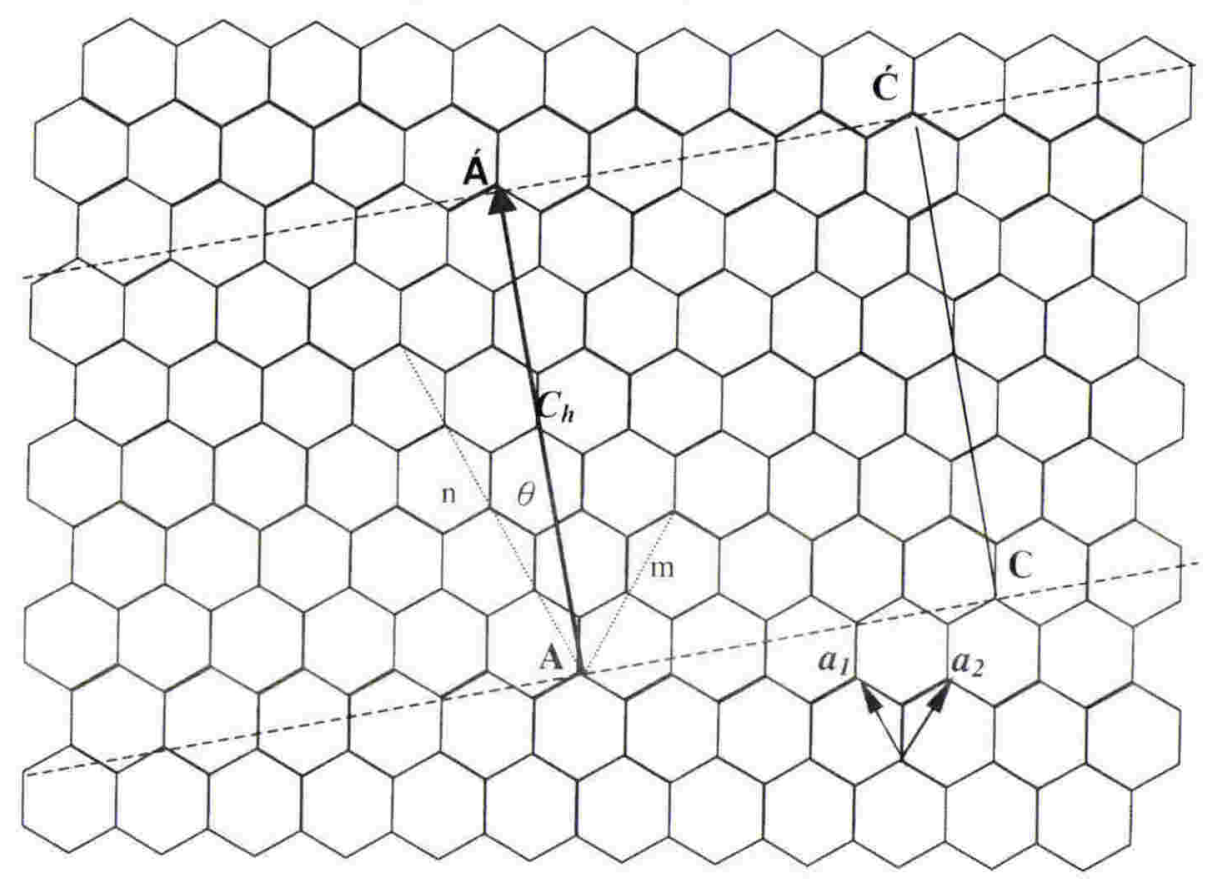

Figure 1-4: Unrolled lattice of a carbon nanotube. The chiral structure in the Figure corresponds to $(4,2)^{*}$ helicity. The section between the two parallel dash lines wraps into a nanotube by connecting points $A$ and $C$ with points $A$ and $C$, respectively. *It should be clarified that the $(4,2)$ nanotube in this figure is for demonstrating purpose only and it doesn't exist
in reality because of the great curvature.

The vectors $\boldsymbol{a}_{\boldsymbol{l}}$ and $\boldsymbol{a}_{2}$ as shown in Figure 1-4 are real space unit vectors of the hexagonal lattice. Taking a C-C bond distance as $\mathrm{a}_{\mathrm{c}-\mathrm{c}}(1.44 \AA)$, the lengths of $\boldsymbol{a}_{1}$ and $\boldsymbol{a}_{2}$ are $\sqrt{3} \mathrm{a}_{\mathrm{c}-\mathrm{c}} / 2$. The chiral structure of a single-walled carbon nanotube, in another word, the orientation in which a graphene sheet is wrapped into a nanotube, can be specified by chiral vector, 
$C_{h}$, which is defined as the vector perpendicular to the axis of the resulting nanotube and can be written as

$$
C_{h}=\mathrm{n} a_{1}+\mathrm{m} \boldsymbol{a}_{2} \equiv(\boldsymbol{n}, \boldsymbol{m}),(\mathrm{n}, \mathrm{m} \text { are integers, } 0 \leq \mathrm{m} \leq \mathrm{n})
$$

The values of $\mathrm{n}$ and $\mathrm{m}$ are very important in determining the properties of carbon nanotubes. Other parameters of carbon nanotubes can be calculated from the values of chiral vectors. The circumference of nanotube, expressed as $\mathrm{L}$, can be calculated from the chiral vector,

$$
\mathrm{L}=\mathrm{a}_{\mathrm{c}-\mathrm{c}} \sqrt{n^{2}+m^{2}+n m}
$$

The diameter of nanotube, expressed as $d_{t}$, can then be given as

$$
d_{t}=\mathrm{a}_{\mathrm{c}-\mathrm{c}} \sqrt{n^{2}+m^{2}+n m} / \pi
$$

\subsubsection{Chiral angle}

The chiral angle, $\theta$, is defined as the angle between the vectors $\boldsymbol{C}_{\boldsymbol{h}}$ and $\boldsymbol{a}_{\boldsymbol{l}}$. Based on the geometry of the nanotube and the definitions of the chiral parameters, the value of $\theta$ must be between $0^{\circ}$ and $30^{\circ}$.

$$
\sin \theta=\frac{\sqrt{3} m}{2 \sqrt{n^{2}+m^{2}+m n}}
$$

In the wrapping sheet of Figure $1-4$, the chiral vector is $(4,2)$ and chiral angle is approximately $19.1^{\circ}$.

In an ideal structure of SWNT, two domes of half fullerenes meet the opening cylinder ends. The chirality and the diameter of the domes follow those of the SWNTs, giving a seamless cylinder. 


\subsubsection{Classification of carbon nanotube}

As seen in Figure 1-5, single-walled carbon nantubes are classified on their chiralities. The three types of nanotubes shown from the top to the bottom in Figure 1-5 are armchair, zigzag and chiral, respectively. The names of armchair and zigzag are given on the shapes of their cross sections. Armchair, zigzag and chiral nanotubes have chiral vectors of $(n, n),(n, 0)$ and $(n, m)$ where $n \geq m$, respectively. Their chiral angles are $30^{\circ}, 0^{\circ}$ and $0^{\circ}<\theta<30^{\circ}$, respectively.

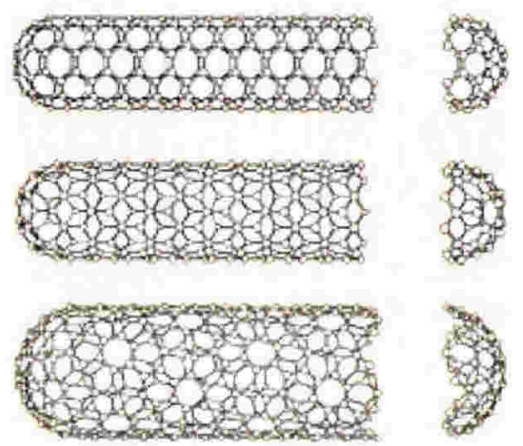

Figure 1-5: Classification of single-walled carbon nanotubes (Saito [91]). The top: armchair layout, chiral vector $(n, n)$; the middle: zigzag layout, $(n, 0)$; and the bottom: chiral layout, $(\mathrm{n}, \mathrm{m})$.

\subsection{Electronic properties of carbon nanotubes}

The electronic properties are the most intriguing characteristics of carbon nanotubes. Quantum effects have been observed in the electronic properties of CNTs. The conductive property of a CNT depends strongly on both the diameter and the chirality of the hexagonal carbon lattice along the tube because the two-dimensional electronic bands of a semimetallic graphene (see Figure 1-2) do not necessarily degenerate along the onedimensional structure of a CNT after being wrapped. 
The predictions of the electronic structure of carbon nanotubes were based on theoretical calculations, and were made from different sources $[47,77,88,91,92]$. The electronic band structures of single-walled carbon nanotubes from calculations, quoted from reference [91], are given in Figure 1-6. Summarizing these predictions, there are several key points which need to be addressed here:

1. SWNTs with $(\mathrm{n}, \mathrm{n})$ chirality indices are truly metallic. As shown in Figure 1-6 (a), the lowest conduction band and the highest valence band cross at the Fermi level, leading to a degenerate electronic density of states.

2. SWNTs with $(n, m)$ indices and $n-m=3$ are semimetallic with zero band gap. As shown in Figure 1-6 (b), the lowest conduction band and the highest valence band are degenerate where they met at the Fermi level. Their electronic density of states is non-zero at the Fermi level.

3. SWNTs with $(n, m)$ indices are semiconducting when $n-m \neq 3 \times$ integer. As shown in Figure 1-6 (c), the lowest conduction band and the highest valence band are not degenerate across the Fermi level, and there is an open gap between the two bands [78], expressed as $\mathrm{E}_{\text {gap }}$,

$$
\mathrm{E}_{\text {gap }}=2 \gamma_{0} a_{c-c} d d_{t},
$$

$a_{c-c}$ is the C-C distance and $d_{t}$ is the tube diameter, $\gamma_{o}$ is the nearest overlap tight binding energy.

Their electronic density of states is zero at the Fermi level.

These predictions have been supported by experimental observations using a highresolution scanning tunnelling microscope (STM). In a STM experiment $[48,67,82,106]$, a STM tip is kept very close to the sample and a voltage (V) can be scanned on an 
individual carbon nanotube sample. The tunnelling current (I) between the tip and the sample is very sensitive to the surface atomic geometry and the local density of states which can be measured by $\mathrm{d} / \mathrm{dV} /(\mathrm{I} / \mathrm{V})[101]$. STM can then reveal the atomic structures of the tube's geometry and correlate the information of chirality and diameter, and the intrinsic transport property of the nanotube.
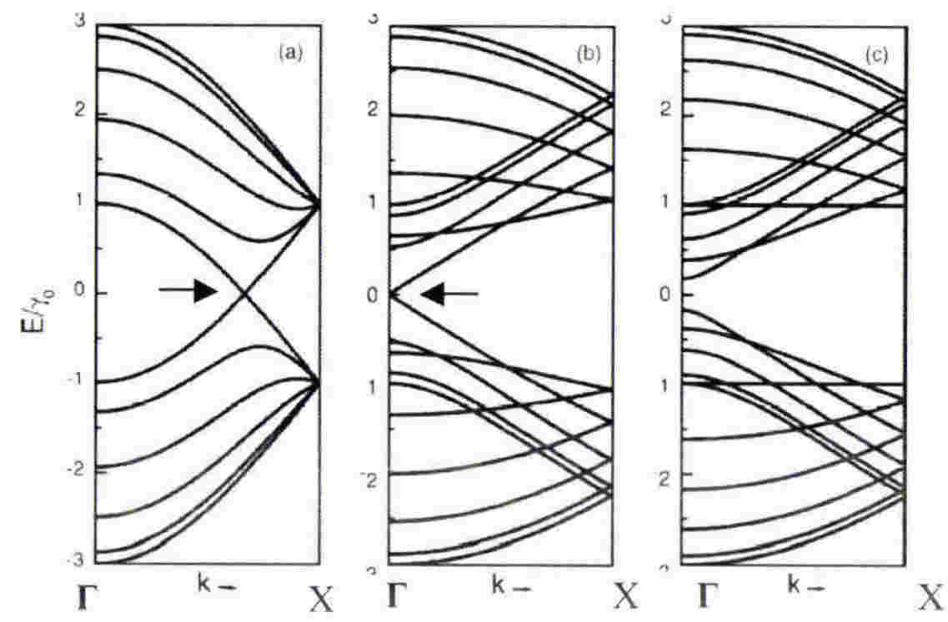

Figure 1-6: Electronic band structure versus wave vector of single-walled nanotubes with chiral indices of $(5,5),(9,0)$ and $(10,0)$, from the left to the right, from theoretical calculation derived from the band structure of graphene. The two arrows point to where the degeneracy happens. Fermi level was shown as $\mathrm{E}=0$ in the plots. On the k (wave vector) axis, point $\lceil$ represents $k=0$, point $X$ in (a) represents $k= \pm \pi / a$, in (b) and (c) represents $\mathrm{k}= \pm \pi / \sqrt{3}$ a. Reprinted from [85].

STM investigations $[82,106]$ also support the relation between the gap energy and tube diameter for a semiconductive carbon nanotube and provide sound support for the $1 \mathrm{D}$ energy band structure.

The measurement of transport properties of individual nanotubes was an experimental difficulty until 1996 when Langer [64] and Lieber [23], using different experimental setups, independently reported their measurements on electrical properties of individual multi-walled carbon nanotubes and single-walled nanotubes. In their experiments as well 
as later reports $[9,33,40]$, conductivity measurements were made after nanotubes bridged across two-, four- and multi-probes. These reported measurements not only confirmed with theoretical predictions, but also revealed some interesting phenomena in electric transport properties of nanotubes.

\subsubsection{Metallic carbon nanotubes as quantum wires}

About one-third carbon nanotubes are metallic. Metallic carbon nanotubes have been named as "molecular quantum wires" [26] because they exhibit finite conductance which are independent of their lengths.

\subsubsection{Single electron tunnelling and Coulomb blockade}

Coulomb blockade refers to the blocking of electron transports through a nanoscale "island" separated by tunnelling barriers from conductors on either side, due to repulsive Coulomb interactions between individual electrons. Single electron tunnelling is an interesting phenomenon in such systems where the tunnelling of electrons occurs one by one through the island due to Coulomb blockade. Single electron tunnelling and Coulomb blockade in CNTs have been observed by Bockrath et al. [9]

\subsubsection{Ballistic transport}

Ballistic transport refers to the situation in which the length of a conductor is less than the electron mean free path. In this case electrons can traverse the conductor without scattering and the conductance of the material is determined solely by the density of states at the Fermi edge. As such the conductance of a ballistic conductor is independent of the conductor length and the applied voltage. The ballistic transport conductance between two terminals in a $\mathrm{N}$ channel 1D system can be expressed by the LandauerButtiker Formula [112], 
Chapter 1 Introduction to Carbon Nanotubes

$$
\mathrm{G}=\frac{2 e^{2}}{\mathrm{~h}} \sum_{n=1}^{N} T_{i}
$$

where $T_{i}$ is the transmission coefficient of electrons through the sample, which is equal to 1 in the case of ballistic transport of electrons. $\mathrm{N}$ is the number of transmission channels. Ballistic transport has been reported [84] in a multi-walled carbon nanotube system. This phenomenon was observed in the experimental setup, as shown in Figure 1-7 (a). As the MWNT bundles are lowered into liquid mercury, contact will initially be made with just one of the nanotubes (see inset of Figure 1-7 (a)). As MWNTs are dipped further into the liquid metal contact (mercury in this case), the conductance increases in steps of $\mathrm{G}_{0}=$ $2 \mathrm{e}^{2} / h$, as shown in Figure 1-7 (b). These steps correspond to sequential individual nanotubes coming into contact with the liquid metal such that the channel number, $\mathrm{N}$, increases as the bundle is lowered into it. The finite conductance of each individual nanotube, which is independent of conduction path length, is characteristic of ballistic transport and has been observed in metallic single-walled nanotubes [59] and multiwalled nanotubes [84]. This behaviour is characteristic of ballistic transport of electrons, which means that the transport of electrons within the dimension of the sample under measurement is not being scattered by crystal lattice wave, or phonons.

The result in the reference [84] showed that the nanotubes are not ohmic conductors with resistances that obey Ohm's law and are proportional to the length of the conductor; in fact, the conductance of the CNT bundle is independent of its length (from the contact with the liquid to the interface from which the bundle extruded) being measured within each step shown in Figure 1-7 (b). It implies that ballistic transport occurs in carbon nanotubes at the room temperature. 

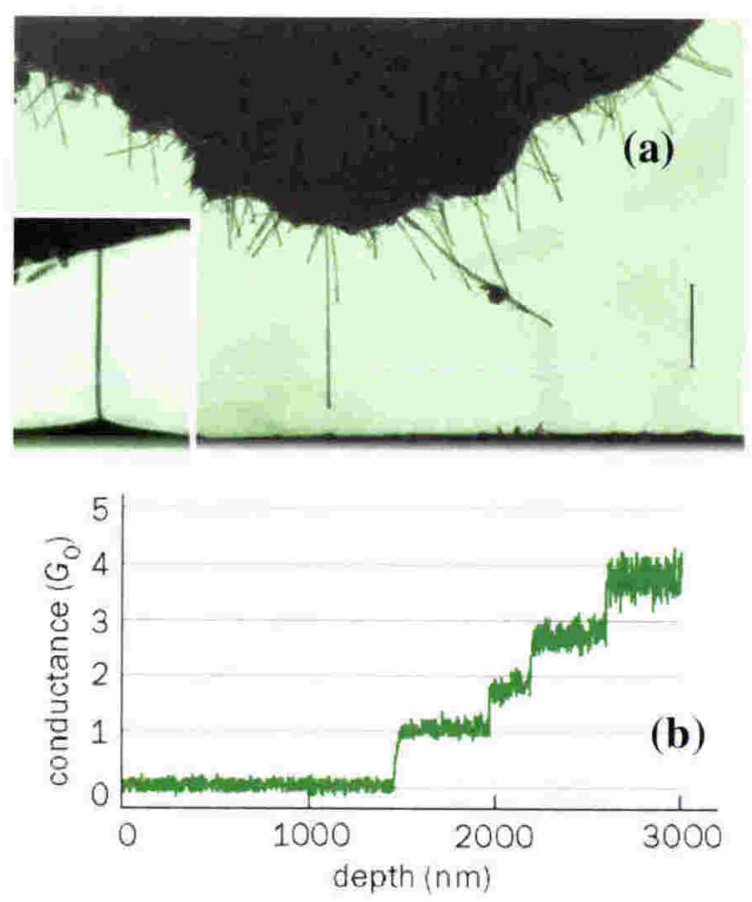

Figure 1-7: (a) Ballistic transport through a multi-walled nanotube bundle (W. de Heer et al. [84]). A transmission electron microscope image of the multi-walled nanotubes used by Walt de Heer et al. to measure the conductance of a MWNT bundle. The scale bar represents $10 \mu \mathrm{m}$. Inset: contact made by a MWNT between the MWNT bundle and mercury. (b) A plot of the conductance versus the depth of the nanotube section in the mercury at the room temperature.

The existence of ballistic transport means that metallic carbon nanotubes can support very high current density without large heat dissipation because in such a scenario the charge carriers traverse without being scattered. A current density of $2.5 \times 10^{9} \mathrm{~A} / \mathrm{cm}^{2}$ has been demonstrated in a $1 \mathrm{~nm}$ diameter tube [76]. This value is of many orders higher than a copper wire with the same diameter. It has been reported recently that the current passing through a $15 \mathrm{~nm}$ wide multi-walled nanotube can reach $675 \mu \mathrm{A}$ [69]. 


\subsubsection{Semiconducting nanotubes}

The semiconducting property of single-walled carbon nanotubes was first reported by $\mathrm{C}$.

Dekker et al. [103] in 1998. This property has been utilized to build semiconducting SWNT (s-SWNT)-based field effect transistors and chemical sensors. High carrier mobility was found with s-SWNTs.

\subsubsection{Semiconducting nanotube-based field effect transistors}
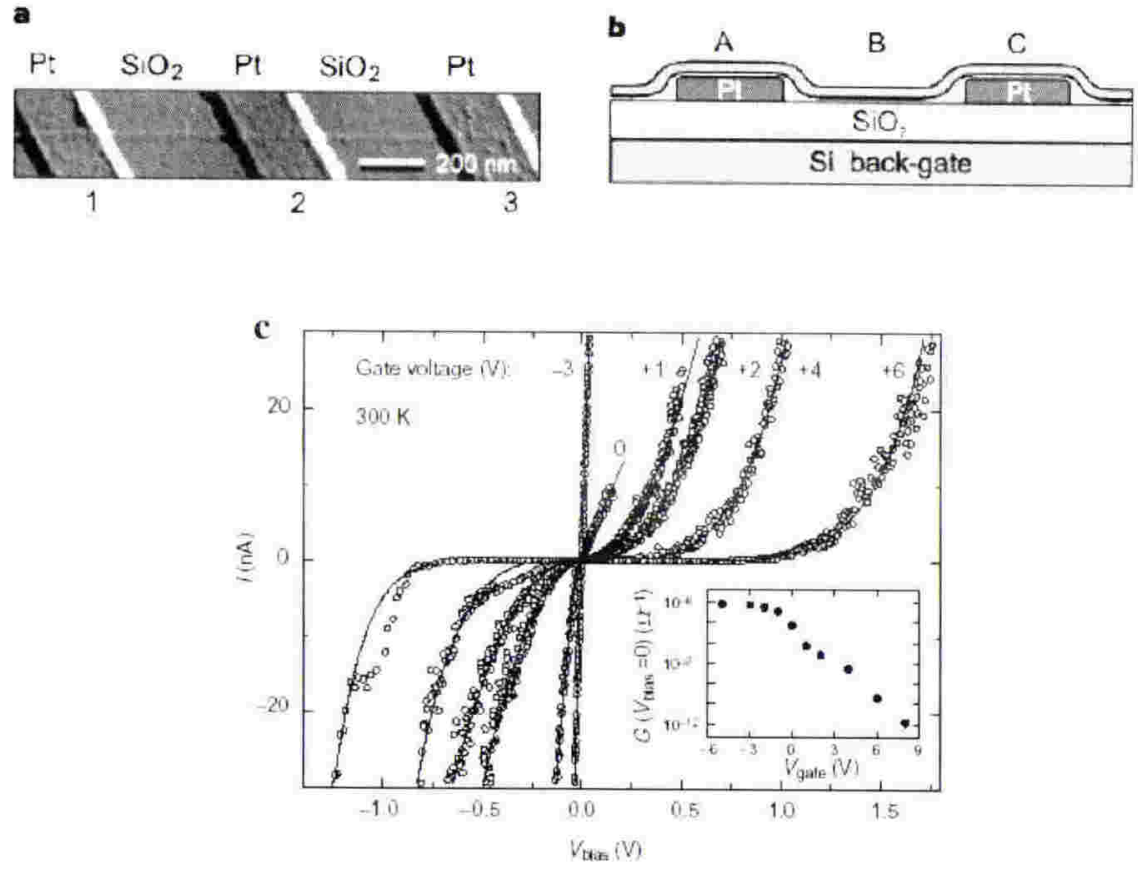

Figure 1-8: (a) An AFM image of an individual carbon nanotube on the top of three Pt electrodes. (b) Schematic side view of the nanotube-based field effect transistor (FET). (c) $\mathrm{I}-\mathrm{V}_{\text {bias }}$ characteristic of the nanotube-based FET at different voltages. Inset: the plot of conductance of nanotube-based FET versus gate voltage [103].

In the experimental setup of Figure 1-8 (a), a single-walled carbon nanotube contacted source and drain electrodes on the top of $\mathrm{SiO}_{2}$, which acted as an insulating layer, with $\mathrm{Si}$ as a back gate underneath. The measurement of the current - bias voltage at different gate voltage (see Figure 1-8 (c)) under ambient condition is analogous to p-type MOSFETs. 
Chapter 1 Introduction to Carbon Nanotubes

After this innovative work [103], studies related with nanotube-based FETs were extensively conducted. The inset of Figure 1-8 (c) shows the nanotube-based FET is a ptype transistor. The p-dopants were from oxygen in the air.

Since then, extensive work on nanotube-based FETs has been under way $[42,46,54,62$, $75,107,110]$. Carrier mobility of nanotubes as high as $10,000 \mathrm{~cm}^{2} / \mathrm{Vs}$ was then reported [76]. In bulk silicon [39], with modest doping, the mobility of the electrons at room temperature is about $1500 \mathrm{~cm}^{2} / \mathrm{Vs}$. N-type transistors can be built by doping semiconducting nanotubes with alkali metal $[10,27,60]$ to add negative carriers to the transistor, by insulating the CNT from positive donors [19] with a layer of PMMA.

\subsubsection{Genre of semiconducting nanotubes, p-type or n-type?}

In air, semiconducting nanotubes without any intentional doping are p-type. This is due to the extreme sensitivity of nanotubes to oxygen. The oxygen species absorbed by nanotubes are strong electron acceptors and positive charge donors to nanotubes, leading to their p-type behaviour. However, Collins et. al. [19] found that the intrinsic genre of semiconducting CNTs is n-type when measuring the thermoelectric power of a semiconducting CNT in vacuum after it was deprived of oxygen.

\subsubsection{Nanotube-based chemical sensors}

The conductance of semiconducting CNTs can be changed drastically and swiftly when they are exposed to an environment of charge donors or acceptors. Derycke et al. [28] have found that the position of the Fermi level at the CNT-metal interface of a nanotubebased FET device can change gradually within the band gap as a function of the concentration of oxygen. 
Chapter 1 Introduction to Carbon Nanotubes

CNT-based FETs can be fabricated as nanometre-sized gas sensors [11, 12, 58, 104, 105, 110] by taking advantage of this charge concentration-sensitive behaviour of CNTs. The sensing mechanism can be described as: upon the contact between electron donating species, the injection of electrons from the gases into CNTs will decrease the numbers of positive carriers and shift the valence band of the CNTs away from Fermi level, resulting in hole depletion and reduced conductance. On the other hand, exposure of CNTs to electron accepting gases will shift the valence band of CNTs closer to Fermi level and reduce the resistance.

Another nanotube-based notable gas sensor is based upon multi-walled nanotube-based electron emitters $[70,79]$. This type of sensors utilizes the fact that different gases have unique breakdown voltages. The MWNT arrays work as the anode in an electron emitter, The device with MWNTs exhibits higher discharge current at a lower breakdown voltage compared with a similar device without MWNTs. By picking up the distinct breakdown voltages on different gases, the MWNTs-based sensors offer swift response speed and ultra-sensitivity at low gas concentration.

\subsubsection{CNT-based nanosize devices}

Shrinking the size of electronic devices is a key goal being pursued in microelectronic materials and device industry. Apart from the sensing application mentioned above, nanotubes are also promising candidates to replace silicon for some computing purpose because of the nanometer dimension and excellent electrical and thermal conductive properties. After p- and n- type transistors were built on nanotubes, logic gates [5], inverter [51, 72] and memory [17], all based on nanotube FETs, have been fabricated 
Chapter 1 Introduction to Carbon Nanotubes

with favourable characteristics. Other CNT-based devices, such as Schottky diode [43], p-n junction [115], p-n-p junction [56] and random access memory[5], have also been achieved [90]. In another report [20], a SWNT-based memory device exhibited an extraordinarily high charge storage stability of more than 12 days at room temperature.

Other interesting transport and magnetic phenomena, such as the Kondo effect [81] in one-dimensional host of magnetic clusters on single-walled nanotube, Aharonov-Bohm oscillations in carbon nanotubes [6] and Luttinger liquid in the conducting behaviour of SWNTs, have also been found in nanotube-based systems. A review of these topics falls outside the remit of this thesis.

\subsection{Raman scattering of carbon nanotubes}

When light is inelastically scattered from a crystal, photons either gain or lose energy. The change in photon energy results from interactions with electrons and vibration of crystal lattices, or phonons. If the photons have optical wavelengths, the scattering is of Raman type. By measuring the frequency of the scattered photons relative to the incident photons, Raman spectroscopy gives the frequency of the phonons involved in the scattering process and provides information of the vibrational modes of molecular structures.

In Raman spectroscopy, a highly monochromatic laser is used as the incident light source. And there are Stokes and anti-Stokes scattering events where a phonon is emitted and absorbed, respectively. In this thesis, Raman spectra have been obtained solely by the stronger Stokes scattering events. Raman spectra consist of discrete peaks in a plot of 
Chapter 1 Introduction to Carbon Nanotubes

scattered intensity versus Raman shift, expressed in the unit of wavenumber (the frequency divided by the velocity of light).

Because of the specific spatial configurations, nanotubes have a unique scattering behaviour, which makes Raman spectroscopy a very powerful tool for structural characterization [31] of carbon nanotubes.

Raman studies on samples can be conducted on both bulk samples and an individual nanotube by proper experimental setups. Another advantage Raman studies hold is that they can be conducted in situ and sample treatment is not required, except for some special cases.

Differences exist between the Raman spectra of SWNTs and MWNTs. A brief summary of Raman spectra of the two separate classes is presented here.

\subsubsection{Raman spectra of SWNTs}

Table 1.2 Vibrational modes observed for Raman scattering in SWNTs

\begin{tabular}{ccl}
\hline Notation & Frequency $\left(\mathrm{cm}^{-1}\right)$ & \multicolumn{1}{c}{ Type of mode } \\
\hline RBM & $\mathrm{C}_{1} / \mathrm{d}_{1}+\mathrm{C}_{2}{ }^{*}$ & In phase radial displacement \\
D-band & $\sim 1350$ & Defect-induced, dispersive \\
G-band & $\sim 1580$ & Graphite-related optical mode \\
G'-band & $\sim 2700$ & Overtone of D-band, highly dispersive \\
\hline${ }^{*} \mathrm{C}_{1}=223.5 \mathrm{~nm} \mathrm{~cm}^{-1}$ and $\mathrm{C}_{2}=12.5 \mathrm{~cm}^{-1}[63]$ &
\end{tabular}

Among various phonons, of the greatest importance for the SWNT characterization are: the radial breathing mode (RBM), the tangential G-band (derived from the graphite-like in-plane mode), the disorder D-band, and their second-order bands, the G' band and D' 
band. A typical Raman spectrum of SWNT is given in Figure 1-9. The assignments of these peaks were listed in Table 1.2.

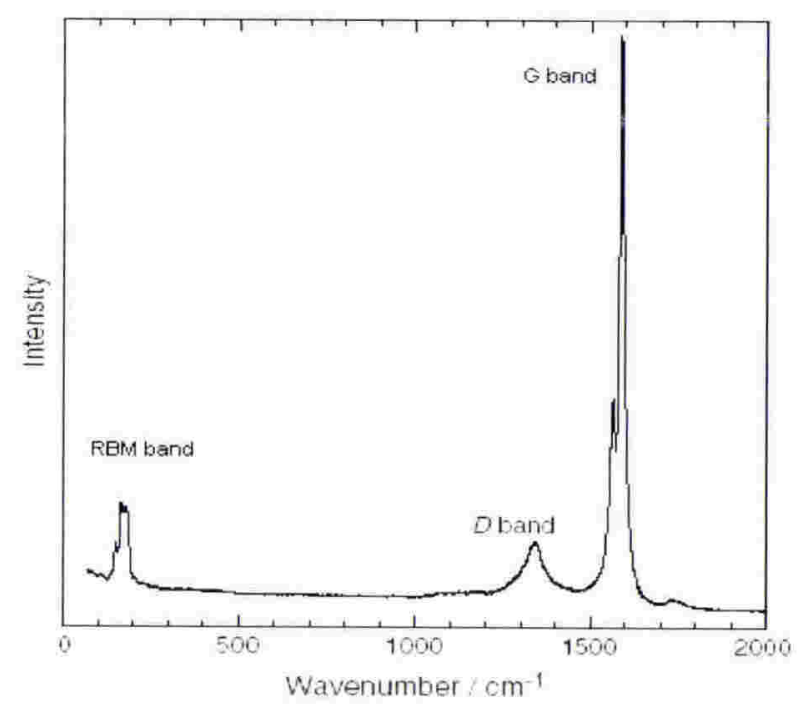

Figure 1-9 A Raman spectrum of arc discharge-grown SWNTs [73].

One of the key characteristics of Raman spectra of SWNTs is the resonant Raman effect. Here, the Raman scattering signal increases in intensity when the exciting laser wavelength resonates with the band gap, which can be determined by the van Hove singularities in the 1D joint density of states (JDOS) of the SWNT. Resonant Raman characterisation of SWNTs [53] has been used to reveal the diameter of nanotubes by identifying the band energy difference, $\mathrm{E}_{\mathrm{ii}}$ of a given SWNT.

Polarization effect has been observed in the Raman spectra of CNTs $[52,87]$. By changing the linear polarization of the incident laser with respect to the tube axis, the structural anisotropy of the nanotubes leads to a polarization dependence on the Raman signal intensity. The polarization effect has been used to investigate the alignment of carbon nanotubes [87]. 


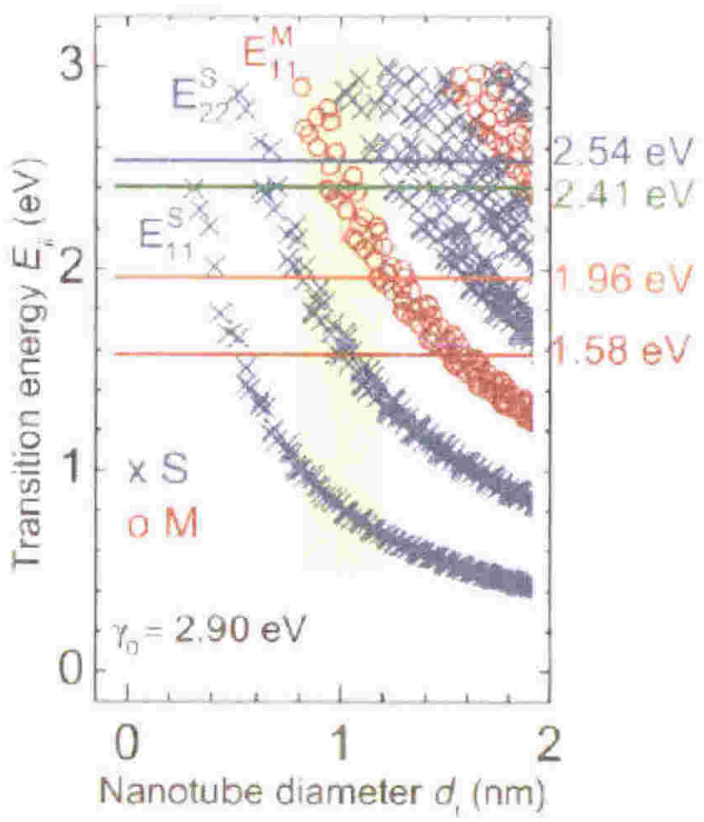

Figure 1-10 A Kataura plot demonstrating [63] electronic transition energies of the metallic (solid symbols) $\mathrm{E}_{11}{ }^{\mathrm{M}}$ and semiconducting (open symbols) $\mathrm{E}_{22}{ }^{\mathrm{S}}$ SWNTs probed with different laser energies. The horizontal lines stand for the 1.58, 1.96, 2.41 and $2.54 \mathrm{eV}$ laser energies used in their experiments.

\section{RBM}

The radial Breathing Mode ( $\mathrm{RBM}$ ) is associated with the radial breathing vibration around the circumference of CNTs. RBM modes in Raman spectra of SWNTs can be found at low frequencies.

There is a trigonal warping effect of the contours of graphite, which means that the equienergy contours of graphite are circles near the $\boldsymbol{K}$-point and near the centre of the Brillouin zone. The contours are straight lines near the boundary of the Brillouin zone. The trigonal warping effect causes a distribution of the interband transition energy $E_{i i}\left(d_{t}\right)$ between the singularities in the JDOS for nanotubes with a diameter $\mathrm{d}_{\mathrm{t}}$. When the energy of the excitation laser is in exact resonance with $\mathrm{E}_{\mathrm{ii}}$ of an individual SWNT, a very high intensity of the corresponding $\omega_{\mathrm{RBM}}$ will be observed in the Raman spectrum. Thus by 
using lasers with different wavelengths to resonate with $\mathrm{E}_{\mathrm{ii}}$, further information about the diameters and helicities of SWNTs can be revealed.

The calculation of the RBM frequency against the diameter of SWNTs has been accomplished by Kataura et al. [63]. A plot of $E_{i i}$ versus $d_{1}$, was established and given the name "Kataura plot", as seen in Figure 1-10. A precise assignment [4] of the peaks of RBMs by use of the empirical dependence of the RBM frequency on the tube radius $\mathrm{R}$ is given as:

$$
\omega_{\mathrm{RBM}}=\mathrm{C}_{1} / \mathrm{d}_{1}+\mathrm{C}_{2},
$$

where $C_{1}=223.5 \mathrm{~nm} \mathrm{~cm}^{-1}$ is the factor of the relation between the RBM frequency and the diameter of the nanotube and $\mathrm{C}_{2}=12.5 \mathrm{~cm}^{-1}$ accounts for the inter-tube interaction in bundles. According to the equation (1-7), the radial breathing frequency of SWNTs decreases as their diameters increase.

\section{G-band}

The G-band consists of in-plane bond-stretching modes, which originate from the $\mathrm{E}_{2 \mathrm{~g}}$ inplane stretching mode in graphene. In nanotubes, due to the folding of the Brillouin zone from graphite to nanotube and the symmetry-breaking effects associated with the nanotube curvature, the G-band in nanotubes contains several modes with different symmetries.

The feature near $1620 \mathrm{~cm}^{-1}$ [87] is quite pronounced in the spectra for MWNTs and is associated with the $2 \mathrm{D}$ phonon density of states in graphene. This peak does not appear in the spectra of SWNTs.

One of the interesting features of the G-band is the qualitative differences in lineshapes between the G-band of metallic and semiconducting SWNTs. Semiconducting SWNTs 
Chapter 1 Introduction to Carbon Nanotubes

show two dominant peaks in narrow Lorentzian features, the lower $\left(\omega_{G}^{-}\right)$of which is assigned to the vibrations along the circumferential direction and the higher $\left(\omega_{G}^{+}\right)$of which is assigned to the vibration along the nanotube axis. On the other hand, metallic SWNTs show two components, with upper frequency peak $\left(\omega_{G}^{+}\right)$as sharp Lorentzian features and the lower component $\left(\omega_{G}^{-}\right)$that is broadened partly due to the Breit-WignerFano effect. Due to the assignment of $\omega_{G}^{+}$and $\omega_{G}^{-}, \omega_{G}^{+}$is independent of $d_{t}$ while $\omega_{G}^{-}$has strong dependence on $d_{t}$. This theory has been supported from experimental data [83]. The correlation between $\omega_{G}^{-}, \omega_{G}^{+}$and $d_{t}$ was shown to be a good fit to the following model:

$$
\omega_{G}^{-}=\omega_{G}^{+}-C / d_{t}^{2}
$$

where $C$, a constant, equals to $47.7 \mathrm{~cm}^{-1} \mathrm{~nm}^{2}$ for semiconducting nanotubes and equals to $79.5 \mathrm{~cm}^{-1} \mathrm{~nm}^{2}$ for metallic nanotubes. The equation above will be particularly useful in determining $d_{1}$ when RBM feature is not available.

3. D-band and G'-band

The D-band is associated with the presence of disorder or finite size in $s p^{2}$ carbons. It has been reported that in $s p^{2}$ carbon-based materials which are similar to carbon nanotubes, such as graphite and carbon fibres, the relative intensity of the peak around $1350 \mathrm{~cm}^{-1}$ to the strong G mode at $1582 \mathrm{~cm}^{-1}$ is sensitive to the lowering of the crystal symmetry of 3D graphite and the amount of disorder in carbon fibres.

The band near $2700 \mathrm{~cm}^{-1}$ is termed the G'-band. It is assigned to the dispersive phonon of the second-order overtone band of D-band. These two bands have been identified with a 
Chapter 1 Introduction to Carbon Nanotubes

resonance process between a phonon and an electronic transition between linearly dispersive $\pi$ and $\pi^{*}$ states.

A linear relation has been found between $\omega_{\mathrm{G}}$ and $\mathrm{E}_{\mathrm{laser}}$ as

$$
\omega_{\mathrm{G}^{\prime}}=2420+106 \mathrm{E}_{\text {laser }}
$$

The D-band in graphite involves scattering from a defect, which breaks the basic symmetry of the graphene sheet, and is observed in $\mathrm{sp}^{2}$ carbons containing vacancies, impurities, or other symmetry-breaking defects. On the other hand, the second-order G'band $\left(\omega_{G}\right)$ is a two-phonon process and from an inelastic phonon emission process. As a result, the G'-band does not require an elastic defect-related scattering process, and is observable for defect-free $\mathrm{sp}^{2}$ carbons.

\subsubsection{Raman spectra of MWNTs}

The distinct RBM mode in the Raman spectra of SWNT is not observed in MWNTs. It has been reported $[37,89]$ that the intensity of the RBM mode drops rapidly with increasing diameter, and the intensity of the RBM mode for most MWNTs is negligibly small. MWNTs contain at least two concentric shells with large diameters. The outermost shell shields the radial breathing signals emitted from the inner shells due to the screening effect, and the interaction between shells leads to suppression of the signals, so there will be no significant MWNT RBM mode. However, noticeable RBM mode has been identified in RBM spectra of double-wall carbon nanotubes (DWNTs) [18, 34, 86] with diameters of less than $2.8 \mathrm{~nm}$.

There are D, D', G and G' modes observed in the Raman spectra of MWNTs as in the case of SWNTs. 
1. D mode

D line in other carbon-based solid materials has been attributed to the disorder-induced features due to the finite particle size effect or lattice distortion.

The D mode (a strong peak around $1335 \mathrm{~cm}^{-1}$ ) is a signature of the presence of disorder in graphitic systems caused by the defects of the six-fold symmetry within graphite layers. It can be attributed to first-order scatterings from a zone-boundary phonon activated by the disorder associated with finite crystalline size. However, as indicated by A. Rao et al. [89], the D band intensity may also be caused by the polarization effects which occur within aligned multi-walled nanotube bundles and not necessarily arise only from disorder within graphite-like domains. Also, the D band responds to the disorder in amorphous carbon.

2. G mode and D' mode

The line in the Raman spectra of MWNTs below $1600 \mathrm{~cm}^{-1}$ has been resolved to three peaks. As in the Raman spectra of SWNTs, the G mode of MWNTs, which consists of $\omega_{G}^{-}$and $\omega_{G}^{+}$, is related to the crystalline domain in MWNTs. The $\omega_{G}^{-}$is assigned to vibrations along the circumferential direction and the $\omega_{G}^{+}$is assigned to vibrations along the nanotube axis. The splitting between the two components is due to the curvature of nanotubes, and as a result, this value should be affected inversely by diameter. It is much less than the gap in SWNT spectra. However, there is lack of a quantitative evaluation of this splitting in MWNT spectra.

The D' mode, the third resolved peak around $1620 \mathrm{~cm}^{-1}$, which is not present in the spectra of SWNT, is associated with the maximum in the graphene 2D phonon density of 
Chapter 1 Introduction to Carbon Nanotubes

state [87]. There are other reports which assign this peak to the disorder-induced scattering $[3,68]$.

The relative intensities $\left(\mathrm{I}_{\mathrm{D}} / \mathrm{I}_{\mathrm{G}}\right)$ have been employed to investigate the degree of perfection, or the degree of graphitication of MWNT samples. High intensity of the G mode over a weak or absent D mode indicates a high degree of crystallinity. Among reports [8, 68], as-grown MWNTs made by CVD hold a much higher $\mathrm{I}_{\mathrm{D}} / \mathrm{I}_{\mathrm{G}}$ ratio than MWNTs made by carbon arc method because the former tubes contain many more defects. In a uniformly distributed MWNTs sample, the ratio can be used to compare the impurity contents.

\subsection{Applications of carbon nanotubes}

Suggested uses of carbon nanotubes are always intriguing topics. Due to their nanometersize, uniform diameter and capillary structures, carbon nanotubes are perfect lightweight synthetic metals [32]. The applications and the properties and structures, on which applications were based, are listed in Table 1.3. Besides the intense interest in electronic applications as mentioned previously, there are still many research interests on applications, which are based on nanotubes' novel structures, and superb mechanical and electrical properties. These demonstrate that carbon nanotubes are not only intriguing objects for nanoscience but also very promising candidates for nanoengineering applications. 
Chapter 1 Introduction to Carbon Nanotubes

Table 1.3: Carbon nanotubes' applications and the properties on which they are based

\begin{tabular}{|c|c|}
\hline Applications & $\begin{array}{l}\text { The properties of CNTs on which the } \\
\text { applications are based }\end{array}$ \\
\hline $\begin{array}{l}\text { Conductive and high-strength composites } \\
{[71,94-96,111]}\end{array}$ & $\begin{array}{l}\text { Electrically and thermally conductive; } \\
\text { buckling strength }\end{array}$ \\
\hline $\begin{array}{l}\text { Field emission displays and radiation } \\
\text { sources }[36,44,55,99]\end{array}$ & Large ratio of length/diameter; conductive \\
\hline Hydrogen storage media $[15,21,97]$ & Capillary structure and large specific area \\
\hline $\begin{array}{l}\text { Energy storage and energy conversion devices } \\
{[2,14,38,72]}\end{array}$ & Large capacity and high specific area \\
\hline Nanometer-sized metal/alloy containers [7] & $\begin{array}{l}\text { Capillary structure, large ratio of surface } \\
\text { area/ weight and alloy of } \mathrm{C} \text { and metal }\end{array}$ \\
\hline $\begin{array}{l}\text { Nanometer-sized devices, transistors and } \\
\text { probes }[13,20,57,97]\end{array}$ & $\begin{array}{l}\text { Ballistic conduction, high current density } \\
\text { of } \mathrm{m}-\mathrm{CNT} \text {, high on-off ratio and gain of s- } \\
\text { CNT; robustness }\end{array}$ \\
\hline $\begin{array}{l}\text { Sensors* }[12,50,57,58,79,100,102,105, \\
110,114]^{*}\end{array}$ & $\begin{array}{l}\text { The alteration of the environment changes } \\
\text { the doping level on CNTs, swift response }\end{array}$ \\
\hline NEMS/MEMS $[23,33,35]^{* *}$ & $\begin{array}{l}\text { Unique structures, big buckling strength } \\
\text { and quick response to electric fields }\end{array}$ \\
\hline
\end{tabular}

Note:

* Based on nanometer-sized devices and transistors.

** NEMS/MEMS designates for nanometre-scale/micrometer-scale electrical mechanical system. 
Chapter 1 Introduction to Carbon Nanotubes

According to Richard Martel [74], two main reasons can be attributed for carbon nanotubes' intriguing electronic applications: One is, individual carbon nanotubes have the ability to carry electrical current at significantly higher densities than most metals and semiconductors. The charge carriers travel within the length of the nanotubes with high mobilities. The other is, carbon nanotubes are chemically inert and present no surface states. All the bonds in CNTs are engaged and there are no dangling bonds at the surface of the bond. This feature makes them more compatible with other materials such as oxides, and significantly reduces the need to passivise the oxide-semiconductor interface, as in the processing of silicon-based semiconductors. These mechanical and nanostructural aspects of potential applications are presented as follows.

\subsubsection{Nanotube-confined metal entity}

Attentions have been attracted onto application of nanotubes as nanometer-sized capillary containers for confining metal entities into small dimensions [7]. For example, Ga-filled carbon nanotube nanothermometre [45], was built by trapping a small piece of Ga inside a section of nanotube and calibrated by the 1D physical extension of the Ga nanorod against temperature. Then when the Ga/CNT nanothermometer is placed into the desired position, it will give the temperature in situ.

\subsubsection{Nanotube-based MEMS and NEMS}

Fabrication of nanotube-based micro-scale and nanometre-scale electro-mechanical devices [35], or CNT-based MEMS and NEMS is another interesting topic. A good example of CNT-based NEMS, an ultrasmall-sized rotational actuator [38], was built by A. Zettl, et al. on a section of a shortened MWNT. The microstructure of the NEMS was 
Chapter 1 Introduction to Carbon Nanotubes

fabricated by attaching an Au foil, which works as a flyer in the rotational system onto the outmost graphitic shell of a truncated section of a single MWNT. The outmost shell had been cut and only the inner shells were suspended on two anchor pads and served as the shaft in a motor. There is very small frictional force exerted between the inner and the outer shells of MWNTs. The Electric field was supplied by three Au pads, which were fabricated by lithographic method. This CNT-shafted micro-system was regarded as the smallest artificial electric motor so far.

Other CNT-based MEMS and NEMS have also been reported [25].

\subsubsection{AFM and STM probe tips}

Atomic force microscope (AFM) and scanning tunnelling microscope (STM) tips mounted with nanotubes can offer many advantages in nanoscale imaging [98]. Due to the characteristic geometry, structure, electronic properties and chemistry, the CNT tips have advantages in imaging structures with steep features, deep holes and softness [13], e.g. biological systems [109]. Enhanced resolution and high endurance [65] were found with CNT probes and rendered CNT tips to be used for nanolithography or as "nanopencils".

With CNT tips, neither the samples on which are tapped, nor the silicon probes are affected by the acts of tapping or touching, because the CNT tips will buckle to absorb the forces from the tapping or touching at contact. Bending $180^{\circ}$ without breaking beyond critical stress has been observed [22] on CNT tips.

The attachment of a CNT to a silicon tip can be achieved by either glue [22] or growing CNTs on the top of the tip [16] by CVD. 


\subsubsection{Energy storage device}

CNTs have been fabricated into energy-storage devices such as capacitors [41, 80] and batteries, in which CNTs can be used to replace the carbon black as the electrodes in these electrochemical devices. It has been reported $[2,80]$ that higher than $100 \mathrm{~F} / \mathrm{g}$ capacitance and a power density of $8 \mathrm{~kW} / \mathrm{kg}$ have been obtained. The reasons of the high capacitance can be attributed to the following: (1) the unique hollow structure of CNTs, which constructs a porous structure determined by the open spaces between entangled fibres; (2) large surface area of CNTs. It was reported by Niu et al. [80] that the specific surface area of as-grown CNTs can be as large as $430 \mathrm{~m}^{2} / \mathrm{g}$; (3) small distribution of the pore diameter, which contributes mostly to the capacitance. On the contrary, activated carbon, which is commonly adopted when making supercapacitors and has a high surface area of $2000-3000 \mathrm{~m}^{2} / \mathrm{g}$, can only offer a very small part of its theoretical capacitance. In addition, low equivalent series resistance and good cycling stability [14] have been found.

\subsubsection{CNT/polymer composites}

Carbon nanotubes are reported to be able to undertake the backbone role inside polymeric composites with improved mechanical resistance and electrical properties. Products of polymeric composites [95, 96] and fibres [24] containing carbon nanotubes were estimated to reach a market value of hundreds of millions pounds by 2009 [93]. Nanotubes have been used to replace carbon black in the applications of polymer/carbon material composite $[71,94-96,111]$ since CNTs possess nanometre-dimensions, light density and excellent comprehensive properties. Apart from possessing superb conducting property as mentioned previously, CNTs have a robust mechanical property. 
The elastic Young's modulus of CNTs approaches a value of $1.25 \times 10^{12} \mathrm{~Pa}$ [108] and another interesting aspect of CNTs' mechanical property is that the deformation of CNTs under mechanical load is elastic and it disappears after the load is released. The threshold ratio, which means the lowest percentage of loading of nanotubes inside the composite to conduct inside the whole polymer composite matrix, can be as low as $0.005 \mathrm{wt} \%$, which is of magnitudes lower than that of carbon blacks in composite polymer. This low threshold is of great significance for aerospace and automobile applications.

Coagulation-based carbon nanotube/polymer has been spun into fibres [24] and woven into textiles. The tensile strength of the composite fibres was reported to reach $1.8 \mathrm{Gpa}$, which matches that of spider silks, the toughest fibre in nature. And the energy-to-break reaches $570 \mathrm{Jg}^{-1}$, which is higher than the value of a spider silk. The woven textiles have their potential applications in distributed sensors, electronic interconnects, electromagnetic shields, antennas and batteries.

In addition, the idea of combining CNTs with conducting polymers, which has drawn lots of attentions since the discovery of CNTs, is also a very intriguing aspect of CNTs/polymeric composites [1]. CNT/conducting polymer composites have been long investigated with the hope of delivering CNT properties to a processable and synergetic host [113]. The electronic interaction between conjugated polymers and carbon nanotubes stabilizes the resulted composite and yields more sophisticated properties than the two materials alone. For example, inside the CNT/polyaniline matrix, CNTs are charge carrier acceptors while polyaniline is a good carrier donor. The interaction between CNTs and polyaniline increases the electron delocalisation. CNTs exhibit a doping effect on conducting polymers and function as conducting bridges between conducting domains 
Chapter I Introduction to Carbon Nanotubes

inside polymers. Therefore, conductivities of $\mathrm{CNT} /$ conducting polymer composite were increased by magnitude $[66,113]$.

In another example, a composite LED of polyphenylenevinylene (PPV) and MWNTs showed lifetimes in air up to 5 times longer than LED's without MWNTs. This interesting effect on lifetimes was attributed to the electronic interactions between PPV and MWNTs.

Above all, CNTs are a group of novel materials, which possess unique structures, intriguing properties and promising application prospects. However, before a full exploitation of their superb properties, there are still some topics, on which require addressing. Some technical hurdles in processing of CNTs, which will be addressed in the later chapters of this thesis, for example, finding techniques for purification and manipulation of CNTs, still remains a challenge.

The experimental work covered by this thesis involves two parts: Part I, synthesis of MWNTs by chemical vapour deposition, the aim of this part is to deliver a cheap and reliable synthetic route for MWNTs, to investigate the synthesis of MWNTs, including aligned and random MWNTs and to give an understanding of the CVD growth mechanism of aligned MWNTs and characterisations on morphology of CNTs we made; Part II, alignment and purification of multi-walled carbon nanotubes by dielectrophoresis. The aim of this part is to find a way for the above purposes. 


\section{Reference:}

1. H. Ago, M. S. P. Shaffer, D. S. Ginger, A. H. WIndle, and R. H. Friend (2000), Physical Review B, 61: p. 2286-2290.

2. K. H. An, W. S. Kim, Y. S. Park, Y. C. Choi, S. M. Lee, D. C. Chung, D. J. Bae, S. C. Lim, and Y. H. Lee (2003), Advanced Materials, 13: p. 497-500.

3. Y. Ando, X. Zhao, and H. Shimoyama (1999), Crystal Research and Technology, 34: p. 597-603.

4. S. M. Bachilo, M. S. Strano, C. Kittrell, R. H. Hauge, R. E. Smalley, and R. B. Weisman (2002), Science, 298: p. 2361-2166.

5. A. Bachtold, P. Hadley, T. Nakanishi, and C. Dekker (2001), Science, 294: p. 1317-1320.

6. A. Bachtold, C. Strunk, J.-P. Salvetat, J.-M. Bonard, L. Forro, T. Nussbaumer, and C. Schonenberger (1999), Nature, 397: p. 673-238.

7. J. Bao, Z. Xu, Z. Suo, Q. Zhou, and J. Hong (2002), Advanced Materials, 20: p. 1483-1486.

8. J. M. Benoit, J. P. Buisson, O. Chauvet, C. Godon, and S. Lefrant (2002), Physical Review B, 66: p. 073417.

9. M. Bockrath, D. H. Cobden, P. L. McEuen, N. G. Chopra, A. Zettle, A. Thess, and R. E. Smalley (1997), Science, 275: p. 1922-1925.

10. M. Bockrath, J. Hone, A. Zettl, P. L. McEuen, A. G. Rinzler, and R. E. Smalley (2000), Physical Review B, 61: p. R10606-R10608.

11. K. Bradley, J.-C. P. Gabriel, M. Briman, A. Star, and G. Gruner (2003), Physical Review Letters, 91: p. 218301-2.

12. K. Bradley, J.-C. P. Gabriel, M. Briman, A. Star, and G. Gruner (2003), Applied Physics Letters, 83: p. 3821-3823.

13. Y. C. Chang, D. C. Wang, C. S. Chang, and T. T. Tsong (2003), Applied Physics Letters, 82: p. 3541-3543.

14. Q.-L. Chen, K.-H. Xue, W. Shen, F.-F. Tao, S.-Y. Yin, and W. Xu (2004), Electrochimica Acta, 49: p. 4157-4161. 
15. Y. Chen, D. T. Shaw, X. D. Bai, E. G. Wang, C. Lund, W. M. Lu, and D. D. L. Chung (2001), Applied Physics Letters, 78: p. 2128-2130.

16. C. L. Cheung, J. H. Hafner, T. W. Odom, K. Kim, and C. M. Lieber (2000), Applied Physics Letters, 76: p. 3136-3138.

17. W. B. Choi, S. Chae, E. Bae, J.-W. Lee, B.-H. Cheong, J.-R. Kim, and J.-J. Kim (2003), Applied Physics Letters, 82: p. 275-277.

18. L. Ci, Z. Zhou, L. Song, X. Yan, D. Liu, H. Yuan, Y. Gao, J. Wang, L. Liu, W. Zhou, G. Wang, and S. Xie (2003), Applied Physics Letters, 82: p. 3098-3100.

19. P. G. Collins, K. Bradley, M. Ishigami, and A. Zettl (2000), Science, 287: p. 1801-1804.

20. J. B. Cui, R. Sordan, M. Burghard, and K. Kern (2002), Applied Physics Letters, 81: p. $3260-3262$.

21. R. Dagani, Temperest in a Tiny Tube, in Chemical \& Engineering News. 2002. p. 25-28

22. H. Dai, N. Franklin, and J. Han (1998), Applied Physics Letters, 73: p. 1508-1510.

23. H. Dai, E. W. Wong, and C. M. Lieber (1996), Science, 272: p. 523-526.

24. A. B. Dalton, S. Collins, E. Munoz, J. M. Razal, V. H. Ebron, J. P. Ferraris, J. N. Coleman, B. G. Kim, and R. H. Baughman (2003), Nature, 423: p. 703.

25. C. Day (2002), Physics Today, 16: p. 16-18.

26. C. Dekker (1999), Physics Today: p. 22-27.

27. V. Derycke, R. Martel, J. Appenzeller, and P. Avouris (2001), Nano Letters, 1: p. 453-456.

28. V. Derycke, R. Martel, J. Appenzeller, and P. Avouris (2002), Applied Physics Letters, 80: p. 2772-2775.

29. M. S. Dresselhaus, G. Dresselhaus, and P. C. Eklund (1996), in book. Science of Fullerences and Carbon Nanotubes San Diego: Elsevier Science

30. M. S. Dresselhaus, G. Dresselhaus, and P. C. Eklund., University of Pennsylvania Workshop. 1991.

31. M. S. Dresselhaus, G. Dresselhaus, A. Jorio, A. G. S. Filho, and R. Saito (2002), Carbon, 40: p. 2043-2061. 
32. T. W. Ebbesen (1997), in book. Carbon Nanotubes: Preparation and Properties Boca Raton, New York, London, Tokyo: CRC Press

33. T. W. Ebbesen, H. J. Jezec, H. Huiura, J. W. Bennett, H. F. Ghaemi, and T. Thio (1996), Nature, 382: p. 54-56.

34. M. Endo, H. Muramatsu, T. Hayashi, Y. A. Kim, M. Terrones, and M. S. Dresselhaus (2005), Nature, 433: p. 476.

35. M. R. Falvo, R. M. T. II, A. Helser, V. Chi, F. P. B. Jr, S. Washburn, and R. Superfine (1999), Nature, 397: p. 236-238.

36. S. Fan, M. G. Chapline, N. R. Franklin, T. W. Tomler, A. M. Casell, and H. Dai (1999), Science, 283: p. 512-514.

37. S. L. Fang, A. M. Rao, P. C. Eklund, P. Nikolaev, A. G. RInzler, and R. E. Smalley (1998), Journal of Material Research, 13: p. 2405-2411.

38. A. M. Fennimore, T. D. Yuzvinsky, W.-Q. Han, M. S. Funrer, J. Cumings, and A. Zettl (2003), Nature, 424: p. 408-410.

39. D. K. Ferry and J. P. Bird (2001), in book. Electronic Materials and Devices San Diego: Academic Press

40. J. E. Fischer, H. Dai, A. Thess, R. Lee, N. M. Hanjani, D. L. Dehaas, and R. E. Smalley (1997), Physical Review B, 55: p. R4921-R4924.

41. E. Frackowiak, K. Metenier, V. Bertagna, and F. Beguin (2000), Applied Physics Letters, 77: p. 2421-2423.

42. N. R. Franklin, Q. Wang, T. W. Tombler, A. Javey, M. Shim, and H. Dai (2002), Applied Physics Letters, 81: p. 913-915.

43. M. Freitag, M. Radosavljevic, Y. Zhou, A. T. Johnsona, and W. F. Smith (2001), Applied Physics Letters, 79: p. 3326-3328.

44. B. Gao, Guozhen, Z.Yue, Q. Qiu, Y. Cheng, H. Shimoda, L. Fleming, and O. Zhou (2001), Advanced Materials, 13: p. 1770-1773.

45. Y. Gao, Y. Bando, Z. Liu, and D. Golberg (2003), Applied Physics Letters, 83: p. 2913-2915.

46. J. Guo, M. Lundstrom, and S. Datta (2002), Applied Physics Letters, 80: p. $3192-$ 3194. 
47. N. Hamada, S. I. Sawada, and A. Oshiyama (1992), Physics Review Letters: p. 1579-1581.

48. A. Hassanien, M. Tokumoto, P. Umek, D. Mihailovic, and A. Mrzel (2001), Applied Physics Letters, 78: p. 808-810.

49. S. Iijima (1991), Nature, 354: p. 56-58.

50. Y.-T. Jang, S.-I. Moona, J.-H. Ahnb, Y.-H. Lee, and B.-K. Ju (2003), Sensors and Actuators B, 99: p. 118-122.

51. A. Javey, M. Shim, and H. Dai (2002), Applied Physics Letters, 80: p. 1064-1066.

52. A. Jorio, G. Dresselhaus, M. S. Dresselhaus, M. Souza, M. S. S. Dantas, M. A. Pimenta, A. M. Rao, R. Saito, C. Liu, and H. M. Cheng (2000), Physical Review Letters, 85: p. 2617-2620.

53. A. Jorio, R. Saito, J. H. Hafner, C. M. Lieber, M. Hunter, T. McClure, G. Dresselhaus, and M. S. Dresselhaus (2001), Physical Review Letters, 86: p. 1118 1121.

54. K. Keren, R. S. Berman, E. Buchstab, U. Srivan, and E. Braun (2003), Science, 302: p. 1380-138.

55. K. Kim, S. H. Lee, W. Yi, J. Kim, J. W. Choi, Y. Park, and J.-I. Jin (2003), Advanced Materials, 15: p. 1618-1622.

56. J. Kong, J. Cao, and H. Dai (2002), Applied Physics Letters, 80: p. 73-75.

57. J. Kong, M. G. Chapline, and H. Dai (2001), Advanced Materials, 13: p. 13841386.

58. J. Kong, N. R. Franklin, C. Zhou, M. G. Chapline, S. Peng, K. Cho, and H. Dai (2000), Science, 287: p. 622-625.

59. J. Kong, E. Yenilmez, T. W. Tombler, W. Kim, H. Dai, R. B. Laughlin, L. Liu, C. S. Jayanthi, and S. Y. Wu (2001), Physical Review Letters, 87: p. 106801-1.

60. J. Kong, C. Zhou, E. Yenilmez, and H. Dai (2000), Applied Physics Letters, 77: p. 3877-3979.

61. H. W. Kroto, J. R. Heath, S. C. O'Brien, R. F. Curl, and R. E. Samlley (1985), Nature: p. 162-163.

62. M. Kruger, M. R. Buitelaar, T. Nussbaumer, C. Schonenberger, and L. Forro (2001), Applied Physics Letters, 78: p. 1291-1293. 
63. H. Kuzmany, W. Plank, M. Hulman, C. Kramberger, A. Gruneis, T. Pichler, H. Peterlik, H. Kataura, and Y. Achiba (2001), European Physics Journal B, 22: p. 307-320.

64. L. Langer, V. Bayot, E. Grivei, P. I. J, J. P. Heremans, C. H. Olk, L. Stockman, C. V. Haesendonck, and Y. Buynseraede (1996), Physical Review Letters, 76: p. 479-482.

65. T. Larsen, K. Moloni, M. A. Eriksson, M. G. Lagally, and C. T. Black (2002), Applied Physics Letters, 80: p. 1996-1998.

66. M. Lefenfeld, G. Blanchet, and J. A. Rogers (2003), Advanced Materials, 15: p. 1188-1191.

67. S. G. Lemay, J. W. Jassenwenhoven, M. v. d. Hout, M. Mooij, M. J. Bronikowski, P. A. Willis, R. E. Smalley, L. P. Kou, and C. Dekker (2001), Nature, 412: p. $617-$ 620.

68. W. Li, H. Zhang, C. Wang, Y. Zhang, L. Xu, K. Zhu, and S. Xie (1997), Applied Physics Letters, 70: p. 2684-2686.

69. Y. X. Liang, Q. H. Li, and T. H. Wang (2004), Applied Physics Letters, 84: p. 3379-3381.

70. S. C. Lim, Y. C. Choi, H. J. Jeong, Y. M. Shin, K. H. An, D. J. Bae, Y. H. Lee, N. S. Lee, and J. M. Kim (2001), Advanced Materials, 13: p. 1563-1567.

71. Y. Lin, B. Zhou, K. A. S. Fernando, P. Liu, L. F. Allard, and Y.-P. Sun (2003), Macromolecules, 36: p. 7199-7204.

72. X. Liu, C. Lee, C. Zhou, and J. Han (2001), Applied Physics Letters, 79: p. 3329 3331 .

73. I. Loa (2003), Journal of Raman Spectroscopy, 34: p. 611-627.

74. R. Martel (2002), Nature Materials, 1: p. 203-204.

75. R. Martel, T. Schimidt, H. R. Shea, T. Hertel, and P. Avouris (1998), Applied Physics Letters, 73: p. 2447-2449.

76. P. L. McEuen, M. Fuuhrer, and H. Park (2003), IEEE transactions on nanotechnology, 1: p. 78-87.

77. J. W. Mintmire, B. I. Dunlap, and C. T. White (1992), Physical Review Letters, 68 : p. 631-634. 
Chapter 1 Introduction to Carbon Nanotubes

78. J. W. Mintmire and C. T. White (1998), Physical Review Letters, 81: p. 25062509.

79. T. Modl, N. Koratkar, E. Lass, B. Wei, and P. M. Ajayan (2003), Nature, 424: p. 171-174.

80. C. Niu, E. K. Sichel, R. Hoch, D. Moy, and H. Tennent (1997), Applied Physics Letters, 70: p. 1480-1482.

81. T. W. Odom, J.-1. Huang, C. L. Cheung, and C. M. Lieber (2000), Science, 290: p. 1549-1552.

82. T. W. Odom, J.-1. Huang, P. Kim, and C. M. Lieber (1998), Nature, 391: p. 62-64.

83. M. A. Pimenta, A. Marucci, S. A. Empedocles, M. G. Bawendi, E. B. Hanlon, A. M. Rao, P. C. Eklund, R. E. Smalley, G. Dresselhaus, and M. S. Dresselhaus (1998), Rapid Communications, Physical Review B, 58: p. R 16 016- R 16019.

84. P. Poncharal, S. Frank, Z.L.Wang, and W. A. d. Heer (1999), Europe Physics Journal D, 9: p. $77-79$.

85. V. N. Popov (2004), Material Science and Engineering, 43: p. 61-102.

86. C. Portet, P. L. Taberna, P. Simon, and E. Flahaut (2005), Journal of Power Sources, 139: p. 371-378.

87. A. M. Rao, A. Jorio, M. A. Primenta, M. S. S. Dantas, R. Saito, G. Dresselhaus, and M. S. Dresselhaus (2000), Physical Review Letters, 84: p. 1820-1823.

88. A. M. Rao, E. Richter, S. Bandow, B. Chase, P. C. Eklund, K. A. Williams, S. Fang, K. R. Subbaswamy, M. Mendon, A. Thess, R. E. Smalle, G. Dresselhaus, and M. S. Dresselhaus (1997), Science, 275: p. 187-191.

89. S. Reich and C. Thomsen (2000), Physical Review Letters, 85: p. 3544.

90. T. Rueches, K. Kim, E. Joselevich, G. Y. Tseng, C.-L. Cheung, and C. M. Lieber (2000), Science, 289: p. 94-97.

91. R. Saito, G. Dresselhaus, and M. S. Dresselhaus (1998), in book. Physical Properties of Carbon Nanotubes London: Imperial College Press

92. R. Saito, M. Fukita, G. Dresselhaus, and M. S. Dresselhaus (1992), Applied Physics Letters: p. 2204-2206. 
Chapter 1 Introduction to Carbon Nanotubes

93. J. K. W. Sandler, J. E. Kirk, I. A. Kinloch, M. S. P.Shaffer, and A. H. Windle (2003), Polymer, 44: p. 5893-5899.

94. J. K. W. Sandler, S. Pegal, M. Cadek, F. Gojny, M. v. Es, J. Lohmar, W. J. Blau, K. Schulte, A. H. Windle, and M. S. P. Shaffer (2004), Polymer, 45: p. 2001-2015.

95. J. K. W. Sandler, M. S. P. Shaffer, T. Prasse, W. Bauhofer, K. Schulte, and A. H. Windle (1999), Polymer, 40: p. 5967-5971.

96. L. S. Schadler, S. C. Gianaris, and P. M. Ajayan (1998), Applied Physics Letters, 73: p. $3842-3844$.

97. J. M. Skowronski, P. Scharff, N. Pfander, and S. Cui (2003), Advanced Materials, 15: p. 55-57.

98. E. S. Snow, P. M. Campbell, and J. P. Novak (2002), Applied Physics Letters, 80: p. 2002-2004.

99. J. I. Sohn, S. Lee, Y.-H. Song, S.-Y. Choi, K.-I. Cho, and K.-S. Nam (2001), Current Applied Physics, 1: p. 61-65.

100. J. R. Stetter, K. Bradley, J. Cumings, J.-C. Gabriel, G. Gruner, and A. Star. Nanotech 2003, p. 313-316, 2003. San Francisco, CA, U.S.A.: Computational Publications.

101. J. A. Stroscio and R. M. Feenstra (1993), in book. Scanning Tunneling Microscopy New York: Academic

102. J. Suehiro, G. Zhou, and M. Hara (2003), Journal of Physcis D: Applied Physics, 36: p. L109-L114.

103. S. J. Tans, A. R. M. Verschueren, and C. Dekker (1998), Nature, 393: p. 49-52.

104. L. Valentini, C. Cantalini, I. Armentano, J. M. Kenny, L. Lozzi, and S. Santucci (2003), Journal of Vacuum Science and Technology B, 21: p. 1996-2000.

105. O. K. Varghese, P. D. Kichambre, D. Gong, K. G. Ong, E. C. Dickey, and C. A. Grimes (2003), Sensors and Actuators B, 81: p. 32-41.

106. J. W. G. Wildoer, L. C. Venema, A. G. RInzler, R. E. Smalley, and C. Dekker (1998), Nature, 391: p. 59-64.

107. S. J. Wind, J. Appenzeller, R. Martel, V. Derycke, and P. Avouris (2002), Applied Physics Letters, 80: p. 3817-3819.

108. E. W. Wong, P. E. Sheehan, and C. Lieber (1997), Science, 277: p. 1971-1975. 
Chapter 1 Introduction to Carbon Nanotubes

109. S. S. Wong, J. D. Harper, J. Peter T. Lansbury, and C. M. Lieber (603), Journal of American Chemistry Society, 120: p. 603-704.

110. Y. M. Wong, W. P. Kang, J. L. Davidson, A. Wisitsorat, and K. L. Soh (2003), Sensors and Actuators B, 93: p. 327-332.

111. X. Xu, M. M. Thwe, C. Shearwood, and K. Liao (2002), Applied Physics Letters, 81: p. 2833-2835.

112. S. Yan (2000), in book. Fundamentals of Solid State Physics. The serie of advanced physics of Peking University, ed. Congshou Gao Beijing: Peiking University Press

113. H. Zengin, W. Zhou, J. Jin, R. Czerw, J. Dennis W. Smith, L. Echegoyen, D. L. Carroll, S. H. Foulger, and J. Ballato (2002), Advanced Materials, 14: p. 14801483.

114. Q. Zhao, J. R. Wood, and H. D. Wagner (2001), Applied Physics Letters, 78: p. 1748-1750.

115. C. Zhou, J. Kong, E. Yenilmez, and H. Dai (2000), Science, 290: p. 1552-1555. 


\section{Part I}

Synthesis of Carbon Nanotubes by Chemical Vapour Deposition 


\section{Chapter 2}

\section{Synthesis of Carbon Nanotubes}

The previous chapter reviewed the background of the structures and properties of carbon nanotubes. A further review on the synthesis of carbon nanotubes will be given in this chapter. A brief history of three basic synthetic methods, carbon arc method, laser ablation method and chemical vapour deposition method, will be included. More details of the chemical vapour deposition method will be presented here than the other two methods, and its two proposed growth mechanisms, tip growth and base growth will also be discussed.

\subsection{Development}

Since the discovery of carbon nanotube [33], three major different synthetic methods, carbon arc, laser ablation and chemical vapour deposition methods with innovative experimental setups have been investigated to meet the demands of reliably and repeatably producing CNTs.

\subsubsection{Carbon arc discharge method}

The batch of multi-walled carbon nanotubes which was discovered by Ijima, was prepared by a carbon arc discharge evaporation method similar to that used for the synthesis of fullerene samples [42]. This method had also been adopted in the manufacture of carbon whiskers [3]. 
In this method, a huge direct current (200 A, $20 \mathrm{~V}$, as reported by Ebbesen [19]) was applied to generate a high-energy plasma between two electrodes (see Figure 2-1). The cathode is the deposit electrode, which collects soot and the anode is a movable carbon rod, which is consumed in the arc. The apparatus is attached to a vacuum pump and an inert gas supply, usually helium.

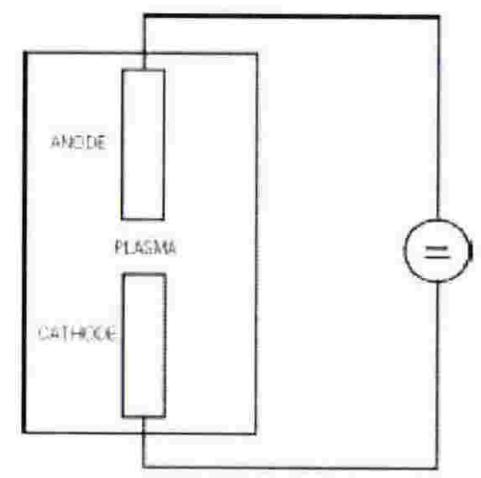

Figure 2-1 Scheme of a carbon arc discharge apparatus used by Iijima [33].

A typical carbon arc process takes place as follows: Once the pressure of the inert gas inside the reaction chamber is stabilized, the power supply is turned on. The distance between the two electrodes is adjusted until an arc is struck and plasma forms. The temperature inside the chamber could reach up to $3700{ }^{\circ} \mathrm{C}$. The carbon on the anode is consumed while samples are deposited on the cathode.

Later on, large-scale synthesis of multi-walled and single-walled carbon nanotubes by a variant of the standard arc-discharge technique were independently reported by Ebbesen et al. [19] and Journet et al. [37].

The carbon arc method for producing CNTs is simple and easy to operate, however, to obtain CNTs with high yields and purities, a careful control over the experimental 
Chapter 2 Synthesis of Carbon Nanotubes

conditions is required, otherwise, impurities, such as liquid carbon, carbon-glass bead and carbon nanoparticles [27] will be the main products in the samples.

\subsubsection{Laser ablation method}

A laser ablation technique was first adopted by R. Smalley et al. [61] in 1996 to synthesize single-walled nanotubes at a low percentage yield. Later on, the yield was increased to $80 \%$. In this method, a laser beam was applied to a carbon target in order to vaporize a mixture of graphite and metal catalyst $(\mathrm{Co}, \mathrm{Ni})$. The target was contained within a horizontal tube with a flow of inert gas. And this tube was placed within a tube furnace at $1200{ }^{\circ} \mathrm{C}$. The nanotubes are harvested on a water-cooled copper collector outside the furnace from evaporated graphite (see Figure 2-2).

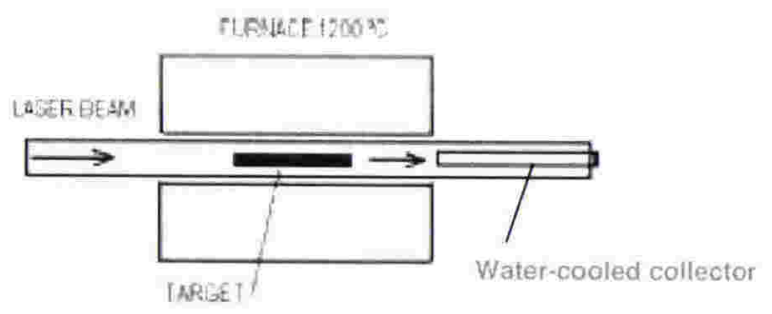

Figure 2-2 Scheme of a laser-ablation apparatus for the synthesis of carbon nanotubes.

\subsubsection{Chemical vapour deposition}

The two methods mentioned above lack the ability to be scaled-up. Another disadvantage of the two methods is that the synthesis temperatures are too high and therefore they consume huge energy and are not easy to be properly controlled. Chemical Vapour Deposition (CVD), the method which had been universally used to deposit metals on $\mathrm{Si}$ substrates in the semiconductor industry [24], was adopted to overcome some of the 
Chapter 2 Synthesis of Carbon Nanotubes

problems with the laser-ablation and arc-discharge methods. Nowadays, a plant to manufacture carbon nanotubes by CVD with an output hundreds of tons a year is under way [54]. The CVD setup will be detailed in the next section.

\subsection{Chemical vapour deposition}

CVD works as follows: a substrate is placed in a reaction chamber with an inert gas and heated to an elevated temperature. Hydrocarbon gases are then introduced into the hot reaction chamber at a specified flow rate, and come into contact with catalysts on the substrate surface. As reactants react with the catalysts, CNTs are grown on the substrate. CVD is operated at much lower temperature (usually less than $1000{ }^{\circ} \mathrm{C}$ ) and yields much more products than the two earlier methods. However, due to the low growth temperature, nanotubes prepared by CVD have more defects and curves than its two counterparts. This dilemma remains a challenge for CVD in the attempt to produce large-quantity of defectfree CNTs.

There are several different reported methods of CVD.

\section{Thermal CVD}

In a thermal CVD $[31,46,73]$, the catalyst is derived from a sublimable precursor or a precursor which has been pre-deposited onto the growth substrate and then carbon source is decomposed in a pyrolysis furnace, forming carbon nanotubes.

\section{Hot-filament CVD}

The key characteristic of a hot-filament CVD (which in some references is referred to as hot-wire CVD) $[17,35,50]$ is that, a tungsten filament, with big current flow, operating at a temperature around $1000{ }^{\circ} \mathrm{C}$, is placed inside the reaction chamber in order to crack 
Chapter 2 Synthesis of Carbon Nanotubes

the carbon source into carbon species, and to heat the growth substrate (where growth temperature is lower than $700^{\circ} \mathrm{C}$ ) which is set above the filament.

\section{Plasma-enhanced CVD}

In a plasma-enhanced CVD $[7,8,12,16,32,62]$, the catalyst forms a thin layer on the top of the growth substrate beforehand. In a typical process, plasma enhances the process of dissociating the hydrocarbon into carbon species and therefore increases carbon supplies for the growth of CNTs. Compared with the two CVD methods mentioned above, plasma-enhanced CVD yields higher growth rate and better alignment. However, individual CNTs, which are grown by plasma CVD, contain more contents of defects in their sidewalls and are of poor quality because of the low growth temperature and the rapid growth rate.

\subsubsection{Choice of catalyst}

The $3 d$ transition metals, $\mathrm{Fe}, \mathrm{Co}$ and $\mathrm{Ni}$ are essential $[14,31]$ for the synthesis of carbon nanotubes by the catalytic pyrolysis of hydrocarbons. The metals can be used separately or combined to give composite catalysts. In a Fe, Co or Ni-catalysed growth of CNTs, the catalysts dissolve carbon species from the atmosphere in the reaction chamber and then the dissolved carbons precipitate from the catalysts and form into tubules.

Studies have revealed that the size of the catalyst particles [31] and the reaction temperature [46] are the two key factors which affect the morphology of carbon nanotubes and the kinetics of the growth reaction. The size of the catalyst particles determines the diameter of the nanotubes, therefore, in order to obtain CNTs with narrowly-distributed diameters, uniform sizes of the catalysts are essential. And an elevated reaction temperature drastically increases the rate at which carbon atoms 
dissolve and diffuse into the catalysts, and therefore determines the formation kinetics of CNTs.

It has been proven by Bonard et al. [40] that $\mathrm{Fe}$ is most efficient among the three catalyst metals in solutions, in terms of yield, growth rate and quality of tubes in a thermal CVD method for multi-walled nanotube synthesis. In their paper [40], the authors attributed the better performance of $\mathrm{Fe}$ than $\mathrm{Ni}$ and $\mathrm{Co}$ to the fact that a gel-like film was only formed out of $\mathrm{Fe}_{2} \mathrm{O}_{3}$ but not with the other two catalysts.

Also, recent reports demonstrated that long strands of nanotube ropes with lengths in centimetres $[48,76]$ were successfully made from Fe-containing catalysts only by a floating bed thermal CVD.

And more, iron is more accessible and easier to handle for a cheap production of carbon nanotubes than the other two catalysts and in addition, there is good affinity between iron and carbon under the thermal CVD condition. All these merits made Fe the best choice for us to make MWNTs by thermal CVD. In the next chapter, we choose $\mathrm{Fe}\left(\mathrm{NO}_{3}\right)_{3}$ as the catalyst for growing of multiwalled nanotubes. Also, we use two Fe-containing precursors, ferrocene and iron (II) phthalocyanine (FePC) to grow aligned multi-walled carbon nanotubes (MWNTs) vertically on $\mathrm{SiO}_{2}$ and $\mathrm{Si}_{3} \mathrm{~N}_{4}$ substrates in our experiment.

\subsubsection{Growth mechanism}

The growth of carbon nanotubes by CVD involves four steps. Firstly, an ultrathin catalyst film forms into clusters on the surface of the substrate after being derived from the precursor or being deposited by evaporation. Upon growth temperature, the metal melts and turns into a liquid. Secondly, hydrocarbon is decomposed into carbon species, and 
then the carbon atoms dissolve in metal liquids to form metal-carbon alloys. Thirdly, the carbon atoms migrate, over-saturate in the transition metals and diffuse to the surface of the catalyst particles. Finally, carbon species assemble into tubular forms.

Two different mechanisms have been proposed on how carbon species form into tubules after they diffuse from the nucleation sites. One is bottom growth mechanism [7, 43, 44, 55], as depicted in Figure 2-3.

(a)

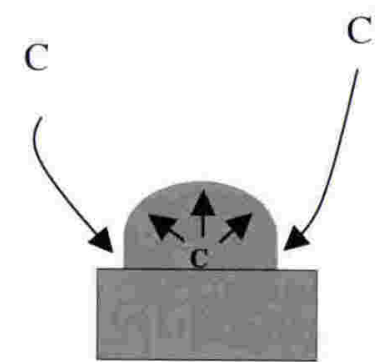

(b)
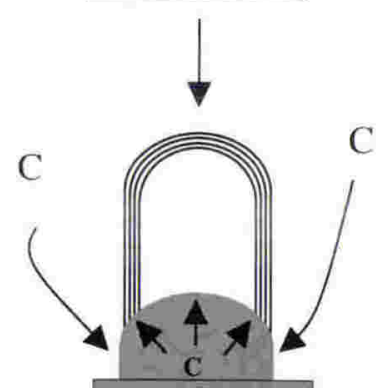

C
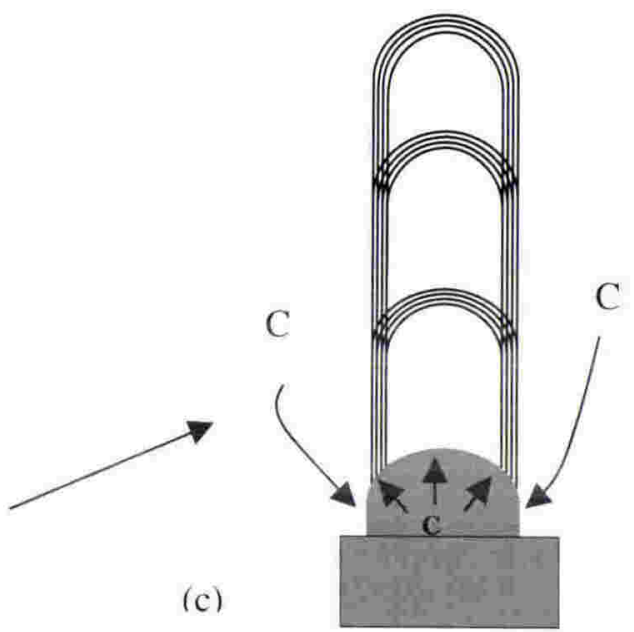

Figure 2-3 Schematic diagram of the base growth model [43]. The solid straight arrows in the diagram indicate the time sequence. (a) Hydrocarbon is decomposed into carbon species, which diffuse into nucleation site on growth substrate. (b) Carbon species continue diffusing into the site, over-saturate in the metal-carbon alloy and form into an encapsulated tubule, and (c) More carbon species continue to diffuse in the catalytic site, form into encapsulated tubules from the site and push the previous tubular sections up.

Under this mechanism, catalyst metal plays the key role in the whole process. After carbon species diffuse through the surface into the bulk of the metal clusters, they form capped graphitic tubules upon the nucleation site. Then the carbon species from the continuous supply repeatedly diffuse into the catalytic site and join into the open edges of 
the caps to form more caps and then push the tubular sections upward. The tubular wall continues to grow till the carbon sources are depleted. This mechanism is based on the observation of bamboo-shaped nanotubes, which contain compartments inside the tube with curvatures pointing to the CNT tips.

(a)
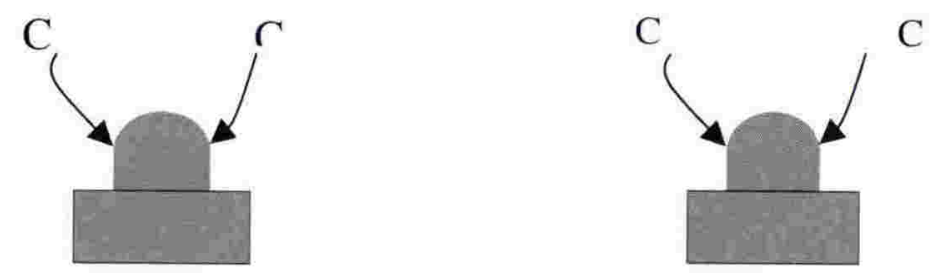

(b)
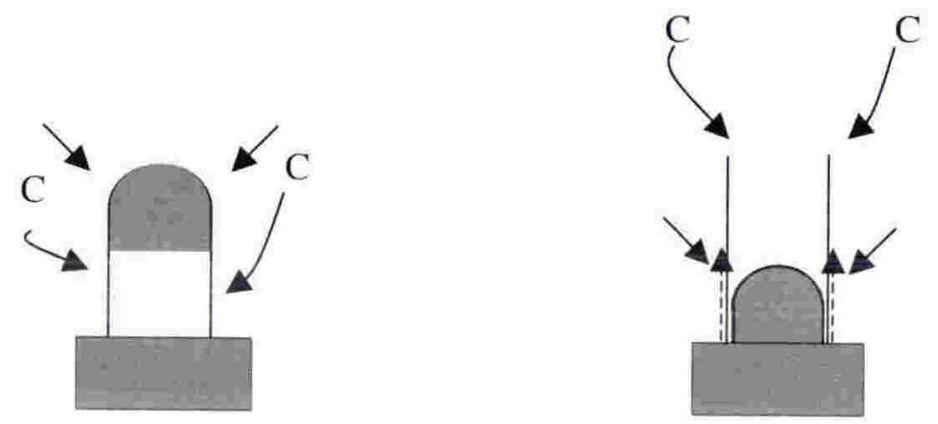

(c)
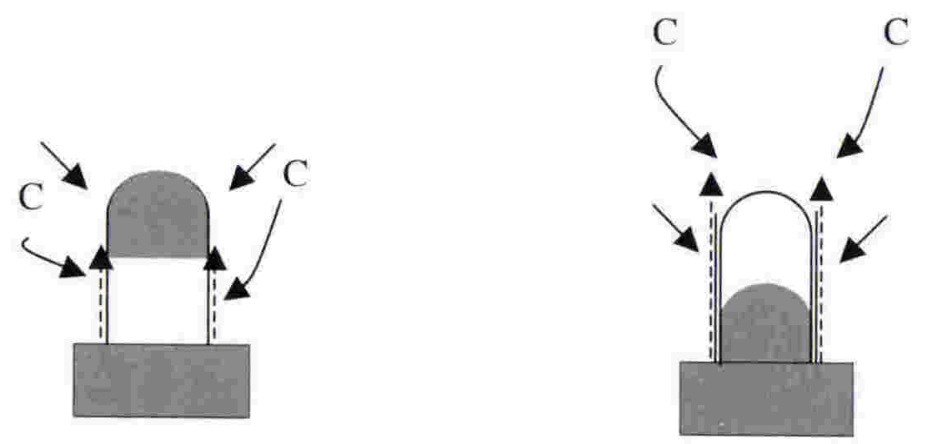

Case 1

Case 2

Figure 2-4 Depiction of the tip growth mechanism [49], the solid arrows and dashed arrows in the diagram indicate carbon species from the supply and the diffusion of carbon species during the growth of a CNT, respectively. In case 1, shown on the left-hand side, the diffusion time is much less than the saturation time, while in case 2, shown on the right-hand side, the former is much more than the latter. (a) In the initial stage, a metal particle is deposited on the top of the substrate, and then carbon atoms are absorbed on the metal particle, (b) the nucleation stage, and (c) the post-nucleation stage. 
Chapter 2 Synthesis of Carbon Nanotubes

The other mechanism is the tip growth model $[49,58]$. In the contrast to the base mechanism, which describes a carbon/metal nucleation site interaction process, the tip growth mechanism, as depicted in Figure 2-4, proposes that carbon species hit the nanotube surfaces in a chemisorption process and diffuse along the surfaces to feed the growth at nanotube tips.

Under this mechanism, whether the catalytic particle stays in the base or the tip depends on the competition between the carbon diffusion rate (this refers to carbon atoms diffusing in nanotube shells, rather than in metal clusters) and the carbon saturation rate. If diffusion is much faster than saturation, carbon species will drop on the bottom of the tube and keep lifting the enclosed metal tip off the substrate, as indicated in the Case 1 of Figure 2-4. If the latter one is much faster, carbon species will stay on the unclosed tip and the catalytic particle will stay on the base, as shown in Case 2 of Figure 2-4. The role that the catalyst metal particles play under the tip growth mechanism is that the size of particles will define the diameter of the nanotubes.

Both the base growth and the tip growth might exist at the same time or one might follow the other [1]. The reaction temperature impacts on the solubility of carbon species in the metal catalysts, the nucleation process and the diffusion rate. Different sources [29, 31, $46,59]$ reported that $750^{\circ} \mathrm{C}$ to $950^{\circ} \mathrm{C}$ are appropriate pyrolysis temperatures for thermal CVD using $\mathrm{Fe}$ as the catalyst and $\mathrm{C}_{2} \mathrm{H}_{2}$ as the carbon source.

\subsubsection{Role of hydrogen in the synthesis of carbon nanotubes}

Hydrogen has been found $[6,53]$ being able to efficiently promote the synthesis CNTs. It has been reported by Ruhmond et al. that [53], in a thermal CVD reaction which was 
engaged 20 minutes after the hydrogen supply had been cut off, the suspensions of hydrogen result in thickening sidewalls of MWNTs and unwanted products of carbon fibres with diameters of more than $1 \mu \mathrm{m}$. The "sidewall thickening effect" can be attributed to either an increasing concentration of carbon species in the reaction chamber or an accelerated precipitation rate of carbon species.
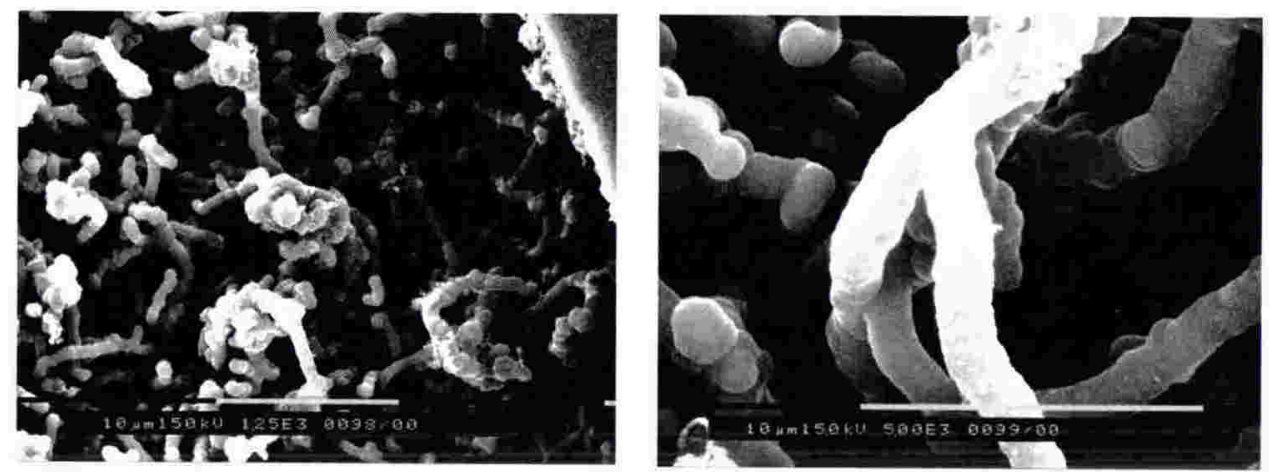

Figure 2-5 Unwanted carbon whiskers were obtained in the early attempts of synthesis carbon nanotubes from ferrocene precursor at $950{ }^{\circ} \mathrm{C}$ without hydrogen supply.

In the early stage of the experimental work of this thesis, we observed a similar phenomenon when we attempted to synthesize carbon nanotubes at $950{ }^{\circ} \mathrm{C}$ from the ferrocene precursor using $\mathrm{CH}_{4}$ as the carbon source with the absence of hydrogen. We found that, instead of obtaining CNTs, fibre-like carbon whiskers with diameters ranging from 1-2 $\mu \mathrm{m}$ were made, as shown in Figure 2-5.

These observations are associated to the disproportionation reaction of hydrocarbon, for example, the decomposition of $\mathrm{CH}_{4}$,

$$
\mathrm{CH}_{4} \rightarrow \mathrm{C} \bullet+4 \mathrm{H} \bullet
$$

In a hydrogen-participated synthesis of CNTs, hydrogen species, which are released from hydrogen gases, shift the equilibrium above to the left. Thus one of the roles which hydrogen plays in the synthesis of MWNTs, might be limiting the release of carbon 
species from the hydrocarbon. A total cut-off of hydrogen results in a rapid rate of hydrocarbon dissociation, therefore much more dense supply of carbon species leads to the formation of up to $2 \mu \mathrm{m}$-thick walls in carbon fibres.

These authors in the report [53] also proposed another role of hydrogen as reducing the metal catalyst particles so that the activity of these catalysts is maintained in the synthesis of CNTs.

However, Dai et al. [72] recently proposed that hydrogen plays a negative role in the synthesis of SWNTs after feeding oxygen into the reaction camber to remove the hydrogen radicals. The disproportionation reaction of hydrocarbon inevitably generates $\mathrm{H}$ species, which favour the establishment of $s p^{3}$ structures and unfavour $s p^{2}$ formation of SWNT structures.

As suggested by the same author [72], however, MWNTs should be more stable than SWNTs and therefore the negative effect of hydrogen should be less addressed in the growth of aligned MWNT. And the positive roles of hydrogen, which include reducing the metallic catalysts and restraining the hydrocarbon from oversupplying carbon species, are more predominant than its negative impacts in the synthesis of MWNTs.

\subsection{Alignment of carbon nanotubes}

Carbon nanotubes are favourable cathode materials in vacuum electron emission devices due to their small sizes, large aspect ratio and flexible structures. The applications of carbon nanotubes as the cathode materials in electron emitters [23, 26, 68, 71], large panel displays [25] and miniaturized gas sensors [52] have huge market potentials. Alignment of carbon nanotubes makes structures with large specific areas and is a very 
desirable factor for these applications. And also, aligned carbon nanotubes can be used for hydrogen storages [13] and mechanical enhancement [41].

By taking different methods, during- and post-synthesis alignment of CNTs can be achieved. The during-synthesis alignment methods are to be introduced here and a postsynthesis method will be given in Chapter 6 .

Aligned carbon nanotubes can be achieved from different precursors and synthetic routes. There are a number of reports showing a variety of precursors and their growth mechanisms for the synthesis of aligned nanotubes. For example:

1. Growing of vertically aligned CNTs from $\mathrm{Fe}$ - or $\mathrm{Ni}$ - derived catalysts on flat $\mathrm{SiO}_{2}$ substrates. These include iron pentacarbonyl- [53], FePC- [17, 28] and ferrocene-derived $[38,56]$ catalysts on silica glass, oxidized $\mathrm{Si}$ wafers [75] and Ni-deposited [51] $\mathrm{SiO}_{2}$ or $\mathrm{Si}$ substrates [65].

2. Depositing aligned CNTs from Fe-derived catalyst on porous substrates, such as mesoporous silica [47], or the pores of anodic aluminium oxide (AAO) $[34,36,45,68]$. Under a proper electrochemical setup [34], AAO yields structures with aligned nanometer-sized pores. Then carbon nanotubes can be grown inside the pores from predeposited catalysts, Aligned pure CNT structures can then be obtained by dissolving AAO afterward.

3. Achieving alignment of nanotubes by applying electric fields during the CVD process, for example, using electron beam evaporation [4] and Fe-patterned porous silicon [20, 59] by DC electric field-assisted thermal CVD, using an electric field-biased substrate [2]on $\mathrm{Ni}$ - or Co-deposited glass $[57,60,74]$, or using $\mathrm{Si}$ wafers $[8,15,67]$ or stainless steel 
wafers $[8,12]$ by plasma CVD. Both aligned SWNTs and MWNTs can be produced from these approaches.

\subsection{Micro-patterning of aligned carbon nanotubes}

Aligned carbon nanotubes can be patterned with micrometer resolutions for further uses in micro-electromechanical applications. These patterns can be achieved by pre-defining the catalysts onto templates by laser pulse etching $[5,60]$, conventional lithographic technique $[10,18,28,63,66]$, photo-resist patterning $[29,30,69,70]$, plasma patterning [11], micrometre-resolution masks [28, 64], microcontact-printing [22, 39], controlled site density [62] or micro-tweezers techniques [21]. Then aligned nanotubes are grown preferentially to inherit the template topography of their substrates, and being patterned into pre-determined microstructures.

By selectively inhibiting CNTs growth on some surfaces [9] with topographical masking of patterned substrates, aligned nanotubes can be grown into 3-dimensional structures for applications in electronic switching, memory storage, sensing and actuation. An experimental method of selectively growing CNTs is to be reported in the next chapter.

\section{Reference:}

1. S. Amelinckx, X. B. Zhang, D. Bernaerts, X. F. Zhang, V. Ivanov, and J. B. Nagy (1994), Science, 265: p. 635-639.

2. Y. Avigal and R. Kalish (2001), Applied Physics Letters, 78: p. 2291-2293.

3. R. Bacon (1960), Journal of Applied Physics, 31: p. 283-290. 
4. E. J. Bae, W. B. Choi, K. S. Jeong, J. U. Chu, G.-S. Park, S. Song, and I. K. Yoo (2002), Advanced Materials, 14: p. 277-279.

5. L. P. Biro, G. Molnar, I. Szabo, Z. Vertesy, Z. E. Horvath, J. Gyulai, Z. Konya, P. Piedigrosso, A. Fonseca, J. B. Nagy, and P. A. Thiry (2000), Applied Physics Letters, 76: p. 706-708.

6. K. Bladh, L. K. L. Falk, and F. Rohmund (2000), Applied Physics A: Materials Science \& Processing, 70: p. 317-322.

7. C. Bower, O. Zhou, W. Zhu, D. J. Werder, and S. Jin (2000), Applied Physics Letters, 77: p. 1767-2769.

8. C. Bower, W. Zhu, S. Jin, and O. Zhou (2000), Applied Physics Letters, 77: p. 830-832.

9. A. Cao, R. Baskaran, M. J. Frederick, K. Turner, P. Ajayan, and G. Ramanath (2003), Advanced Materials, 15: p. 1105-1109.

10. A. Cao, B. Wei, Y. Jung, R. Vajtai, P. M. Ajayan, and G. Ramanath (2002), Applied Physics Letters, 81: p. 1297-1299.

11. Q. Chen and L. Dai (2000), Applied Physics Letters, 76: p. 2719-2721.

12. Y. Chen, D. T. Shaw, X. D. Bai, E. G. Wang, C. Lund, W. M. Lu, and D. D. L. Chung (2001), Applied Physics Letters, 78: p. 2128-2130.

13. Y. C. Chen, N. R. Raravikar, L. S. Schadler, P. M. Ajayan, Y. P. Zhao, T. M. Lu, G. C. Wang, and X. C. Zhang (2002), Applied Physics Letters, 81: p. 975-977.

14. H. M. Cheng, G. S. F. Li, H. Y. Pan, L. L. He, X. Sun, and M. S. Dresselhaus (1998), Applied Physics Letters, 72: p. 3282-3284.

15. Y. C. Choi, Y. M. Shin, Y. H. Lee, B. S. Lee, G.-S. Park, W. B. Choi, N. S. Lee, and J. M. Kim (2000), Applied Physics Letters, 76: p. 2367-2369.

16. Y. C. Choi, Y. M. Shin, S. C. Lim, D. J. Bae, Y. H. Lee, B. s. Lee, and D.-C. Chung (2000), Journal of Applied Physics, 88: p. 4898-4903.

17. L. Dai and S. Huang (1999), Multilayer Carbon Nanotube Films, Australia Patent, Commonwealth Scientific and Industrial Research Organization, WO 00/63115

18. L. Dai and A. W. H. Mau (2001), Advanced Materials, 13: p. 899-913.

19. T. W. Ebbesen and P. Ajayan (1992), Nature, 358: p. 220-222. 
20. S. Fan, M. G. Chapline, N. R. Franklin, T. W. Tomler, A. M. Casell, and H. Dai (1999), Science, 283: p. 512-514.

21. B. Gao, Guozhen, Z.Yue, Q. Qiu, Y. Cheng, H. Shimoda, L. Fleming, and O. Zhou (2001), Advanced Materials, 13: p. 1770-1773.

22. G. Gu, G. Philipp, X. Wu, M. Burghard, A. M. Bittner, and S. Roth (2000), Advanced Functional Materials, 11: p. 295-298.

23. M. A. Guillorn, M. D. Hale, V. I. Merkulov, M. L. Simpson, G. Y. Eres, H. Cui, A. A. Puretzky, and D. B. Geohegan (2002), Applied Physics Letters, 81: p. 28602862.

24. C. A. Harper (2002), in book. Electronic Assembly Fabrication. Electronic Packaging and Interconnection Series New York: McGraw-Hill

25. W. A. d. Heer, J.-M. Bonard, K. Frauth, A. Chatelain, L. Forro, and D. Ugarte (1997), Advanced Materials, 9: p. 87-89.

26. W. A. d. Heer and A. Chatelain (1995), Science, 270: p. 1179-1180.

27. W. A. d. Heer, P. Poncharal, C. Berger, J. Gezo, Z. Song, J. Bettini, and D. Ugarte (2005), Science, 307: p. 907-910.

28. S. Huang, L. Dai, and A. W. H. Mau (1999), Journal of Physical Chemistry, 103: p. 4223-4227.

29. S. Huang, L. Dai, and A. W. H. Mau (2002), Advanced Materials, 14: p. 11401143 .

30. S. Huang, B. Maynor, X. Cai, and J. Liu (2003), Advanced Materials, 15: p. 16511655 .

31. Z. P. Huang, D. Z. Wang, J. G. Wen, M. Sennett, H. Gibson, and Z. F. Ren (2002), Applied Physics A: Materials Science \& Processing, 74: p. 387-391.

32. Z. P. Huang, J. W. Xu, Z. F. Ren, J. H. Wang, M. P. Siegal, and P. N. Provencio (1998), Applied Physics Letters, 73: p. 3845-3847.

33. S. lijima (1991), Nature, 354: p. 56-58.

34. S.-H. Jeong, H.-Y. Hwang, K.-H. Lee, and Y. Jeong (2001), Applied Physics Letters, 78: p. 2052-2054. 
35. N. Jiang, R. Koie, T. Inaoka, Y. Shintani, K. Nishimura, and A. Hiraki (2002), Applied Physics Letters, 81: p. 526-528.

36. V. Jourdain, O. Stephan, M. Castignolles, A. Loiseau, and P. Bernier (2004), Advanced Materials, 16: p. 447-453.

37. C. Journet, W. K. Maser, P. Bernier, A. Loiseau, M. L. d. 1. Chapelle, P. D. S. Lefrant, R. Lee, and J. E. Fischer (1997), Nature, 388; p. 756-758.

38. R. Kamalakaran, M. Terrones, T. Seeger, P. Kohler-Redich, M. Ruhle, Y. A. Kim, T. Hayashi, and Endo (2000), Applied Physics Letters, 77: p. 3385-3387.

39. H. Kind, J.-M. Bonard, C. Emmenegger, L.-O. Nilsson, K. Heradi, E. MailardSchaller, L. Schapbach, L. Forro, and K. Kern (1999), Advanced Materials, 11: p. 1285-1289.

40. C. Klinke, J.-M. Bonard, and K. Kern (2001), Surface Science, 492: p. 195-201.

41. N. Koratkar, B. Wei, and P. M. Ajayan (2002), Advanced Materials, 14: p. $997-$ 1000 .

42. W. Kraschmer, L. D. Lamb, K. Fostiropoulos, and D. R. Huffman (1990), Nature, 347: p. 354-358.

43. C. J. Lee and J. Park (2000), Applied Physics Letters, 77: p. 3397-3399,

44. C.-J. Lee and J.-E. Yoo (2000), Mass Synthesis Method of High Purity Carbon Nanotubes Vertically Aligned over Large-size Substrate using Thermal Chemical Vapour Deposition, Patent, EP 1059266 A2

45. J. Li, C. Papadopoulos, J. M. Xu, and M. Moskovits (1999), Applied Physics Letters, 75: p. 367-369.

46. W. Z. Li, J. G. Wen, and Z. F. Ren (2002), Applied Physics A: Materials Science \& Processing, 74: p. 397-402.

47. W. Z. Li, S. S. Xie, L. X. Qian, B. H. Chang, B. S. Zou, W. Y. Zhou, R. A. Zhao, and G. Wang (1996), Science, 274: p. 1701-1704.

48. Y.-L. Li, I. A. Kinloch, and A. H. Windle (2004), Science, 304: p. 276-278.

49. O. A. Louchev, Y. Sato, and H. Kanda (2002), Applied Physics Letters, 80: p. $2752-2754$.

50. A. H. Mahan, J. L. Alleman, M. J. Heben, P. A. Parilla, K. M. Jones, and A. C. Dillon (2002), Applied Physics Letters, 81: p. 4061-4063. 
51. V. I. Merkulov, D. H. Lowndes, Y. Y. Wei, G. Eres, and E. Voeelkl (2000), Applied Physics Letters, 76: p. 3555-3557.

52. T. Modl, N. Koratkar, E. Lass, B. Wei, and P. M. Ajayan (2003), Nature, 424: p. 171-174.

53. O. A. Nerushev, M. Sveningsson, L. K. L. Falk, and F. Rohmund (2001), Journal of Materials Chemistry, 11: p. 1122-1132.

54. J. Ouellete (2003), The industrial physicist: p. 18-21.

55. C. N. R. Rao, G. U. Kulkarni, A. Govindaraj, B. C. Satishkumar, and P. J. Thomas (2000), Pure Applied Chemistry, 72: p. 21-33.

56. C. N. R. Rao, R. Sen, B. C. Satishkumar, and A. Govindaraj (1998), Chemical Communication: p. 1525-1526.

57. Z. F. Ren, Z. P. Huang, J. W. Xu, J. H. Wang, P. Bush, M. P. Siegal, and P. N. Provencio (1998), Science, 282: p. 1105-1107.

58. A. A. Setlur, S. P. Doherty, J. Y. Dai, and R. P. H. Chang (2000), Applied Physics Letters, 76: p. 3008-3010.

59. J. I. Sohn, S. Lee, Y.-H. Song, S.-Y. Choi, K.-I. Cho, and K.-S. Nam (2001), Current Applied Physics, 1: p. 61-65.

60. M. Terrones, N. Grobert, J. Olivares, J. P. Zhang, H. Terrones, K. Kordatos, W. K. Hsu, J. P. Hare, P. D. Townsend, K. Prassides, A. K. Cheetham, H. W. Kroto, and D. R. M. Walton (1997), Nature, 388: p. 52-55.

61. A. Thess, R. Lee, P. Nikolaev, H. Dai, P. Petit, J. Robert, C. Xu, Y. H. Lee, S. G. Kim, A. G. Rinzler, D. T. Colbert, G. E. Scuseria, D. Tomanek, J. E. Fisher, and R. E. Smalley (1996), Science: p. 483-487.

62. Y. Tu, Z. P. Huang, D. Z. Wang, J. G. Wen, and Z. F. Ren (2002), Applied Physics Letters, 80: p. 4018-4020.

63. R. Vajtai, B. Wei, Y. J. Jung, A. Cao, S. K. Biswas, G. Ramanath, and P. M. Ajayan (2003), IEEE Transactions on Nanotechnology, 2: p. 355-361.

64. X. Wang, Y. Liu, P. a. Hu, G. Yu, K. Xiao, and D. Zhu (2002), Advanced Materials, 14: p. 1557-1559.

65. B. Wei, Z. J. Zhang, G. Ramanath, and P. M. Ajayan (2000), Applied Physics Letters, 77: p. 2985-2987. 
66. B. Q. Wei, R. Vajtai, Y. Jung, J. Ward, R. Zhang, G. Ramanath, and P. M. Ajayan (2002), Nature, 416: p. 495-496.

67. Y. Y. Wei, G. Eres, V. I. Merkulov, and D. H. Lowndes (2001), Applied Physics Letters, 78: p. 1394-1396.

68. W. Xu, T. Kyotani, B. K. Pradhan, T. Nakajima, and A. Tomita (2003), Advanced Materials, 15: p. 1087-1090.

69. Y. Yang, S. Huang, H. He, A. W. H. Mau, and L. Dai (1999), Journal of American Chemical Society, 121: p. 10832-10833.

70. E. Yenimez, Q. Wang, R. J. Chen, D. Wang, and H. Dai (2002), Applied Physics Letters, 80: p. 2225-2227.

71. W. Yi, T. Jeong, S. Yu, J. Heo, C. Lee, J. Lee, W. Kim, J.-B. Yoo, and J. Kim (2002), Advanced Materials, 14: p. 1464-1468.

72. G. Zhang, D. Mann, L. Zhang, A. Javey, Y. Li, E. Yenilmez, Q. Wang, J. P. McVittie, Y. Nishi, J. Gibbons, and H. Dai (2005), Proceedings of the National Academy of Sciences of United States of America, 102: p. 16141-16145.

73. W. D. Zhang, Y. Wen, W. C. Tjiu, G. Q. Xu, and L. M. Gan (2002), Applied Physics A: Materials Science \& Processing, 74: p. 419-422.

74. Y. Zhang, A. Chang, J. Cao, Q. Wang, W. Kim, Y. Li, N. Morris, E. Yenilmez, J. Ling, and H. J. Dai (2001), Applied Physics Letters, 79: p. 3155-3157.

75. Z. J. Zhang, B. Q. Wei, G. Ramanath, and P. M. Ajayan (2000), Applied Physics Letters, 77: p. 3764-3766.

76. H. W. Zhu, C. L. Xu, D. H. Wu, B. Q. Wei, R. Vajtai, and P. M. Ajayan (2002), Science, 296: p. 884. 


\section{Chapter 3 \\ Synthesis and Characterization of Multi-walled Carbon Nanotubes by Chemical Vapour Deposition}

This chapter describes my own experimental work on the synthesis and characterization of MWNTs by chemical vapour deposition method. Also the characterization results and the discussion of them will be presented here.

This part of work involves the synthesis of MWNTs by CVD from different precursors: $\mathrm{Fe}\left(\mathrm{NO}_{3}\right)_{3}$, ferrocene and $\mathrm{FePC}$. In the synthesis experiment, a dual-furnace system was adopted and acetylene was used as the carbon source, argon and hydrogen were used as carrier gas and reducing agent, respectively.

The characterization of the MWNT samples involved scanning electron microscopy, transmission electron microscopy, energy dispersive X-ray analysis, Raman spectroscopy and thermogravimetric analysis.

\subsection{Synthesis setup}

\section{Raw materials}

1. $\mathrm{Fe}\left(\mathrm{NO}_{3}\right)_{3} \cdot 9 \mathrm{H}_{2} \mathrm{O}, 99.5 \%$ pure, purchased from Aldrich.

2. Ferrocene, $\mathrm{Fe}\left(\mathrm{C}_{5} \mathrm{H}_{5}\right)_{2}, 98 \%$ pure, and iron(II) phthalocyanine, $\mathrm{FeC}_{32} \mathrm{~N}_{8} \mathrm{H}_{16}(\mathrm{FePC})$, 99.8\% pure, both purchased from Aldrich. 
Chapter 3 Synthesis and Characterization of Multi-walled Carbon Nanotubes by Chemical Vapour Deposition

3. Argon, Ar, $98 \%$ pure, hydrogen, $\mathrm{H}_{2}, 99 \%$ pure, and acetylene, $\mathrm{C}_{2} \mathrm{H}_{2}, 98 \%$ pure, all purchased from BOC Gas New Zealand.

\subsubsection{Synthesis of carbon nanotubes from $\mathrm{Fe}\left(\mathrm{NO}_{3}\right)_{3}$-derived nanoparticles}

The synthesis of carbon nanotubes from $\mathrm{Fe}\left(\mathrm{NO}_{3}\right)_{3}$-derived catalysts follows the route as described in the references $[3,4,15]$ and below:

A quartz glass tube, with $35 \mathrm{~mm}$ diameter and $1000 \mathrm{~mm}$ length, was placed inside a furnace equipped with a temperature controller. The growth substrate and the catalyst were prepared as follows: A piece of $\mathrm{SiO}_{2}$ silica glass $(1 \mathrm{~mm} \times 1 \mathrm{~mm})$, was cleaned with ethanol and acetone followed by drying with an airflow from an airgun. A solution of $\mathrm{Fe}\left(\mathrm{NO}_{3}\right)_{3} \cdot 9 \mathrm{H}_{2} \mathrm{O}\left(10 \mu \mathrm{L}\right.$ of $\left.0.1 \mathrm{~mol} \cdot \mathrm{dm}^{-3}\right)$ in ethanol was placed on the top of the silica glass as the growth substrate for deposition of MWNTs. The substrate was placed in a silica glass tube reactor heated by the furnace after it was stored in an oven at $80^{\circ} \mathrm{C}$ overnight to completely remove the ethanol from the substrate.

A single-furnace CVD was carried out in the following way: The inside of the tube reactor was purged in a flow of argon at a flowrate of $800 \mathrm{sccm}$ (cubic centimetre per minute) and then a stream of hydrogen was introduced into the reactor with a flowrate of $100 \mathrm{sccm}$ for 30 minutes at a temperature of $800{ }^{\circ} \mathrm{C}$ to reduce the $\mathrm{Fe}^{3+}$ from the precursor. Then the carbon source, $\mathrm{C}_{2} \mathrm{H}_{2}$ with a flowrate of $20 \mathrm{sccm}$ was flowed into the tube, carried by Ar at $500 \mathrm{sccm}$ and $\mathrm{H}_{2}$ at $100 \mathrm{sccm}$ while the temperature was raised and kept at $850{ }^{\circ} \mathrm{C}$. After a reaction for 10 minutes, the furnace was switched off and the reactor was cooled to room temperature in an argon atmosphere. Samples were scraped off from the surface of the substrate for characterizations. 
Chapter 3 Synthesis and Characterization of Multi-walled Carbon Nanotubes by Chemical Vapour Deposition

\subsubsection{Synthesis of aligned MWNTs by a dual furnace CVD}

\subsubsection{Experimetal setup}

The experimental set-up of a dual furnace CVD we employed follows the reports from different sources $[5,9-11,13,20,21,23-25]$ and can be described as follows: A piece of silica tube, with $35 \mathrm{~mm}$ diameter and $1000 \mathrm{~mm}$ length, was placed in a dual furnace system, which consists of a sublimation furnace, in which precursors are placed and a deposition furnace, in which MWNTs are grown (The diagram of the apparatus was depicted in Figure 3-1). Two temperature controllers, which equipped with thermocouples, controlled the furnace temperatures separately. A ceramic dish, which contained approximately $0.1 \mathrm{~g}$ precursors, ferrocene or FePC, was placed inside the silica tube in the sublimation furnace. A piece of cleaned (the method of cleaning is the same as mentioned above) quartz plate $(15 \mathrm{~mm} \times 10 \mathrm{~mm})$ was placed in the deposition furnace as the growth substrate for MWNTs.

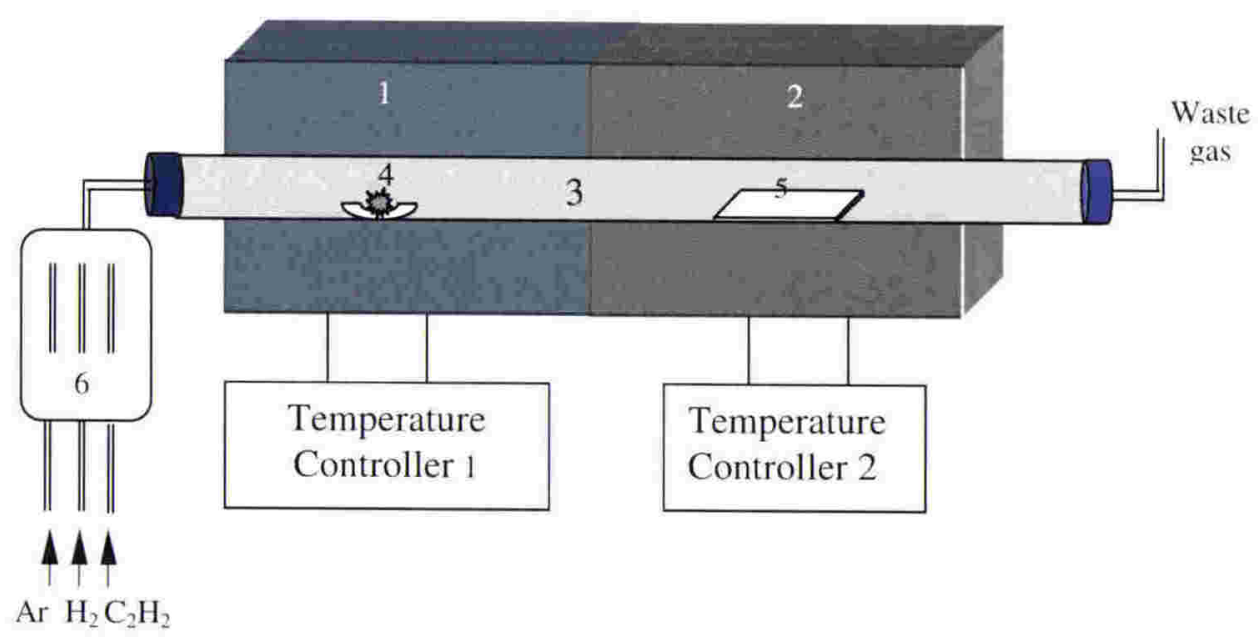

Figure 3-1 A diagram of a dual-furnace CVD system for synthesizing aligned MWNTs. 1. Sublimation furnace; 2. Deposition furnace; 3. Silica tube; 4. Ceramic dish; 5. Silica glass; 6. Flowrate meter. 
Chapter 3 Synthesis and Characterization of Multi-walled Carbon Nanotubes by Chemical Vapour Deposition

\subsubsection{Procedure}

A dual-furnace CVD process takes place as follows: Once the deposition furnace reached the desired temperature under the atmosphere of argon at a flowrate of $500 \mathrm{sccm}$ and hydrogen at a flowrate of $100 \mathrm{sccm}$, the temperature of the sublimation furnace was raised $60{ }^{\circ} \mathrm{C}$ per minute to $450{ }^{\circ} \mathrm{C}$ and $650^{\circ} \mathrm{C}$ in the case of ferrocene and $\mathrm{FePC}$, respectively. Then a stream of acetylene at a flowrate of $15-25 \mathrm{sccm}$ was introduced. The reaction was allowed to proceed for $10-20$ minutes at a temperature from $800{ }^{\circ} \mathrm{C}$ to 900 ${ }^{\circ} \mathrm{C}$. Then the flow of acetylene was stopped and the furnace was switched off, with argon and hydrogen gases purging the inside of the silica tube until the tube reactor was completely cooled.

\subsubsection{Pre-templated synthesis of aligned MWNTs}

Under the dual-furnace CVD setup as described in the previous section, the silica glass in the deposition furnace was replaced by a temperated micron-sized $\mathrm{Si}_{3} \mathrm{~N}_{4}$ substrate, on which there were gold wires, aluminium patterns and a company logos "INDUSTRIAL RESEARCH LIMITED", which had been printed positively in $\mathrm{Si}_{3} \mathrm{~N}_{4}$ and lifted $1 \mu \mathrm{m}$ above the substrate. Each letter of the company logo is $10 \mu \mathrm{m}$ long and $10 \mu \mathrm{m}$ wide. SEM observation was made before and after deposition. The growth of CNTs on the microstructure was made at $850^{\circ} \mathrm{C}$ by dual-furnace CVD.

\subsection{Characterization}

\subsubsection{Scanning Electron Microscopy (SEM)}

1. Instruments

(1) Low-resolution SEM (LRSEM) 
Chapter 3 Synthesis and Characterization of Multi-walled Carbon Nanotubes by Chemical Vapour Deposition

LRSEM microscopy of CNTs was collected on a Philips EM 505 Scanning Electron Microscope.

(2) High-resolution SEM (HRSEM)

HRSEM of CNTs was made on a Jeol 6500F Field Emission Scanning Electron Microscope.

(3) Energy Dispersive X-ray (EDX) spectra

EDX spectra of CNTs were performed on an EX-23000BU energy dispersive X-ray detector (EDX), which was coupled to a Jeol 6500F Field Emission Scanning Electron Microscope.

2. Sample preparations and SEM setup

The samples under investigation include the MWNTs with catalysts derived from $\mathrm{Fe}\left(\mathrm{NO}_{3}\right)_{3}$, ferrocene and $\mathrm{FePC}$.

A sample of carbon nanotubes was peeled off the substrate with a razor blade and stuck to a piece of carbon tape mounted on a SEM sample holder, followed by sputter-coating the sample with a layer of 4-6 nm Au or Pt. Samples derived from ferrocene and FePC were retained in films.

The samples for EDX were prepared in the same way as above except without being sputter coated.

The operating voltage of the SEM was in the variety of 5.0, 8.0, 10.0, 12.0 and $15.0 \mathrm{kV}$ for both high- and low- resolution SEM.

\subsubsection{Transmission Electron Microscopy (TEM)}

1. Instrument 
Chapter 3 Synthesis and Characterization of Multi-walled Carbon Nanotubes by Chemical Vapour Deposition

Transmission electron microscopy of CNTs was measured on a Philips EM 400 transmission electron microscope.

2. Sample preparations and TEM setups

Carbon nanotubes were peeled off from the growth substrate and a trace amount of the sample was suspended in $95 \%$ ethanol, assisted by ultrasonication water bath for 10 minutes. A drop of the carbon nanotubes suspension was transfered onto a carbon-coated copper TEM grid by a glass pipette and was allowed to dry in air. Then the grid was mounted onto a TEM sample holder.

The samples being investigated included MWNTs made with catalysts derived from $\mathrm{Fe}\left(\mathrm{NO}_{3}\right)_{3}$, ferrocene and $\mathrm{FePC}$.

3. The operating voltage of TEM was $120 \mathrm{kV}$.

\subsubsection{High Resolution Transmission Electron Microscopy}

1. Instrument

High-resolution Transmission Electron Microscopy (HRTEM) of CNTs was performed on a Jeol 2011 Transmission Electron Microscope.

2. Sample

The CNT samples under HRTEM investigation included MWNTs prepared with catalysts derived from $\mathrm{Fe}\left(\mathrm{NO}_{3}\right)_{3}$, ferrocene and $\mathrm{FePC}$.

The sample preparation of HRTEM was the same as that of TEM described in the TEM experimental section.

3. The operating voltage of HRTEM was $200 \mathrm{kV}$, the current was kept at $105 \mu \mathrm{A}$. 
Chapter 3 Synthesis and Characterization of Multi-walled Carbon Nanotubes by Chemical Vapour Deposition

\subsubsection{Raman spectroscopy}

1. Instrument

Jobin-Yvon LabRaman HR micro-Raman spectrometer, with a charge coupled device detector (CCD).

2. Instrument setup

The samples were placed on a piece of Si wafer. A laser with an excitation wavelength of $632.88 \mathrm{~nm}$ was used as the light source. The laser passed through a $100 \times$ objective lens, giving an illuminated spot of $4 \mu \mathrm{m}^{2}$. The incident light was parallel to the direction of the alignment of the CNTs. A typical acquisition time for a spectrum was 60 second.

3. Sampling

(1) MWNTs

Samples of CNTs were scraped off the growth substrate, retained in films and transferred to a piece of clean surface-oxidized Si wafer by tweezers. The samples include CNTs synthesized from ferrocene- and FePC-derived catalysts. Raman studies were carried out on both the tip side and the root side of the aligned films of CNTs.

(2) SWNTs

Raman spectra of purified SWNT samples, which were purchased from c@Rice, were investigated. The sample was placed on a piece of clean surface-oxidized Si wafer.

\subsubsection{Thermogravimetric Loss Analysis (TGA)}

\section{Instrument}

Polymer Laboratory PL-STA (Simultaneous Thermal Analyzer) 1000H, was coupled with Rheometric Scientific Plus V5.41 analyzing software. 
Chapter 3 Synthesis and Characterization of Multi-walled Carbon Nanotubes by Chemical Vapour Deposition

2.Instrument setup and sampling

In a typical TGA measurement, the temperature was ramped at $20{ }^{\circ} \mathrm{C}$ per minute from 20 ${ }^{\circ} \mathrm{C}$ to $900{ }^{\circ} \mathrm{C}$ in air with a flowrate of $20 \mathrm{~mL}$ per minute. Weight loss and heat flow versus temperature were recorded and plotted. A sample of approximately $2-5 \mathrm{mg}$ of CNTs was held in a ceramic pan. The plot was corrected using the buoyancy method.

\subsection{Results and discussions}

\subsubsection{SEM and TEM investigations on carbon nanotubes from $\mathrm{Fe}\left(\mathrm{NO}_{3}\right)_{3}$-derived catalysts}

As shown in the SEM image of Figure 3-2, carbon nanotubes were produced from $\mathrm{Fe}\left(\mathrm{NO}_{3}\right)_{3}$-derived catalysts. Long tubular structures were observed from TEM images, as seen in Figure 3-3 (a) and (b). The greater-than-20 nm diameters and the thick sidewalls are clear evidences of MWNTs. The MWNTs are strongly curled and heavily entangled. Most of the black dots inside tubes are considered as catalyst particles, which have the same dimensions as the internal diameters of the CNTs. The diameters of the MWNTs range from 20 to $50 \mathrm{~nm}$. However, their exact lengths could not be determined from TEM and SEM images because of their highly entangled geometry.

As observed from the TEM images in Figure 3-3 (a) and (b), there exist large amount of impurities, and straight tubes over long distance were seldom spotted. The universal existence of curly structures suggests that there are a large number of defects fromed along the CNT walls during the growth.

As shown in Figure 3-3 (a), the catalyst particles are encapsulated in the tips. It can also be observed that catalyst particles are found in the knots of CNTs, in other words, some 
Chapter 3 Synthesis and Characterization of Multi-walled Carbon Nanotubes by Chemical Vapour Deposition

of the CNTs might share catalyst particles in their middle sections. These phenomena indicate that the tip-growth mechanism and the base-growth mechanism may occur simultaneously or one after another during the growth process. The growth process can be proposed as follows: the catalyst particles were lifted from the substrate during the growth of the tubes and some of catalysts still remained active as nucleation sites for coming carbon species to grow into tubes, while the other catalyst dots lost their catalytic abilities and closed the open tips of carbon tubules into domes.

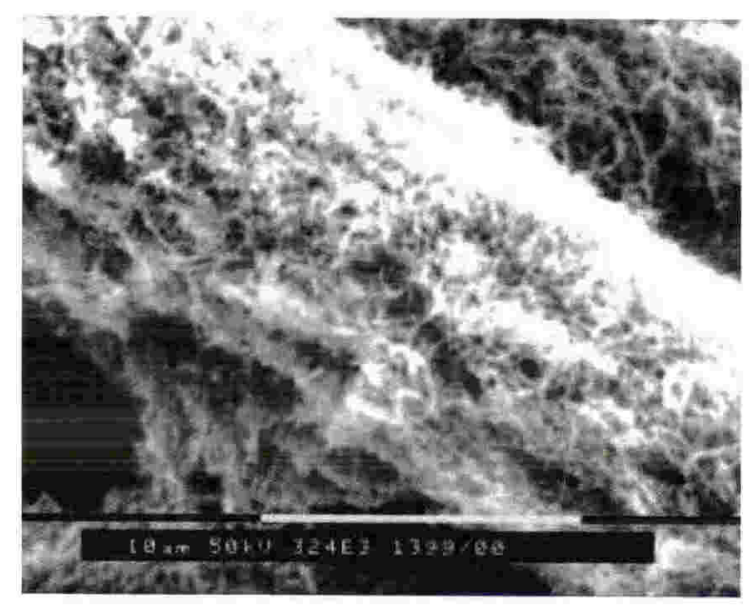

Figure 3-2 An SEM image of multi-walled carbon nanotubes using $\mathrm{Fe}^{3+}$-derived catalysts.
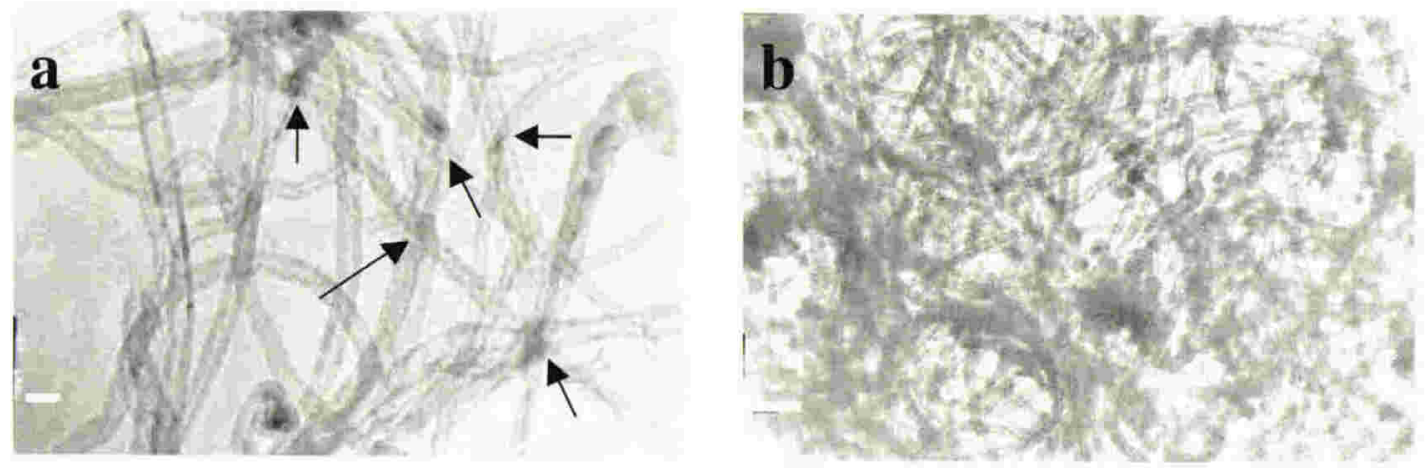

Figure 3-3 carbon nanotubes produced from $\mathrm{Fe}^{3+}$-derived catalysts. (a) In a moderate magnification, the scale bar denotes $50 \mathrm{~nm}$, the arrows in the figure indicate the dots which were shared by CNTs; (b) In a low magnification, the scale bar denotes $100 \mathrm{~nm}$. 
Chapter 3 Synthesis and Characterization of Multi-walled Carbon Nanotubes by Chemical Vapour Deposition

\subsubsection{SEM and TEM investigations on aligned carbon nanotubes}

\subsubsection{Synthesis of aligned MWNTs from ferrocene}

\section{SEM microscopy}

a. CNTs samples collected from the top surface of quartz plate

The low resolution SEM images, viewed from different angles of the samples, are demonstrated in Figure 3-4. As seen from these images, aligned carbon nanotubes were produced. The CNT bundles are perpendicular to the growth substrate and aligned in parallel with each other.
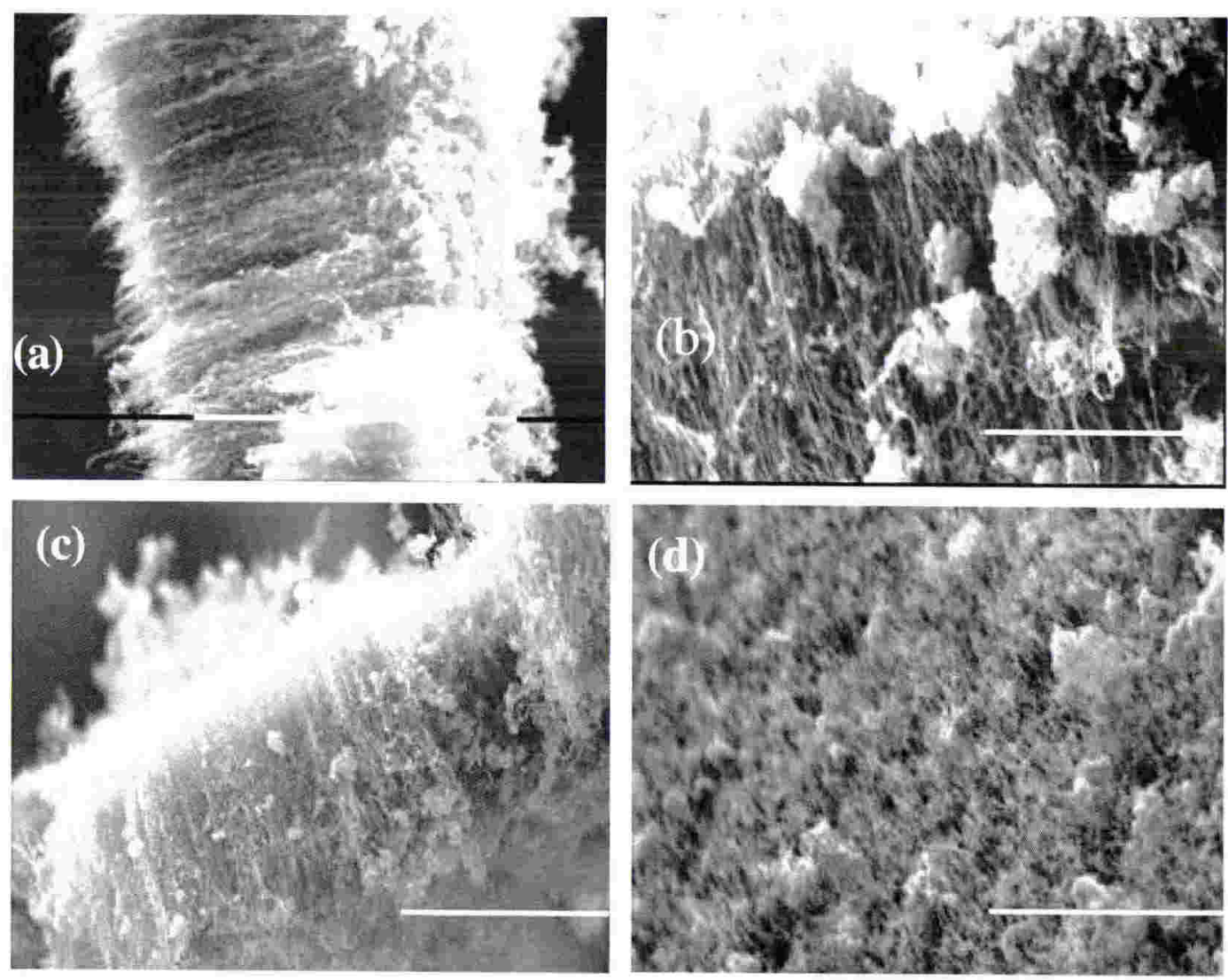

Figure 3-4 Low resolution SEM images of aligned carbon nanotube films using ferrocene as precursor: as-made CNTs which were peeled from the top surface of quartz plate, all the scale bars denote $10 \mu \mathrm{m}$ except in (b) which represents $5 \mu \mathrm{m}$. (a) The alignment is from the roots (on the right side of the photo) to the tops, (b) side view, (c) roots are toward the bottom side of the image, and (d) a view over the tips of CNTs. 
Chapter 3 Synthesis and Characterization of Multi-walled Carbon Nanotubes by Chemical Vapour Deposition

As shown in these SEM images, the average length of CNTs in the films was about 12 $\mu \mathrm{m}$. The actual lengths of the CNTs would probably be above this because of the large density of curves and bends found within their structures. All of the samples could be easily peeled off from the quartz glass and the alignment of the CNTs was kept intact. As shown in Figure 3-4 (b) and (c), there are some impurities attached to the outside walls of the aligned CNTs and also from Figure 3-4 (d), there are some irregular materials on the tips of the CNTs.

SEM investigation gives us a clear picture of CNT films and their alignment. However, the diameters of individual CNTs are enlarged in SEM images due to the charging effect on the surface of CNTs in the SEM chamber. A more precise characterization of the diameters of the individual CNTs is given by TEM microscopy, as presented in the next section.

\section{b. CNTs collected from the bottom surface of quartz plate}

The low-resolution SEM images of the CNTs collected from the bottom surface of the quartz plate are shown in Figure 3-5. These individual tubes are in straight forms. The lengths of individual CNTs are uniform at around $11 \mu \mathrm{m}$. Compared with the densely deposited top surface of the quartz plate, fewer CNTs grew on the bottom side of the substrate and were unable to hold the alignment into a layer. The reason for this difference can be proposed as follows: catalysts from sublimed precursors are much less likely to be available on the bottom surface than those on the upper side of the substrate. On the other hand, the vertical alignment described in the previous section, should have resulted from the large density of catalysts deposited before the growth of CNTs. 


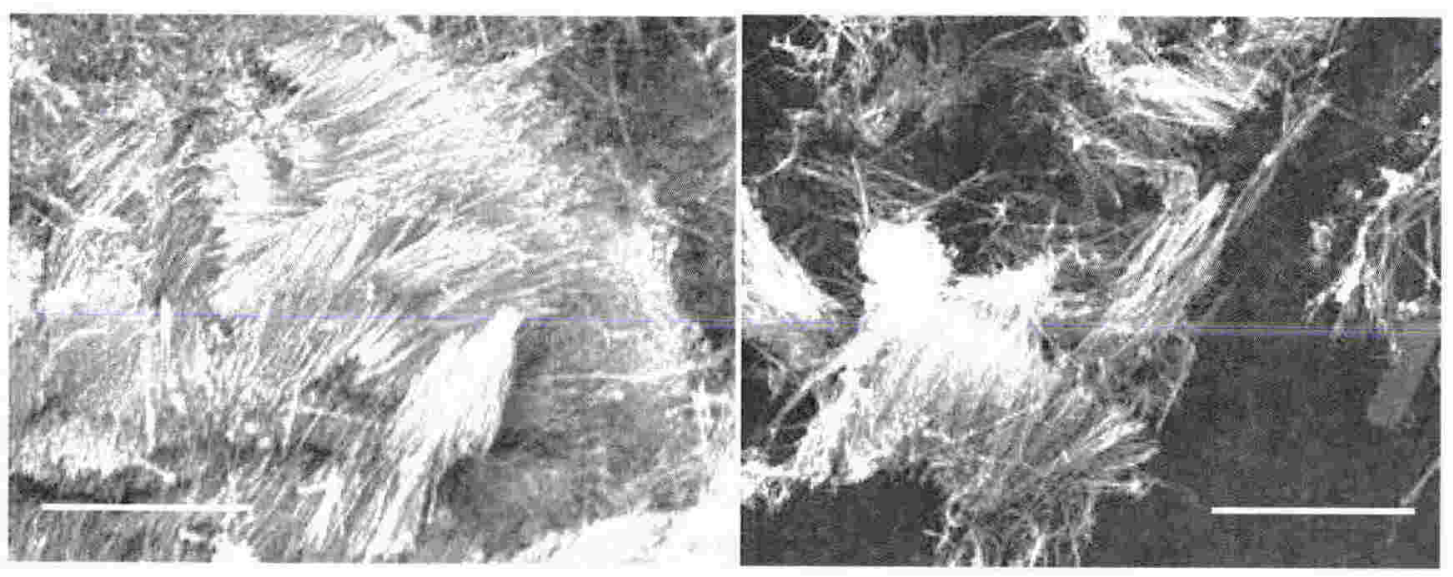

Figure 3-5 SEM images of CNTs collected from the bottom surface of the quartz plate. The scale bars above are $10 \mu \mathrm{m}$.

\section{c. Depositing carbon nanotubes on a pre-patterned $\mathrm{Si}_{3} \mathrm{~N}_{4}$ substrate}

A micro-structure with a layout as described elsewhere [14], is shown in Figure 3-6 (a).

The structure is based on a $\mathrm{Si}_{3} \mathrm{~N}_{4}$ substrate, with lift-up characters, "INDUSTRIAL RESEARCH LIMITED", which had been raised $1 \mu \mathrm{m}$ in $\mathrm{Si}_{3} \mathrm{~N}_{4}$ above the substrate, with each stroke $2 \mu \mathrm{m}$ wide, positively printed by a photo-lithographic technique. Besides, there are $\mathrm{Au}$ wires in thin strips and $\mathrm{Al}$ squares and wide strips which were fabricated on the $\mathrm{Si}_{3} \mathrm{~N}_{4}$ substrate, shown in white patterns and were indicated by a dotted arrow and solid arrows, respectively, in the SEM image shown in Figure 3-6 (a). The substrate was placed face up, in the deposition furnace as the growing substrate for vertically aligned carbon nanotubes from ferrocene precursors.

After CNTs were grown on, the microstructure was viewed under SEM, as shown in Figure 3-6 (b) and (d). The pattern of CNTs was found to have been faithfully following the pattern pre-printed in $\mathrm{Si}_{3} \mathrm{~N}_{4}$, from these images. After CNT growing, the pattern of "INDUSTRIAL RESEARCH LIMITED" was easily identified and well preserved. 
Chapter 3 Synthesis and Characterization of Multi-walled Carbon Nanotubes by Chemical Vapour Deposition

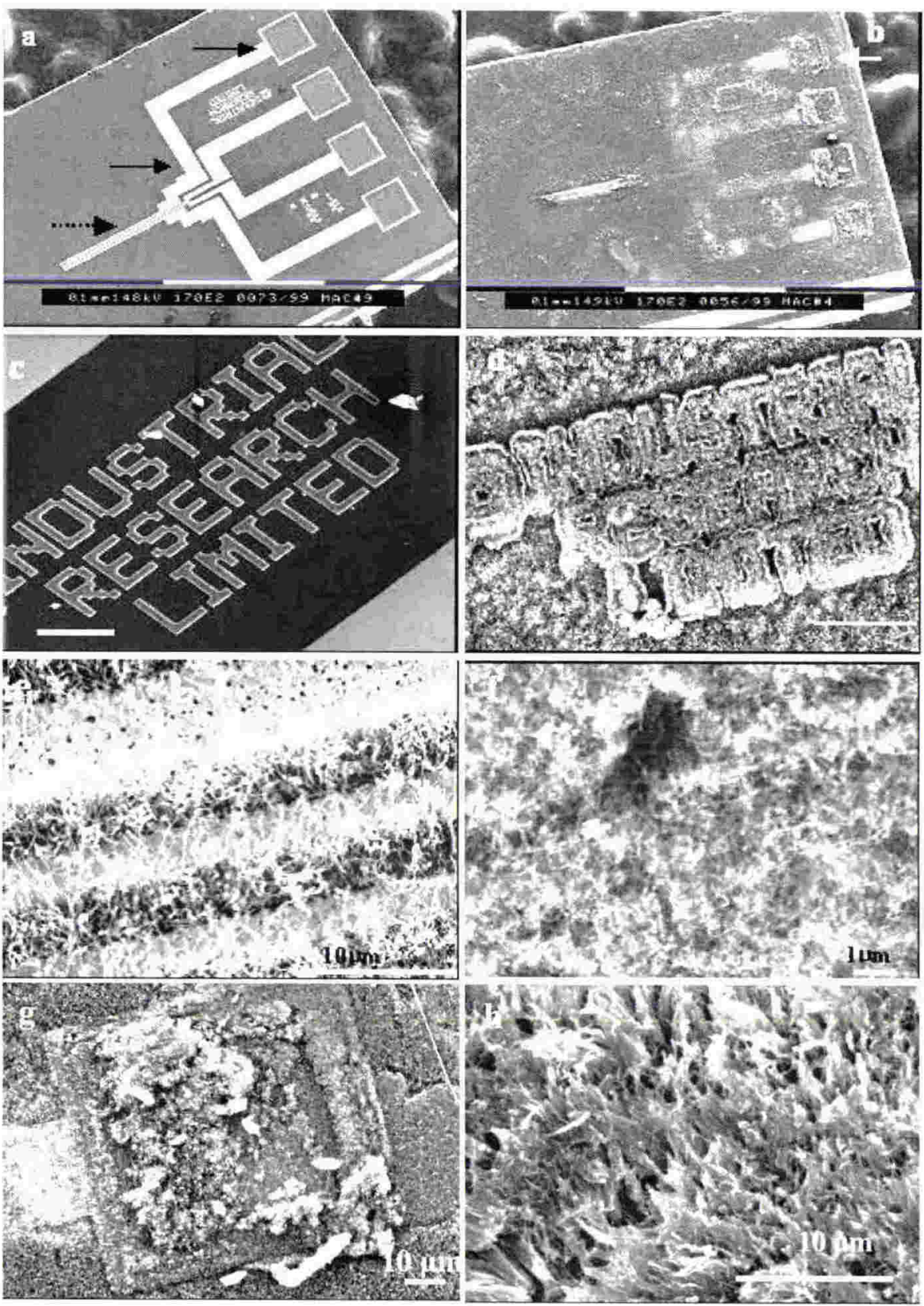

Figure 3-6 SEM images of aligned carbon nanotubes on a pre-patterned $\mathrm{Si}_{3} \mathrm{~N}_{4}$ substrate. (a) The original structure before the CNTs deposition. The dotted arrow and the solid arrows point to $\mathrm{Au}$ wires and $\mathrm{Al}$ wires, respectively. (b) The structure after CNT deposition. (c) A company logo before CNTs deposited on it. (d) The company logo after CNTs deposited. The scale bars in (c) and (d) are both $10 \mu \mathrm{m}$ each. (e) CNTs grown within the gaps between the Au wiresstrips (white region), which are $5 \mu \mathrm{m}$ apart. (f) An enlarged view over one of the letters in the company logo. (g) The substrate which was exposed after a $\mathrm{Al}$ pad (enlarged view from one of the four on the right of (b) melted, as pointed by the arrow in (b)). (h) A more detailed top view over CNTs as in (g). 
Chapter 3 Synthesis and Characterization of Multi-walled Carbon Nanotubes by Chemical Vapour

Deposition

However, compared with the print before CNTs' deposition, shown in Figure 3-6 (c), the CNT-deposited logo (see Figure 3-6 (d)) covers a slightly larger area than the temperlate itself. It suggests that the CNTs which grew on the sidewalls of the $\mathrm{Si}_{3} \mathrm{~N}_{4}$ templates, reached into the vincint spaces. Figure 3-6 (f) demonstrated a magnified view over one of the CNT-deposited letters in the logo.

In addition, it can be seen from Figure 3-6 (e) that aligned CNTs forests deposited on the $\mathrm{Si}_{3} \mathrm{~N}_{4}$ substrate while none of CNTs was found growing on the gold surfaces, which survived at $850{ }^{\circ} \mathrm{C}$ in the reaction chamber due to the high melting point of $\mathrm{Au}\left(1064^{\circ} \mathrm{C}\right)$. And it should also be mentioned here that there are some linkages built by the CNTs across the Au strips which are shown in white in the SEM image in Figure 3-6 (e). This can be extended to fabricating CNT-Au devices. On the other hand, the Al pad in figure 3-6 $(\mathrm{g})$ had been destroyed under the high temperature in the reaction chamber because of the low melting temperature of $\mathrm{Al}\left(660^{\circ} \mathrm{C}\right)$ and some of the melt $\mathrm{Al}$ was deposited on CNTs, as seen in the magnified view of Figure 3-6 (h).

\section{TEM microscopy}

As seen from the TEM images in Figure 3-7 (a) and (b), MWNTs were produced from ferrocene-derived catalysts. The diameters of CNTs from ferrocene-derived catalysts range from 20 to $60 \mathrm{~nm}$ and their major distributions are approximately from 40 to $50 \mathrm{~nm}$. Slightly entangled tubes are visible and they have smooth surfaces on their outer walls, However, there are some irregularities, which can be observed in the TEM images. The irregularities of the CNTs include the unevenly distributed thickness of their sidewalls and the non-uniform structures of their tubular shapes. There are some spherical catalyst 
particles, with diameters similar to those of the CNTs, encapsulated inside the CNTs.

These are suggested to be typical characteristics of the tip growth mechanism.
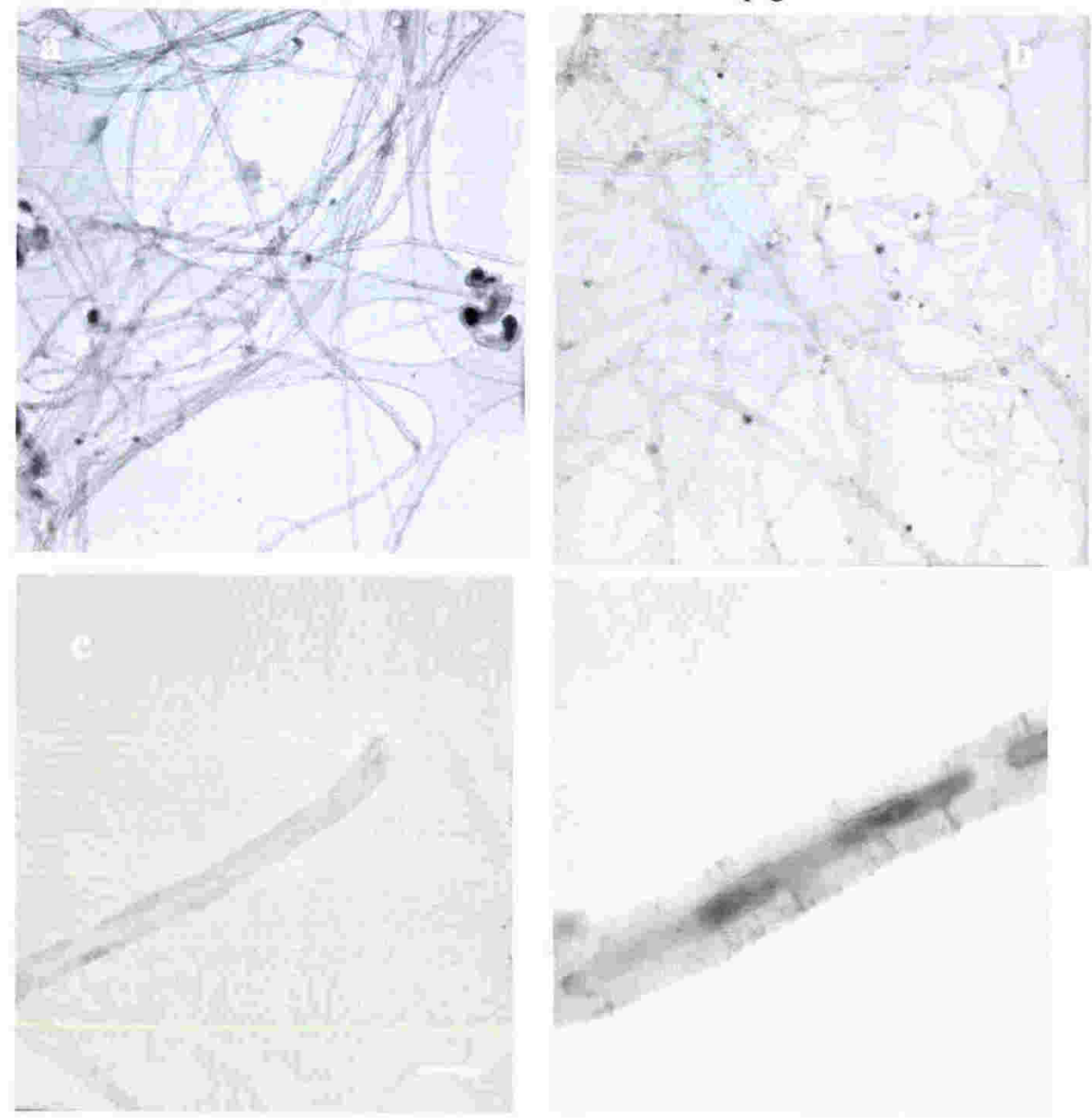

Figure 3-7 TEM images of carbon nanotubes produced from ferrocene-derived catalysts. (a), (b) TEM microscopy of CNTs with diameters ranging from $30-60 \mathrm{~nm}$ at low magnifications, scale bars denote $100 \mathrm{~nm}$, (c) a broken tip of CNT with about $40 \mathrm{~nm}$ diameter, and (d) a middle-section of carbon nanotube with a diameter of about $50 \mathrm{~nm}$, with catalyst particles, shown as black rod-like structures in it. Scale bars in (c) and (d) denote $50 \mathrm{~nm}$.

Images (c) and (d) in Figure 3-7 demonstrate a truncated tip and a middle section of two CNTs, with diameters of approximately 30 and $50 \mathrm{~nm}$, respectively. These TEM images gave further details of individual CNTs and demonstrated that the CNTs from this precursor contained a generally straight but individually irregular geometry. The open 
Chapter 3 Synthesis and Characterization of Multi-walled Carbon Nanotubes by Chemical Vapour Deposition

end in Figure 3-7 (c) might be caused by the ultrasonic treatment during the sample preparation before TEM microscopy. In Figure 3-7 (d), compartments containing catalyst-derived impurities are enclosed in a CNT structure. These impurities might have been lifted up from the pre-deposited catalyst particles on the substrate during a base growth process. All of these observations suggest that both the tip- and base-growth mechanisms might exist during their growth.

In addition, it should be mentioned that the alignment of ferrocene-derived MWNTs was lost after the ultrasonic treatment for TEM microscopy. The binding between CNT bundles results from van der Waals forces between CNTs. This suggests that a mild mechanical treatment on CNTs disintegrates the alignment between these CNT bundles. Thus there are no strong bindings between these CNTs.

\subsubsection{Synthesis of aligned MWNTs from iron (II) phthalocyanine (FePC)}

\section{SEM microscopy}

The LRSEM images in Figure 3-8 were given on aligned carbon nanotube films, which were formed on the top surface of a quartz plate using $\mathrm{FePC}$ as the precursors at $850{ }^{\circ} \mathrm{C}$ in a growth time of 15 minutes. All of the CNTs are of approximately the same height of $10 \mu \mathrm{m}$. A large area of approximately $2 \mathrm{~mm} \times 2 \mathrm{~mm}$ of aligned CNTs film was shown as Figure 3-8 (a). Apart from those similarities with the samples produced from ferrocene, the samples formed from FePC contain fewer impurities and a larger area of alignment, as seen from Figure 3-8 (a) and (b). Also, as shown from the side views of CNTs film in Figure 3-8 (d), these CNTs are straighter than the ferrocene-derived samples. 
Chapter 3 Synthesis and Characterization of Multi-walled Carbon Nanotubes by Chemical Vapour Deposition
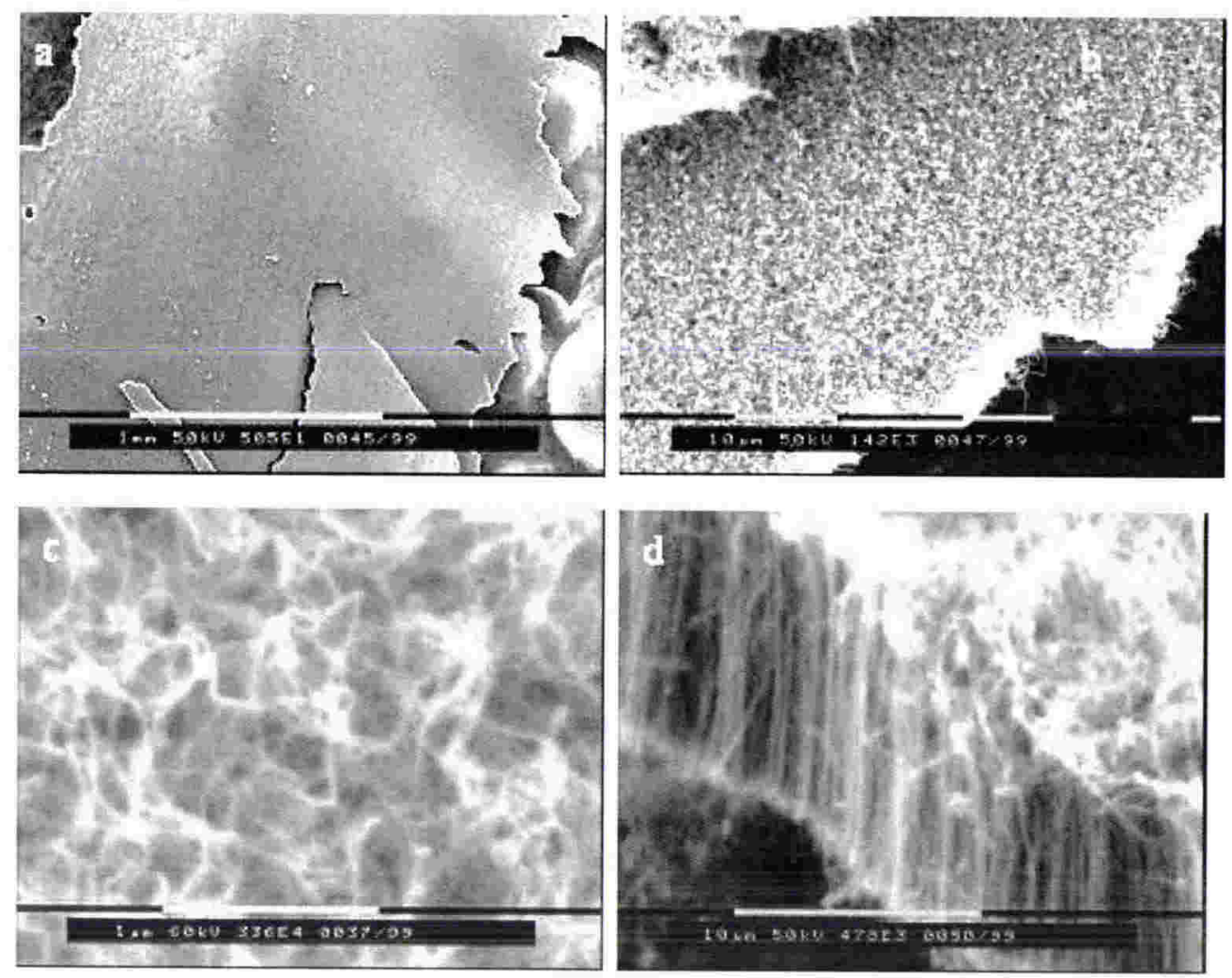
of the catalyst particles stay inside the bases of the CNTs and most of the tips are free of encapsulated particles.

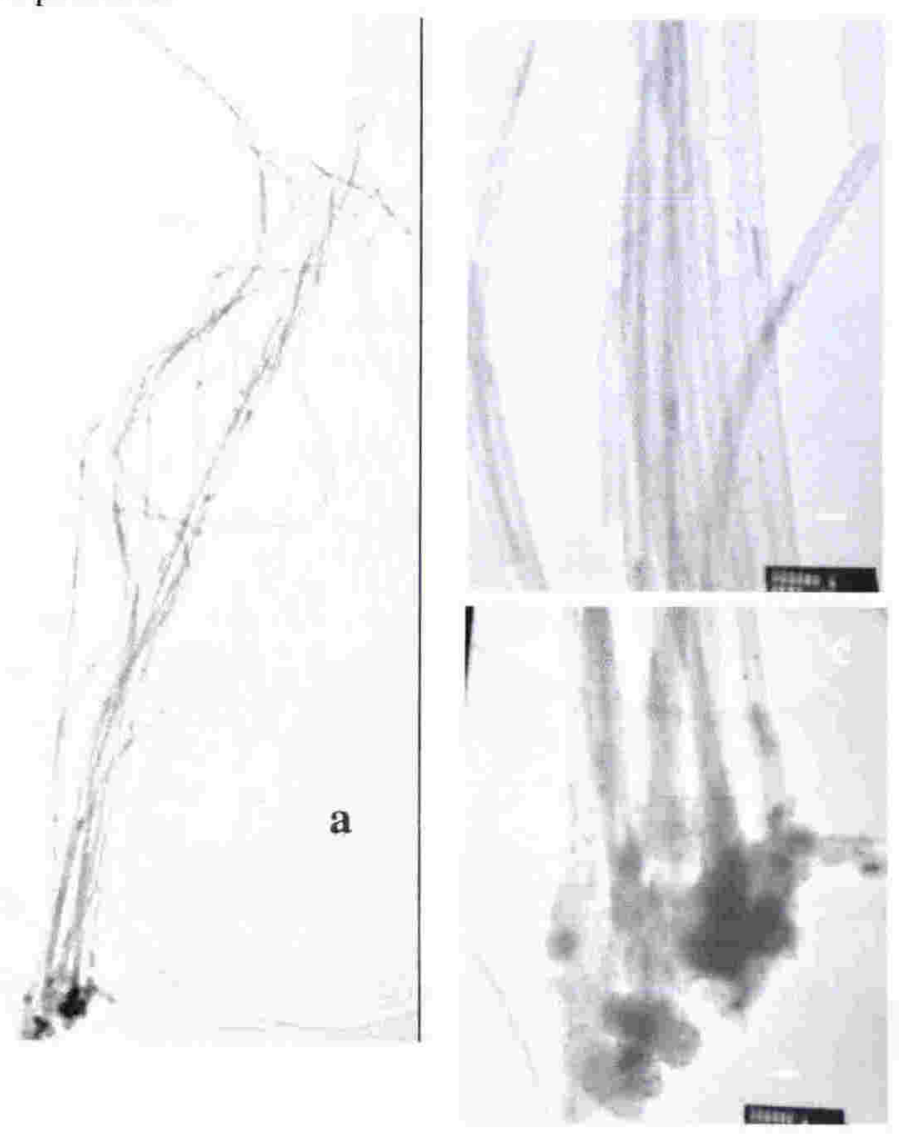

Figure 3-9 TEM images on a bundle of CNTs from FePC- derived catalysts, with roots attached together. The view (a) of a CNT bundle, from head to toe. The scale bar denotes $100 \mathrm{~nm}$, (b) magnified at the middle section of the bundle, the scale bar denotes $50 \mathrm{~nm}$, and (c) magnified at the root of the bundle, and the scale bar denotes $50 \mathrm{~nm}$.

Figure 3-9 (c) gave a magnified image of the roots of the MWNT bundles. Metal or metal-carbon alloy islands, which are in irregular shapes, with dimensions ranging from tens of nanometers to several hundreds of nanometers, were completely covered by carbon structures. The shapes and the dimensions of these catalyst islands did not confine the diameters of the CNTs extruding from them. This phenomenon has been supposed to be one of the main characteristics of the base-growth model [17]. The structures around 
the base ends of CNTs are much more irregular than those in the vincity of their middle sections and their tips. Some of the catalyst particles were carried upward from the main body of the catalysts in the base by the growing tubules and were trapped in the lower portions of the tubes during the growth process.
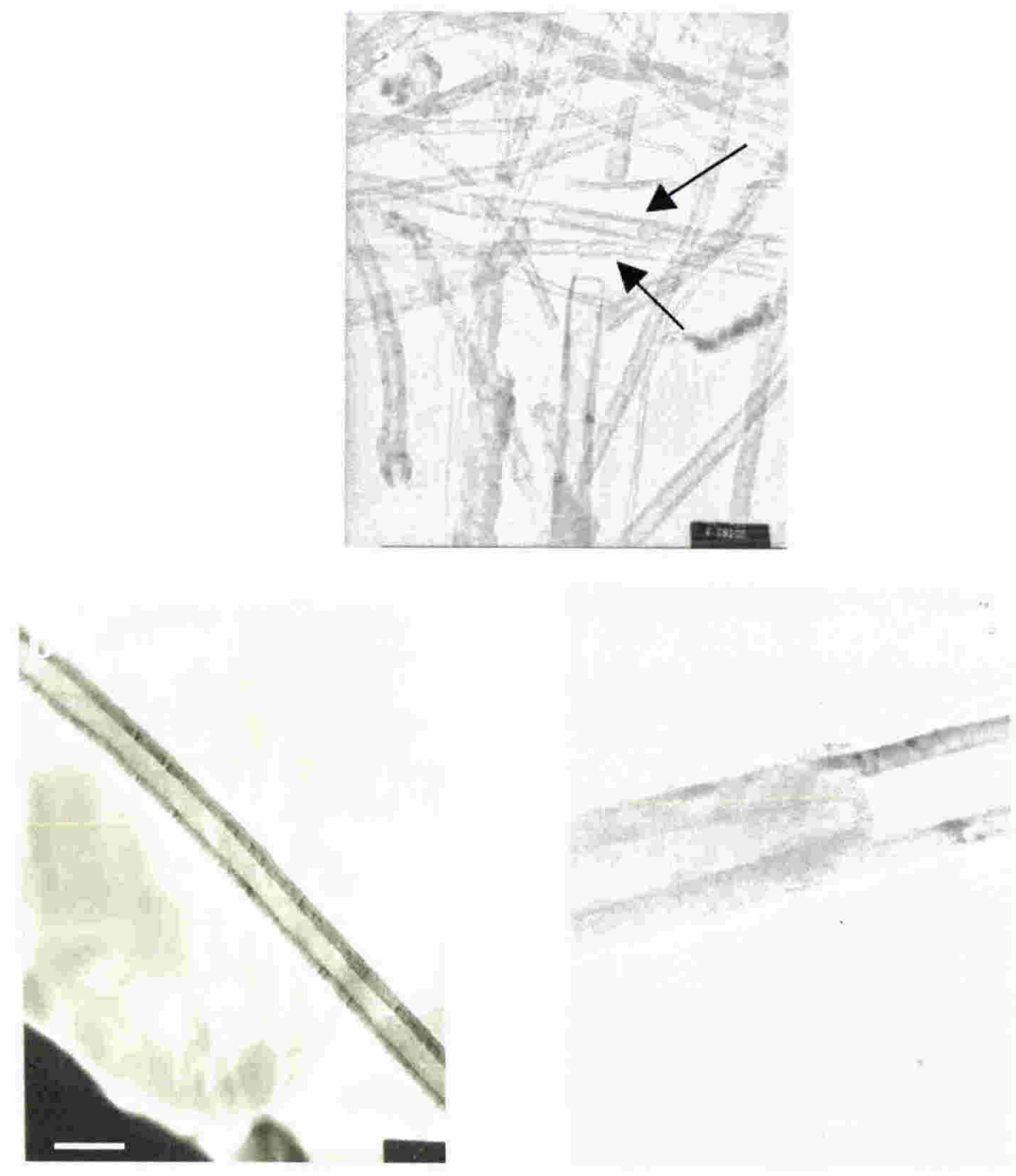

Figure 3-10 Middle sections of CNTs from FePC-derived catalysts. (a) CNTs in different forms: straight sections, bamboo structures, helix-shaped and broken tips. The arrows point to CNTs with bamboo strucutres. (b) Middle section of an individual tube. (c) A magnified view of bamboo structure inside a tube. The scale bars denote: a. $50 \mathrm{~nm}$, b. 50 $\mathrm{nm}, \mathrm{c.} 100 \mathrm{~nm}$. 
Chapter 3 Synthesis and Characterization of Multi-walled Carbon Nanotubes by Chemical Vapour Deposition

TEM images on the middle sections of the FePC-derived CNTs are shown in Figure 3-10. A large portion of the middle sections of the CNTs are free of impurities and well graphitized. Compared with CNTs from ferrocene-derived catalysts, these tubes are straighter and less entangled. However, some CNTs still contain impurities, which were either trapped inside their walls, or confined in their roots.

Bamboo structures, which are the capped graphitic structures trapped inside a CNT, are observed in the TEM images in Figure 3-10 (a) and (c). It can be seen, particularly from Figure 3-10 (a) that the curvature of these structures is pointing to the growing direction. The bamboo structure can be attributed to the periodic closure of CNT walls into cones and the growing of graphitic layers beneath them [17]. The bamboo structures without encapsulated particles and tips without particles are two pieces of strong evidences that CNTs from FePC-derived catalysts have been grown via the base-growth mechanism [17]. The distribution of catalysts inside CNT samples is to be given by Energy Dispersive X-Ray (EDX) analysis, which will be discussed in section 3.3.4.

\subsubsection{Investigation on nanostructures inside individual MWNTs by High Resolution TEM}

HRTEM images provide us with much clearer view over the morphology of individual CNTs than the LRTEM micrscopies we obtained in the earlier sections. The HRTEM in Figure 3-11 displayed a magnified and better-resoluted view over a section of MWNTs from $\mathrm{Fe}\left(\mathrm{NO}_{3}\right)_{3}$ which contained plenty of irregular carbon nanostructures. The MWNTs from $\mathrm{Fe}\left(\mathrm{NO}_{3}\right)_{3}$ precursors contain plenty of defects and curves, some of which were indicated by the arrows in the figure. The interlattice spacings in these MWNTs are all $0.34 \mathrm{~nm}$, which is one of the typical characteristics of a MWNT. 
Chapter 3 Synthesis and Characterization of Multi-walled Carbon Nanotubes by Chemical Vapour Deposition

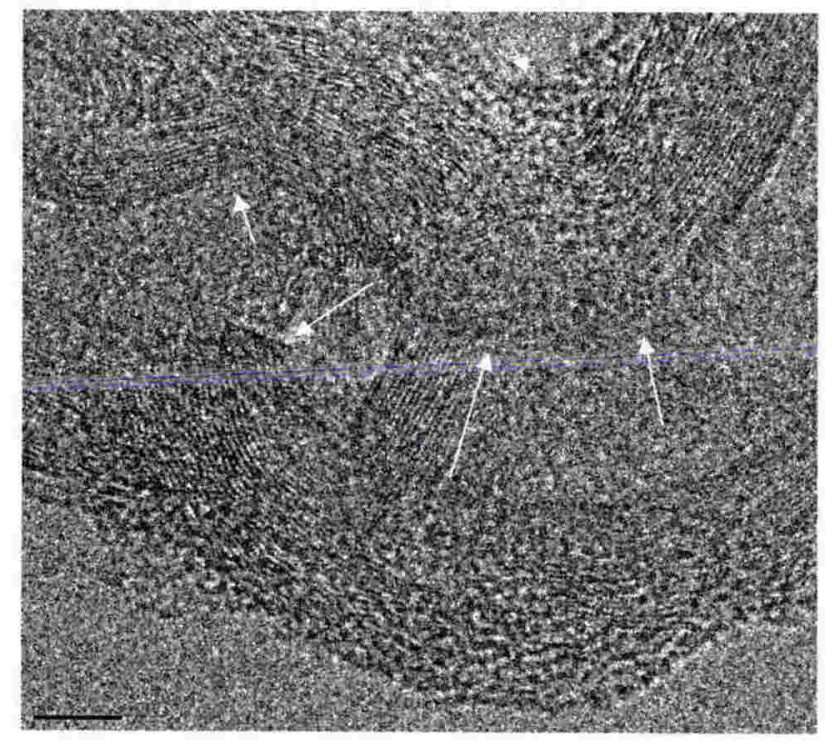

Figure 3-11 A HRTEM image of stacked MWNTs from $\mathrm{Fe}\left(\mathrm{NO}_{3}\right)_{3}$ precursor. As indicated by the white arrows, there are plenty of defects in the morphology of this genre of MWNTs. However, interlattice spacings of $0.34 \mathrm{~nm}$ can be identified in the tube sections in this image. The scale bar represents $5 \mathrm{~nm}$.

Inside individual MWNTs, which were made from ferrocene and FePC precursors, as shown in Figure 3-12, crystalline structures in fringes were found. As reviewed in Chapter 1, these fringes were projections of shells in concentric nanostructures. The MWNTs possess co-axial nanotube structures, in another word, the nanotubular layers inside MWNTs are concentric with one axis, as shown in Figure 3-12 (a), a section of an individual MWNT from ferrocene-derived catalyst.

A structure with concentric graphitic shells is supposed to be the key characteristic of MWNTs and it has been observed in both MWNTs from ferrocene and FePC precursors, as demonstrated in Figure 3-12 (a) and (b). The CNT section demonstrated in Figure 3-12 (a) has an outer diameter of $25 \mathrm{~nm}$ and an inner diameter of $11 \mathrm{~nm}$. It has more than twenty shells in its structures. Also it should be mentioned here that in the left top corner of this image is a capped catalyst particle, encapsulated by a bamboo compartment. 
Chapter 3 Synthesis and Characterization of Multi-walled Carbon Nanotubes by Chemical Vapour Deposition
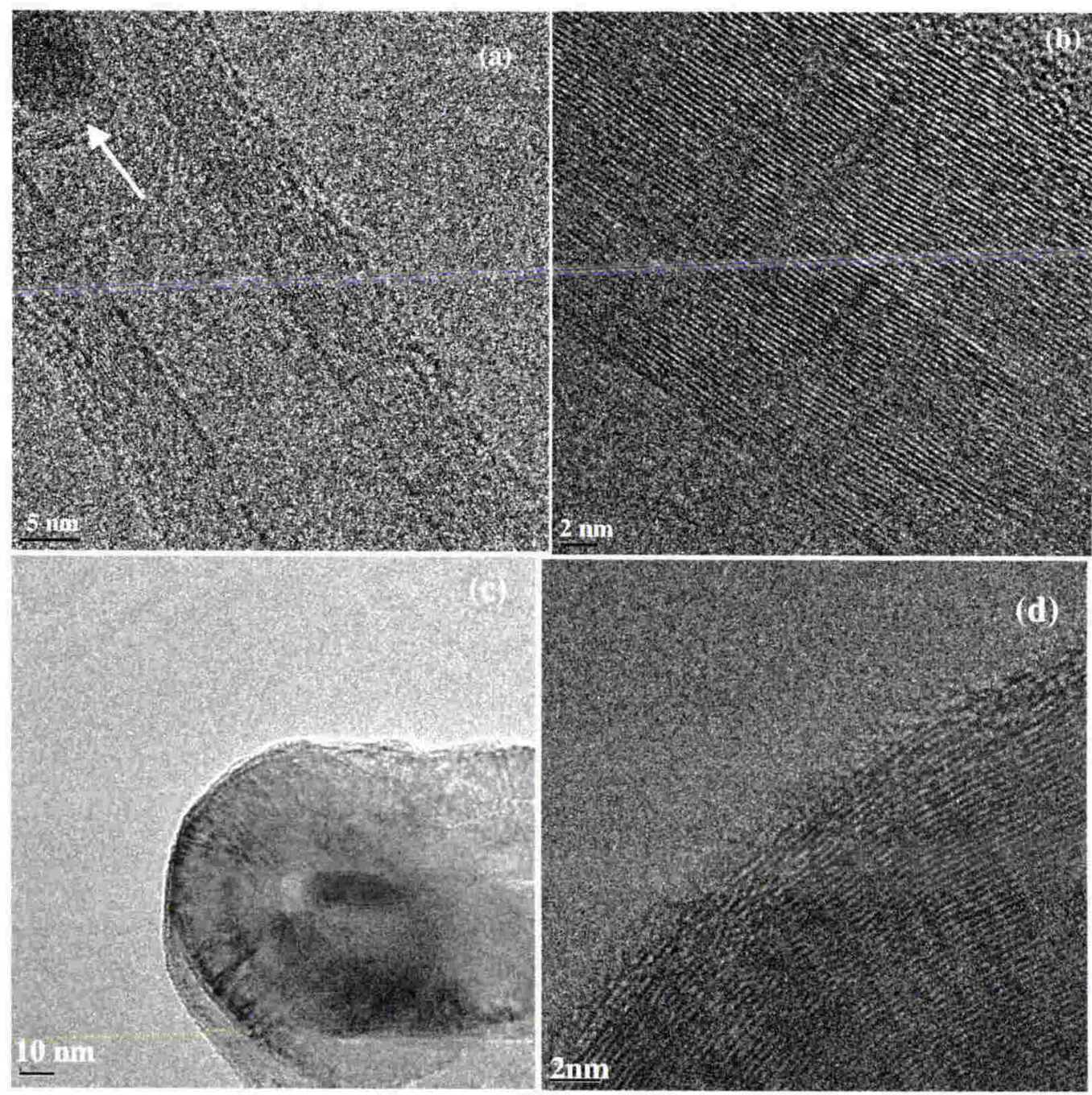

Figure 3-12 TEM images of (a) a concentric MWNT from ferrocene-derived catalysts under a high resolution. The white arrow indicates the impurities encapsulated inside the MWNT; (b) a MWNT section from FePC-derived catalysts under high resolution. The approximately $25 \mathrm{~nm}$ thick wall consists of 73 shells; (c) a MWNT tip under a low magnification; and (d) part of the outer wall of the MWNT tip in (c).

The CNT section captured in Figure 3-12 (b) is from FePC-derived catalysts and the HRTEM gives a well-resoluted view over the interlattice spacing, or the distances between adjacent shells along one of its two sidewall. There are 73 shells of graphitic lattices inside the wall of this CNT, of which the sidewall is about $25 \mathrm{~nm}$ thick. 
Figure 3-12 (d) gives an enlarged view over the edge of a CNT tip from ferrocenederived catalysts, as seen in Figure 3-12 (c). The image in (d) tells us that even though the lattices in the CNT tip bend to meet the enclosure of the tube, the inter-lattice spacing remains $0.34 \mathrm{~nm}$.

\subsubsection{EDX characterization of carbon nanotubes produced from ferrocene-derived and FePC-derived catalysts}

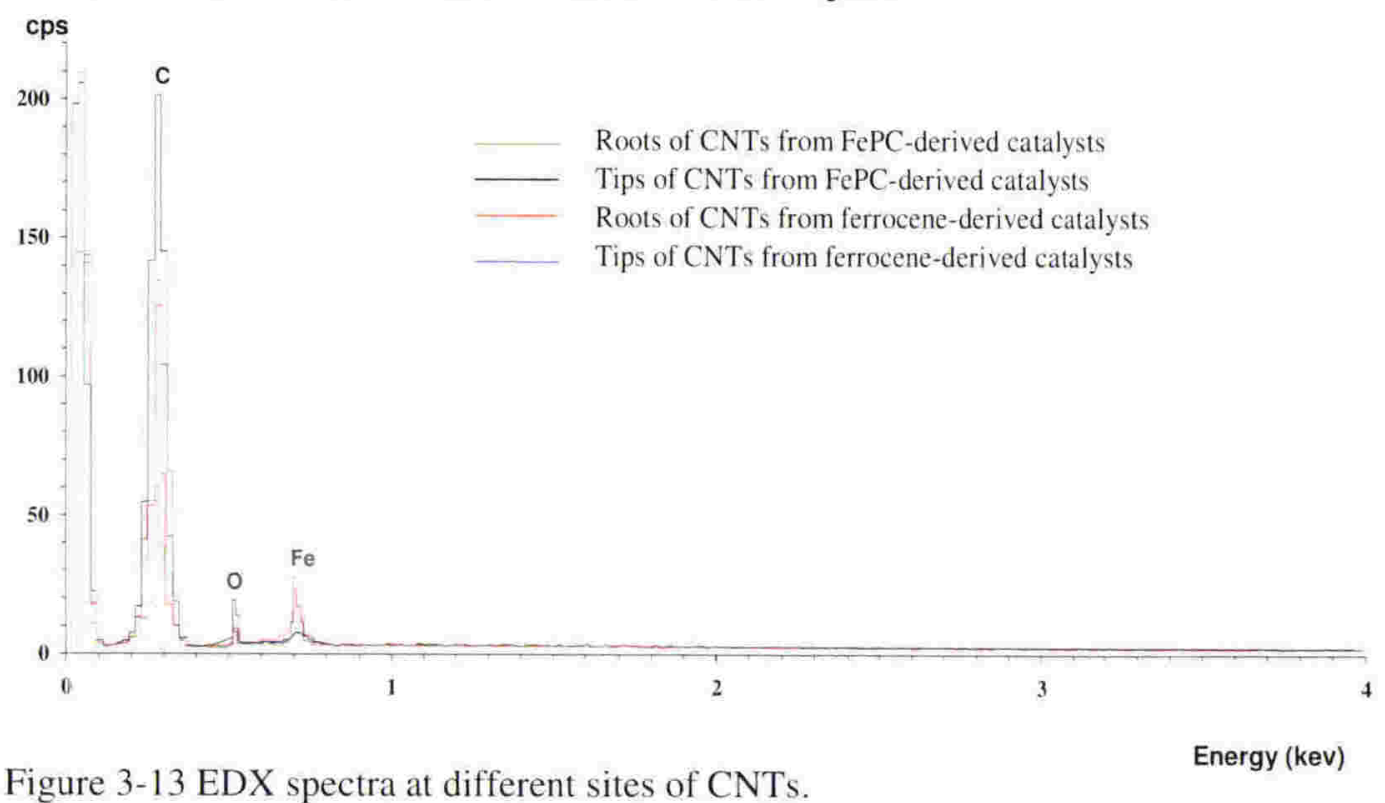

Energy Dispersive X-ray analysis (EDX analysis) is a technique used for identifying the elemental composition of the specimen of an area of interest. The EDX analysis system works as an integrated feature of a SEM. During EDX Analysis, the specimen is bombarded with an electron beam inside the SEM. The bombarding electrons collide with the specimen atoms' own electrons, knocking some of them off in the process. A position vacated by an ejected inner shell electron is eventually occupied by a higherenergy electron from an outer shell. To be able to do so, however, the transferring outer electron must give up some of its energy by emitting an X-ray. 
Chapter 3 Synthesis and Characterization of Multi-walled Carbon Nanotubes by Chemical Vapour Deposition

The amount of energy released by the transferring electron depends on which shell it is transferring from, as well as which shell it is transferring to. Furthermore, the atom of every element releases X-rays with unique amount of energy during the transferring process. Thus, by measuring the amount of energy present in the X-rays being released by a specimen during electron beam bombardment, the identity of the atom from which the X-ray was emitted can be established. Therefore, EDX spectrum gives the information on elemental atoms both qualitatively and quantitatively.

The EDX spectra, which were taken at different parts of the CNTs, as mentioned in the experimental, gave characteristic peaks for the carbon, iron, and oxygen elements, as shown in Figure 3-13 and the detailed data is listed in Table 3.1.

Table 3.1 Relative EDX peak intensity

\begin{tabular}{|c|c|c|c|c|}
\hline \multicolumn{2}{|c|}{} & \multicolumn{3}{c|}{ Counts of atom numbers from EDX } \\
\cline { 3 - 5 } \multicolumn{2}{|c|}{ Sample } & Carbon & Oxygen & Iron \\
\hline \multirow{2}{*}{$\begin{array}{c}\text { CNTs from } \\
\text { FePC }\end{array}$} & Roots & 57 & 7 & 8 \\
\cline { 2 - 5 } & Tips & 201 & 17 & 3 \\
\hline CNTs from & Roots & 113 & 7 & 19 \\
\cline { 2 - 5 } ferrocene & Tips & 122 & 7 & 24 \\
\hline
\end{tabular}

The carbon peak and the iron peak belong to the carbon contents in the sample and the particles derived from catalysts, respectively. The oxygen peak can be attributed to the $\mathrm{O}_{2}$ or $\mathrm{H}_{2} \mathrm{O}$, absorbed by the CNTs from the air. It should also be realized that there are no visible nitrogen peaks in the spectra either in CNTs from FePC or ferrocene. The absence of nitrogen from the CNT samples reflects two facts: first, there is no absorption of 
Chapter 3 Synthesis and Characterization of Multi-walled Carbon Nanotubes by Chemical Vapour Deposition

nitrogen from the air by the CNTs; second, the nitrogen atoms in FePC, do not participate in the formation of CNT structures.

The different parts of CNTs on which the EDX were taken include the roots, the sidewalls and the tips. The elemental analysis gave us some further characterizations on the spots detected. The data, which EDX analysis gives, is about the counts of atoms of different elements on a fixed area of the sample. By comparing the quantitative ratio between the numbers of iron and carbon atoms, one can tell the differences between samples from precursors and the differences between different regional parts of the same sample.

By comparing the counts of the elementary atom numbers of the CNT samples from different sources, as seen in EDX data in Table 3.1, we can draw some important information about their contents.

(1) There are large differences between the tips and roots in CNTs from FePCderived catalysts in the contents of iron and carbon while there is no significant difference in this aspect for CNTs from ferrocene-derived catalysts. This suggests that different mechanisms might occur during the growth of the two CNTs;

(2) Oxygen is absorbed by CNTs and has bigger content in the tips of the CNTs from FePC-derived CNTs. This might illustrate that the absorption of oxygen by CNTs more depends on quantity of carbon rather than the geometrical factor;

(3) CNTs from FePC contain less content of iron than their counterparts. This further supports the observation from EM images.

The EDX investigation is coupled with SEM and taken at a micrometer scale. In order to investigate the contents of the bulk samples, TGA is employed as described in the next section. 


\subsubsection{TGA investigation of carbon nanotubes produced from ferrocene- derived and FePC-derived catalysts}
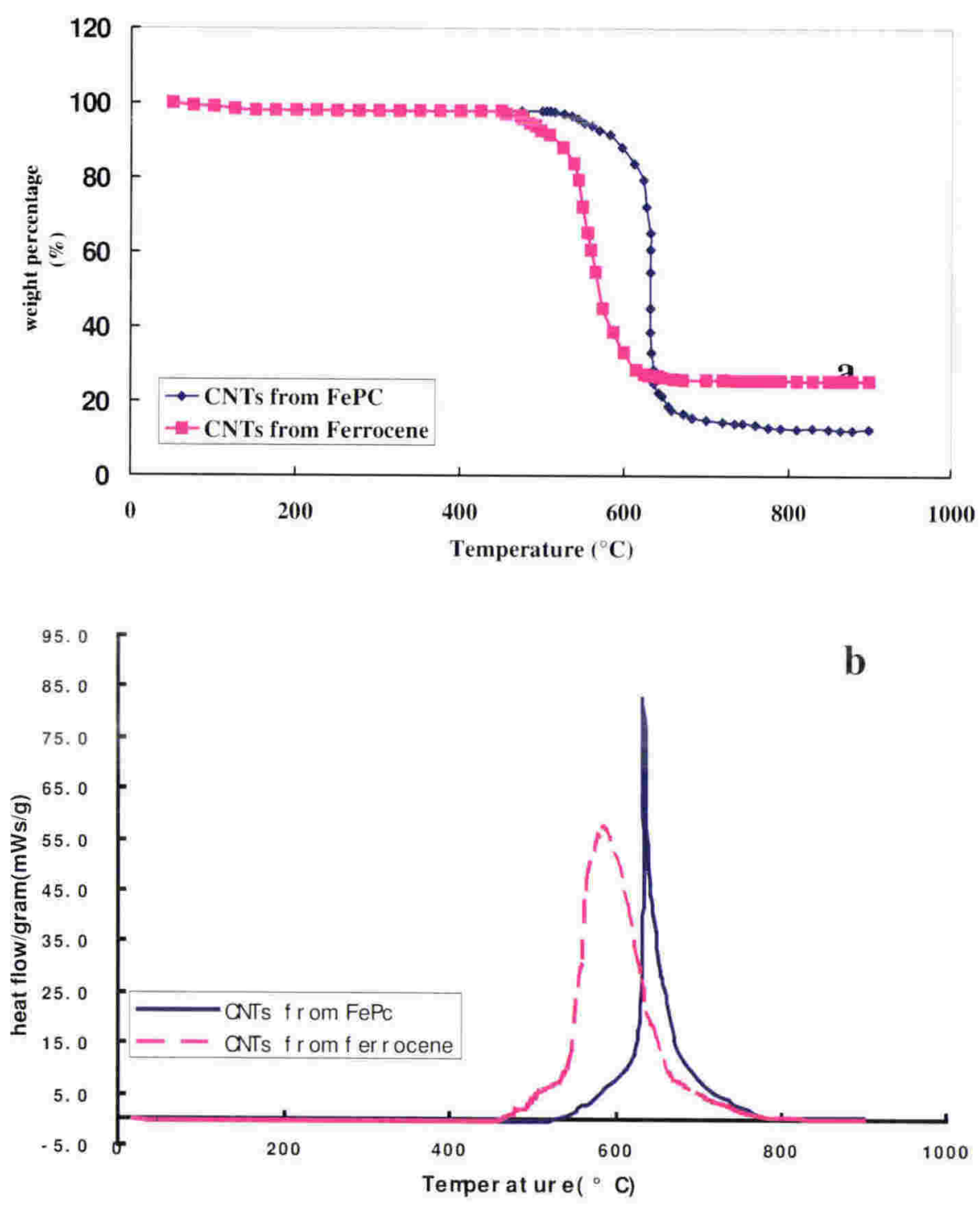

Figure 3-14 Thermogravimetric analysis of CNTs samples from ferrocene and FePC, ramped from $20-900 \mathrm{C}$ at $20{ }^{\circ} \mathrm{C}$ per minute under $20 \mathrm{sccm}$ flowing air. (a) Plots of weight percentage versus temperature. (b) Plots of heat flow per gram versus temperature.

In the thermogravimetric analysis characterization, an amount (2.0 to $5.0 \mathrm{mg}$ ) of CNT samples was burnt in the air. TGA gives the information on the weight loss and heat flow 
Chapter 3 Synthesis and Characterization of Multi-walled Carbon Nanotubes by Chemical Vapour Deposition

versus rising temperature for the CNTs grown from ferrocene and FePC during their oxidation in a hot air flow with the temperature ramped from room temperature to $900^{\circ} \mathrm{C}$. As seen in Figure 3-14 (a), both of the samples lost approximately $0.5 \%$ of their weight from 20 to $150{ }^{\circ} \mathrm{C}$. This weight loss can be attributed to evaporated $\mathrm{H}_{2} \mathrm{O}$, which had been absorbed in the CNTs. And the major weight loss ranges of CNTs from ferrocene and those from FePC are $473-635^{\circ} \mathrm{C}$ and $528-687^{\circ} \mathrm{C}$, respectively.

The thermostability of a sample depends very much on its structure. To account for the fact that the CNT samples from ferrocene start burning at a lower temperature than the CNTs from FePC, there are two factors that should be addressed. First, due to their closed and highly crystalline structure, CNTs are more chemically stable than amorphous carbon upon oxidation. The amorphous carbon in samples is responsible for the lower ignition temperature. Second, the defects along the walls of the CNTs are less chemically stable than the parts of CNTs without defects, so they are more vulnerable to oxygen at a lower temperature. After amorphous carbon and defects are oxidized, more dangling carbon bonds are left around the sites of the CNTs where the defects were attacked and to which amorphous carbon is adjacent. These dangling bonds are less chemically stable to oxidation than the carbon bonds in structurally perfect tubes. Therefore, amorphous carbon and defects lower the burning temperature range of the CNTs.

As seen from Figure 3-14 (b), the oxidation of CNT samples in air is an exothermal process. To make a better comparison between the two samples, the heat flow was replotted in $\mathrm{mW} \cdot \mathrm{g}^{-1}$ of sample. In these heat-flow plots, the peak temperatures of heat flow correspond to the steepest drop in weight loss from Figure 3-14 (a). They represent the temperatures at which the biggest weight portions of the CNTs were consumed in the 
Chapter 3 Synthesis and Characterization of Multi-walled Carbon Nanotubes by Chemical Vapour Deposition

burning process of as-grown CNTs. The peaks of heat release during burning are $587^{\circ} \mathrm{C}$ for CNTs from ferrocene and $633^{\circ} \mathrm{C}$ for CNTs from FePC. The former peak is broader than the latter one and that means the CNT samples from FePC are more uniform in their stability and structures.

The remaining material after burning at $900{ }^{\circ} \mathrm{C}$ is in a reddish colour. These residual materials are most likely to be $\mathrm{Fe}_{2} \mathrm{O}_{3}$. The mass ratios of the residuals to the original weight of the CNTs from ferrocene and FePC are $25.5 \%$ and $12.5 \%$, respectively. The molar ratios of $\mathrm{Fe} / \mathrm{C}$ for ferrocene-derived $\mathrm{CNTs}$ and $\mathrm{FePC}$-derived $\mathrm{CNTs}$ are approximately as $1 / 18$ and $1 / 45$, respectively.

It can be interpreted from the data shown above that, in a bulk scale, the samples from FePC contain more CNTs than those from ferrocene. They possess fewer impurities of iron, which can endure high temperature in the air, and fewer amorphous carbon, which burns in air, than the CNTs from ferrocene. The differences between the two samples from different catalysts can also be associated with the observations from EM images where there are fewer structural defects in the CNT structures from FePC.

\subsubsection{Raman spectroscopy of carbon nanotubes}

\subsubsection{Raman spectroscopy of SWNTs}

The Raman spectrum of a SWNT sample is shown in Figure 3-15. As was discussed in the previous chapter, several characteristic peaks are present in the figure. The modes of the other peaks observed in the spectra in Figure 3-15 are listed in Table 3.2. 
Chapter 3 Synthesis and Characterization of Multi-walled Carbon Nanotubes by Chemical Vapour Deposition

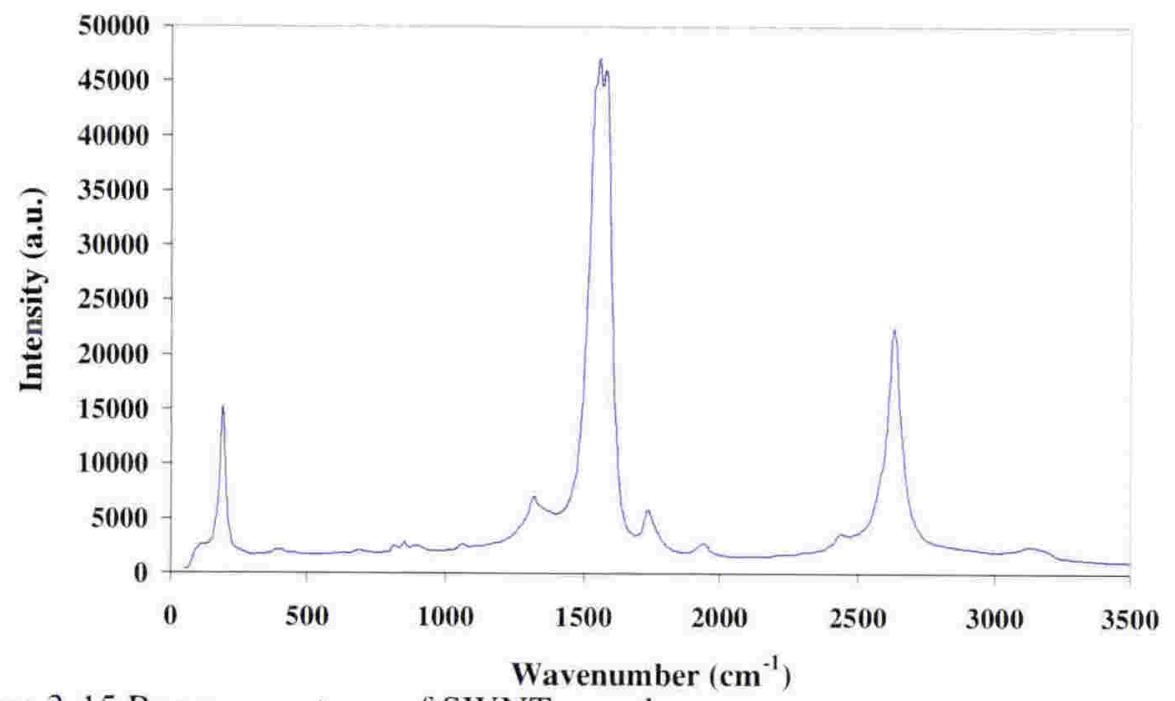

Figure 3-15 Raman spectrum of SWNT samples

As discussed in Chapter 1, the relation between diameter of the SWNT and RBM frequency is given by equation (1-7),

$$
\omega_{\mathrm{RBM}}=\mathrm{C}_{1} / \mathrm{d}_{\mathrm{t}}+\mathrm{C}_{2} \text {, }
$$

where $\mathrm{C}_{1}=223.5 \mathrm{~nm} \mathrm{~cm}^{-1}$ and $\mathrm{C}_{2}=12.5 \mathrm{~cm}^{-1}$.

Therefore, the diameter of the SWNT can be calculated from

$$
\mathrm{d}_{\mathrm{t}}=\mathrm{C}_{1} /\left(\omega_{\mathrm{RBM}}-\mathrm{C}_{2}\right)
$$

A strongly resonated RBM mode is observed at $191 \mathrm{~cm}^{-1}$, the corresponding diameter of this SWNT is approximately $1.25 \mathrm{~nm}$.

As listed in Table 3.2, the other Raman peaks of the SWNT spectrum in Figure 3-15 can be assigned as follows:

The weak peak at $1326 \mathrm{~cm}^{-1}$ is assigned to fewer disordered carbon structures in the sample. The $\mathrm{G}$ band consists of $\omega_{G}^{-}$and $\omega_{G}^{+}, 1555 \mathrm{~cm}^{-1}$ and $1582 \mathrm{~cm}^{-1}$ peaks, which are assigned to vibrations along the circumferential direction and along the CNT axis, respectively. $1745 \mathrm{~cm}^{-1}$ is a combination mode, which is RBM mode plus $\mathrm{G}$ band ( $\omega_{G}^{-}$in this case). And $2660 \mathrm{~cm}^{-1}$ is the second order peak corresponding to the D band. 
Chapter 3 Synthesis and Characterization of Multi-walled Carbon Nanotubes by Chemical Vapour Deposition

Table 3.2 First and second-order bands of SWNTs in Raman spectra of Figure 3-15

\begin{tabular}{l|l}
\hline Wavenumber $\left(\mathrm{cm}^{-1}\right)$ & Mode \\
\hline 191 & $\omega_{\text {RBM }}$, RBM, 1st order \\
1326 & $\omega_{\mathrm{D}}, \mathrm{D}$ band, 1st order \\
1555 & $\omega_{G}^{-}, \mathrm{G}$ band, 1st order \\
1582 & $\omega_{\mathrm{G}}^{+}, \mathrm{G}$ band, 1st order \\
1745 & $\mathrm{RBM}+\omega_{G}^{-}$, combination mode \\
2660 & $2 \mathrm{D}$ band, 2nd order \\
\hline
\end{tabular}

\subsubsection{Raman spectroscopy of MWNTs}

Raman spectroscopy was employed to investigate aligned MWNTs. The Raman spectroscopy of MWNTs was reviewed in Chapter 1 and the spectra of aligned FePCderived MWNTs are shown in Figures 3-16 and 3-17, and their characteristics are summarized as:

\section{First-order peaks}

There are three pronounced peaks which need to be addressed:

(1) The peaks at $1582 \mathrm{~cm}^{-1}$ and $1591 \mathrm{~cm}^{-1}, \omega_{G}^{-}$and $\omega_{G}^{+}$[22], are attributed to the graphitic form of the CNTs as the $\mathrm{E}_{2 \mathrm{~g}}$ mode and are assigned to the vibrations along circumference and axis of CNTs, respectively. The average of the above two peaks, approximately $1587 \mathrm{~cm}^{-1}$, as discussed in Chapter 1 , is the typical feature of 2D graphite, which only has one type of vibration and thus one $\mathrm{G}$ band peak. Compared with the two counterparts in the spectrum of SWNTs, the gap 
Chapter 3 Synthesis and Characterization of Multi-walled Carbon Nanotubes by Chemical Vapour Deposition

between $\omega_{G}^{-}$and $\omega_{G}^{+}$, is much smaller because MWNTs have less curvatures in their shells [12] and are closer to a 2D graphite structure than SWNTs.

(2) The peak at $1333 \mathrm{~cm}^{-1}[16,22], \omega_{\mathrm{D}}$, according to Dresselhaus et al. [22], arises from a symmetry-lowering effect, due to defects or CNT caps, bending of the CNT, and the presence of carbon nanoparticles and amorphous carbon. In another word, there might be some disorder carbonaceous forms in the sample, even though SEM and TEM images show the sample is quite clean and uniform.

(3) The peak at $1618 \mathrm{~cm}^{-1}$, is only present in Raman spectra of MWNTs and not observed in the SWNT spectra in Figure 3-15. It is related with the maximum in the graphene 2D phonon [22].

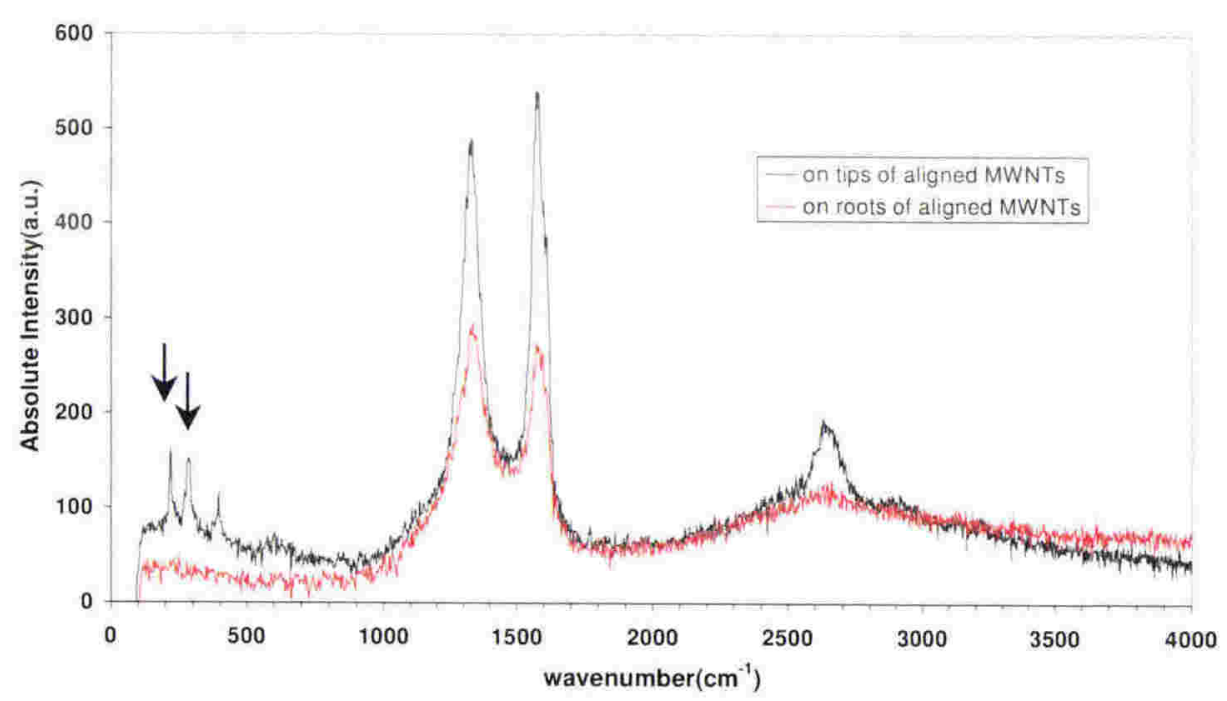

Figure 3-16 Raman spectroscopy on an aligned film of MWNTs from FePC. Laser spots on the tips of and on the roots of the CNT film. The two solid arrows indicate the two peaks in RBM mode.

\section{Second-order peaks}

The second order Raman spectra show broad peaks at $2643 \mathrm{~cm}^{-1}$ and $2667 \mathrm{~cm}^{-1}$, G' band, for which it has been suggested by the reference [13] that they can be attributed to the 
Chapter 3 Synthesis and Characterization of Multi-walled Carbon Nanotubes by Chemical Vapour Deposition

"disorder within graphite domains". In the Raman spectra shown in Figure 3-16, this peak can be attributed to $2 \omega_{\mathrm{D}}$.

When estimating the defect content in the as-grown CNT samples, the D-mode intensity is usually normalized with respect to the intensity of the G-mode at around $1600 \mathrm{~cm}^{-1}$ to yield a relative intensity of $\mathrm{D}$ band over $\mathrm{G}$ band. This ratio indicates both the defect content inside the regular graphitic structures of CNTs and the amorphous carbon content in the sample. A lower relative intensity of D band over $\mathrm{G}$ band corresponds to a better crystallisation in the sample.

As seen from Figure 3-16, the Raman signal from the roots of the CNTs is weaker than that from the tips of nantoubes, this is due to less crystalline graphitical structures, which were seldom observed in the TEM image (Figure 3-9 (c)) of the FePC-derived CNT roots. Also shown in Figure 3-16, RBM Peaks, which are indicated by the two solid arrows in the figure, the typical characteristic of Raman spectra of SWNT and MWNTs with low diameters [2], appeared on the tips of FePC-derived CNTs. Even though none of SWNTs have been identified from the numerous TEM images of FePC-derived CNTs, the possibility of the existence of MWNTs with low diameters or even SWNTs in FePCderived CNT samples should not be excluded. On the contrary, as shown in Figure 3-17, the sample from ferrocene-derived catalysts does not contain RBM-active CNTs.

Figure 3-17 compares Raman spectra of MWNTs from ferrocene-derived catalysts and FePC-derived catalysts. The relative intensities of D band versus $\mathrm{G}$ band in the spectra indicate that, compared with their ferrocene-derived counterparts, FePC-MWNTs contain more regular graphitic nanostructures and relatively fewer defects and carbon-related impurities. That is in consistence with the observations from TEM and SEM. 
Chapter 3 Synthesis and Characterization of Multi-walled Carbon Nanotubes by Chemical Vapour Deposition

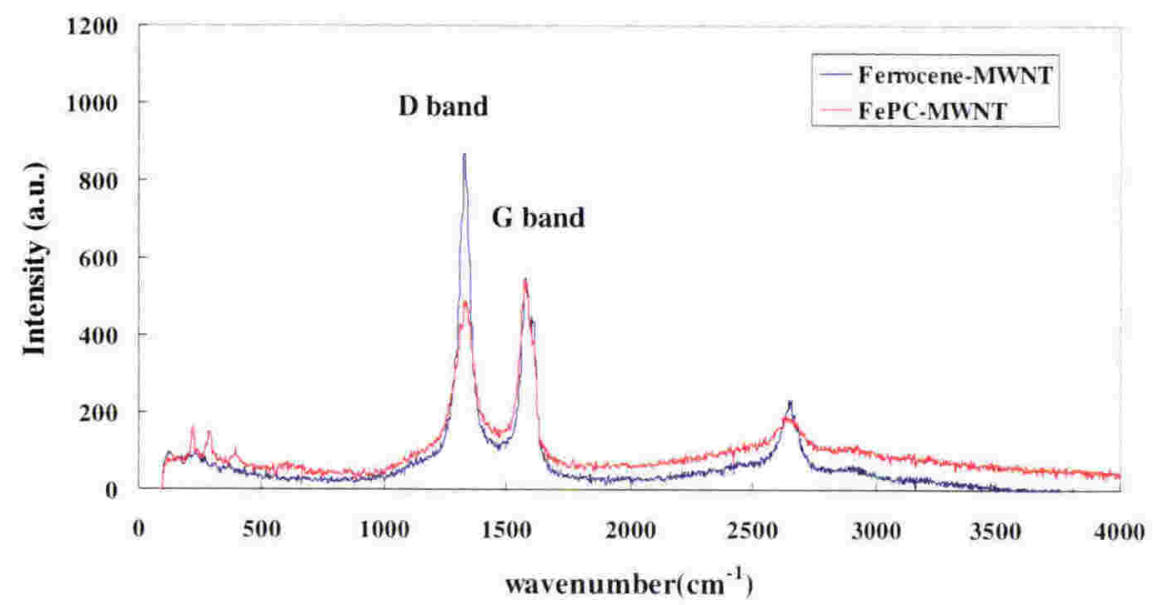

Figure 3-17 Raman spectra of MWNTs from ferrocene-derived and FePC-derived catalysts.

\subsubsection{Growth mechanism of carbon nanotubes}

The growth mechanism of carbon nanotubes in CVD has been extensively studied [1719]. Here the discussions are focused on three subtopics:

1. Why do large amounts of defects and coiled structures appear in carbon nanotubes grown from $\mathrm{Fe}\left(\mathrm{NO}_{3}\right)_{3}$-derived nanoparticles? 2. How is the alignment of CNTs constructed under the thermal decomposition route? 3 . Why are there some differences between CNT samples from ferrocene-derived and FePC-derived catalysts?

\subsubsection{Investigation of growing carbon nanotubes from $\mathrm{Fe}\left(\mathrm{NO}_{3}\right)_{3}$-derived nanoparticles}

It can be observed that multi-walled carbon nanotubes grown from $\mathrm{Fe}\left(\mathrm{NO}_{3}\right)_{3}$-derived nanoparticles contain large amounts of defects and coils, as illustrated earlier in Figures 3-2, 3-3 and 3-11. To understand this, it will be useful to start with how a defect in a CNT is formed in an atomic scale. CNTs remain in perfectly straight forms as long as the carbon atoms are all in hexagons. However, there are some heptagons and pentagons in tubular sections and these sites were observed as defects in TEM images. As shown in 
Chapter 3 Synthesis and Characterization of Multi-walled Carbon Nanotubes by Chemical Vapour Deposition

Figure 3-18, an "elbow-like bend" [8] is one of the defects which can be found in CNT structures. Heptagons and pentagons are structurally essential for such a bend. The heptagons and pentagons not only exist in defects and curves along the CNTs, but they are also necessary geometric parts in the closure of CNT tips. Topological defects have been introduced in CNT structures to achieve electrical contacts at a molecular level [7].

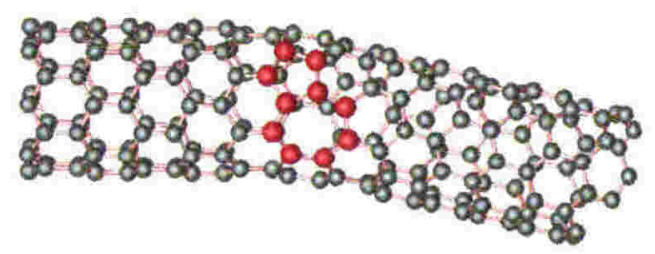

Figure 3-18 "Elbow-like bend" (Chico, et al. [6]) between two pieces of straight CNTs $(8,0)$ and $(7,1)$. The carbon atoms in red form into a pentagon and a heptagon while most of the other carbon atoms in blue form into hexagons.

The formation of defects and coils can be attributed to the irregularity of the catalyst nanoparticles. The large variety of the nanoparticles lead to uneven distribution [1] of the growth rate at different growing spots around the circumferences of the CNTs during the growth process. The consequence is that CNTs are grown in a great variety of diameters and pointing to different directions. These factors as well as the entanglement of CNTs result in CNTs with defects and coils in the process of their growth.

\subsubsection{Mechanism of alignment of carbon nanotubes by CVD}

The growth of aligned carbon nanotubes by CVD can be illustrated as follows: The sublimed precursors (ferrocene or FePC) are carried by argon into the pyrolysis chamber, where the precursors are decomposed into Fe nanoparticles. These particles interact with the quartz glass plate and grow into nucleation sites. The decomposed carbon atoms or species from hydrocarbon diffuse and saturate from Fe clusters and grow into capped 
Chapter 3 Synthesis and Characterization of Multi-walled Carbon Nanotubes by Chemical Vapour Deposition

graphitic shells. Then more carbon species either diffuse into the metal particles or form into the graphitic walls, which form into new caps or open tips and extend into tubes. The high density of active sites results in the "overcrowding effect" [23] onto each CNT during the growing process. And the inter-CNT van der Waals forces attach CNTs together and enable carbon radicals to grow vertically into aligned carbon nanotubes films.

Combining the results from TEM and EDX, the growth mechanism of the CNTs from FePC-derived catalysts is more inclined to the base-growth mechanism, as in the model suggested by Lee [17]. Most of the catalysts stay inside the roots of the CNTs. It can be depicted that, during the growth of FePC- derived CNT forests, the saturation rate of carbon atoms inside the metal-carbon alloy is much faster than the dissipation rate, and the over-saturated carbon atoms deposit along the up growth direction of the CNTs.

\subsubsection{Investigation on the differences between carbon nanotubes from ferrocene- derived and FePC-derived catalysts}

The differences between CNT samples from ferrocene-derived and FePC-derived catalysts have been deduced from the characterization described in the earlier sections. As mentioned before, it is postulated that the former CNTs were grown under both the tip- and base-growth mechanisms, whilst the latter ones were grown via a base-growth mechanism.

Besides the disparity between their growing mechanisms, their differences in iron contents, CNT morphology and thermostability can be explained by two factors. First, compared with ferrocene (molar ratio, $\mathrm{Fe} / \mathrm{C}: 1 / 10$ ), the ratio of $\mathrm{Fe}$ content in $\mathrm{FePC}$ (molar ration, $\mathrm{Fe} / \mathrm{C}: 1 / 32$ ) is much less. It means that less $\mathrm{Fe}$ content from $\mathrm{FePC}$ is involved in 
Chapter 3 Synthesis and Characterization of Multi-walled Carbon Nanotubes by Chemical Vapour Deposition

the synthesis of CNTs than from ferrocene. Second, FePC sublimes at a much higher temperature than ferrocene and cracks into more uniform catalytic species. It starts to boil at $478{ }^{\circ} \mathrm{C}$ whilst ferrocene sublimes at $175^{\circ} \mathrm{C}$. In another word, under a ramping temperature, ferrocene is more volatile than its counterpart. It means that the FePC has a smaller range of sublimation temperatures than ferrocene during the process in which catalysts deposit on the growth substrate. Therefore the catalytic species and carbon species, which were cracked from FePC, might be less versatile than those from ferrocene. We would also like to associate these differences in the physical properties of the precursors with the different geometry of the catalyst particles on the growth substrates and as a result, a different quality of the CNT samples.

These two factors of the precursors might contribute to the facts that catalyst particles derived from FePC are more uniform in their sizes and more likely to transform carbon species into CNTs than those from ferrocene.

The work discussed in this chapter demonstrated that CVD can be used as an efficient method for the synthesis of MWNTs. Characterizations on CNTs revealed the differences in morphologies and thermostabilities of CNTs, which are attributed to the differences in their growth mechanisms and the volatilities of their precursors. In the next chapter, attentions will be brought to the after-synthesis treatment of MWNTs. 
Chapter 3 Synthesis and Characterization of Multi-walled Carbon Nanotubes by Chemical Vapour Deposition

\section{Reference:}

1. S. Amelinckx, X. B. Zhang, D. Bernaerts, X. F. Zhang, V. Ivanov, and J. B. Nagy (1994), Science, 265: p. 635-639.

2. J. M. Benoit, J. P. Buisson, O. Chauvet, C. Godon, and S. Lefrant (2002), arXiv:cond-mat, v1: p. 0205031.

3. L. P. Biro, G. Molnar, I. Szabo, Z. Vertesy, Z. E. Horvath, J. Gyulai, Z. Konya, P. Piedigrosso, A. Fonseca, J. B. Nagy, and P. A. Thiry (2000), Applied Physics Letters, 76: p. 706-708.

4. A. M. Cassell, J. A. Raymakers, J. Kong, and H. Dai (1999), Journal of Physical Chemistry B, 103: p. 6484-6492.

5. H. M. Cheng, G. S. F. Li, H. Y. Pan, L. L. He, X. Sun, and M. S. Dresselhaus (1998), Applied Physics Letters, 72: p. 3282-3284.

6. L. Chico, V. H. Crespi, L. X. Benedict, S. G. Louie, and M. L. Cohen (1996), Physical Review Letters, 76: p. 971-974.

7. L. Chico and W. Jaskolski (2004), Physical Review B, 69: p. 085406-1.

8. P. J. F. Harris (1999), in book. Carbon Nanotubes and Related Structures Cambridge: Cambridge University Press

9. S. Huang, L. Dai, and A. W. H. Mau (1999), Journal of Material Chemistry, 9: p. 1221-1222.

10. S. Huang, L. Dai, and A. W. H. Mau (1999), Journal of Physical Chemistry, 103: p. $4223-4227$.

11. S. Huang, L. Dai, and A. W. H. Mau (2002), Advanced Materials, 14: p. 11401143 .

12. A. Jorio, G. Dresselhaus, M. S. Dresselhaus, M. Souza, M. S. S. Dantas, M. A. Pimenta, A. M. Rao, R. Saito, C. Liu, and H. M. Cheng (2000), Physical Review Letters, 85: p. 2617-2620.

13. R. Kamalakaran, M. Terrones, T. Seeger, P. Kohler-Redich, M. Ruhle, Y. A. Kim, T. Hayashi, and Endo (2000), Applied Physics Letters, 77: p. 3385-3387.

14. N. T. Kemp (2000), Electronic transport in the conducting polymer polypyrrole, PHD Thesis, Victoria Univeristy of Wellington, New Zealand 
Chapter 3 Synthesis and Characterization of Multi-walled Carbon Nanotubes by Chemical Vapour Deposition

15. H. Kind, J.-M. Bonard, C. Emmenegger, L.-O. Nilsson, K. Heradi, E. MailardSchaller, L. Schapbach, L. Forro, and K. Kern (1999), Advanced Materials, 11: p. 1285-1289.

16. C. J. Lee, D. W. Kim, T. J. Lee, Y. C. Choi, Y. S. Park, W. S. Kim, Y. H. Lee, W. B. Choi, N. S. Lee, J. M. Kim, Y. G. Choi, and S. C. Yu (1999), Applied Physics Letters, 75: p. 1721-1723.

17. C. J. Lee and J. Park (2000), Applied Physics Letters, 77: p. 3397-3399.

18. W. Z. Li, J. G. Wen, and Z. F. Ren (2002), Applied Physics A: Materials Science \& Processing, 74: p. 397-402.

19. O. A. Louchev, Y. Sato, and H. Kanda (2002), Applied Physics Letters, 80: p. $2752-2754$

20. C. N. R. Rao, G. U. Kulkarni, A. Govindaraj, B. C. Satishkumar, and P. J. Thomas (2000), Pure Applied Chemistry, 72: p. 21-33.

21. C. N. R. Rao, R. Sen, B. C. Satishkumar, and A. Govindaraj (1998), Chemical Communication: p. 1525-1526.

22. R. Saito, G. Dresselhaus, and M. S. Dresselhaus (1998), in book. Physical properties of carbon nanotubes London: Imperial College Press

23. M. Terrones, N. Grobert, J. Olivares, J. P. Zhang, H. Terrones, K. Kordatos, W. K. Hsu, J. P. Hare, P. D. Townsend, K. Prassides, A. K. Cheetham, H. W. Kroto, and D. R. M. Walton (1997), Nature, 388: p. 52-55.

24. Z. Zhang, B. Wei, J. W. Ward, R. Vajtai, G. Ramanath, and P. M. Ajayan (2001), Advanced Materials, 13: p. 1767-1770.

25. Z. J. Zhang, B. Q. Wei, G. Ramanath, and P. M. Ajayan (2000), Applied Physics Letters, 77: p. 3764-3766. 


\section{Part II}

\section{Post-synthesis Treatment on Carbon Nanotubes by Dielectrophoresis}




\section{Chapter 4 \\ Post-synthesis Treatment of Carbon Nanotubes}

The previous part of this thesis focused on our synthesis and characterisation of as-grown multi-walled carbon nanotubes by CVD from different precursors. However, for some characterisations and most of applications purposes, as-grown CNTs need to be treated before use. In the second part of this thesis, attentions will be given to the post-synthesis treatment of carbon nanotubes and the background chemistry behind these treatment methods will be introduced.

Treatment of CNTs after synthesis has been under scrutiny since the discovery of CNTs in 1991. It became an interesting topic not only because of the application necessity but also because investigations into the treatment mechanisms reveal further information about the intrinsic chemical and physical properties of CNTs. Several aspects involved with post-synthesis treatment of carbon nanotubes: purification, modification, separation and manipulation are under scope of this chapter. Various methods to achieve these goals are to be addressed here. The mechanisms of these treatments will also be reviewed.

\subsection{Purification}

As-grown CNTs contain impurities, which may deteriorate their properties. These impurities include amorphous carbon, carbon nanoparticles and metal particles, which are derived from the catalysts. Proper ways of purification are required $[14,16,38,46,50]$ to remove these impurities before the intrinsic properties of CNTs can be studied and 
Chapter 4 Post-synthesis Treatment of Carbon Nanotubes

excellent performances of CNTs can be fully exploited. Different methods of purification have been reported, including burning [16] in $\mathrm{CO}_{2}$ and air, ultrasonically assisted filtration [38], oxidation by means of acid [14] or bromine [13], toluene extraction [14] and dielectrophoresis [50] (which will be discussed in details in Chapter 5).

\subsubsection{Oxidation of CNTs in $\mathrm{CO}_{2}$ and air}

Oxidation of CNTs in $\mathrm{CO}_{2}$ and air, or burning of CNTs, was first adopted as a purification method by Ebbesen [16] in 1994. This approach targets carbon nanoparticles, which are the main by-products of an arc-discharge process. These nanoparticles include small-sized ones like fullerenes and big-sized ones, such as carbon nano-onions (carbon particles with multi-layered spherical structures). In a burning process, carbon nanotubes are oxidized at a lower rate and are able to survive at a higher temperature than carbon nanoparticles because CNTs are structurally more stable (the thermostabilities of MWNTs have been studied in Chapter 3).

However, to produce pure CNTs, ninety-nine percent of the sample, including a considerable quantity of CNTs, had to be burnt off, as reported in reference [16]. Another disadvantage of this method is that burning of the samples leads to some unwanted opening and thinning $[1,47]$ on the walls of CNTs.

\subsubsection{Oxidation by liquid oxidants}

Liquid oxidants have also been used to purify CNT samples from a different route than the method mentioned above. The liquid-phase oxidation reaction is more effective than its gas-phase counterpart in removing metal oxide particles derived from catalytically grown CNTs, with which the main by-products are metallic impurities. Among these 
Chapter 4 Post-synthesis Treatment of Carbon Nanotubes

liquid oxidants, there are some strong reagents such as $\mathrm{KM}_{\mathrm{n}} \mathrm{O}_{4} / \mathrm{H}_{2} \mathrm{SO}_{4}$ [20], concentrated $\mathrm{HNO}_{3} / \mathrm{H}_{2} \mathrm{SO}_{4}$ and bromine [13] for multi-walled carbon nanotubes, and mild one such as a dilute $\mathrm{HNO}_{3}$ [14] solution for single-walled carbon nanotubes. To achieve a uniform result of purification, a mechanical stirring or a reflux is usually applied.

However, liquid oxidants may bring some damages onto nanotube structures and in particular they also make openings on CNTs because CNT tips are more vulnerable under oxidation. After being oxidized by these strong liquid reagents, hydroxyl and carboxyl groups are generated on the sites where carbon atoms on CNTs are attacked, and sometimes resulted in shortening of CNTs.

\subsubsection{Ultrasonically assisted filtration}

Smalley et al. first reported a purification method of ultrasonically assisted filtration [38]. This approach was based on size selection of membranes. In a typical experimental procedure of ultrasonically assisted filtration, single-walled CNTs-containing samples, which were produced by the laser-ablation method, were suspended in a surfactant solution, followed by filtering through a polymeric membrane with micron pore size. An ultrasonic horn has been employed in Smalley's report in order to achieve a good suspension of the sample and to ease blockings in the membrane pores. Most of amorphous carbon and metal particles in as-grown samples were filtered off and CNTs were obtained from the filtrate.

\subsubsection{Reflux and extraction by organic solvents}

CNTs have much less solubility than metal particles and fullerenes in toluene. In the reflux and Soxhlet extraction method of CNT samples in toluene from reference [46], 
Chapter 4 Post-synthesis Treatment of Carbon Nanotubes

metal particles and fullerene impurities were dissolved and the undissolved CNTs were left as the residue. This method is particularly effective on samples from arc-discharge and CVD synthesis.

\subsection{Modification}

CNTs can be modified either physically or chemically to meet the specific needs for applications. On one hand, carbon nanotubes are structurally stable and their fundamental properties do not change after a mild modification; on the other hand, some changes of their structures and properties enhance the properties of CNTs and broaden their application potentials.

\subsubsection{Structural modification}

As reviewed in Chapter 1, CNTs have very large potentials in MEMS and NEMS applications. However, they need to be opened and truncated for some uses in these aspects. As-grown CNTs are usually of microns long and in bundles, as a result, they are not desirable to fit into some nanometre-sale electronic and mechanical devices and need to be cut [32] and isolated [24] into shorter and individual sections before use.

Also, open tips are necessary for capillary uses [1, 43, 47] and hydrogen storage [39]. Thus an opening of the enclosed ends of CNTs is required.

Cutting and opening can be achieved by burning CNTs in air, electrochemical etching [39], ball milling, ultrsonication [52] or refluxing CNTs in concentrated $\mathrm{HNO}_{3}$. So openended CNTs can be considered as by-products of a purification process. It has been observed [47] that opening usually happens at the sites with the greatest curvature or on the tips. The curvatures, which have been described in Chapter 3 as holding high 
densities of defects, are less structurally stable than the straight compartments because carbon pentagons and heptagons, which are contained in curvatures, are energetically less stable than hexagons in straight tubules and therefore are more vulnerable to oxidations.

\subsubsection{Chemical modification}

CNTs without any modifications are extremely hydrophobic and not suitable for applications in which polar liquid media, such as biological uses [7], are involved due to the fact that CNTs are lack of solubility under pristine conditions. Therefore, either solvents $[2,3,11]$ and surfactants [8] are required or the CNTs need to be modified $[5,23$, 37, 49] and chemically functionalised [15, 31, 45]. And more importantly, chemical modification is expected to provide "chemical handles" [42] to overcome the difficulties in manipulating CNTs in producing CNT-based devices.

\section{Dispersion of CNTs in solvents and surfactant environments}

CNTs without modification are highly hydrophobic. Among reports, only single-walled CNTs have very slight solubilities in some solvents such as 1,2-dichlorobenzene, toluene, chloroform, dimethylsulphoxide (DMSO), N,N-dimethylformamide (DMF), Nmethylpyrolidone (NMP) and tetrahydrofuran (THF) before being properly modified. The reported solubilities of SWNTs are less than $100 \mathrm{mgL}^{-1}$ [2]. Moreover, in fact, a colloid suspension, rather than solution, of CNTs in a solvent, was obtained. To precisely describe the behaviour of CNTs in a liquid medium, the term "colloid solution" or "dispersion" is frequently used. 
According to colloid chemistry [34], dispersions are always in a metastable state. The stability of the dispersion is strongly dependent on the charges generated on the surface of the particles. The combination of an attractive van der Waals force and a repulsive electrostatic force forms the fundamental basis for an understanding of a colloid solution. The instability of colloid suspension arises from van der Waals forces between colloid particles. Van der Waals force is a ubiquitous force in nature and one of the main forces acting between molecules and is responsible for holding solids and liquids together. It is much weaker than the Coulombic force acting between ions.

A universally adopted method to achieve a stable and well-dispersed suspension is to use a surfactant [8], which provides repulsive forces between colloid molecules to achieve the dispersion of nanotube colloids. The uses of both of ionic surfactants, such as sodium dodecyl sulphate (SDS) [8], and non-ionic surfactants Triton X-100 [2] and polyvinylpirrolidone (PVP) [29], have been reported. It should be realized that dispersion of CNTs in a surfactant solution is a thermodynamic process and therefore it can be reversed.

Ultrasonication treatment, which is often used for TEM sample pre-treatment for CNTs, is usually coupled with a surfactant-assisted dispersion to provide mechanical energy to disintegrate the nanotube aggregates and to overcome the van der Waals forces between nanotube colloids.

\section{Chemical functionalisation}

Carbon atoms in CNTs are in $s p^{2}$ hybridisation and bind in a conjugated way. Different routes to achieve covalent functionalisation based upon the electronic structures of CNTs, have been raised: 
(1) Introducing carboxyl acid group (-COOH) [48] onto nanotube's outer walls, followed by coupling amines to this group.

(2) Addition of aryl group onto sidewalls of SWNTs using diazonium salts.

(3) Dipolar cyclo-addition of heterocyclic groups [7] or dichlorocarbene [22] onto sidewalls of CNTs.

The chemical modification of CNTs causes an increased solubility [5] and altered band gaps $[39,43]$ as well.

The reactivity of CNTs is strongly dependent on their local deformation, chemical doping, photo-excitation and local chemical environment [42].

Also, CNTs can be functionalised with polymers such as polyvinyl alcohol [31] in carbodiimide-activated esterification reactions to yield nanotube/polymer nanocomposites in which the interaction between CNTs and polymer chains is enhanced after the reaction. And as a result, the mechanical properties of the composite are improved.

By comparing between the effects of solvent/surfactant and chemical modification on CNTs, it can be concluded that solvent/surfactant treatment does not change the structure of CNTs and therefore the treatment is reversible. In the case of chemically modification of CNTs, by contrast, introducing chemical functional groups onto CNTs alters the electronic structures of the resulting tubes. To remove chemical functional groups from CNTs, a heat treatment is necessary. A high temperature introduces cross-linkings between sidewalls of CNTs [22] and reduces the dispersion ability of CNTs. As a result, chemical functionalisation method brings some irreversible changes to CNT structures. 


\subsection{Separating between semiconducting and metallic CNTs}

For electronic uses, semiconducting and metallic CNTs ought to be separated, or CNTs of specific chiralities need to be sorted $[4,27]$. There are some reported attempts with different selection mechanisms, which were based on the differences of structures and chemical properties between metallic and semiconducting CNTs. Among these methods, there are dielectrophoresis [26], selective physisorption [9] and DNA-assisted selection $[52,53]$

\subsubsection{Selective physisorption}

In a physisorption process, a substance absorbs species while there are no chemical bondings between them. For a semiconductor, physisorption changes its number of charge carriers and results in a shift in their Fermi level. It has been found [25] that doping by alkylamine, a $-\mathrm{NH}_{2}$ group on individual semiconducting SWNTs shifts the Fermi level of CNTs. Therefore, there is an affinity between semiconducting CNTs and the amine group. A bulk separation route was proposed by Charttopadhyay et al. as selectively destabilising the dispersion of metallic CNTs [9] in an octadecyl amine (ODA) environment to cause their precipitation while semiconducting CNTs were being kept dispersed in ODA.

\subsubsection{DNA-assisted sorting}

The unique sequenced structure of DNA has been used to carry out molecular recognition. An oligonucleotide sequence was reported to be able to self-assemble into highly-ordered structures around CNTs [52, 53], allowing improved separation between metallic and semiconducting CNTs as well as a diameter-dependent separation. 
In a DNA-assisted sorting process, the chemical coupling of CNTs with DNA was followed by anion exchange chromatography. The mechanism of this process was based on the fact that the interactions of semiconducting SWNT and metallic SWNT with DNA result in different net linear charge densities of their structures and lead to discrepancy in the binding strengths of metallic and semiconducting SWNTs with the anion exchange resin. Therefore sampling elution fractions of anion exchange chromatography at different retaining times achieves the separation of these two types of SWNTs from each other.

\subsection{Post-synthesis alignments and positioning}

Aligned CNTs have been synthesized from CVD, as described in Chapter 3. However, specific application may require alignment and/or re-positioning of CNTs after synthesis. This has been accomplished both mechanically and electrically. Assembling CNTs into desired places and shapes has also been required. The tiny sizes of CNTs retard any easy manipulation on them. Post-synthesis treatment and manipulation of carbon nanotubes still remain the major barriers [6] toward their large-scale applications. Among reports in these aspects, ion beam irradiation [35] and laser pruning [30] have been attempted to modulate the patterns and the structures of CNTs after synthesis. However, they are still not satisfactory cures to the hassles above.

Also, in order to gear CNTs from a laboratory research object to industrially applicable materials, appropriate ways of aligning, manoeuvring and positioning [51] CNTs in a bulk scale are required. Rheological forces [19], chemical self-assembly, magnetic field $[18,21]$ and electric field $[12,17,26,28,33,44,50]$ have been used to introduce 
alignment onto post-synthesis CNTs and with some of those methods, it has been possible to fabricate CNTs into preliminary devices.

\subsubsection{Rheological forces}

The earliest attempt of aligning CNTs into a certain direction after synthesis can be traced back to 1995 when W. A. de Heer at al. [19] reported a method of aligning arc chargesynthesized CNTs by mechanically pressing a piece of CNTs-coated ceramic filter onto a thin Teflon sheet. Measurements on the resistance and dielectric functions of the aligned samples along the perpendicular and parallel directions of the alignment indicated anisotropies in their electrical and optical properties, which were clear evidences of morphologic alignment of CNTs.

\subsubsection{Magnetic force}

According to de Heer et al. [10], CNTs are magnetically susceptible materials. As a result, alignment of CNTs can also be achieved by applying a magnetic field $[40,41]$ to singlewalled nanotube suspensions. In these reports, magnetic forces exerted a torque on objects placed in the field, and were used to entrap carbon nanotubes from suspensions and thereby to fabricate them into devices.

Magnetic field-induced alignment can also be achieved on polymeric chains. In another report, after a strong magnetic field was applied on CNTs which were dispersed in a polymeric matrix [18], polymer-mediated alignment of CNTs was achieved. In this polymer/CNTs system, the alignment was established from two sources: CNTs inside the polymeric matrix were reoriented by the magnetic field and the rheological force 
generated by polymer chains which were also susceptible to the magnetic field and had been magnetically aligned.

\subsubsection{Self assembly}

Self-assembly chemistry has been widely used to assemble nanomaterials into ordered structures and to tailor nanomaterials on the surface of metals or semiconductors [41] to achieve physical and chemical properties of an interface. This technique [36] has been used to align pre-grown SWNTs in a big scale by utilizing their high polarisability that enables CNTs to possess the affinity to polar groups. In this method, two distinct surface groups, polar group, such as amino $\left(-\mathrm{NH}_{2} /-\mathrm{NH}_{3}{ }^{+}\right)$or carboxylic acid $(-\mathrm{COOH})$ and nonpolar group, such as alkyl, were coated on different areas of a substrate. While being dipping the substrate into a SWNT suspension, SWNTs were attracted to the polar groups and self-assemble to form a templated structure with polar groups.

Besides rheological forces and magnetic forces, electric fields have been applied to align, manipulate, purify and select CNTs in suspensions. Electrophoresis and dielectrophoresis are terms to describe the motion of charged and neutral objects inside electric fields. The detailed discussion on application of electrophoresis and dielectrophoresis in postsynthesis treatment on carbon nanotubes is to be given in Chapter 5 .

\section{Reference:}

1. P. M. Ajayan, T. W. Ebbesen, T. Ichihashi, S. Iijima, K. Tanigaki, and H. Hiura (1993), Nature, 362: p. 522-525. 
2. K. D. Ausman, R. Piner, O. Lourie, and R. S. Ruff (2000), The journal of Physical Chemistry B, 104: p. 8911-8915.

3. J. L. Bahr, E. T. Mickelson, M. J. Bronikowski, R. E. Smalley, and J. M. Tour (2000), Chemical Communication: p. 193-194.

4. P. Ball (2003), Nature, 424: p. 143.

5. S. Banerjee and S. S. Wong (2002), Journal of American Chemistry Society, 124: p. $8940-8948$.

6. R. H. Baughman, A. A. Zakhidov, and W. A. D. Heer (2002), Science, 297: p. 787-792.

7. A. Bianco and M. Prato (2003), Advanced Materials, 15: p. 1765-1768.

8. J. M. Bonard, T. Stora, J.-P. Salvetat, F. Marier, T. Stockli, C. Duschl, L. Forro, W. A. d. Heer, and A. Chatelain (1997), Advanced Materials, 9: p. 827-831.

9. D. Charttopadhyay, I. Galeska, and F. Paradimitrakopoulos (2003), Journal of the American Chemical Society, 125: p. 3370-3375.

10. O. Chauvet, L. Forro, W. Bacsa, D. Ugarte, B. Doudin, and W. A. d. Heer (1995), Physics Review B, 52: p. R6963-R6966.

11. J. Chen, M. A. Hamon, H. Hu, Y. Chen, A. M. Rao, P. C. Eklund, and R. C. Haddon (1998), Science, 282: p. 95.

12. X. Q. Chen, T. Saito, H. Yamada, and K. Matsushige (2001), Applied Physics Letters, 78: p. 3714-3716.

13. Y. K. Chen, M. L. H. Green, J. L. Griffin, J. Hammer, R. M. Lago, and S. K. Tsang (1996), Advanced Materials, 8: p. 1012-1015.

14. A. C. Dillon, T. Gennett, K. M. Jones, J. L. Alleman, P. A. Parilla, and M. J. Heben (1999), Advanced Materials, 11: p. 1354-1358.

15. C. A. Dyke, M. P. Stewart, F. Maya, and J. M. Tour (2003), Synlett, 1: p. 1-6.

16. T. W. Ebbesen, P. M. Ajayan, H. Hiura, and K. Tanigaki (1994), Nature, 367: p. 519.

17. B. Gao, Guozhen, Z.Yue, Q. Qiu, Y. Cheng, H. Shimoda, L. Fleming, and O. Zhou (2001), Advanced Materials, 13: p. 1770-1773. 
18. H. Garmenstani, M. S. Al-Haik, K. Dahmen, R. Tannenbaun, D. Li, S. S. Sablin, and M. Y. Hussaini (2003), Advanced Materials, 15: p. 1998-1921.

19. W. A. d. Heer, W. S. Bacsa, A. Chatalain, T. Gerfin, R. Humphrey-Baker, L. Forro, and D. Ugarte (1995), Science, 268: p. 845-847.

20. H. Hiura, T. W. Ebbesen, and T. Tanigaki (1995), Advanced Materials, 7: p. 275276.

21. J. Hone, M. C. Llaguno, N. M. Nemes, A. T. Johnson, J. E. Fischer, D. A. Walters, M. J. Casavant, J. Schmidt, and R. E. Smalley (2000), Applied Physics Letters, 77: p. 666-668.

22. K. Kamaras, M. E. Itkis, H. Hu, B. Zhao, and R. C. Haddon (2003), Science, 301: p. 1501.

23. K. Keren, R. S. Berman, E. Buchstab, U. Srivan, and E. Braun (2003), Science, 302: p. 1380-138.

24. H. J. Kim, K. K. Jeon, K. H. An, C. Kim, J. G. Heo, S. C. Lim, D. J. Bae, and Y. H. Lee (2003), Advanced Materials, 20: p. 1757-1760.

25. J. Kong, N. R. Franklin, C. Zhou, M. G. Chapline, S. Peng, K. Cho, and H. Dai (2000), Science, 287: p. 622-625.

26. R. Krupke, F. Hennrich, H. v. Loneysen, and M. M. Kappes (2003), Science, 301: p. 344-347.

27. R. Krupke, F. Hennrich, H. B. Weber, M. M. Kappes, and H. v. Loneysen (2003), Nano Letters, 3: p. 1019-1023.

28. S. W. Lee, D. S. Lee, H. Y. Yu, E. E. B. Campbell, and Y. W. Park (2004), Applied Physics A, 78: p. 283-286.

29. L.-J. Li, R. J. Nicholas, C.-Y. Chen, R. C. Darton, and S. C. Baker (2005), Nanotechnology, 16: p. S202-S205.

30. K. Y. Lim, C. H. Sow, J. Lin, F. C. Cheong, Z. X. Shen, J. T. L. THong, K. C. Chin, and A. T. S. wee (2003), Advanced Materials, 15: p. 300-303.

31. Y. Lin, B. Zhou, K. A. S. Fernando, P. Liu, L. F. Allard, and Y.-P. Sun (2003), Macromolecules, 36: p. 7199-7204.

32. J. Liu, A. G. Rinzler, H. Dai, J. H. Hafner, R. K. Bradley, P. J. Boul, A. Lu, T. Iverson, K. Shelimov, C. B. Huffman, F. Rodriguez-Macias, Y.-S. Shon, T. R. Lee, D. T. Colbert, and R. E. Smalley (1998), Science, 280: p. 1253-1256. 
33. L. A. Nagahara, I. Amlani, J. Lewenstein, and R. K. Tsui (2002), Applied Physics Letters, 80: p. 3826-3828.

34. R. M. Pashley and M. E. Karaman (2004), in book. Applied Colloid and Surface Chemistry West Sussex: John Wiley \& Sons Ltd

35. M. S. Raghuveer, P. G. Ganesan, J. D'Arcy-Gall, and G. Ramanath (2004), Applied Physics Letters, 84: p. 4484-4486.

36. S. G. Rao, L. Huang, W. Setyawan, and S. Hong (2003), Nature, 425: p. 36-37.

37. M. S. P. Shaffer, X. Fan, and A. H. Windle (1998), Carbon, 36: p. 1603-1612.

38. K. B. Shelimov, R. O. Esenaliev, A. G. Rinzler, C. B. Huffman, and R. E. Smalley (1998), Chemical Physics Letters, 282: p. 429-434.

39. J. M. Skowronski, P. Scharff, N. Pfander, and S. Cui (2003), Advanced Materials, 15: p. 55-57.

40. B. W. Smith, Z. Benes, D. E. Luzzi, and J. E. Fischer (2000), Applied Physics Letters, 77: p. 663-665.

41. R. K. Smith, P. A. Lewis, and P. S. Weiss (2004), Progress in Surface Science, 75 : p. 1-68.

42. M. S. Strano, http://www. scs.uinc.edu/chem eng/Faculty/strano.htm.

43. M. S. Strano, C. A. Dyke, M. L. Usrey, P. W. Barone, M. J. Allen, H. Shan, C. Kittrell, R. H. Hauge, J. M. Tour, and R. E. Smalley (2003), Science, 301: p. 1519-1521.

44. J. Suehiro, G. Zhou, and M. Hara (2003), Journal of Physcis D: Applied Physics, 36: p. L109-L114.

45. Y.-P. Sun, W. Huang, Y. Lin, K. Fu, A. Kitaygorodskiy, L. A. Riddle, Y. J. Yu, and D. L. Carroll (2001), Chemical Materials, 13: p. 2864-2869.

46. K. Tohji, T. Goto, H. Takahashi, Y. Shinoda, N. Shimizu, B. Jeyadevan, I. Matsuoka, Y. Saito, A. Kasuya, T. Ohsuna, K. Hiraga, and Y. Nishina (1996), Nature, 383: p. 679.

47. S. C. Tsang, P. J. F. Harris, and M. L. H. Green (1993), Nature, 362: p. 362-522.

48. S. S. Wong, E. Joselevich, A. T. Woolley, C. L. Cheung, and C. M. Lieber (1998), Nature, 394: p. 52-54. 
49. S. S. Wong, A. T. Woolley, E. Joselevich, C. L. Cheung, and C. M. Lieber (1998), Journal of Physics D: Applied Physics, 120: p. 8557-8558.

50. K. Yamamoto, S. Akita, and Y. Nakayama (1998), Journal of Physcis D: Applied Physics, 31: p. L34-L36.

51. P. Yang (2003), in book. The Chemistry of Nanostructured Materials Singapore: World Scientific

52. M. Zheng, A. Jagota, E. D. Semke, B. A. Diner, R. S. Mclean, S. R. Lustig, R. E. Richardson, and N. G. Tassi (2003), Nature Materials, 2: p. 338-342.

53. M. Zheng, A. Jagota, M. S. Strano, A. P. Santos, P. Barone, S. G. Chou, B. A. Diner, M. S. Dresselhaus, R. S. Mclean, G. B. Onoa, G. Samsonidze, E. D. Semke, M. Usrey, and D. J. Walls (2003), Science, 302: p. 1545-1548. 


\section{Chapter 5}

\section{Dielectrophoresis}

\subsection{Definition}

The word dielectrophoresis originates from the Greek word phoresis, which means carrying. Dielectrophoresis [11] is termed as the translational motion of a neutral matter caused by its response to an inhomogeneous electric field. The force generated by the electric field to produce this response is called the dielectrophoretic force. The translational response can be coupled to a moment arm, which produces torsion on the object in the non-uniform electric field, and results in an alignment of axially asymmetric object in the field.

Dielectrophoresis should be distinguished from another phenomenon of matter motion, electrophoresis [10, 12]. As shown in Figure 5-1, dielectrophoretic (DEP) force moves a neutral body toward the strongest field region while electrophoresis refers to the motion of a charged body in an electric field.

In the case of an anisotropic object in a non-uniform electric field, a picture can be drawn that the electric field produces a torque, then a translational motion is generated on an object inside the field and the particle is to be pulled toward either electrode. If the particle is isotropic or spherically symmetric, torque will not arise. 


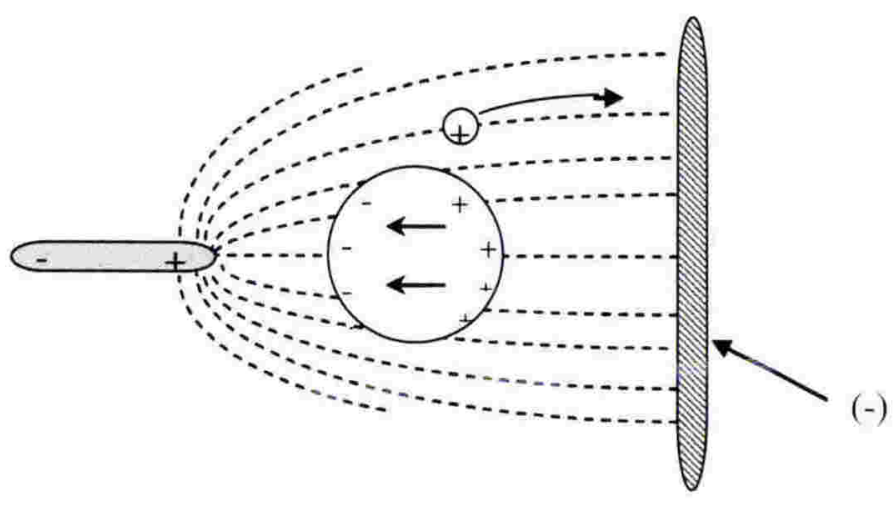

Figure 5-1 Behaviours of neutral and charged bodies in a non-uniform electric field, with dashed lines indicating its direction. The big sphere in the diagram represents a neutral body and the small sphere represents a charged body. The neutral body is polarised and pulled toward the strongest field region. The charged body moves along the electric field lines. The parallel arrows inside the neutral body and the bend arrow outside the charged body indicate their movements inside the electric field.

In a non-uniform electric field, as shown in Figure 5-1, different behaviours are exhibited by charged and neutral particles. The charged particle is pulled to the electrode with opposite polarity whilst a force orthogonal to the field direction is exerted upon the neutral particle. In a neutral object, the numbers of the positive and the negative charges are equal to each other. Orthogonal translational motion occurs in the domain of a nonuniform electric field due to polarisation of the neutral body and the negative charges and the positive charges of the body are pulled to the sides closest to the electrodes with opposite polarities. When the electric field exerts on two regions inside the particle, the strength of the local fields are not equal. The polarisation of a neutral body in a nonuniform field brings about a torque pulling the body towards the region of stronger gradient field.

\subsection{Polarisation}

Polarisation inside a molecule consists of four modes: 
(1) Electronic polarisation, which arises from the distortion of the electron orbitals under the impact of the external field. However, the contribution from electronic polarisation is very small because the intensity of the external field is much less than that of the internal field inside the atoms;

(2) Atomic polarisation, which comes from the shifts of charged atoms with respects to each other inside a molecule. In organic compounds, this contribution to the total polarisation is usually small whereas, in inorganic compounds, and it can be very large because of the density of charges;

(3) Orientational or dipolar polarisation, which arises from the orientational responses of molecules or parts of molecules that possess a permanent dipole moment. They will respond to the external electric field by aligning with it to reduce their potential. This type of polarisation can be quite large;

(4) Nomadic charge polarisation, which results from the pliant responses of thermally excited charges situated on long domains. This occurs where there are long chains or lattices of molecules with dislocated charges.

The long electron mean path and the geometric structure of carbon nanotubes, can be expected to result in a large nomadic polarisation effect.

\subsection{Dielectrophoretic force and Torque}

When a small neutral body is placed in a static electric field at equilibrium, the net electric force $\boldsymbol{F}[11]$ upon it is

$$
\boldsymbol{F}=(\boldsymbol{P} \cdot \nabla) \boldsymbol{E}_{e}
$$


where $\boldsymbol{P}$ is the polarisation, and $\boldsymbol{E}_{\boldsymbol{e}}$ is the external electric field. Therefore, $\nabla \boldsymbol{E}_{\boldsymbol{e}}$ is the field gradient.

If the dielectric body is isotropically, linearly, and homogeneously polarisable, then

$$
\boldsymbol{P}=\boldsymbol{\alpha}_{\mathrm{v}} \boldsymbol{E}_{\boldsymbol{e}}
$$

where $\boldsymbol{\alpha}$ is the tensor polarisation per unit volume in unit field, $v$ is the volume of the body, and $\boldsymbol{E}_{\boldsymbol{e}}$ is the external field, then the net electric force for an ideal linear neutral body inside an electric field can be rewritten as

$$
\begin{aligned}
\boldsymbol{F} & =\boldsymbol{\alpha}_{\mathrm{v}}\left(\boldsymbol{E}_{\boldsymbol{e}} \cdot \nabla\right) \boldsymbol{E}_{\boldsymbol{e}} \\
& =\frac{\boldsymbol{\alpha}}{2} \vee \nabla\left|\boldsymbol{E}_{e}\right|^{2}=\boldsymbol{\alpha}_{\mathrm{v}}\left|\boldsymbol{E}_{\boldsymbol{e}}\right| \nabla\left|\boldsymbol{E}_{\boldsymbol{e}}\right|
\end{aligned}
$$

For a sphere, $v=4 \pi \mathrm{a}^{3} / 3$, where $\mathrm{a}$ is the radius of the sphere in an infinite medium of permittivity $\varepsilon_{1}$, and in a uniform external electric field strength, $\boldsymbol{P}_{\text {is given as }}$

$$
\begin{aligned}
& \boldsymbol{P}=\boldsymbol{\alpha}_{\mathrm{V}} \boldsymbol{E}_{\mathrm{e}}=4 \pi a^{3} \varepsilon_{1}\left(\frac{\varepsilon_{2}-\varepsilon_{1}}{\varepsilon_{2}+2 \varepsilon_{1}}\right) \boldsymbol{E}_{e} \\
& \boldsymbol{\alpha}_{\mathrm{v}}=4 \pi a^{3} \varepsilon_{1}\left(\frac{\varepsilon_{2}-\varepsilon_{1}}{\varepsilon_{2}+2 \varepsilon_{1}}\right)=4 \pi a^{3} \varepsilon_{0} K_{1}\left(\frac{K_{2}-K_{1}}{K_{2}+2 K_{1}}\right)
\end{aligned}
$$

hence

$$
\boldsymbol{F}=2 \pi a^{3} K_{1} \varepsilon_{0}\left(\frac{K_{2}-K_{1}}{K_{2}+2 K_{1}}\right) \nabla\left|\boldsymbol{E}_{\boldsymbol{e}}\right|^{2}
$$

where the term $\left(\mathrm{K}_{2}-\mathrm{K}_{1}\right)$ is introduced due to the replacement of the dielectric of the medium by that of the sphere, $\varepsilon_{l}=\varepsilon_{0} \mathrm{~K}_{1}$ and $\varepsilon_{2}=\varepsilon_{0} \mathrm{~K}_{2}$, are the absolute permittivities of media 1 and 2 respectively. Here $\varepsilon_{0}$ is the permittivity of free space, a constant with an approximate value of $8.854 \times 10^{-12} \mathrm{C} \mathrm{V}^{-1} \mathrm{~m}^{-1}$. Therefore, the net electric force can be rewritten as 
Chapter 5 Dielectrophoresis

$$
\boldsymbol{F}=\frac{3}{2} \mathrm{v} \varepsilon_{1} \frac{\varepsilon_{2}-\varepsilon_{1}}{\varepsilon_{2}+2 \varepsilon_{1}} \nabla\left|\boldsymbol{E}_{\boldsymbol{e}}\right|^{2}
$$

It should be emphasized here that the force is dependent on the square of the gradient field regardless of the direction of the field. To maximize the force, both large fields $\left|\boldsymbol{E}_{\boldsymbol{e}}\right|$ and large field gradients, $\nabla\left|\boldsymbol{E}_{\boldsymbol{e}}\right|$, should be applied. Experimentally, $\nabla\left|\boldsymbol{E}_{\boldsymbol{e}}\right|$, can be increased by using electrodes with small radii of curvature.

Studies of dielectrophoretic response of particles, which are suspended in liquid media, show that the response is also strongly dependent on the frequency of the electric field, the conductivity and the permeability of the particle and the medium.

\subsection{Dielectrophoresis and carbon nanotubes}

\subsubsection{Theoretical background}

The idea of using dielectrophoresis technique for aligning and manipulating carbon nanotubes was first theoretically proposed by B. H. Fishbine [3] in 1996. A CNT can be regarded as a linear, rigid and conducting cylinder with a very large length/diameter ratio. Accordingly, it should behave as a one-dimensional conductor with high polarisability along one axis. These facts with CNTs make them experience high dielectrophoretic forces $[4,11]$ when they are placed in a non-uniform electric field.

The energy of interaction between a cylinder, such as a CNT, and an applied electric field can be modelled in the following way:

In the high field limit, the polarisation of the nanotube is given by

$$
\boldsymbol{P}=-\alpha_{\mathrm{CNT}}\left|\boldsymbol{E}_{e}\right| \cos \theta
$$


where $\alpha_{\mathrm{CNT}}$ represents the axial polarisability of the CNT, and $\theta(-\pi / 2<\theta<\pi / 2)$ defines the angle of nanotube axis to the applied field, as seen in Figure 5-2.

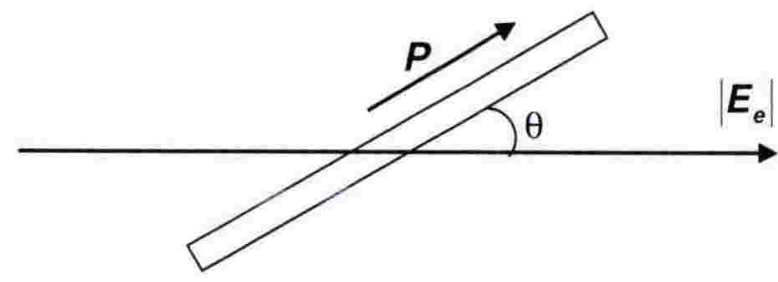

Figure 5-2 Polarisation of a cylindrical conductor in an electric field.

Therefore, energy of the interaction

$$
\boldsymbol{U}=-\frac{1}{2} \boldsymbol{P} \boldsymbol{E}_{\boldsymbol{e}}=\frac{1}{2} \alpha_{\mathrm{CNT}} \cos ^{2} \theta\left|\boldsymbol{E}_{\boldsymbol{e}}\right|^{2}
$$

Force on the nanotube

$$
\boldsymbol{F}=\nabla|\boldsymbol{U}|=\frac{1}{2} \alpha_{\mathrm{CNT}} \nabla\left|\boldsymbol{E}_{e}\right|^{2} \cos ^{2} \theta
$$

And torque on the nanotube

$$
\Gamma=\frac{\partial \boldsymbol{U}}{\partial \theta}=-\frac{1}{2} \alpha_{\mathrm{CNT}}\left|\boldsymbol{E}_{e}\right|^{2} \sin 2 \theta
$$

From the equations (5-10) and (5-11) and the plots of DEP force and DEP torque versus the angle of the CNT to the electric field in Figure 5-3, one can tell that

(1) As demonstrated in Figure 5-3 (a), the magnitude of the DEP force on the CNT is at maximum when the CNT is parallel to the electric field and it is at minimum when perpendicular to the field.

(2) As demonstrated in Figure 5-3 (b), the absolute magnitude of the DEP torque on the CNT is at minimum when the CNT is either parallel or perpendicular to the electric field, and is at maximum when $\theta$ is $\pm \pi / 4$. 

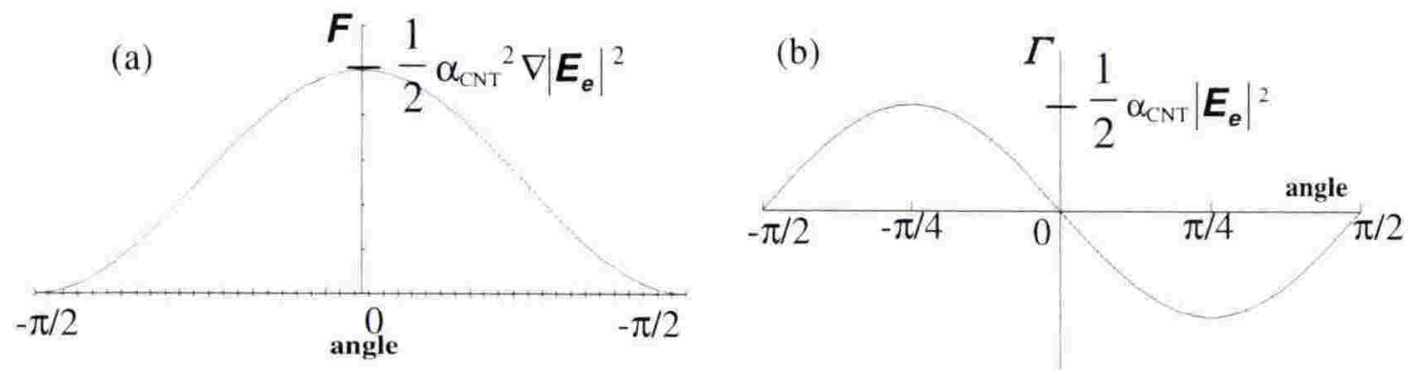

Figure 5-3 Plots of (a) DEP force and (b) DEP torque on a CNT versus the angle of CNT to the electric field.

It can be drawn from Figure 5-3 that CNTs experience the maximum DEP force and a zero torque when they are aligned across the electrodes $(\theta=0)$ in a non-uniform field. Therefore the aligning of CNTs along the direction of the field is the most stable state for CNTs when the electric field was applied.

\subsubsection{Applications of dielectrophoresis for post-synthesis treatment of CNTs}

According to Fishbine, alignment of CNTs by an electric method has the following advantages:

(1) This method has no restrictions on material thickness. Unlike the aligned CNT forests grown by CVD, the dielectrophoretic alignment from suspensions can control the length of alignment, just by adjusting the distance between opposite electrodes; therefore it can be particularly useful, for example, when establishing CNT alignment through a volume of polymer composite.

(2) The direction of the alignment can be modified by changing the layout of the electrodes. In the case of CVD growing, CNTs can only orientate in a direction 
perpendicular to the growth substrate. Dielectrophoretic alignment from solutions can be at any angle on the plane of the substrate.

(3) Density, dimensions, conductivity and helicity of aligned CNTs by electric methods can be controlled, by changing the parameters of the experimental conditions.

Dielectrophoresis techniques have been applied to carbon nanotubes for post-synthesis treatment purposes: alignment, purification, selection and manipulation. A sufficiently high field will not only align long and polarisable tubes, but also can be used to separate conducting CNTs from non-conducting ones and less-conductive impurities, as well as to manipulate CNTs when fabricating CNT-based devices.

\section{Aligning CNTs}

The earliest experimental attempt of aligning CNTs by an electric method can be traced back to 1996 when Yamamoto et al. [12] applied DC and AC electric fields onto multiwalled carbon nanotubes suspensions. In this report, alignment of CNTs between electrodes was obtained and attributed to the anisotropy of their electrophoresis velocity, which depends on the shape of the particles. In Yamamoto's following work, AC electric fields with frequencies from $10 \mathrm{~Hz}$ to $10 \mathrm{MHz}$ were applied to MWNTs suspensions. The degree of the orientation of CNTs along the electric field was observed to increase with increasing frequency of the electric field. However, a feasible mechanism has not been given on this observation in Yamamoto's report.

Following the success of aligning MWNTs by AC electric field, a later report, which was reported by Chen et al. [2], demonstrated that applying this technique to SWNTs is also viable. This was explained in the light of the electronic polarisation induced in carbon nanotubes by the electric field. The dipole moment in the direction parallel to the tube 
axis is much stronger than the perpendicular direction because of the anisotropic structure of CNTs. Also, they observed that, by increasing the frequency and magnitude of the electric field, the alignment of SWNTs was improved. However, a rational explanation for this frequency-dependency was not available in their report.

As what we discussed from the previous section, in a non-uniform electric field, CNTs experience the maximum DEP force and a zero torque when they are aligned across the electrodes. Mechanically, the most favourable position for CNTs is to follow the gradient electric field. Furthermore, a high frequency of AC electric field favours the alignment. These observations and models naturally imply that it should be possible to trap and purify CNTs by high-frequency AC dielectrophoresis, based on the fact that objects with different geometric shapes behave differently in an alternating electric field.

\section{Placement of CNTs}

The alignment of CNTs by electric field has been extended to trapping suspended CNTs into nanometre-scale assemblies. Reports from different sources [6-9] have been made on placement of suspended CNTs by AC electric fields. In these reports, dielectrophoresis was combined with a lithographic method to integrate CNT-based devices in a dimension of 200 to $300 \mathrm{~nm}$. It should be noted here that trapping with this method selectively favoured metallic SWNTs.

It was suggested by Park et al. [8] that this method has some advantages: "(1) Simultaneous response of CNTs in suspensions to electric field was observed: only a short alignment time of 1 2 seconds is required, (2) the position and direction of the suspended CNTs can be achieved and controlled accurately." 


\section{Separation of CNTs}

As reviewed in Chapter 4, separation of metallic single-walled CNTs from semiconducting ones remains one of major hurdles against large-scale applications of CNTs because the synthesis methods have little tendency towards the selective formation of either type of CNTs till now. Post-synthesis selection work on CNTs is required for further development of certain applications.

As reported by Krupke et al. [5], when an electric field was applied to CNT suspensions, metallic tubes were deposited on electrodes and semiconducting ones, however, remained in suspension because of the differences in their dielectrophoresis behaviour. An enrichment of up to $80 \%$ of metallic CNTs has been observed on the electrodes after a high-frequency DEP process.

This phenomenon can be understood by using equation (5-10)

$$
\boldsymbol{F}=\nabla \boldsymbol{U} \mid=\left(\alpha_{\mathrm{CNT}} \nabla\left|\boldsymbol{E}_{\mathrm{e}}\right|^{2} \cos ^{2} \theta\right) / 2,
$$

Where $\alpha_{C N T}$ is proportional to the number of free nomadic electrons, n. For a semiconducting SWNT of identical dimensions to a metallic SWNT, the number of free electrons can be expected to be approximated as

$$
\mathrm{n}_{\text {semi }} \sim \mathrm{n}_{\text {metallic }} \mathrm{e}^{-\mathrm{Eg} / \mathrm{k} \mathrm{T}}
$$

where $\mathrm{n}_{\text {metallic }}$ is the number of free electrons of metallic CNT, $\mathrm{E}_{\mathrm{g}}$ is the bandgap of semiconducting CNT, $\mathrm{k}_{\mathrm{B}}$ is Boltzman constant and $\mathrm{T}$ is the temperature. For $\mathrm{E}_{\mathrm{g}} \sim 1 \mathrm{eV}$, a

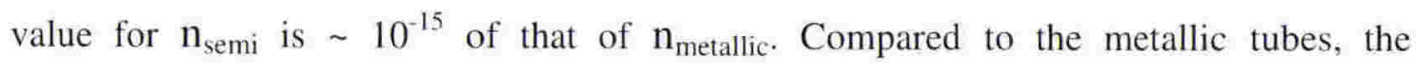
magnitude of the DEP force which semiconducting tubes experience in the electric field is negligibly small. 
The DEP force exerted for a homogeneous dielectric object, can be defined by using the equation (5-7)

$$
\boldsymbol{F} \propto \boldsymbol{\varepsilon}_{1} \frac{\boldsymbol{\varepsilon}_{2}-\boldsymbol{\varepsilon}_{1}}{\varepsilon_{2}+2 \varepsilon_{1}} \nabla\left|\boldsymbol{E}_{\boldsymbol{e}}\right|^{2}
$$

where $\varepsilon_{1}$ is the dielectric constant of the aqueous media and $\varepsilon_{2}$ is the dielectric constant of the object experiencing DEP. It can be seen that if $\varepsilon_{1}>\varepsilon_{2}$, the object can be expected to experience negative DEP (be repelled from areas of high field strength) whilst if $\varepsilon_{2}$ $>\mathcal{E}_{1}$, the object experiences positive DEP (be attracted from regions of high field strength). Under the same electric field, if we ignore the volume difference between CNTs, every parameter but the dielectric constant of the CNTs remains the same. Taking the distilled water as the medium, the dielectric constant $\varepsilon_{1}$ is 80 .

The dielectric constant of semiconducting CNTs [1] has been given as

$$
\varepsilon_{s} \cong 1+\left(\frac{\hbar \omega_{p}}{5.4 E_{g}}\right)^{2}
$$

where $\hbar \omega_{p}$ is the energy of the plasma oscillation along the CNT axis with a value of approximately $5 \mathrm{eV}$ and $E_{g}$ is the band gap of the semiconducting CNTs. Calculations of $\varepsilon_{s}$ (replacing $\varepsilon_{2}$ in equation (5-7) for semiconducting tubes) give that $\varepsilon_{s}$ is less than 5 . On the other hand, metallic CNTs have large number of dielectric electrons and as a result, their dielectric constant is a huge number.

Therefore, the DEP force exerted on metallic CNTs is positive while that on semiconducting CNTs is negative. This difference results in the collection of metallic CNTs on electrodes while semiconducting CNTs were repelled from the electrodes and remained in the suspension. 
Chapter 5 Dielectrophoresis

In the experimental work to be introduced from the next chapter, an ac electric field at radio frequencies was used to attract and align the tubes. In chapter 7 , a prototype device was fabricated with the aim of separating MWNTs from as-grown samples, based on the dielectrophoresis theory.

\section{Reference:}

1. L. X. Benedict, S. G. Louie, and M. L. Cohen, Physical Review B, 1995. 52: p. 8541-8549.

2. X. Q. Chen, T. Saito, H. Yamada, and K. Matsushige, Applied Physics Letters, 2001. 78: p. 3714-3716.

3. B. H. Fishbine, Fullerenes Science \& Technology, 1996. 41: p. 87-100.

4. T. B. Jones, in book. Electromechanics of Particles. 1995, Cambridge: Cambridge University Press

5. R. Krupke, F. Hennrich, H. v. Loneysen, and M. M. Kappes, Science, 2003. 301: p. 344-347.

6. R. Krupke, F. Hennrich, H. B. Weber, D. Beckmann, O. Hampe, S. Malik, M. M. Kappes, and H. V. Lonneysen, Applied Physics A, 2003. 76: p. 397-400.

7. R. Krupke, F. Hennrich, H. B. Weber, M. M. Kappes, and H. V. Loneysen, Nano Letters, 2003. 3: p. 1019-1023.

8. S. W. Lee, D. S. Lee, H. Y. Yu, E. E. B. Campbell, and Y. W. Park, Applied Physics A, 2004. 78: p. 283-286.

9. L. A. Nagahara, I. Amlani, J. Lewenstein, and R. K. Tsui, Applied Physics Letters, 2002. 80: p. 3826-3828.

10. H. Nishijima, Applied Physics, 1999. 74: p. 4061-4063. 
Chapter 5 Dielectrophoresis

11. H. A. Pohl, in book. Dielectrophoresis: The Behavior of Neutral Matter in Nonuniform Electric Fields 1st ed. 1978, Cambridge: Cambridge University Press

12. K. Yamamoto, S. Akita, and Y. Nakayama, Japan Journal of Applied Physics, 1996. 35: p. 917-918. 


\section{Chapter 6}

\section{Alignment of Carbon Nanotubes by Dielectrophoresis}

The introduction to the theory of dielectrophoresis in the previous chapter illustrated that an object with a one-dimensional structure such as a carbon nanotube has a highly anisotopic polarisability and experiences both dielectrophoretic force and a dielectrophoretic torque in a non-uniform AC electric field. This phenomenon can be utilized in the post-synthesis treatment and manipulation of carbon nanotubes.

Applying well-dispersed carbon nanotubes suspensions in a non-uniform electric field at a high frequency will generate a dielectrophoretic force upon carbon nanotubes, and as a result, this force will align carbon nanotubes along the electric field vector. In this chapter, descriptions are given on dielectrophoresis techniques used for the purpose of aligning multi-walled carbon nanotubes from two different precursors, and the related results are presented.

\subsection{Experimental}

\subsubsection{Materials and Methods}

\section{Nanotubes}

Multi-walled carbon nanotubes (MWNT) were grown on a silica plate from iron cluster catalysts derived by pyrolysis of iron (II) phthalocyanine and of ferrocene, as described in Chapter 3. MWNTs were peeled off from the plate by a razor blade. 


\section{Nanotube Suspension}

\section{a. Surfactants and solvents}

An ionic surfactant, sodium dodecyl sulphate (abbreviated as SDS hereunder, melting point: $204-207{ }^{\circ} \mathrm{C}$, solid in room temperature) and a non-ionic surfactant, Triton X-100 (melting point: $6{ }^{\circ} \mathrm{C}$, liquid in room temperature), were considered in the experiments. However, Triton $\mathrm{X}-100$ was chosen as the surfactant for the alignment of MWNTs because according to our own observation in experiments, SDS is more polar than Triton $\mathrm{X}-100$ and undergoes crystallization into particles after the solvent is evaporated, which retards the movement of CNTs and inhibit further characterisation.

Three polar solvents were also used to suspend MWNT samples. Comparison was made among the impacts of the solvents on the changes of the capacitance components and $\mathrm{AC}$ conductance on simultaneous dielectric spectroscopy.

\section{b. Preparation of suspension}

As-grown MWNTs were suspended by mixing them in the ratio of $1.0 \mathrm{mg}$ (MWNT): $1 \mathrm{~mL}$ (absolute ethanol, isopropanol or distilled water): $15 \mathrm{~mL}$ (1\% Triton X-100 in distilled water, a non-ionic surfactant). After 30 minutes in an ultrasonic bath with a power of $40 \mathrm{w}, 15 \mu \mathrm{L}$ of the suspension were re-mixed with $1 \mathrm{~mL}$ absolute ethanol, isopropanol or distilled water and a further $15 \mathrm{~mL} 1 \%$ Triton X-100. A further 15 minutes ultrasonication gave the working suspension with a concentration of $15 \mu \mathrm{g}$ CNTs per ml.

\subsubsection{Apparatus}

Interdigitated Electrodes (abbreviated as IDEs hereunder, fabricated by Rob Kemp, Industrial Research Limited) upon glass substrates were used in the experiment. These 
structures were laid out as interpenetrating arrays of fingers, which connected to two different larger arms, through which an external power from a signal generator was supplied. There are same numbers of parallel fingers of opposite polarity. The microstructure of an inter-digitated electrode array (IDE) used in the experiment has been described in details in references [1-3]. The IDE arrays were patterned onto square glass substrates $(5 \mathrm{~mm} \times 5 \mathrm{~mm})$, as shown in Figure 6-1. Three individually different layouts of IDEs were employed in the experiment.

(1) IDE 1: A castle-wall pattern with regular dents of half circles (with diameter of 25 $\mu \mathrm{m}$ each) inside the $100 \mu \mathrm{m}$ wide electrode fingers (see 6-1 (a)). The closest distances between the castle-wall edges are all $35 \mu \mathrm{m}$.

(2) IDE 2: Another castle-wall pattern, with the castellation in squares with sides of $120 \mu \mathrm{m}$ (see 6-1 (b)) and $100 \mu \mathrm{m}$ apart. The closest distances between the castellation edges from the adjacent electrodes are all $150 \mu \mathrm{m}$.

(3) IDE 3: Parallel patterns (see 6-1 (c)), in which the electrode fingers are in linear forms and the electric field has no periodical geometrical effect. Each finger is 10 $\mu \mathrm{m}$ wide, with $40 \mu \mathrm{m}$ gaps between the electrodes.

All of the electrode fingers in the IDEs demonstrated above consist of $300 \mathrm{~nm}$ thick Au over a $\mathrm{Ti}$ layer with a thickness of $30 \mathrm{~nm}$, fabricated on glass substrates by a lithographical method. In all the IDE structures, opposite interdigitated electrodes in parallel were linked to two main electrode arms ( $200 \mu \mathrm{m}$ wide, $1.8 \mathrm{~mm}$ apart), to which AC voltage can be applied. A glass cylinder of a size depending on the size of the IDE, was glued on top of the electrodes, and was used as a reservoir for suspensions. 
Chapter 6 Alignment of Carbon Nanotubes by Dielectrophoresis

Real-time monitoring of the alignment process on an IDE chip was conducted by video microscopy using a Nikon inverted microscope with $20 \mathrm{X}$ or $40 \mathrm{X}$ objectives, a colour CCD camera and an SVHS video recorder.

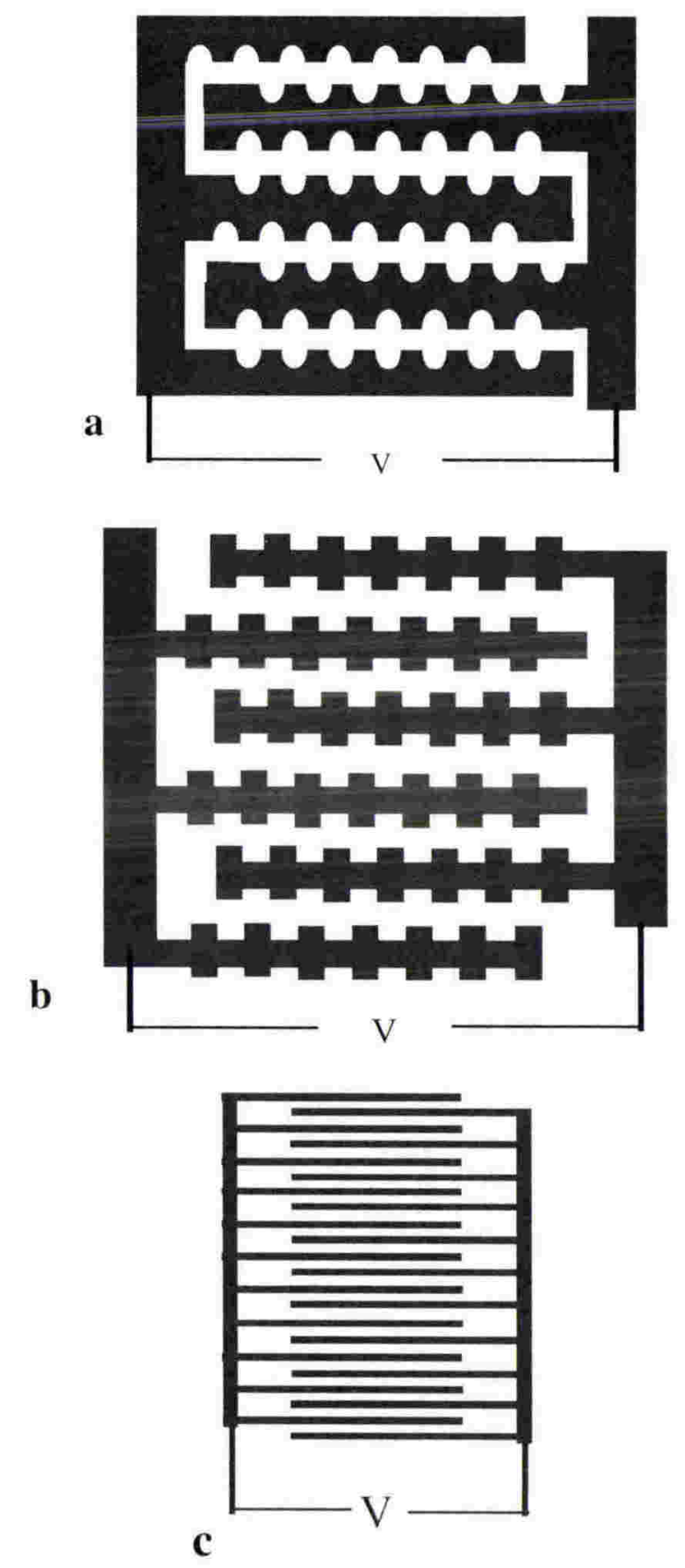

Figure 6-1 Diagrams of typical structures of IDEs. (a) IDE1, (b) IDE2, (c) IDE3. 


\subsubsection{Alignment Procedure}

A peak-to-peak sine-wave of $2.0 \mathrm{~V}$ at $4.15 \mathrm{MHz}$ was applied across an IDE 1, having feature size and electrode separation as described above. A sample $(150 \mu \mathrm{L})$ of the solvent (blank) or of the suspension was added onto the IDE chip and the power was kept on for $20-70$ minutes. During the collection and alignment, video-microscopy gave images on alignment processes.

A HP4194A impedance analyser tested the capacitance component and AC conductance (hereunder they are represented by capacitance and conductance) between the electrodes. After complete evaporation of the solvent, the CNT structures produced by dielectrophoresis were also investigated by SEM microscopy.

\subsubsection{Simultaneous Dielectric Spectroscopy}

An electrical bridge circuit that permits simultaneous dielectrophoresis and dielectric spectroscopy was used as described elsewhere [2]. The circuit diagram was depicted in Figure 6-2. The experimental set-up utilises a balanced "transmission-line" transformer wound onto a ferrite toroid to supply equal voltages to opposite sides of the bridge. When capacitance (an air variable capacitor) and resistance (a ten-turn cermet trimmer) are adjusted to the same value as the parallel impedance of the IDE, the HP4194A impedance analyser measures the parallel combination of the two arms of the bridge, plus the capacitance of the connecting co-axial cable. The suspension of CNTs was held in a cylindrical reservoir, which had been seamlessly glued on the electrode substrate. The impedance analyser monitored the real time changes of parallel due to the presence of the CNTs. The analyser also scanned the impedances versus frequency to determine whether 
the bridging between the opposite electrodes of IDEs is dominated by resistance change or capacitance change.

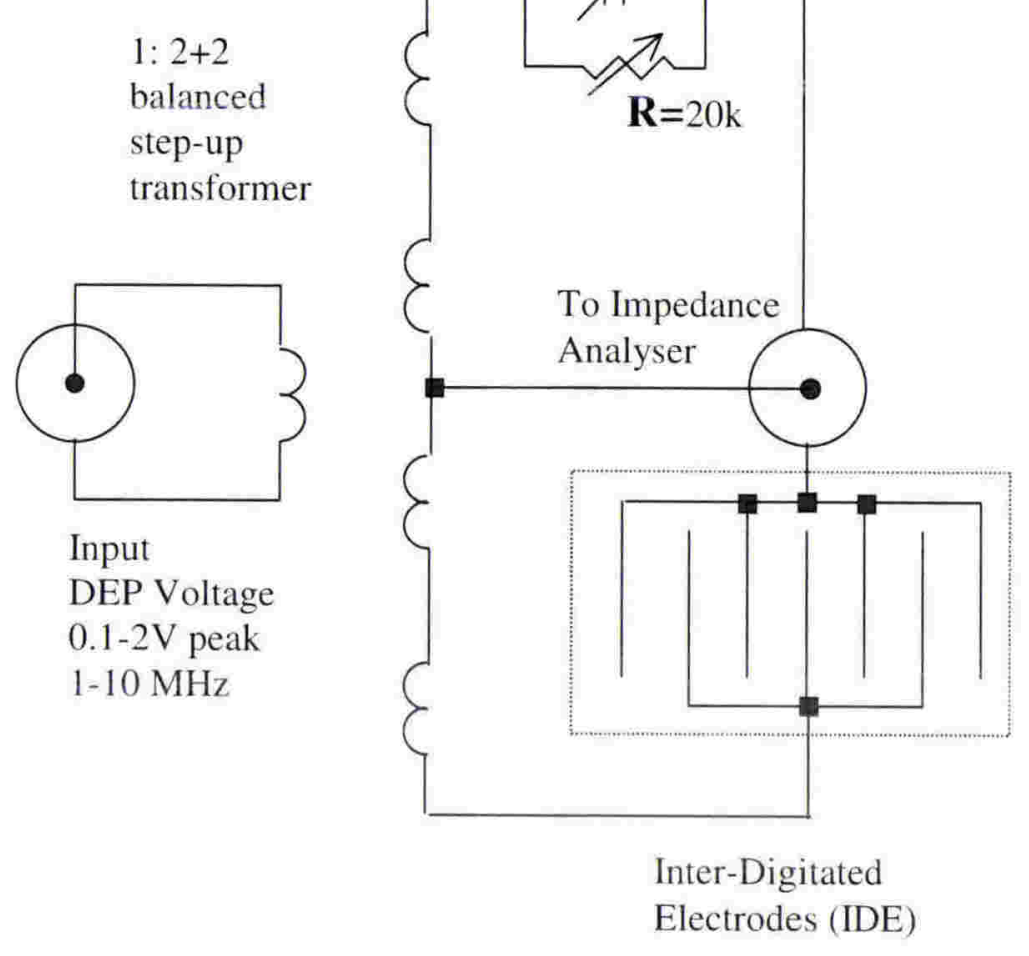

Figure 6-2 Bridge arrangement for real-time impedance monitoring of nanotube collections.

\subsubsection{Response of accumulated CNTs to on/off switching of the electric field}

A further experiment was performed to test the response of the CNT bridging to the exerted electric field. A $30 \mathrm{~V}$ peak radio-frequency voltage at $13.6 \mathrm{MHz}$ was applied for a certain time to an IDE 2 array with electrode gaps of $150 \mu \mathrm{m}$ within a suspension of CNTs in distilled water. The time responses of the conductance and capacitance were to follow as the $30 \mathrm{~V}$ signal was switched on and off. This is shown in Figure 6-7. During 
this procedure, an optical microscope, shown in Figure 6-8, observed the alignment of CNTs between the opposite electrodes. The CNT samples were made from FePC precursors.

\subsubsection{Frequency dependence investigation}

1. Dependence of dielectrophoresis on frequency

The dependence of dielectrophoresis on frequency was investigated by aligning CNTs on an IDE 1 at four discrete frequencies, $11 \mathrm{KHz}, 110 \mathrm{KHz}, 1.1 \mathrm{MHz}$ and $11 \mathrm{MHz}$, followed by observing them under SEM. IDE 1 was employed and the CNT samples were made from FePC precursors. The conditions of the investigation were kept the same as what was described in the alignment procedure in Section 6.1.3 except for the frequencies.

\section{Frequency scan}

Frequency scan was conducted on the sample from FePC precursors on an IDE 1, ranging from $10 \mathrm{KHz}$ to $400 \mathrm{KHz}$, after the alignment of CNTs had been achieved between electrodes and the solvent had been evaporated.

\subsubsection{A 4-electrode structure}

\section{The structure}

A 4-pad structure patterned on a glass substrate $(5 \mathrm{~mm} \times 5 \mathrm{~mm})$ was employed here. As shown in Figure 6-3, the structure consists of four $\mathrm{Al}$ pads, $4 \mathrm{~mm}$ each apart from the neighbouring pads and four $3 \mu \mathrm{m}$ wide $\mathrm{Al}$ electrodes, two of which are parallel to each other while the other two are in a pin-pin structure. A voltage can be applied through any 
two electrodes of the four pads, which are connected to a signal generator. The distance between either of the parallel electrodes to its closest pin electrode is $8 \mu \mathrm{m}$.

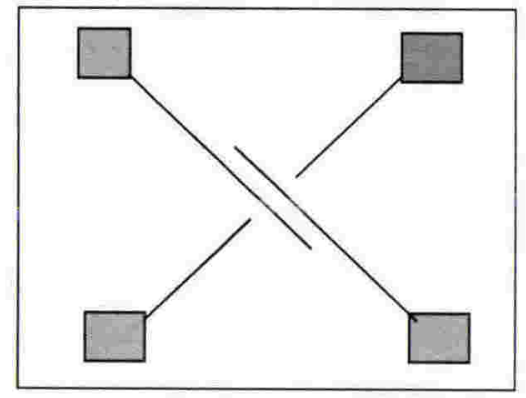

Figure 6-3 Scheme of a 4-pad structure, which was used to align single-walled CNTs.

\section{Alignment of CNTs by a 4-probe microstructure}

As mentioned in Chapter 3, single-walled carbon nanotubes were purchased from C@Rice and their Raman spectra had been given in Figure 3-14. A $5 \mu \mathrm{L}$ suspension of single-walled carbon nanotubes in distilled water with a working concentration of $100 \mathrm{ng}$ SWNT per mL, assisted by $1 \%$ SDS, was applied on top of the 4-pad structure. A sinewave voltage of 16.67 volts at $13.6 \mathrm{MHz}$ was supplied between two adjacent electrodes. The structure after alignment was observed by HRSEM.

\subsection{Results and Discussion}

The accumulation of CNTs was followed by optical microscopy, and also electrically, as a function of time. A measurement frequency of $185 \mathrm{kHz}$ was chosen as being relatively free of electrode artifacts and free of measurement spurii caused by the presence of residual $4.15 \mathrm{MHz}$ collection voltage. Depending on the method used to prepare the CNTs, two different sorts of behaviour were observed. 
Chapter 6 Alignment of Carbon Nanotubes by Dielectrophoresis

In addition, based on the real-time impedance changes of CNTs during the alignment, the effects of different solvents on the DEP behaviour of CNTs were compared.

\subsubsection{Optical microscopy images and dielectric spectrometry}

An optical microscope was used to image the real-time changes during the collection process. However, due to the limit of the optical microscope, it is impossible to view how the individual tubes respond to the electric field. The optical images presented here, as seen in Figures 6-4 (a), 6-5 (a) and 6-6, only show the behaviour of the bulk bundles of samples to the electric field.

A real-time video clip of the dielectrophoresis process was captured and presented in the oral examination.

From the optical images shown in Figures 6-4 (a), 6-5 (a) and 6-6, black threads between the electrodes appear in all the IDEs used in the experiments, despite their individually different layouts. Both of the multi-walled CNTs from the two precursors, oriented along the direction of the strongest electric field on various types of IDEs.

It can also be seen from the optical images that the collection and alignment across each gap were not uniform. The non-uniformity can be attributed to the accumulating bridging effect of conductive CNTs, which were lined up between the electrodes. These CNTs, which were attached on the electrodes, played a role as extruding electrodes while they were charged. Hence, the gap between the electrodes was shortened and the electric field intensity was increased as the alignment process went on. As more CNTs were accumulating on the electrodes, the gaps were decreased, resulting in locally increased electric field intensity. As a result, the dielectrophoretic force kept increasing between the 
electrodes with accumulating CNTs and brought more rapid and heavier collections of CNTs.

\section{Tubes from ferrocene catalyst}
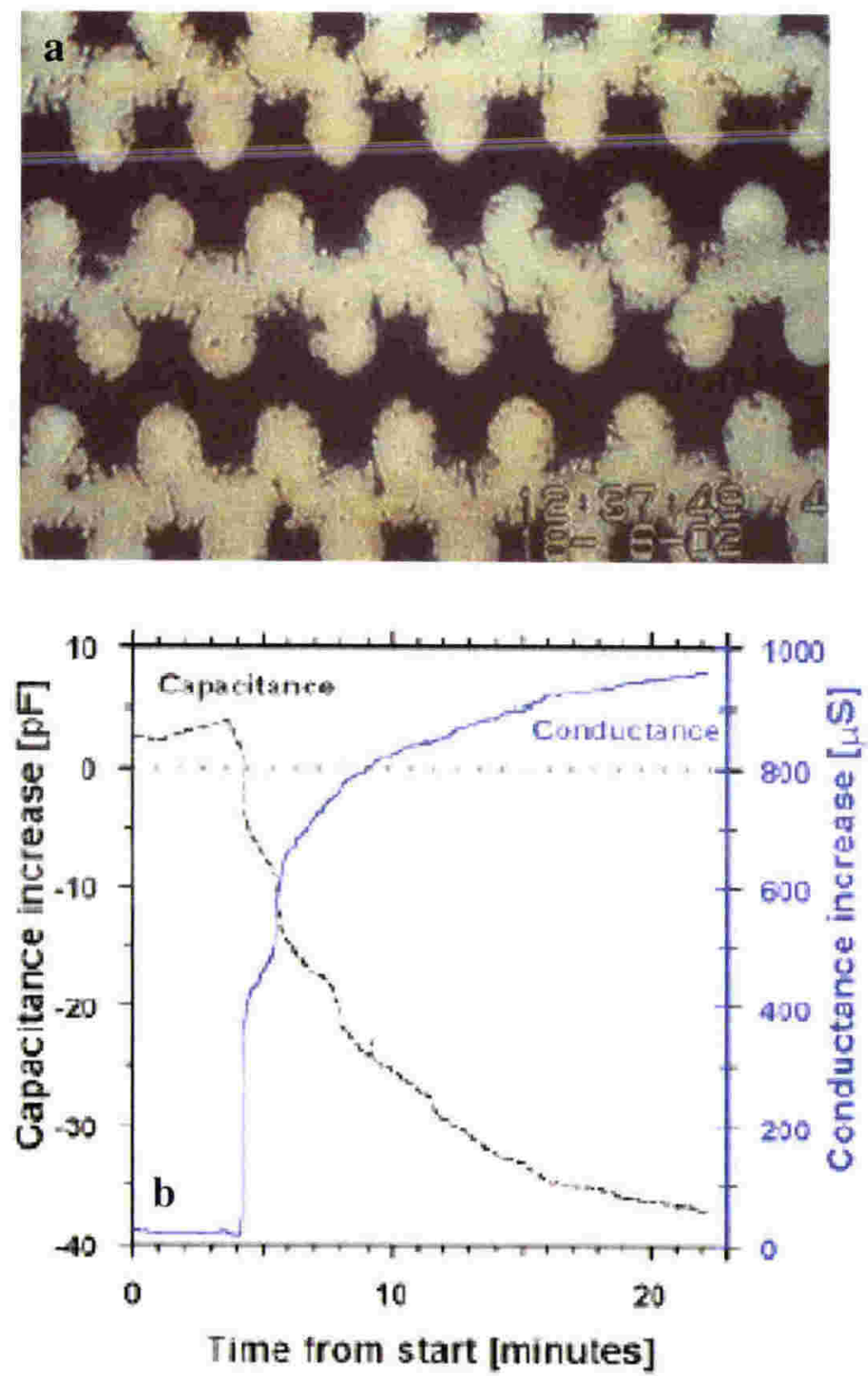

Figure 6-4 (a) Optical microscope image of CNTs from ferrocene-derived catalysts collected between electrodes at 8'07' ' after the start. The electrodes are in black and the gaps between electrodes are in white. The black aggregates are CNTs collected. The gaps between electrodes in the IDE shown in the photo are $35 \mu \mathrm{m}$ average, (b) Realtime changes during the collection as seen in (a). 
Chapter 6 Alignment of Carbon Nanotubes by Dielectrophoresis

In the case of MWNT prepared from a ferrocene-derived Fe catalyst, bridging of needlelike samples between opposite electrodes was observed after the electric field was switched on. Also, almost all of these samples were aligned along the direction of the shortest distance between the electrodes, in another word, the direction of the strongest electric field, as seen in Figure 6-4 (a).

Figure 6-4 (b) shows the plots of the real-time impedance change between the electrodes during the alignment process. In these plots, after an initial 4 minutes, the conductance rose sharply from $6 \mu \mathrm{S}$ to $400 \mu \mathrm{S}$ in less than a minute. The initial interval was an accrual period during which the bridging of conductive CNTs was not yet established. The subsequent rapid rise of the $\mathrm{AC}$ conductance can be attributed to the initial bridging of conductive samples across the electrodes. The conductance continuted to rise to $967 \mu \mathrm{S}$, together with an apparent reduction in capacitance. These phenomena suggest the occurrence of highly metallic bridge(s) of CNTs across the electrode gap.

\section{Tubes from Iron (II)-Phthalocyanine Catalyst}

When suspensions of MWNT prepared from FePC catalysts were subjected to the radio frequency field, the IDE capacitance began to increase at once. This trend continued for tens of minutes (for typical plots, see Figures 6-5 (b) and 6-5(c)). The IDE conductance was usually observed to be increasing with time, however, sometimes showed an initial, temporary decrease (visible in Figure 6-5 (c) only). The increases in capacitance and AC conductance accompanied the appearance of hair-like structures in the high-field regions of the array, which eventually became the visible bundles in Figure 6-5 (a). 


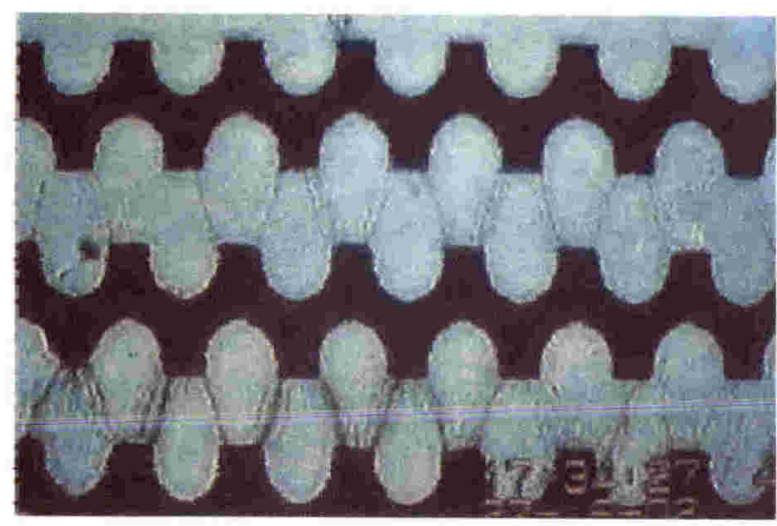

a
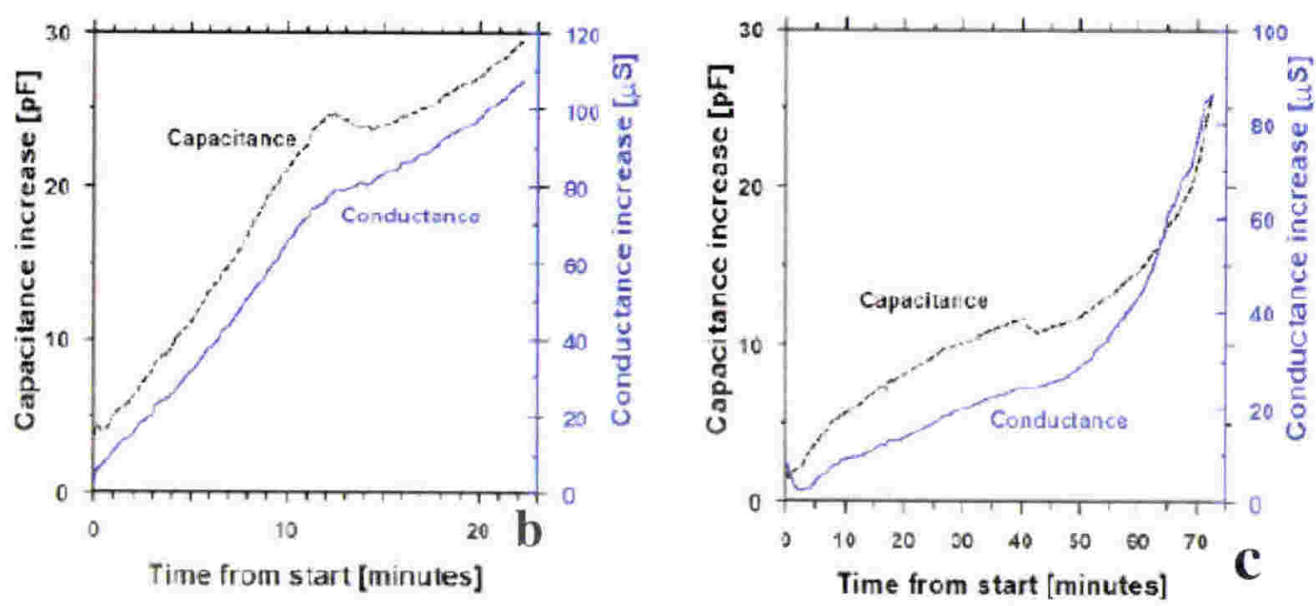

Figure 6-5 (a) Optical microscope image of CNTs from FePC-derived catalysts collected between electrodes on an IDE 1. The electrodes are in black and the gaps between electrodes are in white. The gaps between electrodes in the IDE array shown are $35 \mu \mathrm{m}$ in average; (b) Real-time impedance changes in 24 minutes during the collection as seen in (a); (c) Real-time measurement of impedance in an extended period of 72 minutes.

\subsubsection{Further optical microscopy observations and SEM investigation}

\section{Optical microscopy on more microstructures}

More IDE microstructures, IDE 2 and IDE 3, were used to align CNTs from FePCderived catalyst. Shown in the optical microscopy images in Figure 6-6, similar results of alignment with that in Figure 6-5 (a) were achieved. The two images in Figure 6-6 further suggested that the CNTs were orientated along the direction of the strongest electric field, 
regardless of the geometric layout of the IDE used. This is in consistent with the theoretical predictions of dielectrophoresis discussed in Chapter 5.
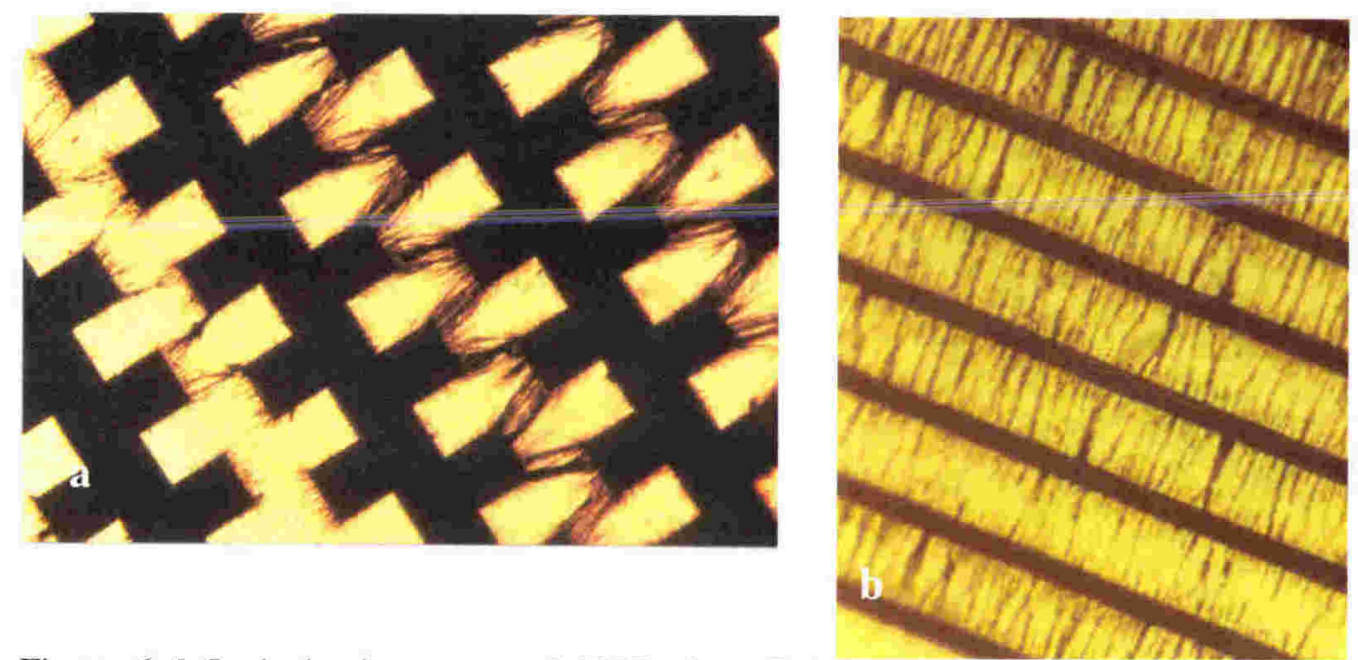

Figure 6-6 Optical microscopy of CNTs from FePC precursors collected on IDEs with different layouts, IDE 2 (a) and IDE 3 (b). In (a), Nanoutbes aligned with an operation condition as 30 peak volts between the closest gaps of $150 \mu \mathrm{m}$. In (b), CNTs aligned with operation condition as 6.0 peak volts over gaps of $40 \mu \mathrm{m}$.

\section{The response of accumulated CNTs to on/off switching of an electric field}

The impedance responses and the collection of CNTs between electrodes to the switching on/off of the AC field are shown in Figures 6-7 and 6-8. The time response experiment was conducted on an IDE 2 structure. A good correlation was found between the increases in $\mathrm{AC}$ impedances and the accumulations of CNTs observed by optical microscopy. The changes of the electrical signals observed could not have been due to heating or some electromechanical distortion of the IDE, because measurements on nanotube-free solvent revealed voltage-dependent responses less than $0.1 \%$ of those reported here.

By combining the real-time observations in Figures 6-7 and 6-8, we can deduce that the following sequence of events happened during the alignment process of CNTs: 
Initially, both the conductance and capacitance of the CNT array increased (Figure 6-7) as CNTs accumulated between opposite electrodes (Figure 6-8 (a)). When the electric AC field was switched off, the accumulations of CNTs were seen to retreat (Figure 6-8 (b)) from their positions extending across the gap, while the conductance and capacitance experiencing a sudden drop (Figure 6-7).

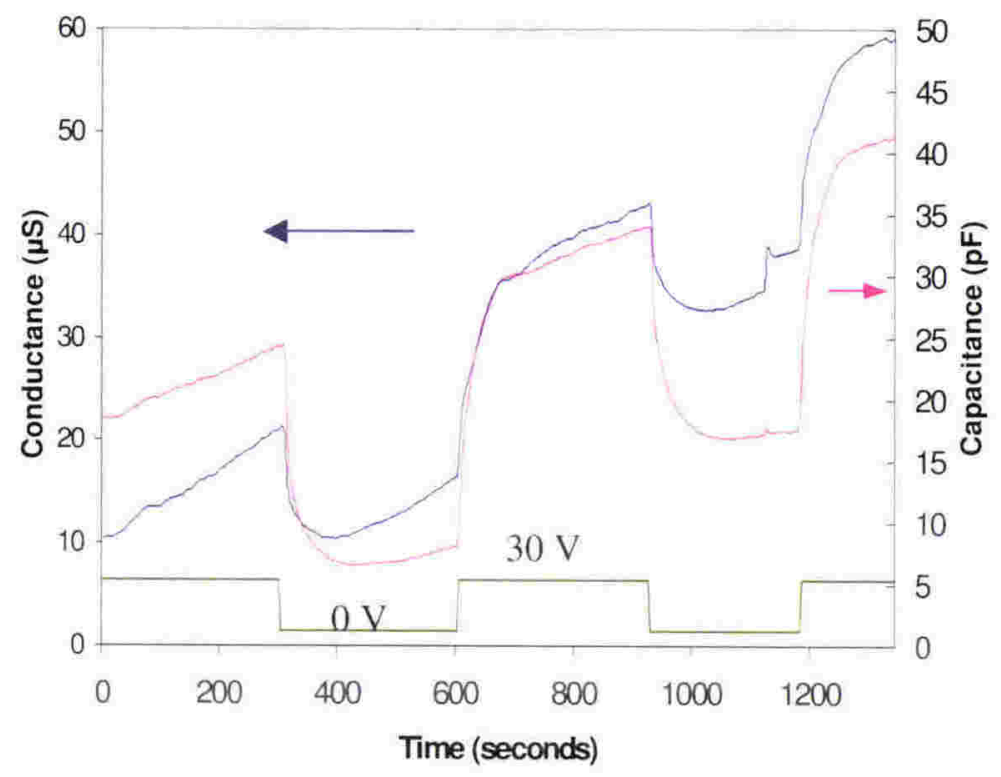

Figure 6-7 Electric field dependent behaviour of the capacitance/AC conductance of CNTs from FePC aligned on an IDE subjected to on/off switching of an electric field (30V peak across $150 \mu \mathrm{m}$, IDE 2). Time constants for conductance: 33 seconds (switch on), 15 seconds (off) and time constants for capacitances: 27 seconds (on), 13 seconds (off), with a time resolution of 3.3 seconds.

After the voltage was resumed, the conductance and capacitance increased sharply again and the bridging of CNTs was re-established (Figure 6-8 (c)). The AC impedance values, as well as the visible accumulation of CNTs, were greater than before, indicating that the overall increasing trend of the two parameters has resumed since the power was reswitched on. 
Chapter 6 Alignment of Carbon Nanotubes by Dielectrophoresis

Another observation (Figure 6-7) is that the increases in AC impedances during the second voltage-on period are approximate extrapolations of their increases during the first voltage-on period.

It can be seen that the capacitance and the conductance during the second voltage-off period are greater than those during the first voltage-off period. This phenomenon can be attributed to the continuous sedimentation of CNTs on the electrode after the start of the experiment, even when the power was off.
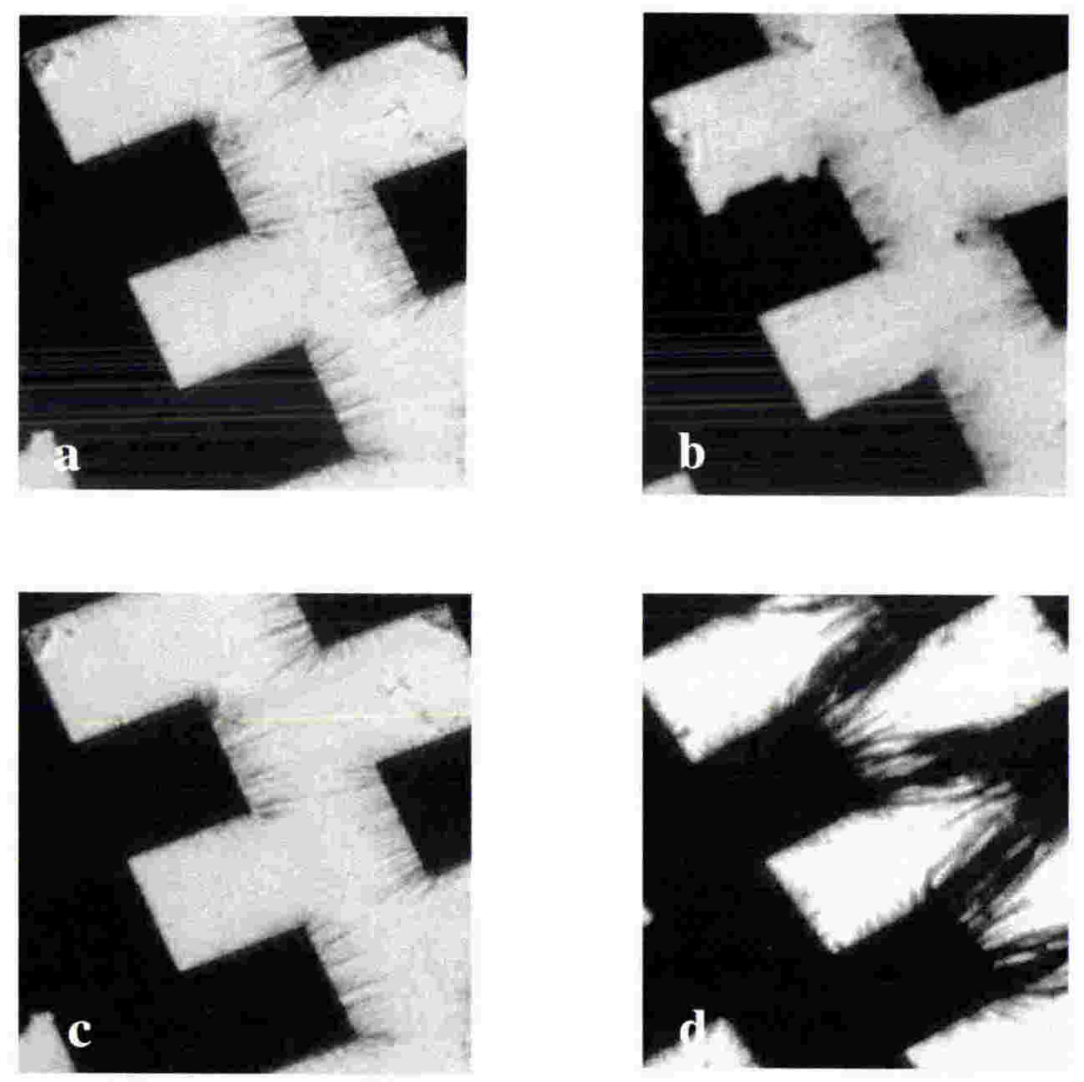

Figure 6-8 Optical micrographs of CNTs at different stages of alignment between electrodes on IDE 2, in which the gaps between electrodes are $150 \mu \mathrm{m}$ : (a) 30 seconds after application of RF voltage; (b) 15 seconds after voltage switched off; (c) 30 seconds after voltage reapplied; (d) At the end point of the alignment.

A substantial accumulation of electric field-collected CNTs was achieved after the 20th minutes in the DEP process. Figure 6-8 (d) shows that heavily accumulated CNTs were 
Chapter 6 Alignment of Carbon Nanotubes by Dielectrophoresis

spotted at the end of the alignment process. The plots in Figure 6-7 also indicate that extensive bridging of conductive CNTs happened after the power was turned on for the second time in the alignment process.

\section{SEM microscopy investigation}

SEM microscopy was employed to confirm the observations obtained from the optical microscopy. The advantage of SEM over optical microscopy in magnification provided us higher-resulted images of how the CNT bundles were aligned after evaporation of the solvents. It was confirmed that CNTs had been trapped and aligned between electrodes. Shown in Figure 6-9 are SEM images of the CNT-deposited IDE structures, which were shown by optical microscopic images in Figure 6-5 (a). The IDE structure under SEM is IDE 1. It can be indicated from these SEM images that the CNTs in alignment followed the lines of the strongest electric field intensity between sharp edges of electrodes. Also in these SEM images, there are some CNTs attracted on the top and on the edges of the electrodes. Ends of some of the aligned CNTs were extended further into the inner part of the electrodes.

Most of the bundles shown in these images are parallel to the direction of the electric field. There are also CNTs and other particles in chains, bridging the electrodes. It should be mentioned here that the gaps between electrodes are $35 \mu \mathrm{m}$ and the CNTs used in the experiment are 5-15 $\mu \mathrm{m}$, therefore, the bridgings were made of at least three CNTs in series. In some heavily deposited areas (as can be seen from Figures 6-9 (d) and (f)), the chains of nanotube bundles crossed each other. 
Chapter 6 Alignment of Carbon Nanotubes by Dielectrophoresis
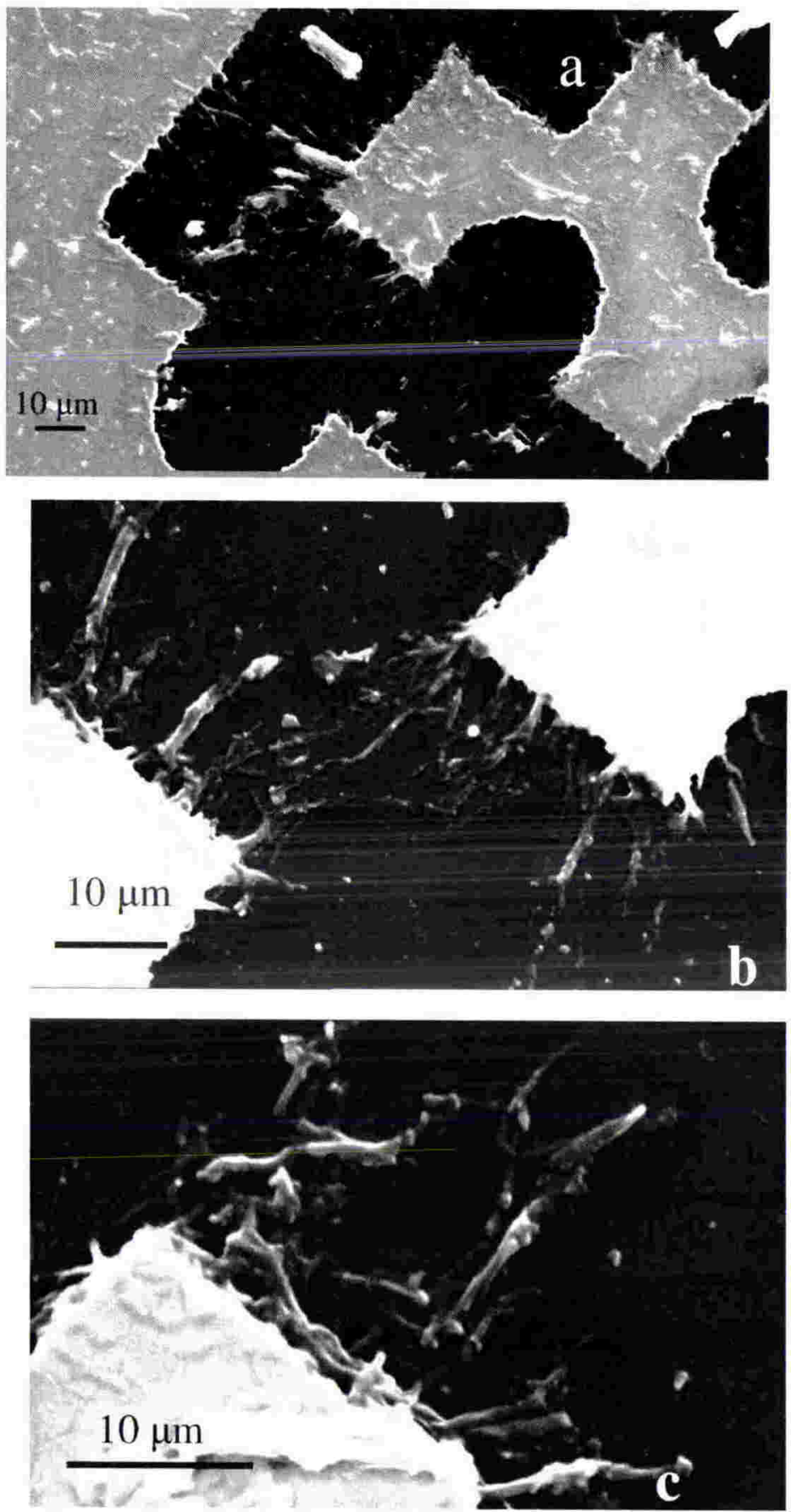

Figure 6-9 

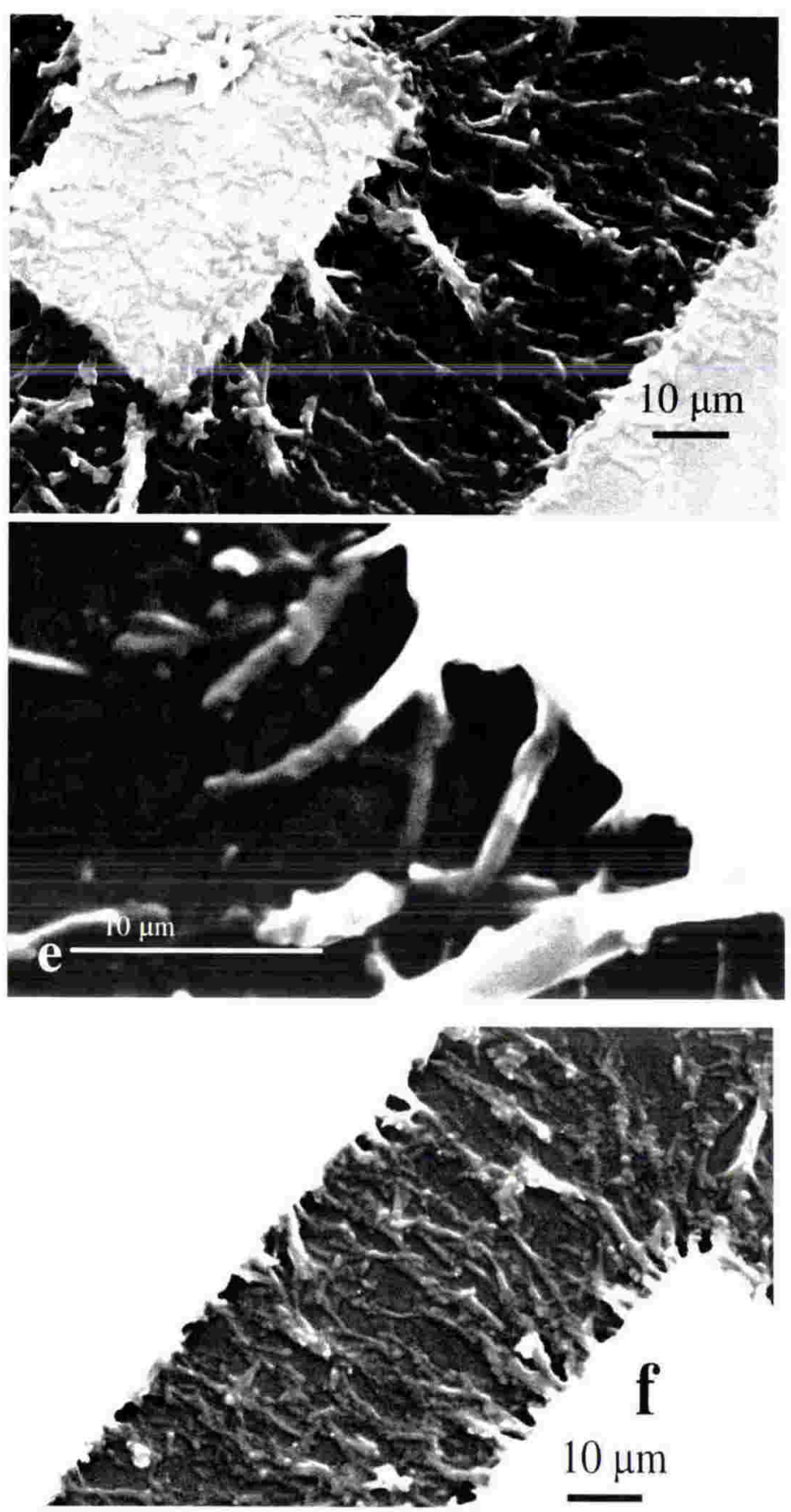

Figure 6-9 SEM images of CNTs aligned between and on electrodes of IDE 1. The electrodes are $35 \mu \mathrm{m}$ apart. The white blocks in the images are electrodes while the gaps between them are in black and CNTs are white threads. The structure used is the same as in Figure 6-5 (a).

From Figure 6-9 (e), a value of 1-2 $\mu \mathrm{m}$ can be obtained for the diameters of the individual bundles, which were lining up between the electrodes. This value is equal to the sum of 
Chapter 6 Alignment of Carbon Nanotubes by Dielectrophoresis

the diameters of approximately 25-50 CNTs, assuming that the average diameters of the CNTs are $40 \mathrm{~nm}$, as demonstrated in Chapter 3.

By comparing the optical microscopy images in Figure 6-5 (a) and SEM images in Figure $6-9$, it can be confirmed that the alignment shown in optical images actually occurred. Therefore, optical microscopy can be relied upon to give the real-time information of how CNTs are aligned on IDE structures. It can also be seen from SEM images in Figure 6-9 that there are some sections of truncated CNTs aligned between electrodes. In the SEM images on the original samples shown in Chapter 3, these broken sections were invisible. These truncated pieces might result from the ultrasonic treatment on CNT suspensions prior to the DEP.

\section{Frequency dependence of DEP orientation}

(1) The dependence of dielectrophoresis efficiency on frequency

The SEM images in Figure 6-10 demonstrated how the CNTs from FePC behave on an IDE 1 structure, with electric fields applied at different frequencies, $11 \mathrm{KHz}, 110 \mathrm{KHz}$, 1.1 $\mathrm{MHz}$ and $11 \mathrm{MHz}$. It can be drawn from the SEM images in Figure 6-10 that no alignment of CNTs was observed between electrodes at $11 \mathrm{KHz}$ (Figure 6-10 (a)) even though there were some attachments of samples on the top of the electrodes. In the experimental work in this thesis, alignment of CNTs between electrodes began to be visible in dielectroporesis experiments from a frequency of $110 \mathrm{KHz}$ (Figure 6-10 (b)). At $1.1 \mathrm{MHz}$ (figure 6-10 (c)), apparent alignment was observed between electrodes whereas at $11 \mathrm{MHz}$, the best alignment among the four frequencies was seen as Figure 610 (d). 


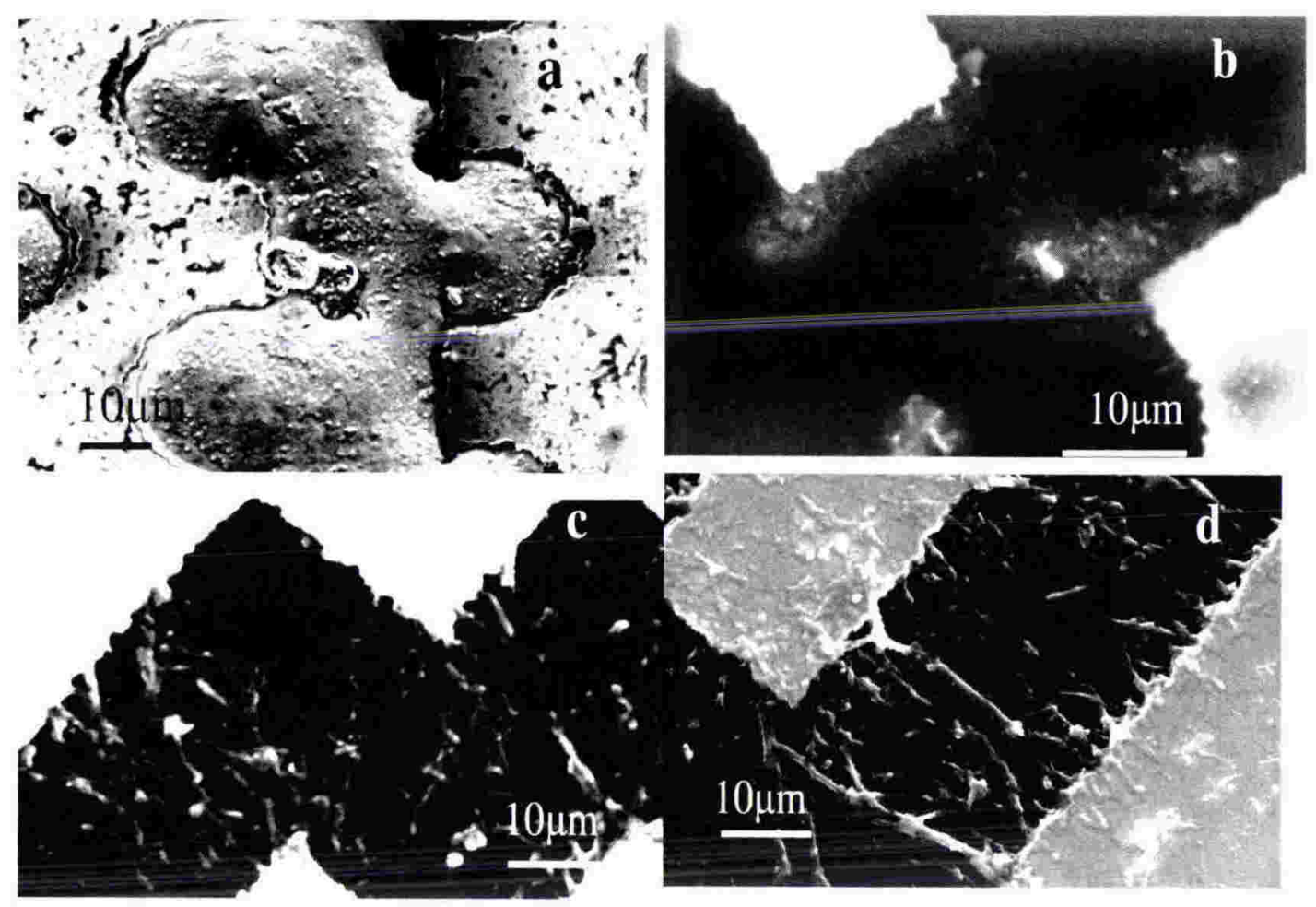

Figure 6-10 Effect of alignment of CNTs by dielectrophoresis under different frequencies. a. $11 \mathrm{KHz}$, b. $110 \mathrm{KHz}$, c. $1.1 \mathrm{MHz}$, d. $11 \mathrm{MHz}$. The layout of the IDE structure is same as the structure in Figure 6-9, IDE 1. The electrodes are $35 \mu \mathrm{m}$ apart.

A similar observation on the strong dependence of the alignment on the frequency of the electric field was reported from an independent source by Maria Dimaki et al.[5] when this thesis was drafted. However, this author did not give any further discussion on the reason of this frequency dependence behaviour.

(2) Crossover frequencies and their dependence on relative permittivity and conductivity

In DEP technique, the frequency dependence of orientation, or the crossover frequency, has been defined by Jones [6] as the frequency at which a non-symmetrical object changes their orientations in the AC electric field during a DEP process. Jones discovered that ellipsoidal objects with different relative permittivity and conductivity values have 
different effects in their DEP behaviours and crossover frequencies. Cited from reference [6], Table 6.1 listed different DEP and orientation behaviours of homogeneous ellipsoids in an AC electric field.

Table 6.1 Orientations and crossover frequencies going from low to high frequencies for a homogeneous ellipsoid in an AC electric field. $\varepsilon_{1}, \varepsilon_{2}, \sigma_{1}$ and $\sigma_{2}$ represent permittivities and conductivities of the object and the media, respectively. + DEP and -DEP mean positive and negative DEP, respectively. In the table, orientation a is along the longest axis of the object while orientations $\mathrm{b}$ and $\mathrm{c}$ are parallel to the second longest and the shortest axis of the ellipsoid. Reprint from reference [6].

\begin{tabular}{lll}
\hline & $\sigma_{2}>\sigma_{1}$ & $\sigma_{2}<\sigma_{1}$ \\
\hline$\varepsilon_{2}>\varepsilon_{1}$ & $a, a \rightarrow b \rightarrow a$, or $a \rightarrow b \rightarrow c$ & $a \rightarrow c \rightarrow b \rightarrow a$ (-DEP @ low \\
& $\rightarrow b \rightarrow a(+D E P @$ all freq.) & freq. \& +DEP @ high freq.) \\
\hline$\varepsilon_{2}<\varepsilon_{1}$ & $a \rightarrow b \rightarrow c \rightarrow a(+D E P @$ low & a or $a \rightarrow c \rightarrow a$ (-DEP @ all \\
& freq. \& -DEP @ high freq.) & freq.) \\
\hline
\end{tabular}

In terms of MWNTs and the solvent we used in the experiment, the conductivity and the permittivity of the former are higher than those of the latter. As reviewed in Chapter 5, the permittivities of MWNTs and metallic SWNTs are a large number while semiconducting SWNTs are around 5. Thus we should take $\varepsilon_{1}>\varepsilon_{2}$ and $\sigma_{1}>\sigma_{2}$ when we discuss the crossover frequencies of MWNTs here.

According to Table 6.1, the orientation along the longest axis of MWNTs occurs either at low frequencies or in a DC electric field [7] (optical microscopic images of their experiment were as shown in Figure 6-11, which was reprinted from reference [7] and in which the response of $\mathrm{CNT}$ alignment to a on-off-on switch cycle was given), or at high frequencies, in which our observation was made. And in middle frequencies, orientations other than the axial direction happen of a $\mathrm{CNT}$ and require a much bigger number of 
CNTs to make the side-to-side alignment across the electrodes. This scenario failed to happen in our experimental setup, probably because such side-to-side chains of CNTs need a much higher gradient electric field. Hereby we propose the $1.1 \mathrm{MHz}$ was over and $110 \mathrm{KHz}$ was below the crossover frequency for our Triton -assisted suspension of MWNTs from FePC in distilled water.
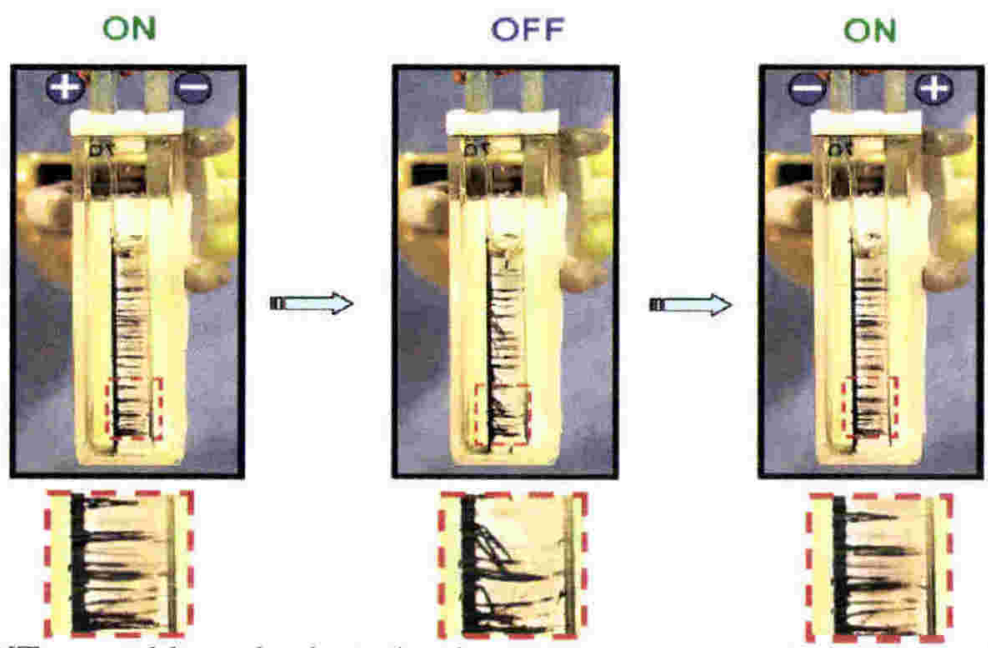

Figure 6-11 SWNT-assembly and orientation in response to an applied de electric field in THF. The left to the right sequence corresponds to an on (+) -off-on (-) cycle of the field. Reprinted from reference [7].

\section{(3) A frequency scan after the solvent was evaporated}

A frequency scan was conducted on CNT samples from FePC precursors after the alignment process was finished and the solvent was dried. The plots of admittances versus frequency in Figure 6-12 showed a strongly frequency-dependent capacitance and conductance between $10 \mathrm{kHz}$ and about $0.4 \mathrm{MHz}$. It is noted from the plots in Figure 6-12 that the capacitance obeys a $(1 / \mathrm{f})^{\alpha}$ law and the conductance increases with the increasing frequency. Such a phenomenon does not appear to be conduction-dominated charge transfer behaviour. 


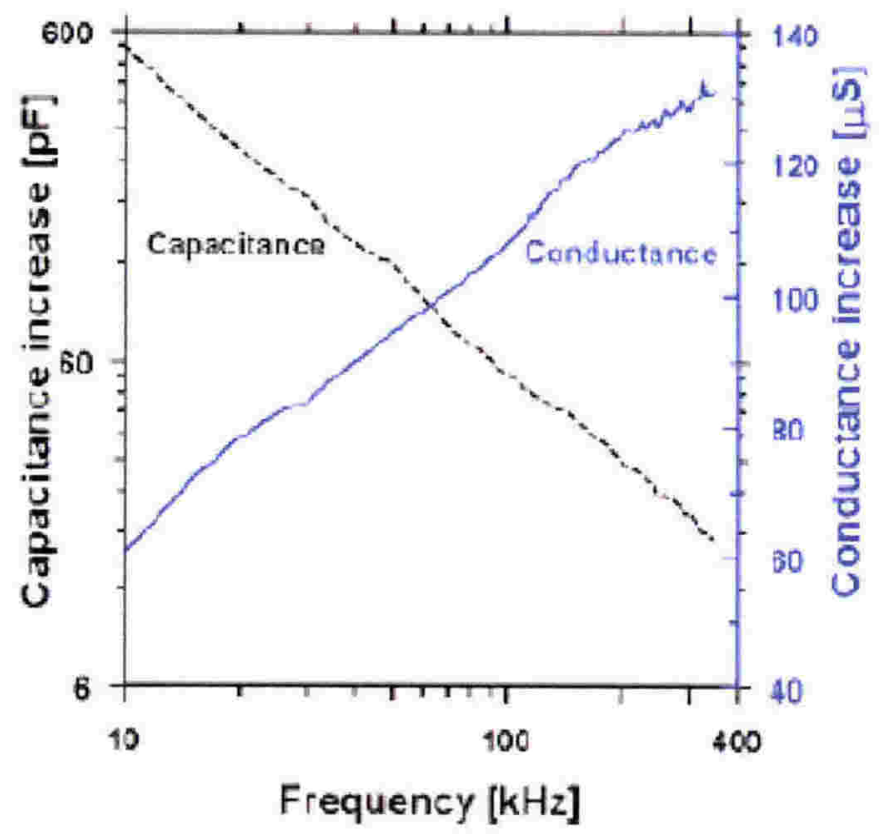

Figure 6-12 Frequency scan of capacitance and conductance of collection of CNTs from FePC catalyst on IDE 1. The structure used is as seen in Figures 6-6 (a) and 6-9.

\subsubsection{Comparison of the DEP behaviour between two types of CNTs}

Compared with the observation with CNTs with ferrocene-derived catalyst, there are some notable discrepancies from that with FePC-derived CNTs: first, the capacitance increases during the alignment process; second, the initial pause and sharp increase of conductance did not occur; third, accumulation of CNTs between electrodes is much more smooth.

Accumulations of those CNTs formed from FePC-derived catalyst were slender and highly field-oriented (Figures 6-5(a) and 6-8). These caused a progressive increase in both admittance components, but no apparent ohmic contact. Rather, the $1 / \mathrm{f}$ behaviour of the capacitance (Figure 6-12) is consistent with an interfacial-type of polarisation mechanism. It appears that the current path to/from these CNTs was through at least a thin layer of liquid medium, rather than by direct contact. 
By contrast, MWNTs formed on ferrocene-derived Fe gave rise to denser, more compact aggregations on the electrodes (Figure 6-4 (a)), and these seemed capable of forming "bridging" paths, with highly conductive electrode-nanotube contact. This resulted in a reduction in the capacitance signal (Figure 6-4 (b)). It may be that the reason for this difference lies in the pronounced differences in tube thickness and folding between these preparations, or else in intrinsic conductivity differences caused by different levels of iron content. Both of these two factors are considered to be possible conducting channels.

\subsubsection{Solvents}

Table 6.2 gives the comparisons of the capacitance and conductance changes measured at 4.15 MHz among suspensions of CNTs in distilled water, ethanol or isopropanol during electric-field orientation. The two parameters were chosen to show the effect of dielectrophoresis because they indicated the bridging of CNTs across the electrodes.

Table 6.2 Comparison of the conductance and capacitance increases observed when CNTs $(15 \mu \mathrm{g} / \mathrm{ml})$ suspended in distilled water, absolute ethanol and absolute isopropanol were subjected to alignment by electric field.

\begin{tabular}{|c|c|c|c|}
\hline & Relative & Conductance & Capacitance \\
Solvent & Permittivity & increase & increase \\
& $\left(20^{\circ} \mathrm{C}\right)[4]$ & $\left(\times 10^{-6} \mathrm{~S}\right)$ & $\left(\times 10^{-12} \mathrm{~F}\right)$ \\
\hline Distilled water & 80.1 & $44-195$ & $20-67$ \\
\hline Ethanol & 25.3 & $8-10$ & $2.5-8$ \\
\hline Isopropanol & 20.2 & $0.8-6.0$ & $0.1-1.4$ \\
\hline
\end{tabular}

The measurements on conductance and capacitance were taken four times for each solvent. CNTs suspended in distilled water yielded at least five times greater changes 
both in conductance and capacitance than the CNTs in the other solvents used. Therefore, for a surfactant-assisted suspension system, distilled water is the best candidate among the three solvents in orientation experiments. This is consistent with the fact that raising the permittivities of the solvent should increase electric-field-induced forces [9], as shown in Equation 5-7.

\subsubsection{Aligning Single-walled Carbon Nanotubes with a 4-electrode} Structure
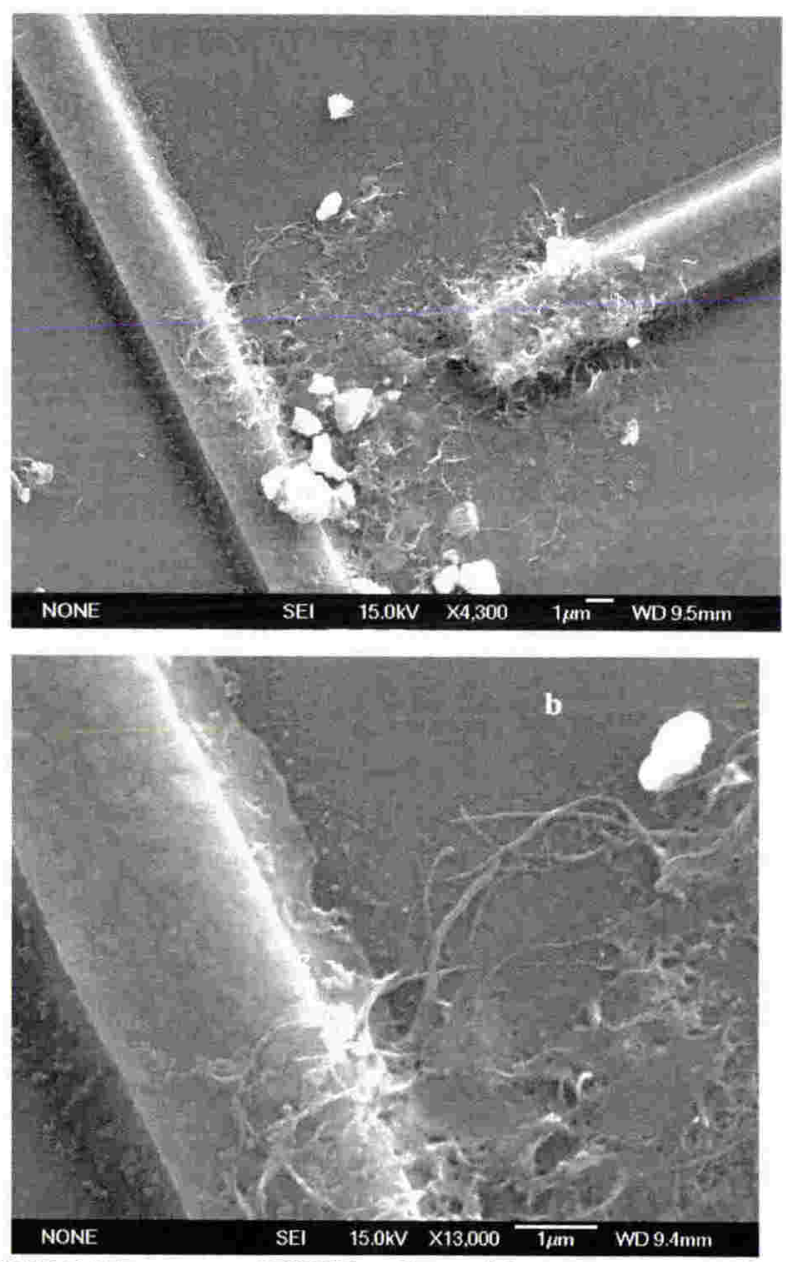

Figure 6-13 HRSEM images of CNTs aligned between two adjacent electrodes on a 4-pin structure. (a) overview on the area between two electrodes, (b) magnified view of CNTs on one of the electrodes. 
Alignment of SWNTs was observed on the 4-pin microstructure. A sine-wave voltage of 16.67 volts at $13.6 \mathrm{MHz}$ for alignment was applied between one of the parallel electrodes and its adjacent pin electrode. HRSEM images of SWNTs on the structure after distilled water was completely evaporated are shown in Figure 6-13. The SWNTs followed the orientation of the strongest electric field between the electrodes and some of those were attached to the electrodes. It should be noted that the intensity of the electric field here on the 4-pin layout is much stronger than the electric field, which was exerted on the IDEs in the alignment experiment in the previous sections because of the closer gap between electrodes and the sharp configuration of one of the electrodes in the 4-pin microstructure. From these images, the SWNTs in bundles with diameters of approximately $150 \mathrm{~nm}$ can be seen aligned between the electrodes. There are also some crystallized SDS surfactants, which can be observed from the HRSEM images.

From the above observations, we can draw a conclusion that both the IDE and the 4electrode structures can be used to collect CNTs between the electrodes in an AC electric field at an appropriate frequency.

The results discovered in this chapter can be extended to device fabrications: metallic CNTs can be trapped between electrodes from suspensions using the DEP technique mentioned in this chapter to yield metal-CNT-metal structures, for example, CNT-based interconnects [8], which are able to sustain much higher electric current than their copper counterparts. Besides, IDE electrodes can be used as "microtweezers" to carry and drop conductive CNTs with the on/off switching technique mentioned in this chapter. CNTs 
can be delivered into desirable places during the processing of CNT-based devices by controlling the switches of the external circuits, or by alternatively applying voltages.

\section{Reference:}

1. W. M. Arnold. Institute of Physics Conference Series, p. 63-68, 1999.

2. W. M. Arnold. Annual Report Conference on Electrical Insulation and Dielectric Phenomena, IEEE2001, p. 40-43, 2001.

3. W. M. Arnold and G. C. Turner. Annual Report Conference on Electrical Insulation and Dielectric Phenomena, IEEE1998, p. 360-363, 1998.

4. K. D. Ausman, R. Piner, O. Lourie, and R. S. Ruff (2000), The journal of Physical Chemistry B, 104: p. 8911-8915.

5. M. Dimaki and P. Boggild (2005), Nanotechnology, 16: p. 759-763.

6. T. B. Jones (1995), in book. Electromechanics of Particles Cambridge: Cambridge University Press

7. P. V. Kamat, K. G. THomas, S. Barazzouk, G. Girshkumar, K. Vinodgopal, and D. Meisel (2004), Journal of American Chemistry Society, 126: p. 10757-10762.

8. F. Kreupl, A. P. Graham, M. Liebau, G. S. Duesberg, R. Seidel, and E. Unger (2004), eprint arXiv:cond-mat/0412537.

9. H. A. Pohl, in Book Dielectrophoresis: The Behavior of Neutral Matter in Nonuniform Electric Fields. 1978, Cambridge University Press: Cambridge. p. 579 . 


\section{Chapter 7}

\section{Selective Purification of Multi-walled CNTs by Dielectrophoresis within a Large Array}

Since the discovery of CNTs [8], remarkable progress has been made in the synthesis, characterisation and application aspects of these novel materials. As described in Chapter 4, several dry or wet methods $[2,4,6,16]$ for purification and selection of CNTs have been reported. However, no effective large-scale methods have yet been found for the removal of as-grown impurities, such as carbon nanoparticles or amorphous carbon, without damaging the structure of CNTs. Recently, progress has been made in positioning CNTs by means of electric fields. As reported earlier [3, 17] and discussed in Chapter 6, the high conductance and therefore the high polarisability of the CNTs enable them to be aligned and collected from their dispersion in a liquid onto electrodes across which sufficient radio-frequency voltage is applied. Furthermore, this phenomenon can be utilized to separate the pseudo-one dimensional CNTs from catalyst-derived and carbon-related impurities in suspensions.

The phenomenon utilized here for separation of MWNTs from as-grown impurities is dielectrophoresis [13], which describes the movement of neutral but polarisable particles in a non-uniform electric field as mentioned in Chapter 5 and has been used for aligning MWNTs from suspensions in Chapter 6. 

Array

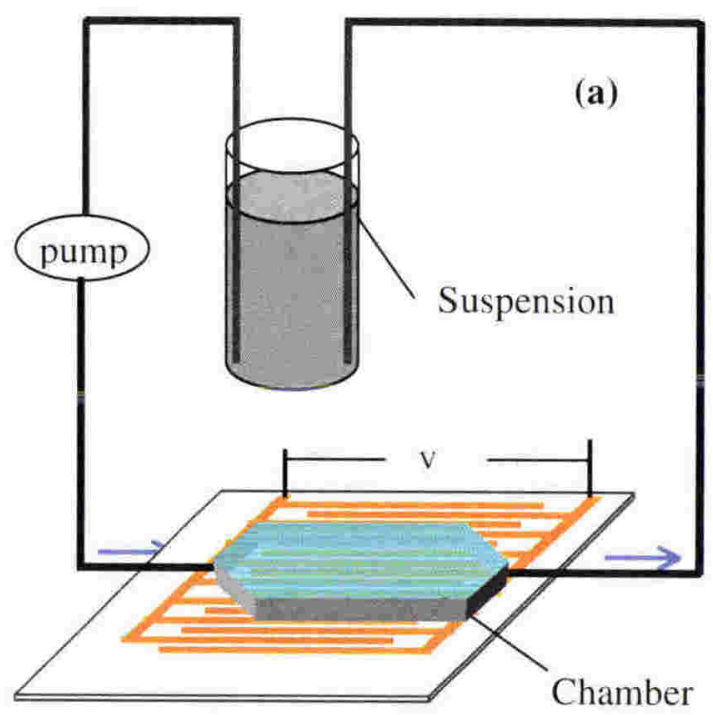

(b)

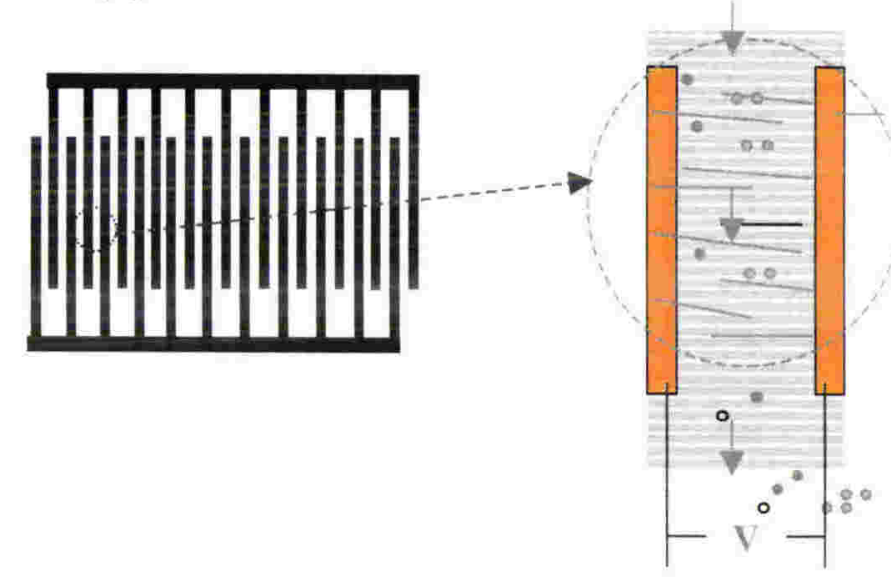

Figure 7-1 Apparatus of the prototype device which aims to selectively purify as-grown CVD-made MWNTs. (a) Diagram of the apparataus, which includes an array with interdigitated electrodes, an AC power source (not included in this diagram), a pump and a chamber which confines the flow of MWNTs suspension; (b) Schematic representation of the selection process. The right side is the magnified diagram of the interdigitated electrodes as depicted on the left side. The solid arrows represent the flow direction of the suspension. The solid rods indicate MWNTs. The solid circles and the empty circles indicate catalyst-related impurities and carbon-related impurities, respectively.

The previous chapter has described our results of aligning CNTs by the DEP technique. Applying a non-uniform electric field at a high frequency into well-dispersed CNTs 
Chapter 7 Selective Purification of Multi-walled CNTs by Dielectrophoresis within a Large Array

suspensions generates a large dielectrophoretic force upon the CNTs, and as a result, this force will align CNTs along the electric field vector. In addition, compared with spherical nanoparticles impurities in as-grown nanotube samples, CNTs are much more susceptible to the dielectrophoretic force and can be selected from the as-grown impurities. In this chapter, dielectrophoresis technique was used for the purpose of selection of CNTs, and the related results are presented.

Based on the results presented in the previous chapter, an array with a size of $141 \mu \mathrm{m} \times$ $193 \mu \mathrm{m}$ was set up. The layout of the prototype device is depicted in Figure 7-1 (a). As illustrated in Figure 7-1 (b), the principle of the device is as follows: CNTs in the suspension are more susceptible to dielectrophoretic force than as-grown impurities due to their higher conductance and therefore higher polarisabilities. CNTs are collected on the electrodes by dielectrophoretic force while impurities are flushed into the recirculated suspension by hydrodynamic force exerted by a pump.

\subsection{Experimental}

\subsubsection{Samples}

\section{The source of the samples}

MWNTs we made in chapter 3 by CVD is less than $1 \mathrm{mg}$ from each run and had been used for the alignment experiment in Chapter 6. However, a large quantity of CNTs (2.25 $\mathrm{mg}$ for each run, $150 \mathrm{ml}$ suspension) are required for repeatable operations and comparison purpose in the separation experiment in this chapter, and we need identical and homogeneous supply of CNTs, which are only available from commercial samples. 
Chapter 7 Selective Purification of Multi-walled CNTs by Dielectrophoresis within a Large Array

The CNTs samples, which were to be separated, were MWNT samples and were provided by Ahwanee Technology [7].
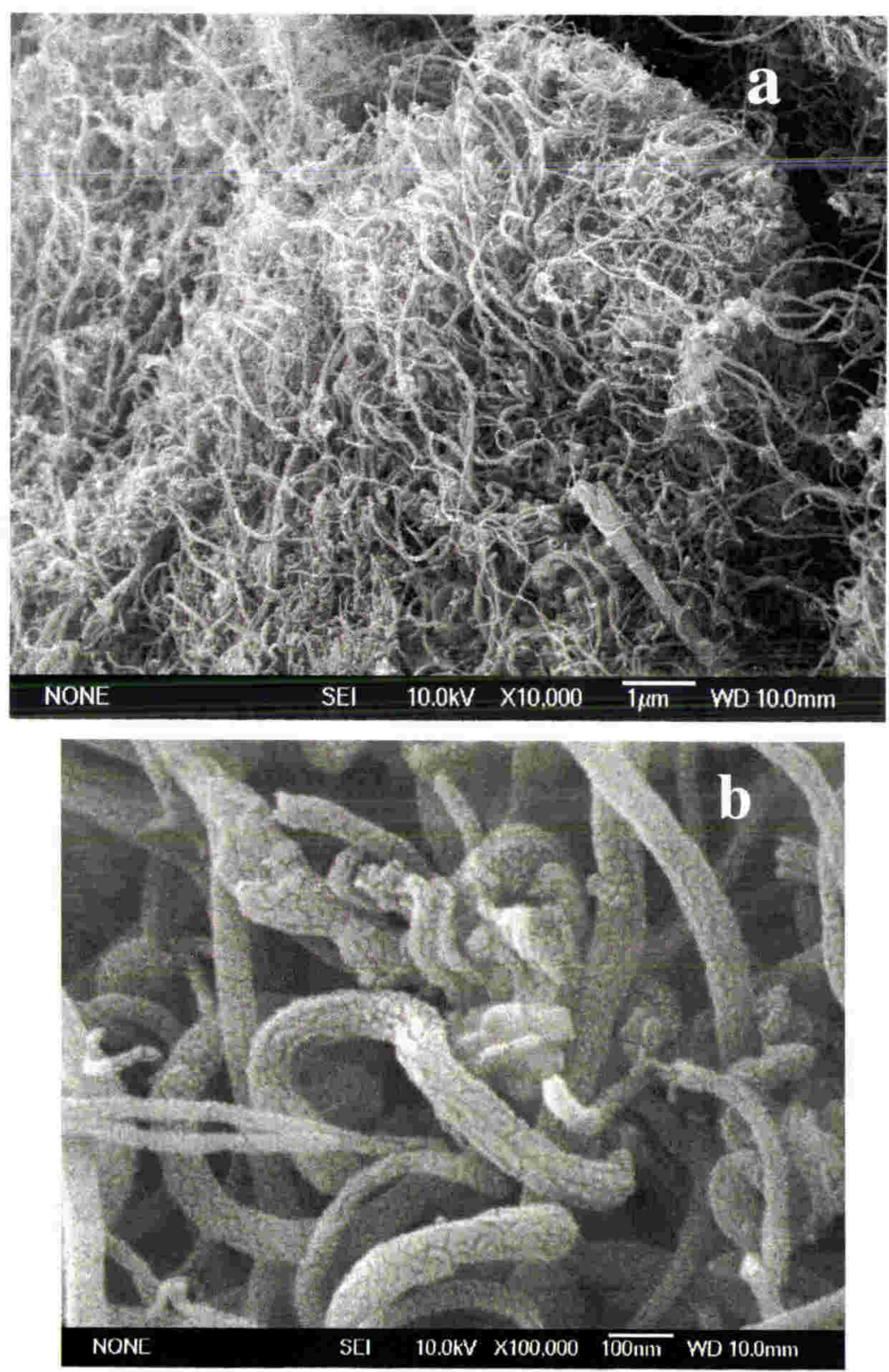

Figure 7-2 HRSEM images on MWNTs from other sources, for dielectrophoresis purpose, a. at a low resolution. b. MWNTs under a high resolution. Please note that in this image, the surfaces of nanotubes appeared to be coarse, this artefact was caused by a $4 \mathrm{~nm}$ coating of Au before HRSEM. In fact, the surfaces of nanotubes should be very smooth according to HRSEM observations without Au coating. 
Chapter 7 Selective Purification of Multi-walled CNTs by Dielectrophoresis within a Large Array

According to the provider, $94 \%$ by weight of each batch of sample was estimated to be MWNTs. The remainder of the sample was carbon-related and catalyst-derived impurities. The HRSEM images in Figure 7-2 show that the samples used are non-linear and highly entangled, and contain nanoparticle impurities. These samples were used in the experiment without any purification.

2. Nanotube Suspensions: MWNT samples were suspended by mixing them in the ratio of $1.0 \mathrm{mg}$ (MWNT sample): $1 \mathrm{~mL}$ distilled water: $15 \mu \mathrm{L}(1 \%$ Triton X-100, a non-ionic surfactant). After 30 minutes in an ultrasonic bath, every $15 \mu \mathrm{L}$ suspension was mixed with $1 \mathrm{~mL}$ distilled water, and a further $15 \mu \mathrm{L} 1 \%$ Triton $\mathrm{X}-100$. A further 15 minutes ultrasonication gave the working suspension.

\subsubsection{Apparatus}

As shown in Figure 7-1 (a), the prototype device consisted of a PCB (printed circuit board) electrode array, a polymethacrylate cover, a radio frequency power source (described elsewhere [1]) and a pump to circulate the suspension.

\section{Printed circuit array}

A printed circuit board (PC-board) separation chamber was established to combine dielectrophoretic force and hydrodynamic force together for separation of multi-walled nanotubes from as-grown nanotube suspensions. The printed circuit array, which consists of scaled-up interdigitated electrodes, as shown in Figure 7-1 (b), is the key element of the separation system, and can be considered as a scale-up structure of the IDEs, which had been used in Chapter 6 . 
Chapter 7 Selective Purification of Multi-walled CNTs by Dielectrophoresis within a Large Array

The scaled-up interdigitated electrode array was fabricated as a printed circuit board with a single-sided Cu-clad glass-epoxy laminate. The copper was then gold-plated to prevent corrosion. Electrodes were plain parallel fingers, 141 micron wide and 193 micron between each other. Of the $130 \mathrm{~mm} \times 134 \mathrm{~mm}$ array area, only about $30 \%$ was covered under the flow-chamber used for MWNT purification.

\section{Separation chamber}

The chamber was made of transparent polymethacrylate so that the suspension could be seen through it. When the chamber was pressed on the array, it sealed a $2 \mathrm{~mm}$ thick layer of liquid above it. The setup diagram of the apparatus is shown in Figure 7-1 (a). This setup allowed a re-circulating flow of the suspension of MWNTs. The main reservoir of MWNTs suspension was held in a beaker, assisted by an ultrasonic bath (not shown in the Figure) to prevent sedimentation of CNTs during the re-circulation.

\subsubsection{Set-up}

A CNT working suspension $(150 \mathrm{~mL})$, maintained by an ultrasonic water bath to prevent sedimentation, was pumped and recirculated through the chamber after the RF power was turned on. The RF power of 22.5 volts peak at a frequency of $350 \mathrm{kHz}$ was applied across the electrodes for up to 150 minutes. A multimeter (with a RF probe, connected to a computer) and an oscilloscope were employed to record the real-time current in the circuit and the voltage across the array, respectively. 
Chapter 7 Selective Purification of Multi-walled CNTs by Dielectrophoresis within a Large Array

\subsubsection{Characterisation}

After 150 minutes collection of CNTs, the array was viewed under an optical microscope with a $20 \times$ lens. A photograph of the array and two photomicrographs of the array after the collection are shown in Figure 7-3.

The MWNT samples without collection and those collected on the array were characterised by SEM and Raman spectroscopy. The samples collected on and between electrodes for SEM were removed from various areas of the array by means of carbon tapes and were mounted on SEM sample holders. Samples of the suspension (not from the electrodes) had been taken and dried before SEM.

Raman spectroscopy was carried out using a Jobin-Yvon LabRaman HR micro-Raman spectrometer with $632.8 \mathrm{~nm}$ laser excitation. A 100x objective lens was used, with a power density of $4 \mathrm{~mW}$ over 4 square microns. Spectra of the collected samples were taken in situ at 48 different spots on the array whilst the dry samples without treatment for Raman characterization were dropped on the array to obtain the same background. Raman spectroscopy of the latter sample was conducted at 30 different spots on the array. In addition, samples were taken from the suspensions at 0 th, 30th, 75th and 150th minutes (at least five samples each time) during the DEP, and were dried at $105^{\circ} \mathrm{C}$ on $\mathrm{Si}$ wafers in an oven overnight before being characterised by Raman spectroscopy.

\subsection{Results and Discussion}

The assignment $[9,10,14]$ of Raman signals has been extensively reported, as mentioned in Chapter 1. These reports suggested that in the Raman spectrum, a weak or absent peak at $1349 \mathrm{~cm}^{-1}$ (D band), means a higher graphitization degree or less disorder peak of 
Chapter 7 Selective Purification of Multi-walled CNTs by Dielectrophoresis within a Large Array

CNTs and the absence of the shoulder near the strongest peak at $1582 \mathrm{~cm}^{-1}$ (G band) means less lattice distortion.

\subsubsection{Optical microscopic and SEM images}

As shown in Figure 7-3 (b), a large amount of dark materials were collected as linear, parallel threads between the electrodes on the array. The width of these threads averaged at $10 \mu \mathrm{m}$.
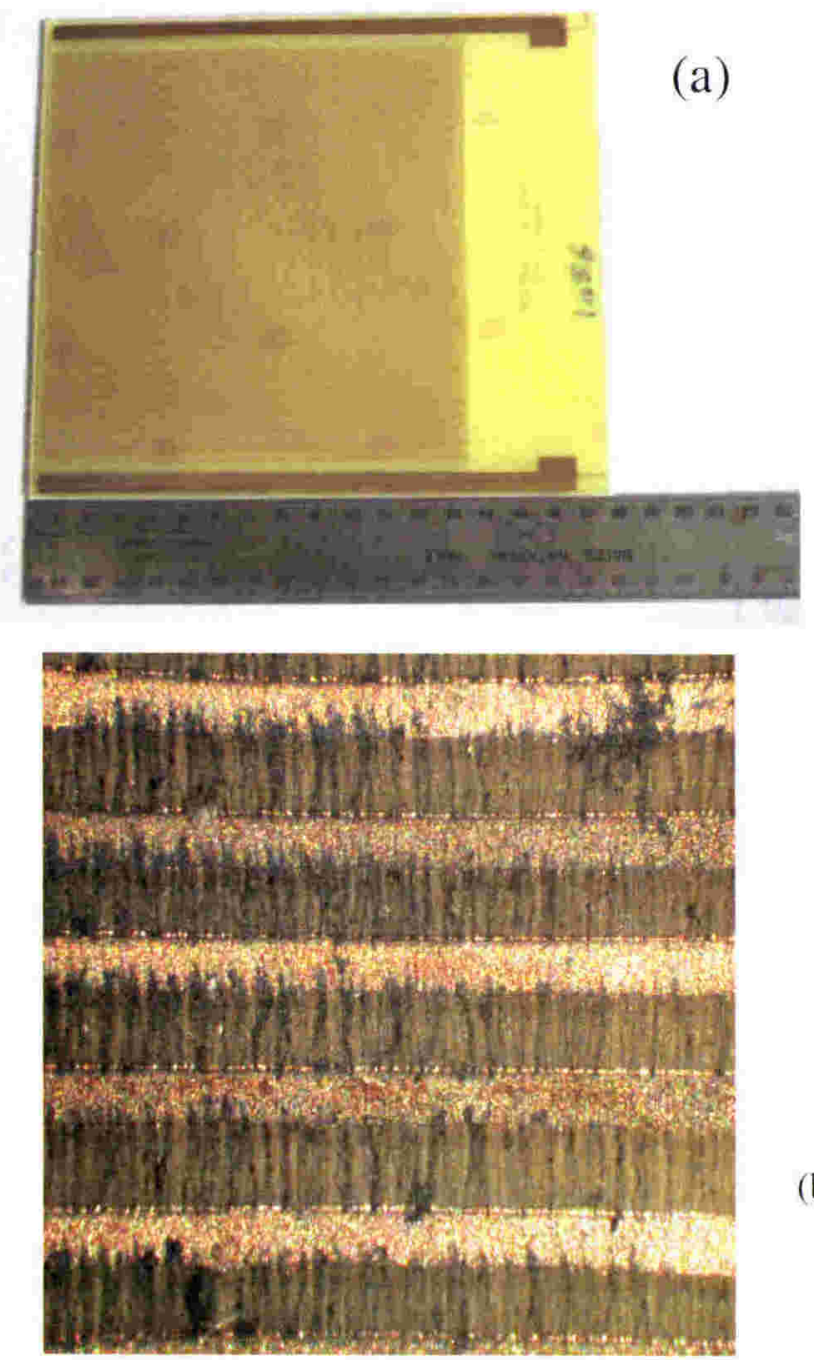

(b)

Figure 7-3 


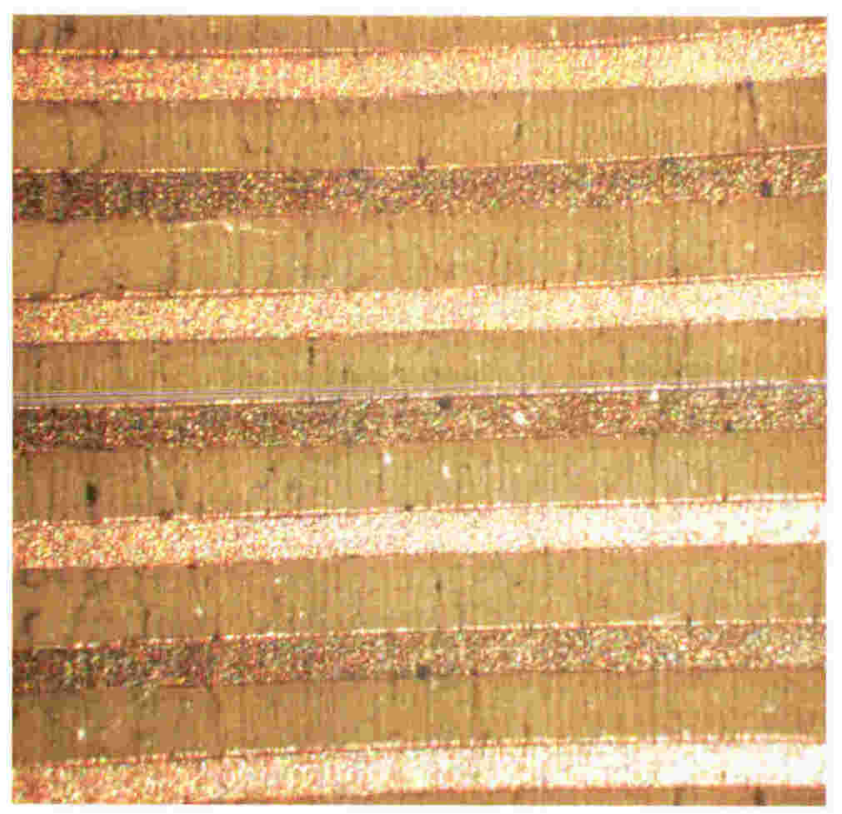

(c)

Figure 7-3 Views of the large array before and after collection of CNTs. The suspensions were pumped in from the left to the right. (a) An overall view of the array with IDEs. A ruler indicates the dimensions of IDEs: $13.5 \mathrm{~cm} \times 11.5 \mathrm{~cm}$. (b) and (c) Views, which are magnified by an optical microscope, of CNTs collected on and between electrodes. The electrodes (141 $\mu \mathrm{m}$ wide and $193 \mu \mathrm{m}$ apart) are in metallic colour while collected CNTs are black line-ups. (b) A heavily deposited area and (c) A lightly deposited area.

Although the optical microscopic image showed that the MWNT samples were packed as black threads between the electrodes, the SEM image (see Figure 7-4 (c)) of the nanotubes after dielectrophoretic collection showed that MWNTs were still in tangles, which had already existed in the MWNTs before the separation. In our previous publications $[11,12]$ and in Chapter 6 of this thesis, however, the SEM images displayed that MWNTs were aligned in straight bundles between electrodes. This difference between the current and previous results, in the morphology of MWNTs samples which were collected across the electrodes, may be attributed to two possible causes.

First, there are the different morphologies in the samples. In our previous results, the MWNTs were prepared in-house and showed straight morphology before alignment, as 
Chapter 7 Selective Purification of Multi-walled CNTs by Dielectrophoresis within a Large Array

shown in Chapter 3. However, The MWNTs we used here in this chapter from an outside provider. The latter sample was highly tangled, as demonstrated in the HRSEM images in Figure 7-2.

Second, the frequency of the electric field was different: in the previous publications [11, 12], AC electric field was applied at $4.15 \mathrm{MHz}$ in small-sized IDEs, while in this case it is necessary to limit the frequency to $315 \mathrm{kHz}$ for the scaled-up apparatus because of the electrical characteristics of the large array.
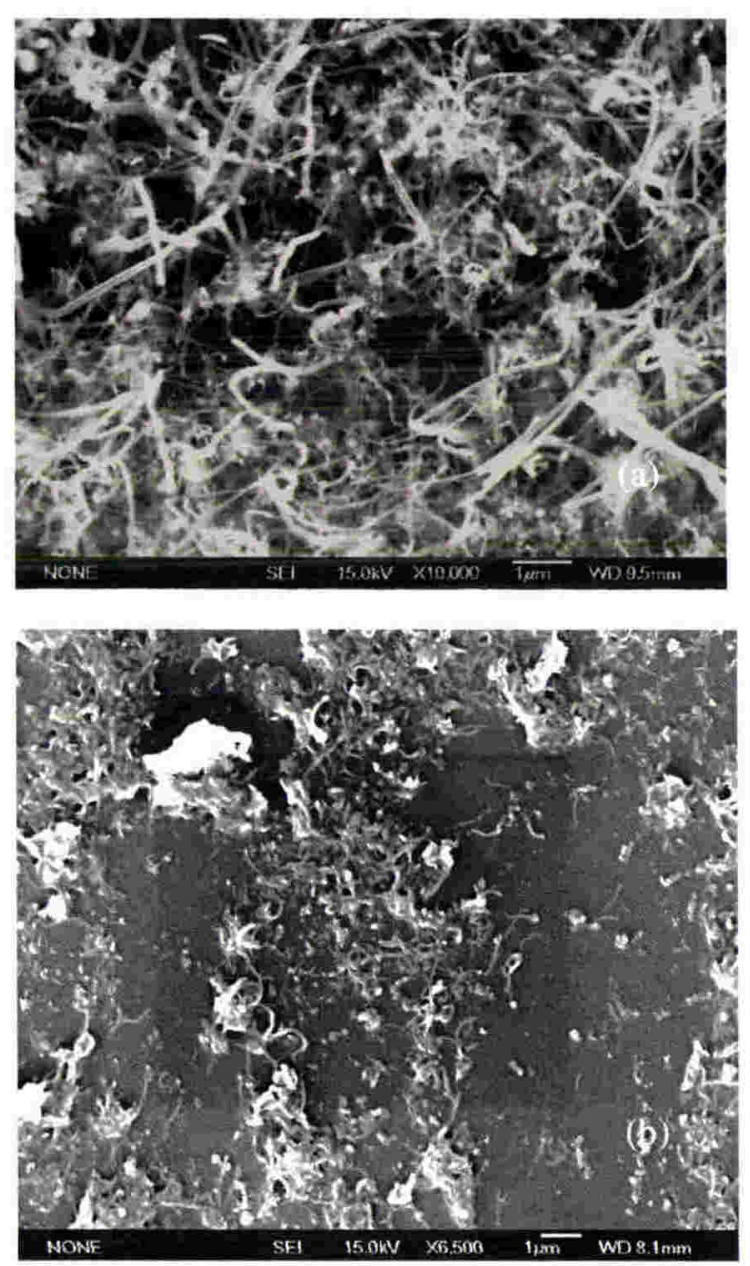

Figure 7-4 

Array

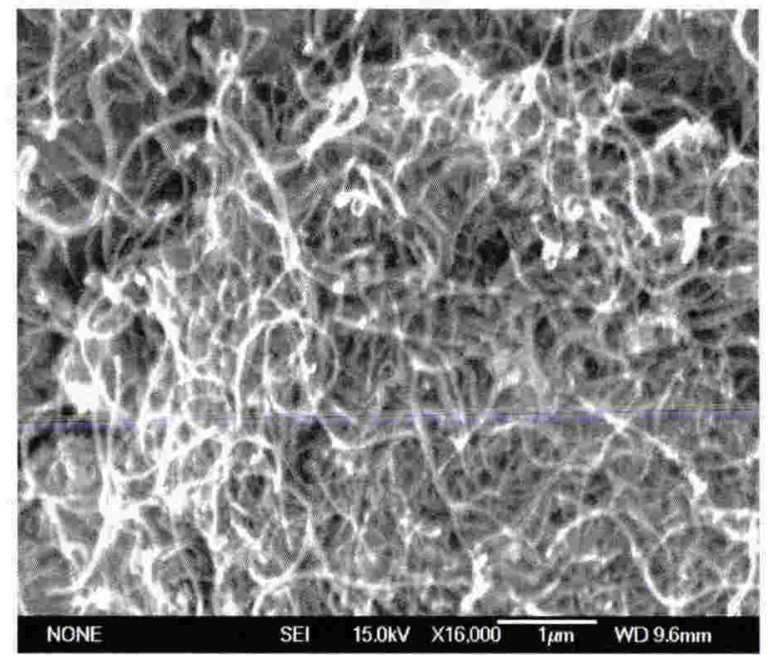

(C)

Figure 7-4 SEM images of samples (a) before being treated, (b) remained in the suspension after being treated and (c) collected on the array. All the scale bars in the images are $1 \mu \mathrm{m}$.

Comparison of the SEM images of samples in Figure 7-4 indicates that the MWNTs collected on the array, were of higher purity than those in the original sample and large amounts of impurities remained in the suspension, and above all, the impurities in the samples were substantially reduced after being treated by the prototype device.

\subsubsection{Electrical parameters}

The changes of electrical parameters of the array versus time were plotted in Figure 7-5. As seen from the plots, after sharp changes in the first 40 minutes, both the current and voltage changed relatively slowly. This phenomenon can be attributed to an initial rapid bridging of conductive MWNTs between the electrodes, followed by a slower saturation phase after the 40 th minute of the run. The accumulating CNTs caused by DEP across the electrodes are responsible for the increasing current and the declining voltage in the Figure 7-5 plots. 
Chapter 7 Selective Purification of Multi-walled CNTs by Dielectrophoresis within a Large Array

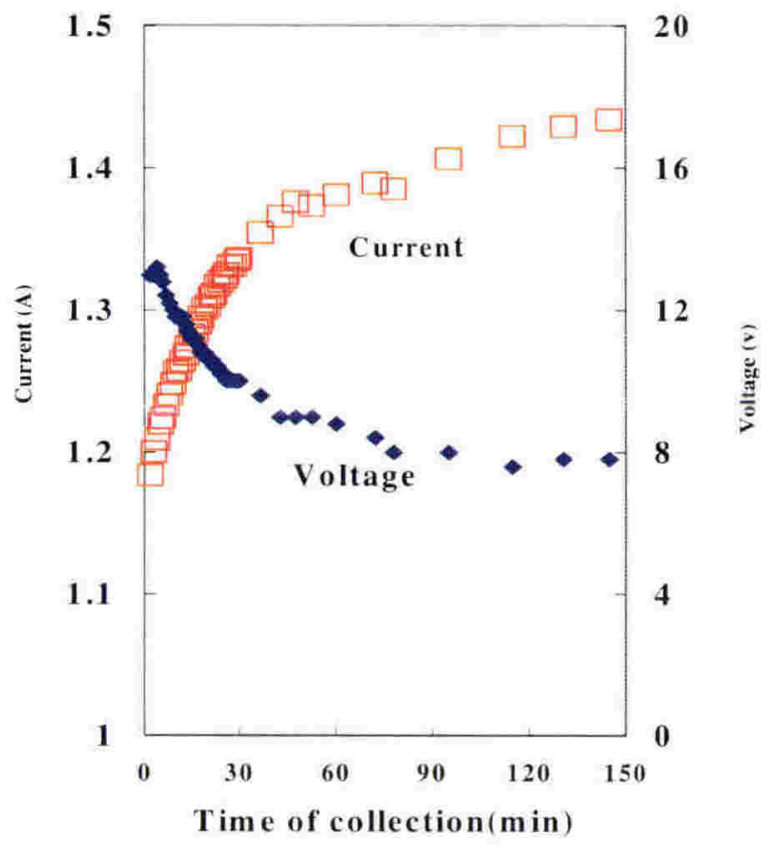

Figure 7-5 Plots of current and voltage across the array during a DEP selection. Hollow squares and solid diameters indicate current and voltage, respectively.

\subsubsection{Raman spectra}

Figure 7-6 (a) gives typical Raman spectra of original samples and those collected on the array. In this graph, there were a higher content of MWNTs in the samples collected on the array (the relative disorder intensity of CNTs dropped from 1.5 to 1 after the treatment, as seen from the Figure 7-6 (b)), compared with the original sample. This is evident of the fact that we were able to purify the as-grown CNT samples with our prototype DEP device.

Figure 7-6 (b) plots the ratio of the intensity of the D band over that of the G band for different samples. The data appearing at the initial point in the graph include dry samples, which were dropped on the array as well as the samples from the suspension (after being taken and dried) at the start of the collection. Raman spectroscopy investigations (The 
Chapter 7 Selective Purification of Multi-walled CNTs by Dielectrophoresis within a Large Array

numerous spectra are not be included here) show no noticeable difference between the two samples.
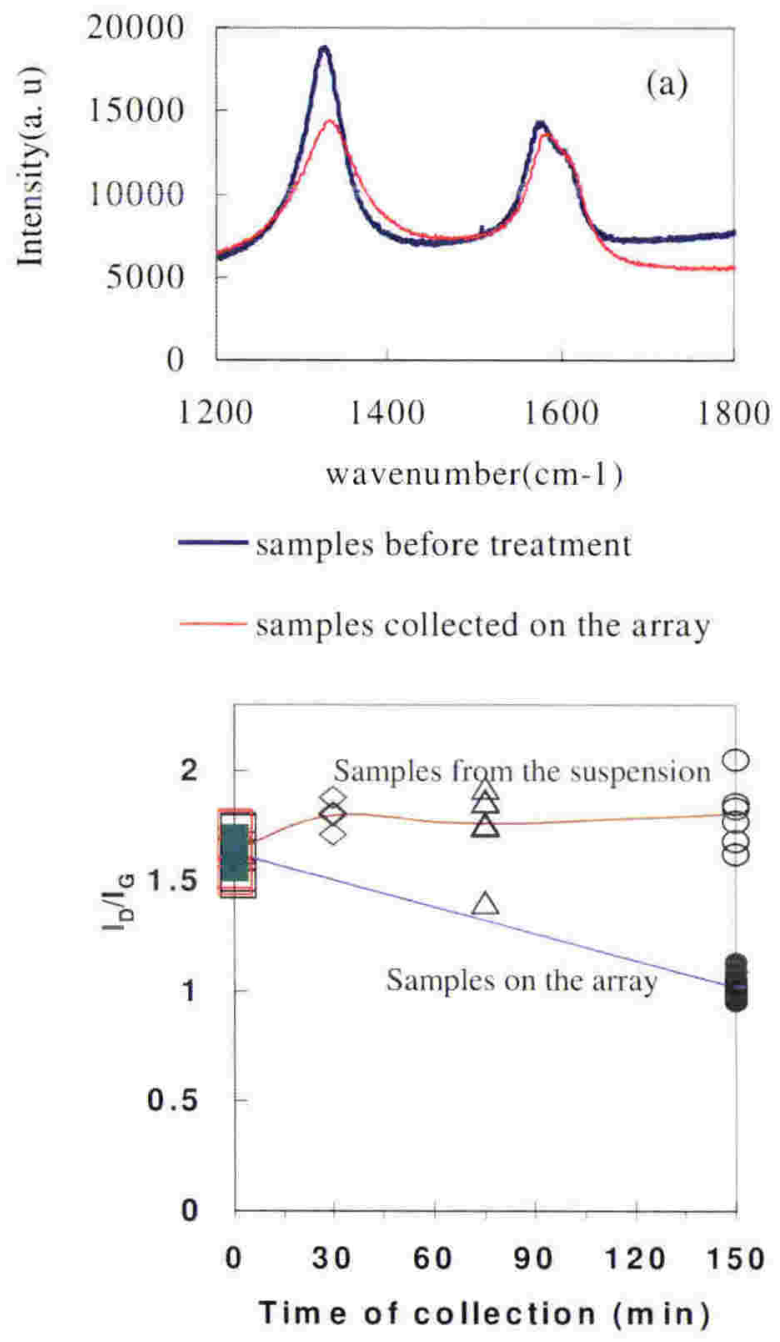

(b)

Figure 7-6 (a) A comparison between the typical Raman spectra of as-grown samples and samples collected on the array. (b) The relative intensity of the disorder band (D band) at $1335 \mathrm{~cm}^{-1}$ over the graphitic band ( $\mathrm{G}$ band) at $1585 \mathrm{~cm}^{-1}[5,15]$ versus time of collection. The two lines in the graph are plotted for guide of eye.

Raman spectra of MWNTs remaining in suspension at different instants of the separation process are given in Figure 7-7. These samples were taken at 30th, 75th and 150th minute of the separation process, respectively. 
Chapter 7 Selective Purification of Multi-walled CNTs by Dielectrophoresis within a Large Array

MWCNTs from the suspension

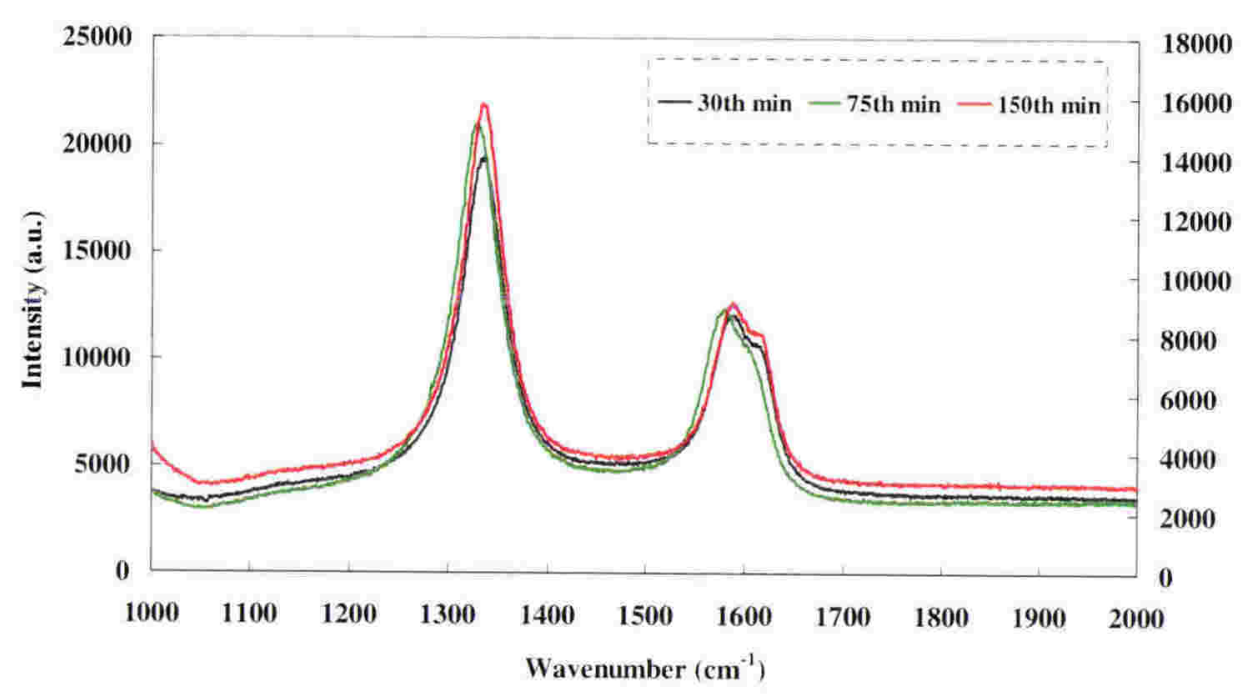

Figure 7-7 Typical Roman spectra of MWNTs sampled from the suspension at different instants of the separation process.

The plot in Figure 7-6 (b) and the Ramon spectra in Figure 7-7 show that the relative contents of disordered material against CATs in the residual suspension are larger than those of the samples before collection and the samples collected on the array. And that value keeps rising from the beginning of the selection process, which means more and

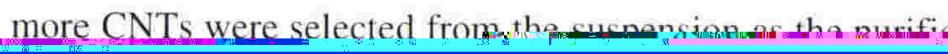




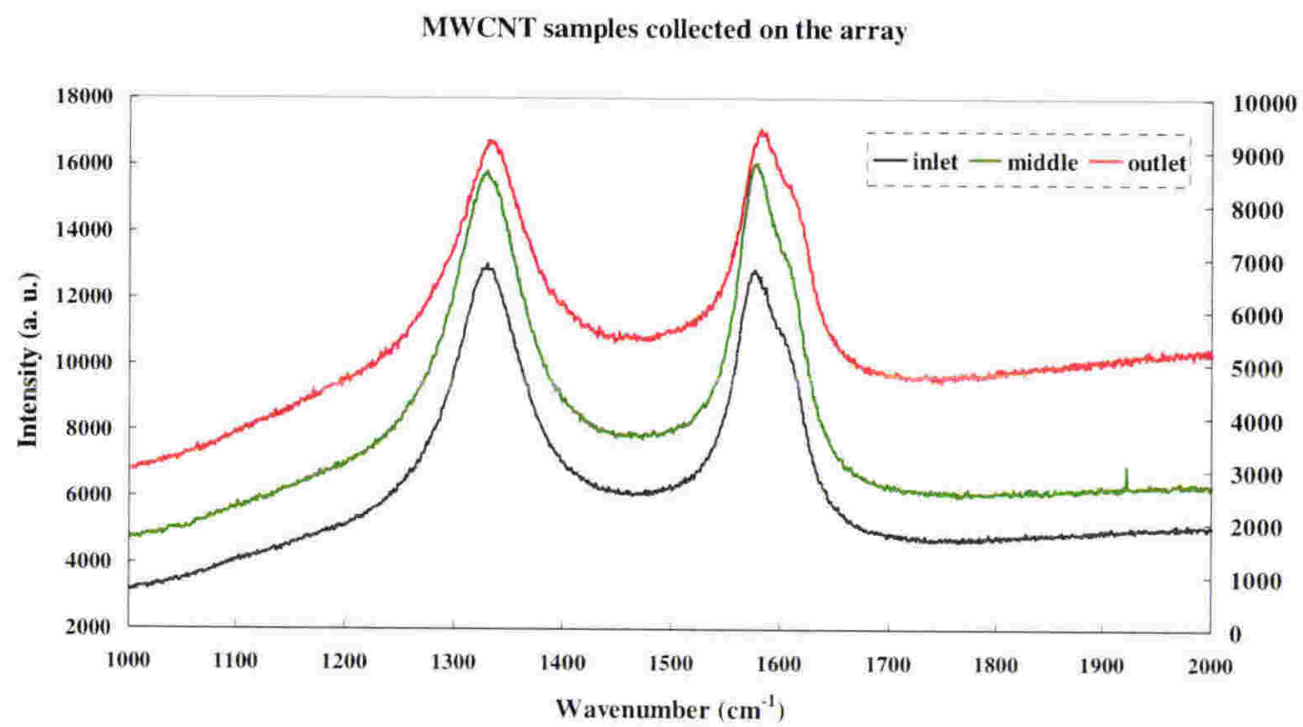

Figure 7-8 Raman Characterization of MWNT samples collected from different spots of the array after the DEP (the black, green and red plots represent the inlet, middle and outlet of the suspension flow, respectively.).

Observations under an optical microscope (Figure 7-3 (b) and (c)) show that the samples, which had been collected on the array, were not evenly distributed in different areas of the board. This can be attributed to the non-uniformities of the local electric field and the flowrate of CNTs dispersion in different spots inside the chamber. However, SEM images (a representative image is shown in Figure 7-4 (c)) and Raman characterization (in Figure 7-8) of the samples collected on the array demonstrated that the samples from different parts of the array, no matter whether they were collected on the electrodes or between them, were uniform in the purity of MWNTs.

Above all, the characterisation results from SEM and Raman demonstrated that this prototype device was successful in separating and purifying MWNTs from as-grown samples. The mechanism behind the device is that the large dielectrophoresis forces generated on the highly polarisable CNTs hold them onto the electrodes while impurities 
Chapter 7 Selective Purification of Multi-walled CNTs by Dielectrophoresis within a Large Array

were remained in the re-circulating suspensions. The selection process was operated at a lower frequency than the previous alignment process. The SEM and Raman characterization demonstrated that CNTs that were collected on electrodes were of higher purities than the original samples. This indicates that this device can be applied to largescale separation of MWNTs from as-grown impurities after optimisation.

\section{Reference:}

1. W. M. Arnold and R. Petherick (2002), Proc. of 9th Electronics New Zealand Conference: p. 1-5.

2. J. M. Bonard, T. Stora, J.-P. Salvetat, F. Marier, T. Stockli, C. Duschl, L. Forro, W. A. d. Heer, and A. Chatelain (1997), Advanced Materials, 9: p. 827-831.

3. X. Q. Chen, T. Saito, H. Yamada, and K. Matsushige (2001), Applied Physics Letters, 78: p. 3714-3716.

4. Y. K. Chen, M. L. H. Green, J. L. Griffin, J. Hammer, R. M. Lago, and S. K. Tsang (1996), Advanced Materials, 8: p. 1012-1015.

5. M. S. Dresselhaus, G. Dresselhaus, and P. C. Eklund (1996), in book. Science of Fullerences and Carbon Nanotubes San Diego: Elsevier Science

6. T. W. Ebbesen, P. M. Ajayan, H. Hiura, and K. Tanigaki (1994), Nature, 367: p. 519.

7. http://www.ahwahneetech.com/products/raw materials.htm.

8. S. Iijima (1991), Nature, 354: p. 56-58.

9. R. Kamalakaran, M. Terrones, T. Seeger, P. Kohler-Redich, M. Ruhle, Y. A. Kim, T. Hayashi, and Endo (2000), Applied Physics Letters, 77: p. 3385-3387. 

Array

10. C. J. Lee, D. W. Kim, T. J. Lee, Y. C. Choi, Y. S. Park, W. S. Kim, Y. H. Lee, W. B. Choi, N. S. Lee, J. M. Kim, Y. G. Choi, and S. C. Yu (1999), Applied Physics Letters, 75: p. 1721-1723.

11. X. Liu, J. L. Spencer, A. B. Kaiser, and W. M. Arnold (2002), Current Applied Physics, 4: p. 125-128.

12. X. Liu, J. L. Spencer, A. B. Kaiser, and W. M. Arnold (2002), Annual Report Conference on Electrical Insulation and Dielectric Phenomena, IEEE: p. 31-34.

13. H. A. Pohl (1978), in book. Dielectrophoresis: The Behavior of Neutral Matter in Nonuniform Electric Fields 1st ed Cambridge: Cambridge University Press

14. A. M. Rao, E. Richter, S. Bandow, B. Chase, P. C. Eklund, K. A. Williams, S. Fang, K. R. Subbaswamy, M. Menon, A. Thess, R. E. Smalley, G. Dresselhaus, and M. S. Dresselhaus (1997), Science, 275: p. 187-191.

15. R. Saito, G. Dresselhaus, and M. S. Dresselhaus (1998), in book. Physical properties of carbon nanotubes London: Imperial College Press

16. K. Tohji, T. Goto, H. Takahashi, Y. Shinoda, N. Shimizu, B. Jeyadevan, I. Matsuoka, Y. Saito, A. Kasuya, T. Ohsuna, K. Hiraga, and Y. Nishina (1996), Nature, 383: p. 679.

17. K. Yamamoto, S. Akita, and Y. Nakayama (1998), Journal of Physics D: Applied Physics, 31: p. L34-L36. 


\section{Chapter 8}

\section{Conclusions}

To take advantage of the many excellent properties and intriguing characteristics that carbon nanotubes exhibit because of their unique structures, it is necessary to develop techniques for the synthesis of CNTs, for removing impurities resulting from the synthesis process, and for aligning and manipulating CNTs so that they can be used in devices.

The work reported in this thesis contributes to these goals under two main headings: firstly, the synthesis of multi-walled carbon nanotubes by chemical vapour deposition was investigated in Part I; in Part II, the CNTs synthesized in Part I as well as CNTs from another source were used in an investigation of the application of dielectrophoresis for aligning and selecting of carbon nanotubes, and for constructing a device to separate CNTs from impurities.

\subsection{Synthesis of multi-walled carbon nanotubes by chemical vapour deposition}

The first part of this thesis describes how MWNTs were grown on a $\mathrm{SiO}_{2}$ surface by chemical vapour deposition (CVD) from three different precursors: $\mathrm{Fe}\left(\mathrm{NO}_{3}\right)_{3}$, ferrocene and FePC. The investigation of the multi-walled CNT structures by HRTEM confirmed that carbon nanotubes synthesized in our experiments consisted of tubular graphitic 
lattices uniformly separated by $0.34 \mathrm{~nm}$, which is a key characteristic of MWNTs. As characterised by SEM and TEM, there is no alignment shown in the morphology of CNTs from $\mathrm{Fe}\left(\mathrm{NO}_{3}\right)_{3}$ catalysts and these CNTs contain many more defects and curvatures than those from the other two precursors, which did lead to alignment.

Vertically aligned carbon nanotubes were grown on silica substrates from the two latter precursors inside a dual furnace system. The vertical alignment of CNTs is attributed to the "overcrowding effect" which means that nanotubes are grown from a high density of catalysts and the inter-nanotube van der Waals forces attach the nanotubular walls together enabling carbon radicals to grow vertically into aligned carbon nanotube films. There are some common characteristics among the vertically aligned carbon nanotubes: these nanotubes are MWNTs with lengths of 10 to $20 \mu \mathrm{m}$ and diameters of less than 100 nm. However, differences were observed between the two kinds of vertically aligned nanotubes from different precursors from various characterisation measures such as TEM, SEM, EDX, TGA and Raman.

The TEM microscopy showed that catalyst-derived impurities were observed in bases and tips of CNTs from ferrocene; on the other hand, catalysts are only observed from the roots of CNTs from FePC. As observed by SEM, the latter tubes have straighter and more perfect individual structure and fewer inter-tube tangles, and these tubes are more uniform than those from ferrocene. Characterized by TGA, the median burning temperature of FePC CNTs in airflow is $46{ }^{\circ} \mathrm{C}$ higher than that of ferrocene CNTs. This observation is consistent with those from SEM, TEM, Raman and EDX. These differences in morphology and stability of CNTs are attributed to different growth mechanisms and precursors. It has been proposed in this thesis that the ferrocene tubes 
follow tip and base growth mechanism and FePC tubes are from the base growth mechanism. Also we need to consider the differences in physical properties and compositions of the two precursors. Ferrocene has a larger temperature range of evaporation and is more chemically volatile than FePC. And compared with FePC (molar ratio, $\mathrm{Fe} / \mathrm{C}: 1 / 10)$, the ratio of $\mathrm{Fe}$ content in $\mathrm{FePC}(\mathrm{Fe} / \mathrm{C}: 1 / 32)$ is much less. We infer that the greater volatility and greater $\mathrm{Fe} / \mathrm{C}$ ratio of ferrocene may be related to the less uniformly sized catalyst particles on growth substrates and therefore the poorer quality of CNTs.

In addition, a pre-templated $\mathrm{Si}_{3} \mathrm{~N}_{4}$ substrate with $\mathrm{Al}$ and $\mathrm{Au}$ patterns, which had been fabricated by a lithographic method, was used to replace the silica substrate in a CVD process. It was found that CNTs did not deposit on the Au surface. Selective and patterned growth of CNTs was only obtained on the $\mathrm{Si}_{3} \mathrm{~N}_{4}$ substrate and the places where $\mathrm{Al}$ evaporated and left $\mathrm{Si}_{3} \mathrm{~N}_{4}$ substrate exposed. The selective growth of CNTs yielded a microstructure which was based on patterned CNTs, $\mathrm{Au}$ and $\mathrm{Si}_{3} \mathrm{~N}_{4}$ and provided a viable route to combine silicon-based devices with carbon nanotube-based devices for further MEMS applications. With a more advanced pre-pattern lithographic technique with a higher resolution of metal layout, this strategy can be even extended to NEMS applications.

\subsection{Application of dielectrophoresis for aligning and selecting of carbon nanotubes}

Since the discovery of CNTs, post-growth treatments of CNTs have been of significant interest. However, manipulation and selection of CNTs still remain technical challenges because of their tiny dimensions. As pseudo-one dimensional conductors, CNTs can be 
electrically polarised in an AC electric field. In the second part of this thesis, dielectrophoresis (DEP) was employed as an approach for these two purposes.

In our experiments, MWNT suspensions were dropped on interdigitated electrodes (IDEs), across which AC voltages were applied. Alignment of nanotubes from suspensions along their axis was obtained between the opposite electrodes when an AC voltage at a frequency more than $1.1 \mathrm{MHz}$, which is the crossover frequency for our MWNTs, was applied. Observed by SEM, the alignment was established by nanotube bundles joining head to head with each other between the electrodes. The real-time changes of $\mathrm{AC}$ conductance and capacitance between electrodes during the alignment process were recorded and found to be reflections of the bridging of CNTs between electrodes. Different behaviours in alignments and the AC conductance and capacitance changes occurred on electric field-collected CNTs from FePC and ferrocene.

Accumulations of CNTs from FePC-derived catalysts caused progressive increases in both capacitance components and $\mathrm{AC}$ conductance, which indicates no apparent ohmic contact with the electrodes. The dependence of the capacitance on the reciprocal of electric field frequency has been observed and is consistent with an interfacial-type of polarisation mechanism in dielectrophoresis; in another word, the electric current paths from these nanotubes to the electrodes were through at least a thin layer of liquid medium, rather than by direct contact between CNTs and electrodes.

By contrast, MWNTs formed on ferrocene-derived catalysts are capable of forming "bridging" paths, which were highly conductive. The accumulation of this kind of MWNT was accompanied by rapid increase of the AC conductance and decrease of 


\section{Chapter 8 Conclusions}

capacitance between the electrodes. It was proposed in this thesis that the conductive paths resulted from the direct contact between CNTs and electrodes.

This difference could arise from an intrinsic conductivity difference due to the greater iron content in MWNTs from ferrocene-derived catalysts, as well as from larger tube thickness and greater foldings.

The response of the alignment of FePC-derived CNTs to the on/off switches of electric field was also investigated in our experiment. The alignment of CNTs as well as the admittances between the electrodes shows changes corresponding to the on/offs of the electric field. This phenomenon indicates that the bridging of CNTs between electrodes is responsible for the increases of $\mathrm{AC}$ conductance and capacitance.

In addition, the effects of solvents on alignment of CNTs were demonstrated and it was shown that distilled water is a better candidate for CNTs' dielectrophoresis than ethanol and methanol because of its larger relative permittivity.

\subsection{A prototype device for separating MWNTs and impurities}

Based on the results in Part II, a prototype device, which consisted of a $130 \mathrm{~mm} \times 134$ mm IDE array, a chamber and a pump which facilitates the flow of a re-circulating CNT suspension, was built to separate MWNTs from as-grown impurities by combining dielectrophoretic force and flowing liquid. The mechanism behind the device is that the large dielectrophoresis forces generated on the highly polarisable CNTs hold them onto the electrodes while impurities remained in the re-circulating suspensions. The selection process was operated at a lower frequency than the previous alignment process. The SEM and Raman characterization demonstrated that CNTs that were collected on electrodes 
were of higher purities than the original samples. This indicates that this device can be applied to large-scale separation of MWNTs from as-grown impurities after optimisation.

Alignment can reduce the load of CNTs required in conductive CNTs/polymeric composites. DEP is a simple after-synthesis process to achieve alignment of CNTs. Based on the results reported in this thesis, the DEP technique can be extended to fabricate nanometre- and submicron-scale structures which are based on CNTs or CNTs/polymeric matrix.

\subsection{Electro-spinning of Poly Ethylene Oxide (PEO) fibres}

An approach to produce composites in the form of fibre is described in the Appendix. The early work on this thesis demonstrated the production of PEO fibres, both insulating and conductive (with carbon black added as conducting agent). 


\section{Appendix}

\section{Electro-spinning of PEO Fibres}

When an electric field at a very high voltage is applied between a drop of polymeric solution and a target, polymeric fibres with less than 1 micron diameter can be obtained at the target. This technique is named "electro-spinning" due to its common characteristic with fibre-spun process, which has a long history in fibre industry. Electro-spinning is an easy and simple approach to produce polymer fibres with small diameters. A typical setup for electro-spinning is given in Figure A-1. Electro-spinning of polyethyleneoxide (PEO) was investigated as part of the $\mathrm{PhD}$ experimental work. Also, a rotating drum was used to introduce alignment on electro-spun PEO fibres.

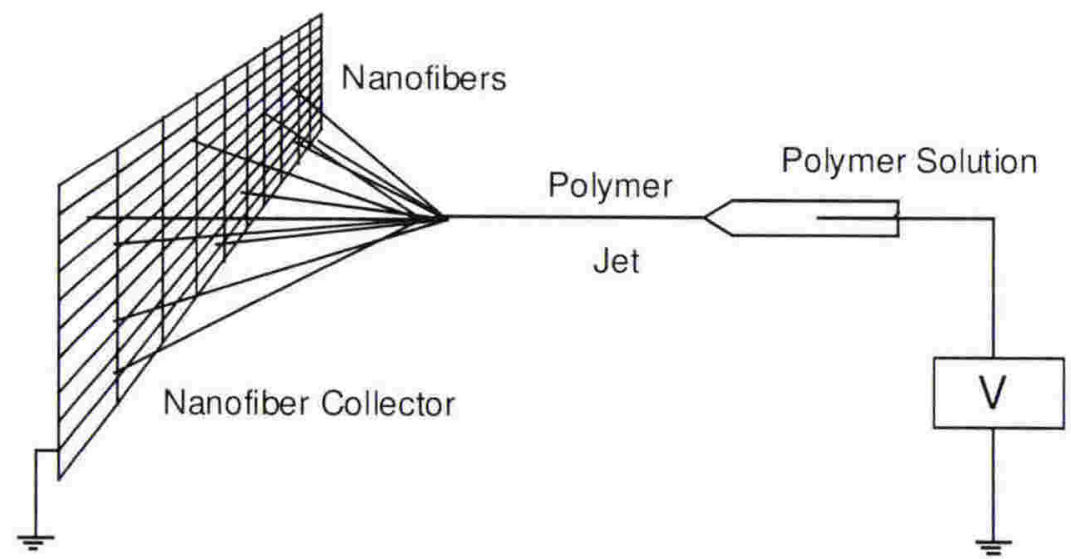

Figure A-1 A typical set-up of an electro-spinning process.

In this appendix, it is demonstrated that PEO fibres were electro-spun at $30 \mathrm{kV}$ onto a rotating drum with high RPMs. As depicted in Figure A-2, the drum was positioned 
around a charged $\mathrm{Al}$ plate, to introduce the alignment produced by rotation. Such a design is commercially feasible on an industrial scale.

\section{EXPERIMENT}

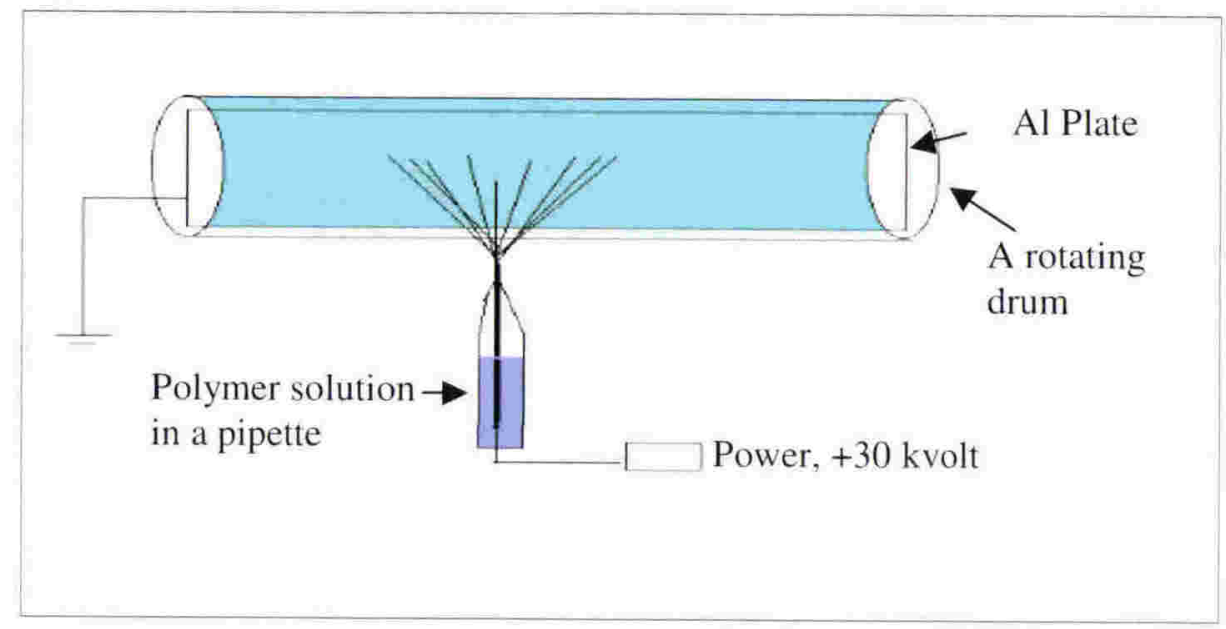

Figure A-2 Depict of an electro-spinning apparatus, with a rotating drum which introduces alignment upon PEO fibres.

Polyethyleneoxide (PEO), purchased from Aldrich, with an average molecular weight of 300,000 , was dissolved in distilled water, assisted by stirring with a concentration of $5 \mathrm{w} / \mathrm{w} \%$. Then $1.0 \mathrm{ml}$ PEO solution was placed in a pipette with a copper wire, which was connected to a power supply of +30 kilovolts. An aluminium plate was connected to the earth. The distance between the copper wire and the Al plate was kept at $28 \mathrm{~cm}$.

\section{(1) PEO fibres collected on a rotating drum}

A piece of A4 transparent plastic overhead slide was folded into a hollow drum, which was then rotated by a motor, with a maximum speed of 700 RPM. The rotating drum was placed around the Al plate, which was installed as the cathode, as shown in Fig. A3 (b). 
Appendix Electro-spinning of PEO Fibres

PEO fibres were collected on the surface of the drum cylinder. The rotating rate of the drum was kept at 700 RPM.

\section{(2) Preparation of PEO fibres containing carbon black}

PEO powders were stirred and added to a suspension of carbon black in distilled water, and then the suspension was ultrasonicated for 30 minutes in water bath. Small amount of the resulting suspension was placed in a glass pipette and fibres were deposited onto the Al plate by electro-spinning.

For mixtures of PEO and carbon black in suspensions, PEO versus carbon black at different ratio of $4: 1,2: 1$ and 1:2 were employed. The electrospinning process of PEO fibres containing carbon black is as the same described in the last section.

\section{(3) SEM microscopy}

The samples collected on drums were collected, and investigated by SEM microscopy (a Philips EM 505 Scanning Electron Microscope), which was operated at $5.0 \mathrm{kV}$ after being coated by $4 \mathrm{~nm} \mathrm{Au}$.

\section{RESULTS AND DISCUSSIONS}

After the power was switched on, the polymeric solution in the pipette was charged and it can be viewed that, jets of polymeric solution were sprayed from the pipette and dropped on the rotating drum. The forces, which confined the movement of the jets, can be attributed to the electrostatic forces generated by the electric field between the copper wire and the Al plate. Each electro-spinning process lasts for 30 seconds.

\section{(1) PEO fibres collected on a rotating drum}


Appendix Electro-spinning of PEO Fibres

After electro-spinning, a thin mat of PEO fibres, with diameters from 100 to $200 \mathrm{~nm}$ was deposited on the outer surface of the rolling drum. Comparison was made between fibres collected on the drum with a stationary state and a rolling state at 700 RPM.
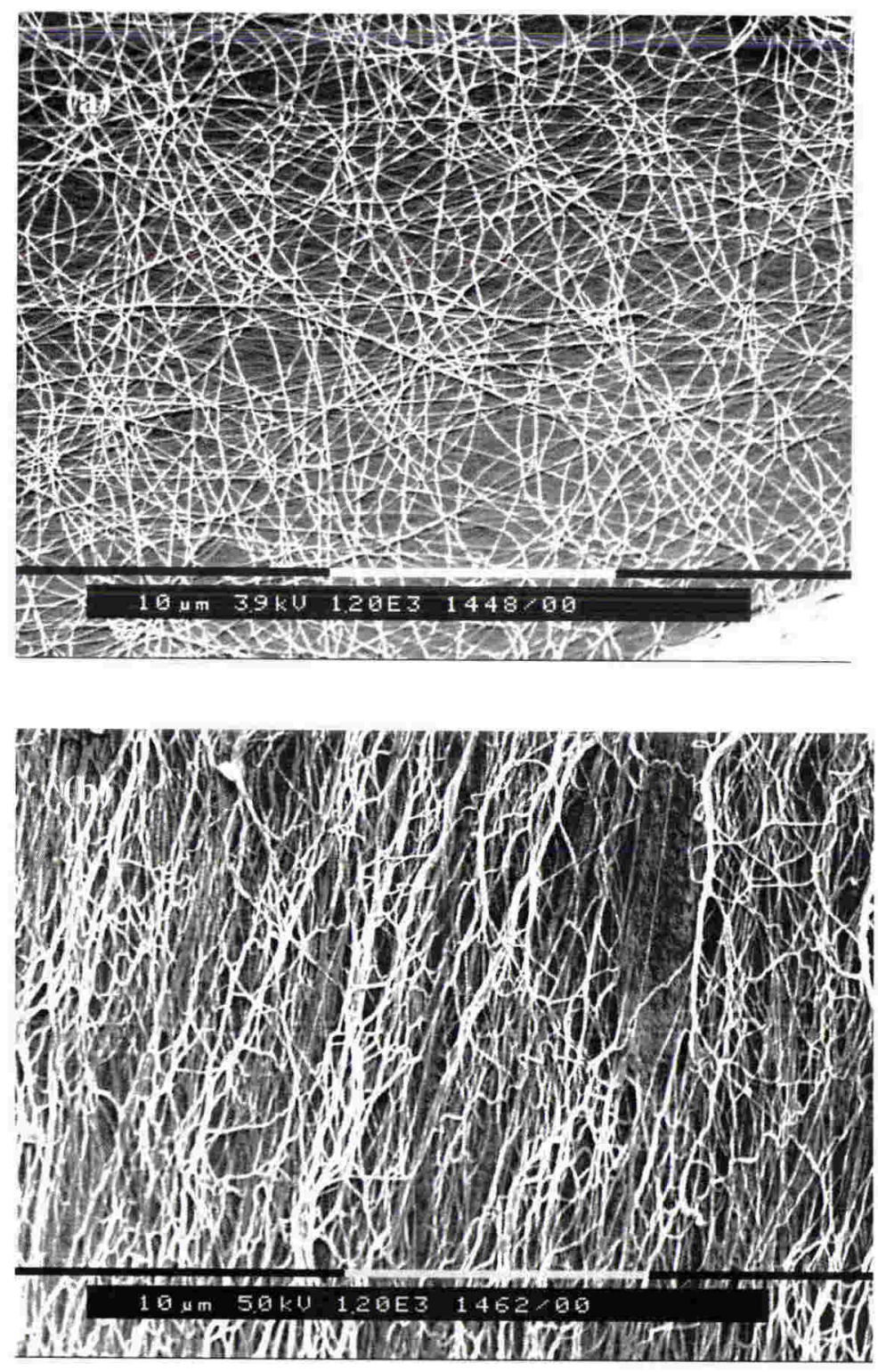

Figure A-3 Electro-spun PEO fibres collected on the drum. (a) When the drum did not rotate; (b) when the drum rotates at $700 \mathrm{rpm}$. 


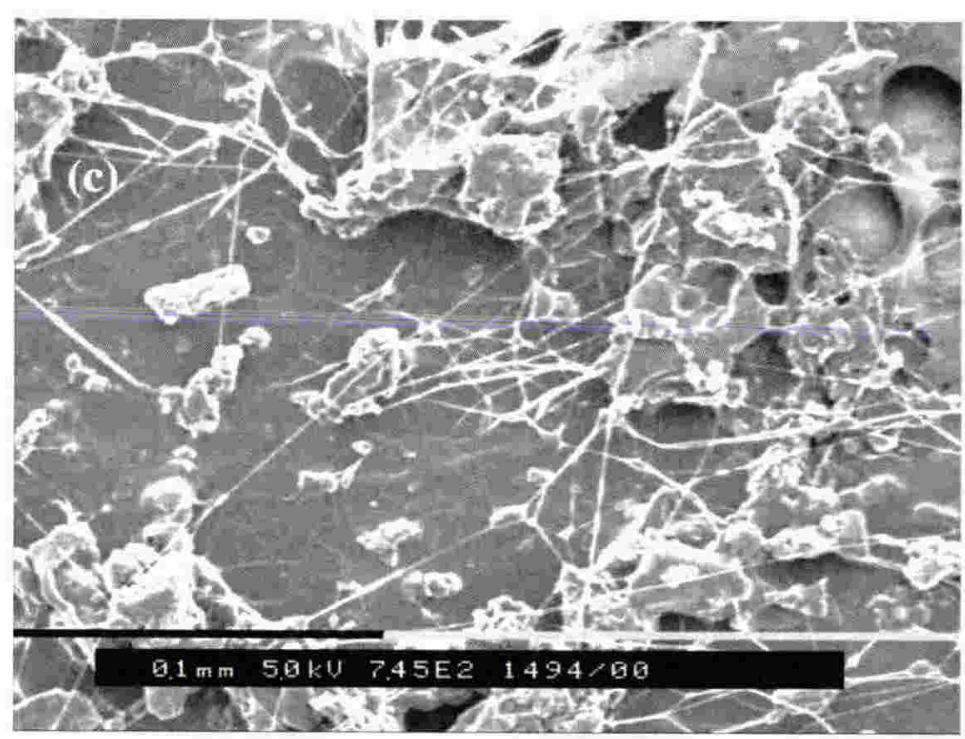

Figure A-4 An SEM image of fibres of PEO /carbon black at ratios of (a) 4:1; (b) $2: 1$; (c) $1: 2$.

(2) Preparation of PEO fibres containing carbon black.

Fibres were electro-spun from different ratios of PEO and carbon black. The SEM images in Figure A-4 show the different morphologies obtained for the different ratios.

It was demonstrated in Figure A-4 that, more blocks and less and wider fibres appear as the relative content of carbon black in the mixture increases. 